\title{
Thermal Decomposition of Nitrated Tributyl Phosphate
}

by

D. F. Paddleford

Westinghouse Savannah River Company

Savannah River Site

Aiken, South Carolina 29808

Y. Hou

Georgia Institute of Technology

GA USA

E. $K$ Barefield

Georgia Institute of Technology

GA USA

D. W. Tedder

Georgia Institute of Technology

GA USA

S. I. Abdel-Khalik

Georgia Institute of Technology

GA USA

DOE Contract No. DE-AC $19-89$ SR18035

This paper was prepared if connection with work done under the above contract number with the U.S.

Department of Energy. $\forall y$ acceptance of this paper, the publisher and/or recipient acknowledges the U.S.

Government's right to retain a nonexclusive, royalty-free license in and to any copyright covering this paper, along with the right tor reproduce and to authorize others to reproduce all or part of the copyrighted paper. 


\title{
THERMAL DECOMPOSITION OF NITRATED TRIBUTYL PHOSPHATE
}

\author{
Final Report \\ RECEIVED \\ Submitted to \\ MAY 081996 \\ Westinghouse Savannah River Company \\ OSTI \\ (Task Order \#94054 of ERDA Subcontract \#AA46420T)
}

\section{By}

Y. Hou ${ }^{1}$, E. K. Barefield ${ }^{2}$, D. W. Tedder ${ }^{3}$, and S. I. Abdel-Khalik ${ }^{1}$

Georgia Institute of Technology

January, 1995

\footnotetext{
${ }^{1}$ School of Mechanical Engineering

${ }^{2}$ School of Chemistry

${ }^{3}$ School of Chemical Engineering
}

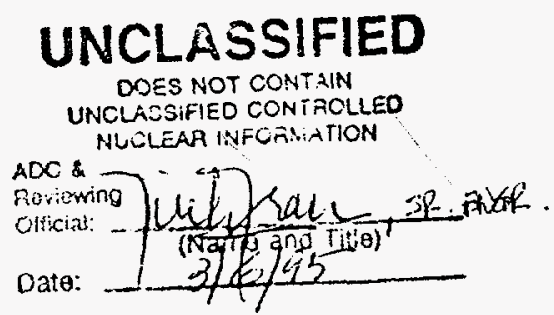




\section{DISCLAIMER}

This report was prepared by Westinghouse Savannah River Company (WSRC) for the United States Department of Energy under Contract No. DE-AC0989SR 18035 and is an account of work performed under that contract. Reference herein to any specific commercial product, process, or service by trademark, name, manufacturer or otherwise does not necessarily constitute or imply endorsement, recommendation, or favoring of same by WSRC or by the United States Government or any agency thereof. The views and opinions of the authors fexpressed herein do not necessarily state or reflect those of the United States Government or any agency thereof. 


\section{DISCLAIMER}

Portions of this document may be illegible in electronic image products. Images are produced from the best available original document. 
Preface

The enclosed final report, GTRSR-023, "Thermal Decomposition of Nitrated Tributal Phosphate" summarizes experiments conducted by Georgia Institute of Technology for WSRC during the period July 1994 through January 1995. The experiments were in support of the TBP/nitric acid operations safety evaluation. Guidance and planning for the experiments were provided by D. F. Paddleford, M. L. Hyder, and $M$. C. Thompson of WSRC technical staff. Also attached are the Georgia Tech Technical Proposal (Attachment \#1) and Monthly Progress Reports (Attachment \#2) for this project. 


\title{
THERMAL DECOMPOSITION OF NITRATED TRIBUTYL PHOSPHATE
}

\author{
Final Report \\ Submitted to
}

Westinghouse Savannah River Company

(Task Order \#94054 of ERDA Subcontract \#AA46420T)

By

Y. Hou', E. K. Barefield ${ }^{2}$, D. W. Tedder ${ }^{3}$, and S. I. Abdel-Khalik ${ }^{1}$

Georgia Institute of Technology

January, 1995

! School of Mechanical Engineering

UNCLASSIFIED

DOES NOT CONTAN

${ }^{2}$ School of Chemistry

${ }^{3}$ School of Chemical Engineering

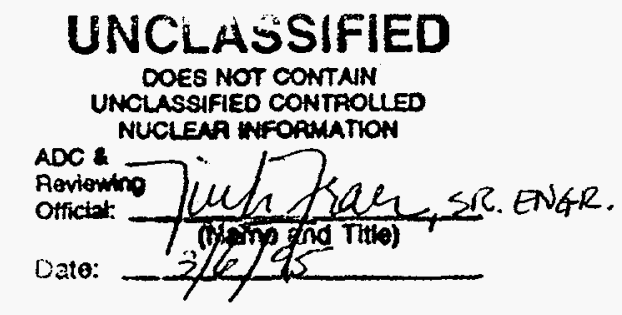




\section{CONTENTS}

Abstract

List of Abbreviations and Acronyms

List of Tables

List of Figures

$\begin{array}{ll}\text { I. INTRODUCTION } & 1\end{array}$

II. EXPERIMENTAL PROCEDURES $\quad 2$

II.1. Sample and Standards Preparation $\quad 2$

II.2. Procedure for Constant Pressure Decomposition $\quad 2$

a. Reflux Conditions 2

b. Distillation Conditions 3

II.3. Procedure for Constant Volume Decompositions 4

II.4. Reactant and Product Analyses

III. RESULTS $\quad 5$

III.1. Effect of Temperature $\quad 5$

III.2. Effect of Nitric Acid Concentration $\quad 6$

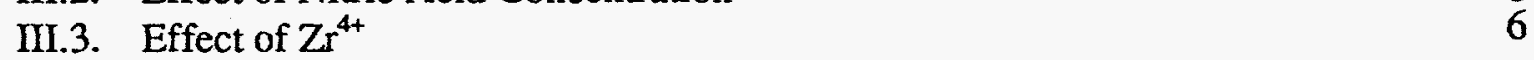

III.4. Effect of Reduced Water Content $\quad 7$

III.5. Possible Origin of Products $\quad 7$

IV. CONCLUSIONS AND RECOMMENDATIONS 9

V. REFERENCES

\section{APPENDICES}
A. Analytical Data
A- 1
B. Effect of Acid Concentration (SP, CP, D, $150^{\circ} \mathrm{C}$ )
A-36
C. Effect of Acid Concentration (SP, CV, $150^{\circ} \mathrm{C}$ )
A -40
D. Effect of Acid Concentration (TP, CP, D, $150^{\circ} \mathrm{C}$ )
A-44
E. Effect of Acid Concentration (TP, CV, $150^{\circ} \mathrm{C}$ )
A-48
F. Effect of Reduced Water Content
A-52
G. Effect of Temperature (SP, CP, 6M, D)
A-57
H. Effect of Temperature (SP, CP, 6M, R)
A-62
I. Effect of Temperature (SP, CV, 6M)
A-66
J. Effect of Temperature (TP, CP, 6M, D)
A-70
K. Effect of Temperature (TP, CP, 6M, R)
A-74
L. Effect of Temperature (TP, CV, 6M)
A-78
M. Effect of $\mathrm{Zr}^{4+}\left(\mathrm{SP}, \mathrm{CP}, 6 \mathrm{M}, \mathrm{D}, 150^{\circ} \mathrm{C}\right)$
A-82
N. Effect of $\mathrm{Zr}^{4+}\left(\mathrm{SP}, \mathrm{CV}, 6 \mathrm{M}, 150^{\circ} \mathrm{C}\right)$
A-87
O. Comparison Between Distillation and Reflux Conditions 


\begin{abstract}
Tri-n-butyl phosphate (TBP), nitric acid and water mixtures are subject to thermal decomposition. The gaseous and liquid decomposition product yields are highly path dependent. Higher temperatures accelerate decomposition rates, but may result in lower extent of reaction than romprarable low temperature cases. Actual extent of reaction, and gaseous by-products generation, are affected by the presence of $\mathrm{Zr}(\mathrm{IV})$, condensate reflux back into the reaction vessel, the water $/ \mathrm{HNO}_{3}$ and the $\mathrm{HNO}_{3}$ /TBP molar ratios, and whether the decomposition occurs at isobaric or constant volume conditions. Higher gaseous production results from condensate reflux, lower $\mathrm{H}_{2} \mathrm{O} / \mathrm{HNO}_{3}$ ratios, and with excess $\mathrm{HNO}_{3}$ and water present as a second liquid phase. Principal gaseous products include $\mathrm{N}_{2}, \mathrm{CO}, \mathrm{CO}_{2}, \mathrm{NO}$ and $\mathrm{N}_{2} \mathrm{O}$. Measurable concentrations of $\mathrm{NO}_{2}$ were not observed, although gas coloration indicative of $\mathrm{NO}_{2}$ were observed during the beginning of decomposition measurements. Principal liquid products are dibutyl phosphoric acid and butyl nitrate. Air sparging of TBP solutions (e.g., thru transfers using air lift pumps) may increase the hazards of TBP decomposition reactions by lowering the $\mathrm{H}_{2} \mathrm{O} / \mathrm{HNO}_{3}$ molar ratios. Runaway reactions were not observed under the conditions of this study. Possible reaction mechanisms and pathways are discussed.
\end{abstract}




\section{List of Abbreviations and Acronyms}

$\begin{array}{ll}\text { CP } & \text { Constant Pressure } \\ \text { GV } & \text { Constant Volume } \\ \text { DR: } & \text { Dibuty! Phosphate } \\ \text { D } & \text { Disiillation } \\ \text { GC } & \text { Gas Chromatography } \\ \text { MBP } & \text { Monobutyl Phosphate } \\ \text { NMR } & \text { Nuclear Magnetic Resonance } \\ \text { R } & \text { Reflux } \\ \text { SP } & \text { Single Phase } \\ \text { TP } & \text { Two Phase } \\ \text { TBP } & \text { Tributyl Phosphate }\end{array}$


WSRC-RP-95-259

\section{List of Tables}

Table $1 \quad$ List of Completed Experiments 
WSRC-RP-95-259

\section{List of Figures}

Figure 1 Apparatus for Gas Volume Measurement at Constant Pressure Condition

Figure 2 Apparatus for Gas Pressure Measurement at Constant Volume Condition 
WSRC-RP-95-259

\section{INTRODUCTION}

Contact between tributyl phosphate (TBP) and aqueous solutions of nitric acid and/or heavy metal nitrate salts at elevated temperatures can lead to exothermic reactions of explosive violence. Even though such nperations have been routinely performed safely for decades as an intrinsic part of the - urex separation processes, several so-called "red oil" explosions are known to have occurred in tine United States, Canada, and the former Soviet Union. ${ }^{1,2}$ The most recent red oil explosion occurred at the Tomsk-7 separations facility in Siberia, in April, 1993. That explosion destroyed part of the unreinforced masonry walls of the canyon-type building in which the process was housed, and allowed the release of a significant quantity of radioactive material.

Numerous studies were performed following the first (1953) explosions at both Hanford and Savannah River, and the subsequent (1975) explosion at Savannah River. These studies were aimed at establishing safe conditions for the handling of nitric acid and TBP during Purex and related chemical processing. ${ }^{1,2}$ The recent Tomsk-7 explosion has renewed concerns about Purex process safety and the potential impact of red oil explosions. The concerns have been motivated by the assertion that unlike all other prior incidents involving TBP-nitrate reactions, the Tomsk-7 explosion ostensibly did not involve an external heat source. ${ }^{1}$ These concerns have raised questions about the effects of a similar explosion occurring in the SRS separations processes. To this end, numerous investigations have been recently conducted. A review of prior and ongoing research in this area is given by Hyder. ${ }^{1}$

Recently, Fauske and Associates performed adiabatic calorimetry tests to determine the venting requirements for safe operation of nitric acid/TBP processes. ${ }^{3}$ The data suggest that vent areas per unit mass greater than $\sim 1 \times 10^{-2} \mathrm{~mm}^{2} / \mathrm{g}$ would prevent overpressurization in such systems. Differential scanning calorimetry was used to determine the energy release in open (vented) nitric acid/TBP reactions; ${ }^{4,5}$ values ranging from 7.2 to $140 \mathrm{cal} / \mathrm{g}$ were reported. In addition to detailed laboratory studies, scoping calculations were performed to estimate the order-of-magnitude consequences of a red-oil explosion in the SRS F-canyon. ${ }^{6}$ In order to assess the severity of such explosions, it is necessary to estimate the yield of volatile flammable gases that may be produced during such reaction. To this end, this investigation was undertaken to characterize the products of decomposition reactions involving nitrated tributyl phosphate. The effort has been focused on analytical techniques to accurately and reproducibly provide material balance information describing the decomposition products in both the gaseous and liquid phases. A total of thirty three experiments have been conducted at various temperatures, and acid concentrations for both single (organic) phase and two-phase systems in the presence and absence of $\mathrm{Zr}^{4+}$ (saturated). Experiments were conducted under both constant pressure (ambient) and constant volume conditions. 


\section{EXPERIMENTAL PROCEDURES}

\section{II.1. Sample and standards preparation}

Samples were prepared using the highest purity reagents that were available. Tri-n-butyl phosphate (TBP), 99\%, was obtained from Aldrich Chencical Co. and used as received. Reagent grade nitric acid was diluted with distilled water as required. All solutions were purged with helium and stored in sealed vessels under an helium atmosphere.

Fisher standard $1 \mathrm{M}$ and $0.2 \mathrm{M} \mathrm{NaOH}$ were used to determine acid concentrations using bromthymol blue as the indicator. Nominal $9 \mathrm{M} \mathrm{HNO}_{3}$ was prepared using concentrated reagent grade acid diluted with distilled water. Titration indicated the actual acid concentration was $8.9 \mathrm{M}$. This solution was used for all equilibrations with TBP to generate $3 \mathrm{M}$ TBP solutions that are described in this report. Reagent grade nitric acid was used for equilibrations to generate the $6 \mathrm{M}$ equilibrated TBP solutions.

Concentrations of water in equilibrated samples and in products and residues were determined by Karl Fisher titration.

Equilibrations with TBP and $9 \mathrm{M}$ acid were done by stirring equal volumes of each component. Equilibrations of TBP and concentrated acid $(\sim 16 \mathrm{M})$ were done using one part of TBP and three parts of the concentrated acid. The two phase system was purged with helium for 30 minutes, sealed and allowed to equilibrate at room temperature with gentle stirring. Titrations of aliquots taken from the aqueous phase as a function of time indicated that equilibrium was achieved after 24 hours of mixing. Equilibrations with the $9 \mathrm{M}$ acid gave acid concentrations of $3.00 \mathrm{M}$ in the organic phase and $6.30 \mathrm{M}$ acid in the aqueous phase. Equilibrations with concentrated acid gave acid concentrations of $5.75 \mathrm{M}$ in the organic phase and $\sim 15 \mathrm{M}$ acid in the aqueous phase. Portions of all equilibrated solutions have been stored at room temperature and at $50^{\circ} \mathrm{C}$ under helium since their preparation to determine the effects of aging.

For experiments that involved the addition of $\mathrm{Zr}(\mathrm{IV})$ one volume of pure TBP and three volumes of $\mathrm{HNO}_{3}$ ( either concentrated or 9M) that was saturated in $\mathrm{ZrO}\left(\mathrm{NO}_{3}\right)_{2}$ (about $0.1 \mathrm{M}$ ) were combined. Helium was used to purge the mixture for 30 minutes before it was sealed and stirred for 24 hours. After this time acid concentration in each phase was similar to that in the absence of the Zr(IV) salt.

\section{II.2. Procedure for constant pressure decompositions}

\section{a. Reflux conditions}

Decomposition reactions of equilibrated TBP/nitric acid samples were conducted in the apparatus shown in Figure 1, which was modified to incorporate sampling valves for obtaining gas samples for mass spectroscopy and infrared spectroscopy. This apparatus allows for volumetric determination of gas production up to $600 \mathrm{~mL}$. In runs where the volatiles were collected, the condenser was repositioned to allow for distillation rather than reflux and a receiver placed between the condenser and gas 
measurement system. Runs done under reflux conditions had sample temperatures that were about $15^{\circ} \mathrm{C}$ less than the bath temperature. The reaction vessel was a 50 $\mathrm{mL}$ three-necked flask, which was immersed in an oil bath up to the neck of the flask. A thermocouple was inserted into the sample to monitor its temperature.

Experiments were conducted by placing a known volume of cuiuilitraterl TBP mixture into a tared flask and the mass of the sample determined by difference. The flask was fitted to the condenser and the system evacuated and back-filled with helium several times. A preheated oil bath was raised such that the flask was immersed to the neck. The system was vented to the atmosphere for a short time $(<2 \mathrm{~min})$ to maintain atmospheric pressure while the temperature equilibrated. At this point the time was recorded and periodic measurements of the volume of gas produced were made. At the completion of a run, gas samples were removed for mass and infrared spectroscopic analysis. The oil bath was lowered and, after cooling to room temperature, the flask was removed, sealed (with tared stopper), and cleaned with solvent. At this point the mass of the residue was determined and then samples were removed for NMR analysis and for titration of acid and water content.

For runs through \#146 gas samples were taken immediately at the end of an experiment. A $30 \mathrm{~mL}$ sample container was evacuated and connected to the gas collection apparatus. The connecting stopcock was opened to allow the gas to expand into the evacuated container. The sample container was evacuated and filled three more times; in each case the pressure in the system was adjusted to atmospheric prior to evacuation by adjustment of the liquid reservoir. Gas sampled during the last filling was used for the GC analysis.

For runs after \#146 gas samples are again taken immediately after the cessation of gas evolution. However, a $200 \mathrm{~mL}$ sample container was substituted for the $30 \mathrm{~mL}$ container referred to above. The product gases are allowed to expand into the evacuated container. If less than $200 \mathrm{~mL}$ of gas was produced in a reaction it was necessary to add helium to bring the pressure in the system to atmospheric before detaching the container for GC analysis.

\section{b. Distillation Conditions}

The apparatus shown in Figure 1 was adapted for distillation by fitting a distillation head between the reaction flask and condenser and a receiver head and collection flask between the condenser and the gas collection apparatus. Volatiles that condense were collected in the receiver and analyzed separately. In all other respects the same procedure was followed as for the experiments done under reflux conditions.

Experiments involving two phases were done by the same procedures described above for single phase samples except that equal volumes $(2.0 \mathrm{~mL})$ of both TPB and aqueous acid phases were combined (except as noted in Appendix A). This size sample was chosen because it produced a volume of gas compatible with the apparatus. 


\section{II.3. Procedure for Constant Volume Decompositions}

The apparatus used for constant volume decomposition experiments is shown schematically in Figure 2. The apparatus consists of a $30 \mathrm{~mL}, 316$ stainless steel reaction vessel connected by 0.25 " stainless tubing to a pressure sensing devi:e (MKS Baratron, Model 122A), rupture disk assembly, and separate outlets for connecdive! of a vacuun pump, gas-inlet system and for gas sampling. After loading the cylinder with a sampie of known mass and volume ( $4.0 \mathrm{~mL}$ for single phase experiments, $2.0 \mathrm{~mL}$ of each phase for two phase experiments unless otherwise specified), the cylinder was attached to the system with a Swagelok fitting and the entire apparatus was pressurized with two atmospheres of helium and allowed to stand for $20 \mathrm{~min}$ to test for leaks. After the leak test the system was evacuated and backfilled with helium ten times to eliminate air from the system. After the last filling with helium the pressure was established at one atmosphere and the system sealed by closing the valve to the vacuum outlet. The cylinder was immersed into an oil bath that was preheated to the desired reaction temperature and pressure readings were taken as a function of time until no further pressure increase was observed. At this point the cylinder was removed from the bath and allowed to cool to room temperature where the pressure was again recorded.

Experiments involving two phases were done by the same procedures described above for single phase samples except that $2.0 \mathrm{~mL}$ of both TPB and aqueous acid phases were combined. The sample size was chosen to be the same as those for the constant pressure experiments.

Gauge pressures are reported for the constant volume experiments. Measured values were adjusted by substracting $1 \mathrm{~atm}$ pressure and correcting actual gas temperatures to $25^{\circ} \mathrm{C}$.

\section{II.4. Reactant and Product Analyses}

After reaction was complete as determined by stabilization of the system volume (constant pressure experiments) or system pressure (constant volume experiments), a sample of the gas phase was taken for infrared, mass spectral, or gas chromatographic analysis. The former two methods were used initially for identification of the gaseous constituents. Estimation of the gas phase composition was done by gas chromatography. Gas chromatographic analyses were performed using two Hewlett-Packard Model 5890 chromatographs, each equipped with thermal conductivity detectors, and each connected to a Hewlett-Packard workstation. Samples were taken with a gas-tight syringe and were injected with the same syringe into the chromatograph. One of the chromatographs was equipped with a $1 / 8^{\prime \prime} \times 12 ' 5 \AA$ molecular sieves column and was used for determination of $\mathrm{N}_{2}, \mathrm{O}_{2}, \mathrm{NO}$, and $\mathrm{He}$; argon was used as the carrier gas. The following instrument parameters were used: inlet temperature, $120^{\circ} \mathrm{C}$; oven temperature, $32{ }^{\circ} \mathrm{C}$; detector temperature, $120^{\circ} \mathrm{C}$; and carrier gas flow rate, $60 \mathrm{~mL}$ min. The second chromatograph was equipped with a Porapak Q column and was used for determination of $\mathrm{CO}, \mathrm{CO}_{2}$, and $\mathrm{N}_{2} \mathrm{O}$. Instrument parameters for this chromatograph were: inlet temperature, $60^{\circ} \mathrm{C}$; oven temperature, $32^{\circ} \mathrm{C}$; and detector temperature, $60^{\circ} \mathrm{C}$. Helium was used as the carrier gas in this instrument with a flow rate of $25 \mathrm{~mL} / \mathrm{min}$. 
After completion of all gas sampling, the reaction vessel was removed from the apparatus and the mass of the residue(s) was determined. The composition of liquid phases were determined by a combination of titrametric and NMR spectroscopic methods. Water and acid content were determined in the same fashion as for the equilibrated starting ma erials. Butyl nitrate and butyl phosphates were determined by ${ }^{1} \mathrm{H}$ NMR spectroscopy (versus a $\mathrm{CH}_{2} \mathrm{Cl}_{2}$ internal standard). Residual nitric acid was determined in some instances by ${ }^{14} \mathrm{~N}$ NMR spectroscopy and in other cases by difference based upon the amount of DBP and MBP determined by ${ }^{31} \mathrm{P}$ NMR spectroscopy.

\section{RESULTS}

Table 1 gives a summary of the experimental conditions for thitty three experiments that will be discussed in this report. These experiments were designed to explore the effects of temperature, nitric acid concentration, and of $\mathrm{Zr}(\mathrm{IV})$ as a catalyst on the rate and extent of decomposition of TBP/nitric acid mixtures. Additional parameters that were also considered were single phase versus two phase $\mathrm{TBP} / \mathrm{HNO}_{3}$ mixtures, constant pressure versus constant volume conditions, and for constant pressure experiments, reflux versus distillation. Two single phase experiments were also done to examine the effect of reduced water content, which was achieved by purging the samples with an inert gas prior to thermolysis, on the rate and extent of decomposition. A complete set of analytical data for each sample prior to, and after, reaction is given in Appendix A. It should be noted that the carbon and nitrogen mass balances for these experiments are poor in some cases. We believe that this is due primarily to the small sample sizes and the resulting losses associated with sample handling, especially when separation of phases was required. Appendices B-O provide graphical comparisons of product distributions for related experiments. The next four sections will discuss the effects of temperature, acid concentration, $\mathrm{Zr}(\mathrm{IV})$ catalyst, and prereaction purge with an inert gas to reduce the water content on the rate and extent of decomposition. The fifth section attempts to account for the various products in terms of possible reactions.

Experimental reproducibility was studied by repeating five experiments (see Table 1). Some of these results are summarized graphically (see Figs. G-1 through G-4, J-1 through $\mathrm{J}-4$, and $\mathrm{N}-1$ through $\mathrm{N}-3$ ). Generally, final gas volumes, pressure, and composition were reproducible within $\pm 5 \%$. Residual liquids and solids and, therefore, overall material balance estimate were less accurate because of the small quantities involved.

\section{III.1. Effect of temperature}

Appendices G-L give graphical summaries of the effects of temperature for reactions of TBP that is $6 \mathrm{M}$ in nitric acid under the following conditions: single phase, constant pressure, distillation $(\mathrm{G})$; single phase, constant pressure, reflux $(\mathrm{H})$; single phase, constant volume (I); two phase, constant pressure, distillation $(J)$; two phase, constant pressure, reflux (K); and two phase, constant volume (L). Reactions were conducted at bath temperatures of $130^{\circ} \mathrm{C}, 150^{\circ} \mathrm{C}$, and $180^{\circ} \mathrm{C}$ in all cases. The sample temperature was monitored for constant pressure samples. Under reflux conditions the sample temperature rose rapidly to an equilibrium value, which was always somewhat less than the bath 
WSRC-RP-95-259

temperature. This is attributed to the substantial loss of heat to the condenser. Under distillation conditions the sample temperature quickly rose to approximately the bath temperature. The actual sample temperature was not measured for constant volume experiments but it is expected to be nearly equal to the bath temperature. The extent of reaction, as measured by the amount of gas evolved per unit quantity of TBP, also increased with temperature for all but the distillation condition. Under distillation conditions the total amount of gas produced was greater at $130{ }^{\circ} \mathrm{C}$ with the amount produced at $150^{\circ} \mathrm{C}$ and $180^{\circ} \mathrm{C}$ being nearly equal and somewhat less than that at the lowest temperature. This effect most likely results from the rapid distillation of nitric acid from the mixture at $150^{\circ} \mathrm{C}$ and at $180^{\circ} \mathrm{C}$ because of rapid heat transfer from the bath to the sample. If nitric acid is driven from the reaction vessel faster than it can react with TBP and its hydrolysis products then the extent of reaction will be reagent limited.

\section{III.2 Effect of nitric acid concentration}

Appendices B-E give graphical summaries of the effects of nitric acid concentration on TBP reactions at $150^{\circ} \mathrm{C}$ under the following conditions: single phase, constant pressure, distillation (B); single phase constant volume (C); two phase, constant pressure, distillation (D); and two phase, constant volume (E). Reactions were conducted using TBP phases equilibrated with either $3 \mathrm{M}$ or $6 \mathrm{M}$ nitric acid in all cases.

More gaseous moles are generated per mole of TBP charged for $6 \mathrm{M} \mathrm{HNO}_{3}$ in all cases except Appendix D (two-phase, constant pressure, distillation) where a slightly higher amount of gas was generated using $3 \mathrm{M} \mathrm{HNO}_{3}$, apparently from higher $\mathrm{NO}$ production in this case. By far, however, the greatest gas generation occurred for the cases in Appendix $\mathrm{E}$ (two-phases, constant volume) where $6 \mathrm{M} \mathrm{HNO}_{3}$ generates over twice as much gas per mole of TBP charged as does the $3 \mathrm{M}$ case. This effect is probably due to the fact that the extent of reaction is not $\mathrm{HNO}_{3}$ limited in this case. About twice as much $\mathrm{HNO}_{3}$ is consumed compared to $3 \mathrm{M} \mathrm{HNO}_{3}$. Also, twice as much $\mathrm{CO}_{2}$ is produced and the $\mathrm{CO}$, $\mathrm{NO}$ and $\mathrm{N}_{2} \mathrm{O}$ concentrations are significantly higher. Both the increasing pressure, by reducing the rate of $\mathrm{HNO}_{3}$ evaporation, and the excess acid in the second phase can contribute to this effect.

Comparing the residual liquid species (Fig C.4 and E.4), we see that the relative effect of acid is about the same for the single phase cases, for the constant pressure, distillation cases versus the constant volume cases. However, the overall extent of reaction is greater in the constant volume cases, especially in the $6 \mathrm{M}$ cases, where about twice as much gas is produced at constant volume.

\section{III.3. Effect of $\mathrm{Zr}(\mathrm{IV})$}

Appendices $\mathrm{M}$ and $\mathrm{N}$ provide graphical summaries of the effects of presence of $\mathrm{Zr}^{4+}$ on single phase reactions of TBP in $6 \mathrm{M}$ nitric acid at $150^{\circ} \mathrm{C}$ with either constant pressure or constant volume conditions. For the constant pressure experiments (Appendix $M$ ), the experiments were conducted in the distillation mode (see Section II). In the experiments 
with $\mathrm{Zr}^{4+}$, water and nitric acid saturated TBP was equilibrated with zirconyl nitrate hydrate solid. The resulting TBP extract was then reacted at either constant pressure, or constant volume conditions. For the constant pressure distillation experiments (Appendix $\mathrm{M})$, the presence of $\mathrm{Zr}^{4+}$ slightly increases the extent of reaction, i.e. the total amount of gas produced, without significant changes in the gas composition (Figs. M-1 and M-2). The composition of the liquid species, however, is significantly affected as can be seen from Fig. M-3. For the constant volume experiments, however, the presence of $\mathrm{Zr}^{4+}$ produces an overshoot in the pressure transient (i.e. gas production rate), as can be seen in Figure N-3. This phenomenon was observed only for constant volume experiments in the presence of $\mathrm{Zr}^{4+}$, and was clearly repeatable, as evidenced by behavior shown for Experiments \#163 and 166 (Fig. $\mathrm{N}-3$ ). While the final amount of gas produced is essentially unaffected by the presence of $\mathrm{Zr}^{4+}$, the gas composition appears to be significantly affected with a relatively large increase in the amount of nitrogen produced and a total absence of nitric oxide (Fig. N-2).

\section{III.4. Effect of Reduced Water Content}

Appendix F provides graphical representation of the effects of reduced water content on single phase reactions of TBP in $3 \mathrm{M}$ nitric acid at $150^{\circ} \mathrm{C}$ with constant pressure, distillation conditions. The water in the initial reactants was removed by purging the initial water and nitric acid saturated TBP with helium for an extended period. The $3 \mathrm{M}$ value represents the final nitric acid concentration in the reactants for the experiments with (\#136) and without (\#147A) water. The experiments were conducted to examine the effect of routine purging operations on aged reactants which may reside in the process vessels for extended periods. As can be seen from Fig. F-1 removal of water from the reactants will significantly increase the extent of the reaction, i.e. the amount of gas produced per mole TBP charged; the normalized gas production (mole/mole TBP) nearly doubles. The normalized gas composition is also affected (Fig. F-2) with significant increases in the fractions of nitrogen and nitric oxide and corresponding reductions in the fraction of $\mathrm{CO}$ and $\mathrm{CO}_{2}$. The $\mathrm{N}_{2} \mathrm{O}$ fraction is slightly reduced. The effect of purging on the liquid product species is shown in Figure F-4; the resulting amounts of water, DBP, and $\mathrm{BuONO}_{2}$ per mole TBP charged are all reduced.

\section{III.5. Possible Origins of Products}

It is impossible to suggest precise mechanisms of formation for all of the products, gaseous and condensed phase, that have been observed as products of the decomposition of TPB. It would be even more impossible to account for the apparent stoichiometries that are observed. It is possible, however, to suggest how some of the observed products may arise based upon literature precedents.

Formation of DBP and MBP may each arise by two pathways, either acid catalyzed hydrolysis of the P-O bond to give 1-butanol (reaction 1) or transesterification with nitric acid to give butyl nitrite (reaction 2). Butyl nitrite is observed as a product, whereas 1butanol is not in most cases. These compounds are related by the equilibrium shown in reaction 3. 


$$
\begin{aligned}
& \mathrm{Bu}_{3-x}(\mathrm{HO})_{x} \mathrm{PO}+\mathrm{H}_{2} \mathrm{O} \rightarrow \mathrm{Bu}_{3-(x+1)}(\mathrm{HO})_{x+1} \mathrm{PO}+\mathrm{BuOH} \\
& \mathrm{Bu}_{3-x}(\mathrm{HO})_{x} \mathrm{PO}+\mathrm{HNO}_{3} \rightarrow \mathrm{Bu}_{3(x+1)}(\mathrm{HO})_{x+1} \mathrm{PO}+\mathrm{BuONO}_{2} \\
& \mathrm{BuOH}+\mathrm{HONO}_{3} \rightarrow \mathrm{BuONO}_{2}+\mathrm{H}_{2} \mathrm{O}
\end{aligned}
$$

Studies by Moffat and Thompson ${ }^{7}$ suggest that (2) is more important than (1) and that the presence of $\mathrm{Zr}(\mathrm{IV})$ in the TBP phase can accelerate the decomposition of TBP. The literature states that hydrolysis of nitrate esters is slow, ${ }_{1}^{8}$ even at elevated temperatures, so that establishment of equilibrium in (3) may not occur prior to decomposition of the nitrate ester or reaction of 1-butanol, if indeed it is ever present. In any case it is the oxidation of these two species that is responsible for the production of gases.

The complexity of nitrogen chemistry is such that it is impossible to predict stoichiometric reactions for formation of the gaseous products. Nitric acid oxidations are generally subject to autocatalysis so there is usually an induction period unless nitrite or nitrous acid has been added initially." Several species are believed to be involved in "nitric acid" oxidations, including $\mathrm{NO}, \mathrm{NO}_{2}, \mathrm{~N}_{2} \mathrm{O}_{3}$ and $\mathrm{HONO}$. The following reactions account for the autocatalysis and for the production of the actual oxidants.

$$
\begin{aligned}
& 3 \mathrm{HONO} \rightarrow \mathrm{HONO}_{2}+2 \mathrm{NO}+\mathrm{H}_{2} \mathrm{O} \\
& \mathrm{HONO}_{2}+\mathrm{HONO}=\mathrm{N}_{2} \mathrm{O}_{4}+\mathrm{H}_{2} \mathrm{O} \\
& \mathrm{N}_{2} \mathrm{O}_{4}=2 \mathrm{NO}_{2} \\
& \mathrm{~N}_{2} \mathrm{O}_{4}+2 \mathrm{NO}+2 \mathrm{H}_{2} \mathrm{O}=4 \text { HONO} \\
& \mathrm{NO}+\mathrm{NO}_{2}=\mathrm{N}_{2} \mathrm{O}_{3}=\mathrm{NO}^{+}+\mathrm{NO}_{2}^{-}
\end{aligned}
$$

Nitric oxide, nitrous oxide and nitrogen are typical reduction products from nitric acid oxidations; however, it is well established that $\mathrm{N}_{2}$ can only arise from nitric acid provided negative oxidation states are first formed so that they may be oxidized by nitric acid. Nitrous oxide probably arises from a related pathway. The nitrogen species with a negative oxidation state that is most likely to be formed in TBP decomposition reactions is hydroxyl amine $\left(\mathrm{H}_{2} \mathrm{NOH}\right)$, which can be formed by hydrolysis of oximes. Oximes can form by tautomerization of nitroso alkanes $\left(\mathrm{RCH}_{2} \mathrm{NO}\right)$, which are known products of both $\mathrm{NO}$ and $\mathrm{NO}^{+}$with several types of organic molecules, including alcohols and carbonyl compounds. ${ }^{10}$ This reaction sequence is shown is shown in (9)-(10).

$\mathrm{RCH}_{2} \mathrm{NO} \rightarrow \mathrm{RCH}=\mathrm{NOH}$

$\mathrm{RCH}=\mathrm{NOH}+\mathrm{H}_{2} \mathrm{O} \rightarrow \mathrm{RCHO}+\mathrm{H}_{2} \mathrm{NOH}$ 
The oxidation of hydroxyl amine has been investigated under acidic conditions. ${ }^{11,12}$ Reaction is only observed at sufficiently high nitric acid concentrations and autocatalysis by nitrous acid was observed. The distribution of products changes markedly with hydroxylamine concentration with nitrogen being observed only at low hydroxylamine concentrations and the yield of nitrous oxide increasing at higher hydroxylamine concentrations. Hydroxyl amine has not been detected as a product in our study but this is not surprising given its high reactivity and probable low concentration.

There are several possible routes to oxidation products of 1-butanol. Elimination of HONO from the nitrite ester can lead to aldehyde (Â-elimination, reaction 11 ) or olefin ( $\beta$ elimination, reaction 12 ). Homolytic cleavage of the $\mathrm{N}-\mathrm{OC}$ bond is also a possibility (reaction 13)

$\mathrm{PrCH}_{2} \mathrm{ONO}_{2} \rightarrow \mathrm{PrCHO}+\mathrm{HONO}$

$\mathrm{EtCH}_{2} \mathrm{CH}_{2} \mathrm{ONO} \rightarrow \mathrm{EtCH}=\mathrm{CH}_{2}+\mathrm{HONO}$

$\mathrm{PrCH}_{2} \mathrm{ONO} \rightarrow \mathrm{PrCH}_{2} \mathrm{O}+\mathrm{NO}_{2}$

Reaction (12) must have a higher activation energy than the other two, or any other reactions that may be involved, since olefin was not observed as a product in this study.

Oxidation of aldehydes and alcohols can occur by several pathways. ${ }^{10}$ Degradation of carboxylic acids by nitric acid to give smaller carboxylic acids, i.e., butanoic to propanoic, and carbon monoxide and carbon dioxide is well known although the precise mechanisms are apparently not so well established.

\section{CONCLUSIONS AND RECOMMENDATIONS}

A total of thirty three experiments have been conducted at various temperatures, and acid concentrations for both single (organic) phase and two-phase systems in the presence and absence of $\mathrm{Zr}^{4+}$ (saturated). Experiments were conducted under both constant pressure (ambient) and constant volume conditions.

The obvious conclusions are that an increase in temperature or acid concentration results in an increased rate and quantity of gas produced. The addition of $\mathrm{Zr}^{4+}$ had a slight effect on the amount of gas produced under constant pressure conditions, but had essentially no effect on total gas production under constant volume conditions. The latter experiments did show unusual overshoots in pressure that suggest the initial formation of gaseous products that were subsequently consumed. The origin of this phenomenon is not known although reaction (7) would produce this result. The effect of $\mathrm{Zr}^{4+}$ observed here is not inconsistent with the constant pressure experiments. This is true since the greater the gas pressure, the rate of both gas phase and solution phase reactions will increase because of the higher concentrations. The effect of such pressure overshoots in a sealed, or poorly ventilated, storage tank cannot be estimated on the basis of these data. 
A greater nitric acid/water ratio in the TBP, which was achieved in our experiments by inert gas purging, also increases the amount of gas produced.

Although some differences in gaseous product ratios have been observed, we are unable to provide an explanation for these differences at the present time. It might be possible to proviais an interpretation of these differences if more concrete mechanistic information were available.

It is noteworthy that no "run-away" reactions were observed in these studies and as such they may not be considered as particularly good models for the reactions of Red Oil. There are several possible reasons why they do not show the same behavior as Red Oil. First, the small size of the sample and the isothermal conditions used in these studies may have prevented self-heating. Second, the absence of nitrite, which would be present in Red Oil due to radiolytic decomposition of nitrate ${ }^{13-16}$, which would reduce the rate of the initial reaction may be significant. Finally, the presence of actinide ions may also be important in determining the reactivity of Red Oil. The effect of added nitrite and the use of nonisothermal conditions can be readily tested experimentally.

Although oxidations by nitric acid are very complicated mechanistically, the complexity of the TBP system studied here makes any definitive statement of reaction pathways impossible. If, however, it is concluded that a more detailed mechanistic approach to the origin of products is desired, then the reactions of several known, or suspected, intermediates such as butyl nitrate, butanol, butyraldehyde, and butyric acid with nitric acid could be undertaken.

Only a small fraction of factors affecting gas production and, potentially, safety issues have been studied. Sample aging and gamma irradiation effects, for example, should be examined. The sample sizes were small in our study due to safety considerations, and reacting samples were alway well mixed. Larger unmixed samples may undergo localized heating that could cause runaway reactions; these factors should also be studied experimentally under comparable conditions.

Zirconium (IV) was shown to affect the reaction path. The effect of uranyl nitrate should also be evaluated.

Higher reactivities resulted from He purging to remove water prior to decomposition, but no experiments were conducted to assess the effects of oxygen and ozone. So the results presented here may underestimate the hazards associated with the use of air-lift pumps since oxygen partial pressures over TBP solutions are higher under plant conditions, and since oxygen will almost certainly affect the reaction path significantly. Ozone, of course, is formed whenever dissolved oxygen is irradiated; this factor should also be examined experimentally.

In a few experiments (see Fig. N-4), we observed higher intermediate pressures than existed at the end of the experiment. This behavior was replicated in two independent tests, and it may have important implications for safety, if substantially higher initial 
WSRC-RP-95-259

overpressure can occur as indicated. Also, the effects of sample size, mixing, and the other variables discussed above should be carefully assessed in regard to this behavior.

\section{REFERENCES}

1. Iv. L. Hyder, "Safe Handling of TBP and Nitrates in the Nuclear Process Industry," Westinghouse Savannah River Company, WSRC-TR-94-0372 (July 1994).

2. D. O. Campbell and J. C. Mailen, "The Red Oil Problem and Its Impact on Purex Safety," Oak Ridge National Laboratory, ORNL/TM-10798 (June 1988).

3. Fauske and Associates, "Tributyl Phosphate-Nitric Acid Reaction and Vent Requirement," FAI/94-68, July, 1994.

4. G. S. Barney and T. D. Cooper, "The Chemistry of Tributyl Phosphate at Elevated Temperatures in the Plutonium Finishing Plant Process Vessels," WHC-EP-0737, March, 1994.

5. P. L. Gordon, C. O'Dell, and J. G. Watkin, "Synthesis and Energetic Content of Red Oil," Joumal of Hazardous Materials (to appear); private communication with D. W. Tedder (August 31, 1994).

6. T. G. Howard, "Consequences of a Tomsk-Type Incident in F-Canyon," SRT-PST940017, January 25, 1994.

7. A. J. Moffat and R. D. Thompson, "The Chemical Stability of Tributyl Phosphate in Some Nitrate and Chloride Systems," J. Inorg. Nucl. Chem., 16, 465 (1961).

8. C. R. Noller, "Chemistry of Organic Compounds," Second Edition, W. B. Saunders Co., Philadelphia, 1957, p. 109.

9. G. Bazsa and I. R. Epstein, "Kinetics and Mechanism of Autocatalytic Nitric Acid Oxidations," Comments Inorg. Chem., 5, 57 (1986).

10. Y. Ogata, in "Oxidations in Organic Chemistry," Part C, ed. W. S. Trahanowsky, Academic Press, 1978, p. 296.

11. D. C. Moews and L. F. Audrieth, "Autooxidation of Hydroxylamine," J. Inorg. Nucl. Chem., 21, 242 (1959).

12. J. R. Pembridge and G. Stedman, "Kinetics, Mechanism, and Stoichiometry of the Oxidation of Hydroxylamine by Nitric Acid," J. Chem. Soc., Dalton Trans. 1658 (1979).

13. C. D. Kalkar and V. M. Raut, "Efficiency of transfer of stored energy from gammairradiated $\mathrm{NaCl}$ in aqueous ammonium nitrate solutions," Appl. Radiat. Isot, 43(10) (Oct 1992) p 1207-1210. 
14. T. Soga and K. Furkawa, "Molecular beam effects in energetic particle induced decomposition of potassium nitrate," J. Nucl. Sci. Technol., 26(6) (June 1989) p 639641.

15. S.F. Patil and $\therefore$ G. Bedekar, "Effect of particle size of nitrate and oxides as heterphase impurity on tì iadiation decomposition of pure and barium oxide doped potassium nitrate crystals," Radiat. Phys. Chem., 29(2) (1987) p. 121-125.

16. R. Nieder and W. Straeter, "Radiolysis processes in the water-steam circuit of the experimental nuclear reactor (AVR) at Juelich," VGB Kraftwerkstech 66(12) (Dec 1986), p. 1167-1171. 
TABLE 1

WSRC-RP-95-259

\section{LIST OF COMPLETED EXPERIMENTS}

\section{Single Phase}

1. Constant Pressure

Exp. No Bath Temp Acid in TBP Refluxing/Distillation $\underline{\mathrm{Z}}^{4+}$

$\begin{array}{lllll}136 & 150^{\circ} \mathrm{C} & 3 \mathrm{M} & \mathrm{D} & - \\ 137 & 150^{\circ} \mathrm{C} & 6 \mathrm{M} & \mathrm{D} & - \\ 139 & 130^{\circ} \mathrm{C} & 6 \mathrm{M} & \mathrm{D} & - \\ 140 & 150^{\circ} \mathrm{C} & 6 \mathrm{M} & \mathrm{D} & \mathrm{Zr}^{4+} \\ 141 & 180^{\circ} \mathrm{C} & 6 \mathrm{M} & \mathrm{D} & - \\ 144 & 150^{\circ} \mathrm{C} & 6 \mathrm{M} & \mathrm{R} & - \\ 145 & 130^{\circ} \mathrm{C} & 6 \mathrm{M} & \mathrm{R} & - \\ 146 & 180^{\circ} \mathrm{C} & 6 \mathrm{M} & \mathrm{R} & -\end{array}$

2. Constant Volume

Exp. No Bath Temp $\quad \underline{\text { Acid in TBP }} \quad \underline{\mathrm{Zr}}^{4+}$

$\begin{array}{llll}134 & 150^{\circ} \mathrm{C} & 3 \mathrm{M} & - \\ 135 & 150^{\circ} \mathrm{C} & 6 \mathrm{M} & - \\ 142 & 130^{\circ} \mathrm{C} & 6 \mathrm{M} & - \\ 143 & 180^{\circ} \mathrm{C} & 6 \mathrm{M} & - \\ 163 & 150^{\circ} \mathrm{C} & 6 \mathrm{M} & \mathrm{Zr}^{4+}\end{array}$

\section{Two Phase}

1. Constant Pressure

Exp. No Bath Temp $\underline{\text { Acid in TBP }}$ Refluxing/Distillation $\underline{\mathrm{Z}}^{4+}$

$\begin{array}{lll}150 & 150^{\circ} \mathrm{C} & 6 \mathrm{M} \\ 151 & 150^{\circ} \mathrm{C} & 3 \mathrm{M} \\ 152 & 150^{\circ} \mathrm{C} & 6 \mathrm{M} \\ 153 & 180^{\circ} \mathrm{C} & 6 \mathrm{M} \\ 154 & 130^{\circ} \mathrm{C} & 6 \mathrm{M} \\ 159 & 180^{\circ} \mathrm{C} & 6 \mathrm{M} \\ 160 & 130^{\circ} \mathrm{C} & 6 \mathrm{M} \\ 161 & 150^{\circ} \mathrm{C} & 6 \mathrm{M}\end{array}$

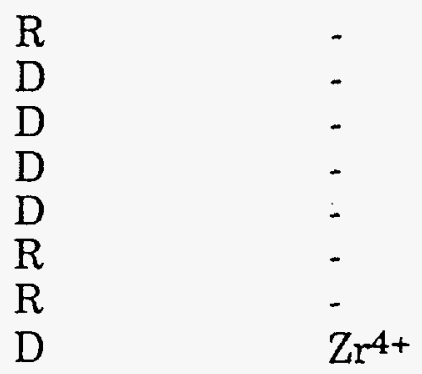




\section{Constant Volume}

$\begin{array}{cccc}\text { Exp. No } & \text { Bath Temp } & \text { Acid in TBP } & \text {. } \\ & 150^{\circ}{ }^{\circ+} & 6 \mathrm{M} & - \\ 155 & 150^{\circ} \mathrm{C} & 3 \mathrm{M} & - \\ 156 & 130^{\circ} \mathrm{C} & 6 \mathrm{M} & - \\ 157 & 180^{\circ} \mathrm{C} & 6 \mathrm{M} & - \\ 158 & 150^{\circ} \mathrm{C} & 6 \mathrm{M} & \mathrm{Zr}^{4+} \\ 162 & & & \end{array}$

\section{Additional Experiments}

1. Single Phase with No Water TBP (Purged Samples)

Exp. No Bath Temp Acid in TBP Const. Pressure/Nolume $\underline{\mathrm{Z}}^{4+}$

\begin{tabular}{lrr}
$147 \mathrm{~A}$ & $150^{\circ} \mathrm{C}$ & $\sim 3 \mathrm{M}$ \\
$147 \mathrm{~B}$ & $150^{\circ} \mathrm{C}$ & $\sim 3 \mathrm{M}$ \\
& & \\
\multicolumn{2}{r}{ Reproducibility } & Experiments
\end{tabular}

Exp. No Checking Exp. No

$\begin{array}{ll}148 \mathrm{~A} & 137 \\ 148 \mathrm{~B} & 136 \\ 148 \mathrm{C} & 140 \\ 165 & 154 \\ 166 & 163\end{array}$




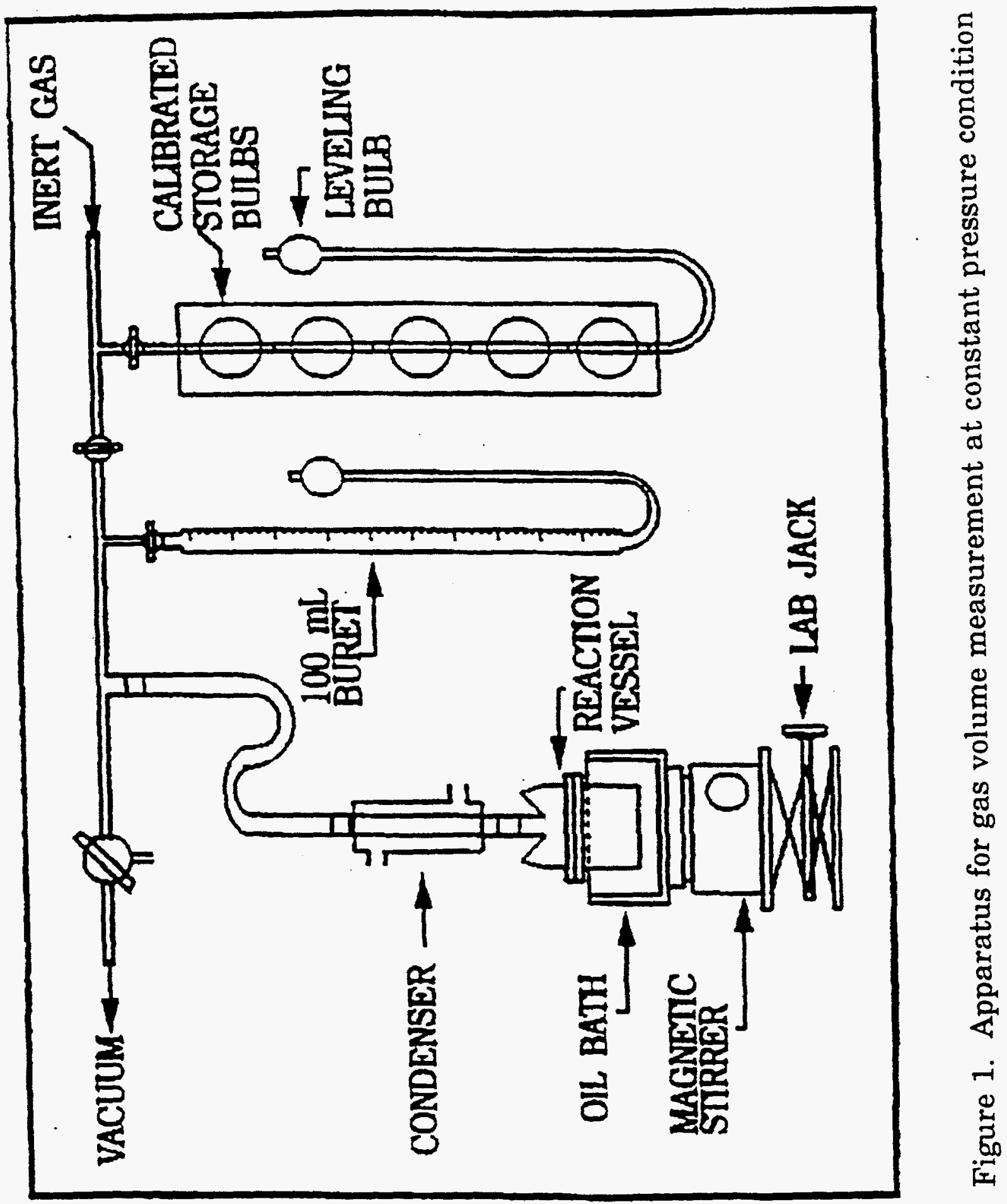


WSRC-RP-95-259

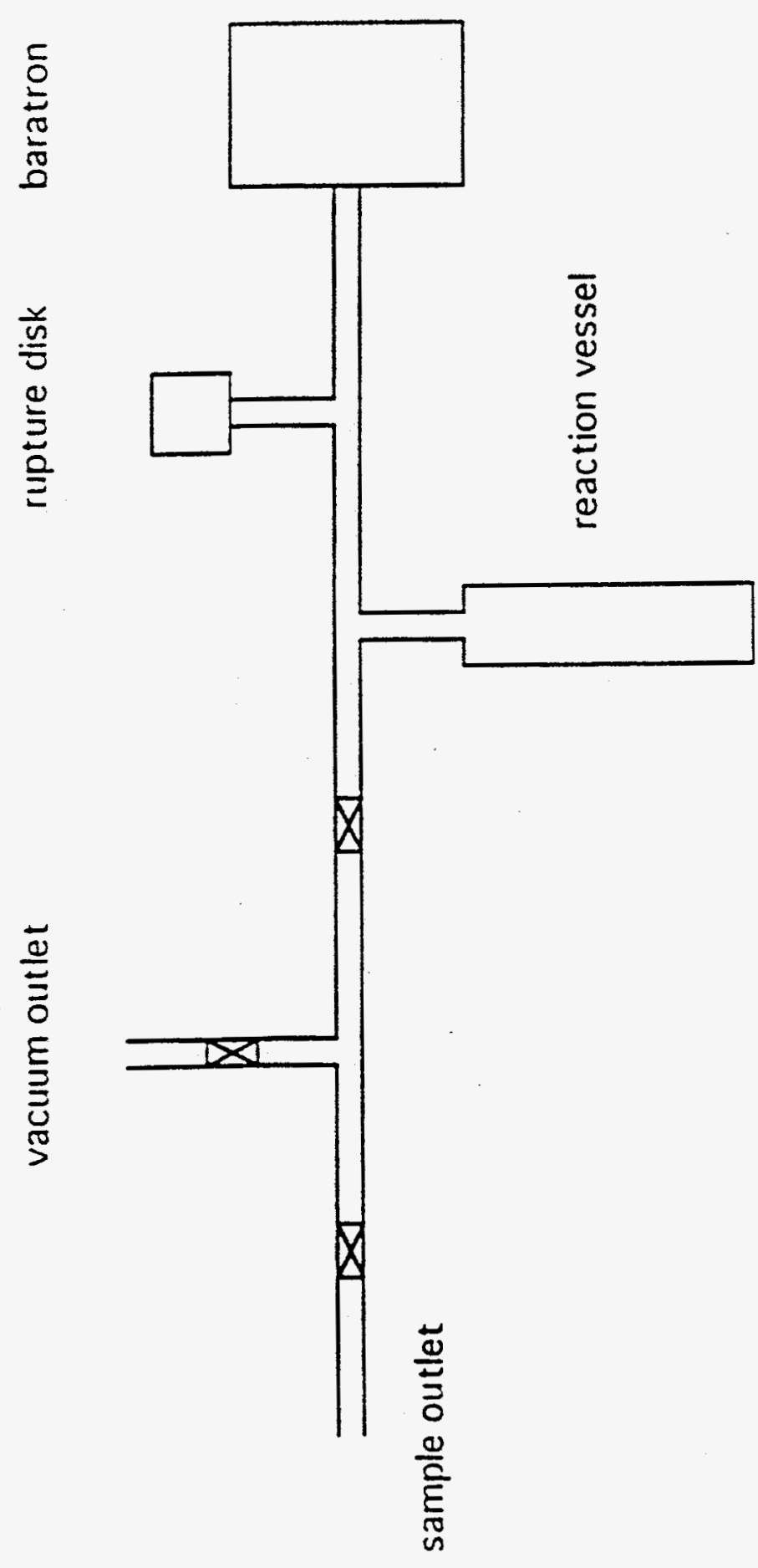

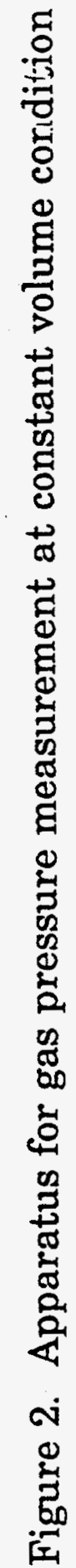


WSRC-RP-95-259

\section{APPENDIX A}

\section{ANALYTICAL DATA}

This appendix provides a listing of analytical data for each of the thirty three experiments conducted in this investigation prior to, and after, reaction. A summary of experimental conditions for all experiments is given in Table 1 . 
WSRC-RP-95-259

\section{Results of Experiment No. 134 (Const. Volume, Single Phase)}

Reaction Conditions:

Bath Temperature: $\quad 150^{\circ} \mathrm{C}$

Sample Volume: $\quad 5 \mathrm{~mL}$

Acid Concentration: $\quad 3 \mathrm{M}$

Before Reaction:

Sample Weight: $5.10 \mathrm{~g}$

[H+]:

$15.0 \mathrm{mmol}(3.00 \mathrm{M})$

$\left[\mathrm{H}_{2} \mathrm{O}\right]$ :

$7.42 \mathrm{mmol}(1.48 \mathrm{M})$

[TBP]:

$14.20 \mathrm{mmol}(2.84 \mathrm{M})$

Helium

\section{After Reaction:}

Total Gas Pressure: $\quad 4.5$ atms $\left(25^{\circ} \mathrm{C}, 58 \mathrm{~mL}\right.$ volume $)$

$\begin{array}{lllllllll}\text { Gas Composition: } & \mathrm{N}_{2} & \mathrm{O}_{2} & \mathrm{CO} & \mathrm{CO}_{2} & \mathrm{NO} & \mathrm{N}_{2} \mathrm{O} & \mathrm{He}\end{array}$

$\begin{array}{llllllll}\text { total mol \% } & 23.7 & 0 & 17.7 & 28.5 & 5.6 & 10.5 & 16.1\end{array}$

normalized mol \% $\quad 27.6 \quad 0 \quad 20.6 \quad 33.1 \quad 6.5 \quad 12.2$

Residue Weight: $4.65 \mathrm{~g}$ (weight loss $=0.45 \mathrm{~g}$ )

Residue Composition: (by acid-base titration, Karl-Fisher titration and ${ }^{1} \mathrm{H}$ NMR)

$\left[\mathrm{H}^{+}\right]$: $\quad 8.79 \mathrm{mmol}(1.89 \mathrm{M})$

$\left[\mathrm{H}_{2} \mathrm{O}\right]: \quad 12.00 \mathrm{mmol}(2.58 \mathrm{M})$

[TBP]: $\quad 9.94 \mathrm{mmol}(2.23 \mathrm{M})$

[DBP]: $\quad 4.26 \mathrm{mmol}(0.95 \mathrm{M})$

[BuONO 2 ]: $\quad 3.00 \mathrm{mmol}(0.65 \mathrm{M})$

Material Balance

C lost from residue: $\quad 5.74 \mathrm{mmol}$

C present in gas: $\quad 5.04 \mathrm{mmol}$

$\mathrm{N}$ lost from residue: $7.47 \mathrm{mmol}$

$\mathrm{N}$ present in gas: $\quad 9.20 \mathrm{mmol}$

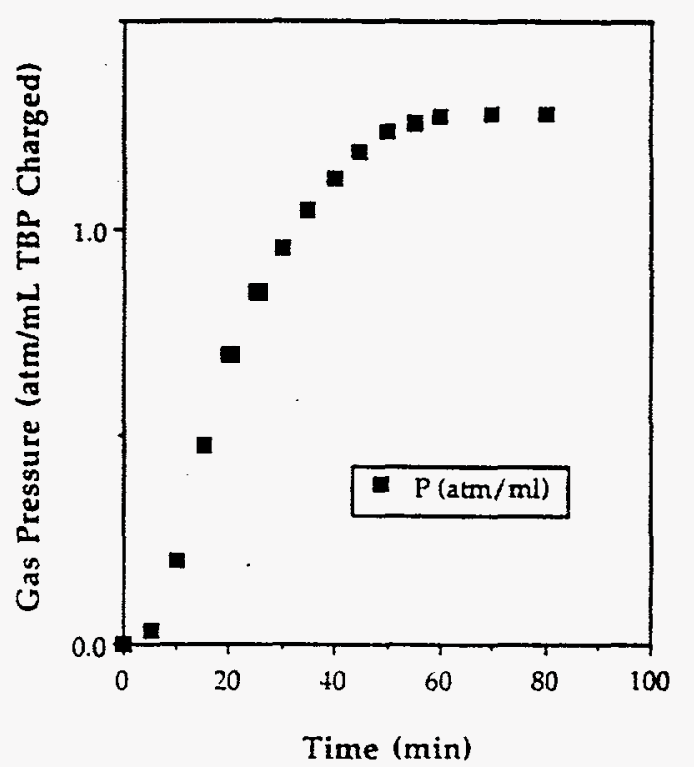




\section{Results of Experiment No. 135 (Const. Volume, Single Phase)}

Reaction Conditions:

Bath Temperature: $\quad 150^{\circ} \mathrm{C}$

Sample Volume:

Before Reaction:

Sample Weight: $5.10 \mathrm{~g}$

$\left[\mathrm{H}^{+}\right]$:

$29.25 \mathrm{mmol}(5.85 \mathrm{M})$

$\left[\mathrm{H}_{2} \mathrm{O}\right]$ :

$9.40 \mathrm{mmol}(1.88 \mathrm{M})$

[TBP]:
Acid concentration:

Atmosphere:
$6 \mathrm{M}$

Helium

After Reaction:

Total Gas Pressure:

10.3 atms $\left(25^{\circ} \mathrm{C}, 58 \mathrm{~mL}\right.$ volume $)$

Gas Composition:

$\begin{array}{llllllll}\mathrm{N}_{2} & \mathrm{O}_{2} & \mathrm{CO} & \mathrm{CO}_{2} & \mathrm{NO} & \mathrm{N}_{2} \mathrm{O} & \mathrm{He}\end{array}$

total mol \%

$7.7 \quad 0$

25.1

32.3

7.7

$10.4 \quad 9.1$

$\begin{array}{lllllll}\text { normalized mol \% } & 9.3 & 0 & 30.2 & 38.8 & 9.3 & 12.5\end{array}$

Residue Weight: $\quad 4.25 \mathrm{~g}$ (weight loss $=0.85 \mathrm{~g}$ )

Residue Composition (from acid-base titration, Karl-Fisher titration and ${ }^{1} \mathrm{H}$ NMR)

$\left[\mathrm{H}^{+}\right]$:

$12.79 \mathrm{mmol}(3.01 \mathrm{M})$

$\left[\mathrm{H}_{2} \mathrm{O}\right]:$

$16.83 \mathrm{mmol}(3.96 \mathrm{M})$

[TBP]:

$7.02 \mathrm{mmol}(1.56 \mathrm{M})$

[DBP]:

$6.21 \mathrm{mmol}(1.38 \mathrm{M})$

$\left[\mathrm{BuONO}_{2}\right]: \quad 3.51 \mathrm{mmol}(0.83 \mathrm{M})$

Material Balance

C lost from residue: $\quad 10.8 \mathrm{mmol}$

$\mathrm{C}$ present in gas: $\quad 16.9 \mathrm{mmol}$

$\mathrm{N}$ lost from residue: $19.2 \mathrm{mmol}$

$\mathrm{N}$ present in gas: $\quad 12.9 \mathrm{mmol}$

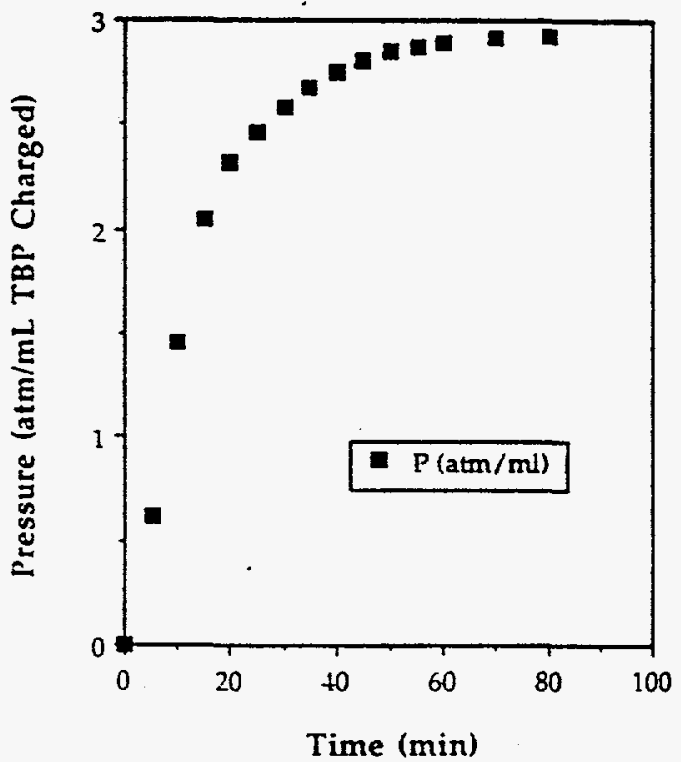




\section{Results of Experiment No. 136 (Const. Pressure, Single Phase)}

\section{Reaction Conditions:}

Bath Temperature: $\quad 150^{\circ} \mathrm{C}$

Acid concentration: $\quad 3 \mathrm{M}$

Sample Volume:

$5 \mathrm{~mL}$

Atmosphere:

Helium

Refluxing/Distillation D

\section{Before Reaction:}

Sample Weight: $5.13 \mathrm{~g}$

$\left[\mathrm{H}^{+}\right]$: $15.00 \mathrm{mmol}(3.00 \mathrm{M})$

$\left[\mathrm{H}_{2} \mathrm{O}\right]:$

$7.40 \mathrm{mmol}(1.48 \mathrm{M})$

[TBP]:

$14.20 \mathrm{mmol}(2.84 \mathrm{M})$

\section{After Reaction:}

Total Gas Volume: $\quad 165 \mathrm{~mL}\left(25^{\circ} \mathrm{C}, 1 \mathrm{~atm}\right)$

Gas Composition: $\quad \begin{array}{lllllll}\mathrm{N}_{2} & \mathrm{O}_{2} & \mathrm{CO} & \mathrm{CO}_{2} & \mathrm{NO} & \mathrm{N}_{2} \mathrm{O} & \mathrm{He}\end{array}$

$\begin{array}{llllllll}\text { total } \mathrm{mol} \mathrm{\%} & 3.5 & 0 & 18.0 & 29.8 & 31.4 & 8.4 & 3.5\end{array}$

$\begin{array}{lllllll}\text { normalized mol \% } & 3.8 & 0 & 19.8 & 32.7 & 34.5 & 9.2\end{array}$

Residue Weight: $4.10 \mathrm{~g}$ (weight loss $=1.03 \mathrm{~g}$ )

Residue Composition: (by acid-base titration, Karl-Fisher titration and ${ }^{1} \mathrm{H}$ NMR)

$\left[\mathrm{H}^{+}\right]$: $\quad 9.37 \mathrm{mmol}(2.29 \mathrm{M})$

$\left[\mathrm{H}_{2} \mathrm{O}\right]: \quad 3.15 \mathrm{mmol}(0.77 \mathrm{M})$

[TBP]: $\quad 9.42 \mathrm{mmol}(2.30 \mathrm{M})$

[DBP]: $\quad 4.78 \mathrm{mmol}(1.17 \mathrm{M})$

[BuONO 2$]: \quad 0.22 \mathrm{mmol}(0.05 \mathrm{M})$

[MeCOOH]: $0.22 \mathrm{mmol}(0.05 \mathrm{M})$

Condensate Compositions:

Aqueous Phase $(0.41 \mathrm{~g})$

$\left[\mathrm{H}^{+}\right]$:

$2.21 \mathrm{mmol}(5.52 \mathrm{M})$

Organic Phase $(0.30 \mathrm{~g})$

$\left[\mathrm{BuONO}_{2}\right]: 2.39 \mathrm{mmol}(8.20 \mathrm{M})$

\section{Material Balance}

$\mathrm{C}$ lost from residue: $8.24 \mathrm{mmol}$

C present in Gas: $\quad 3.55 \mathrm{mmol}$

$\mathrm{N}$ lost from residue: $5.81 \mathrm{mmol}$

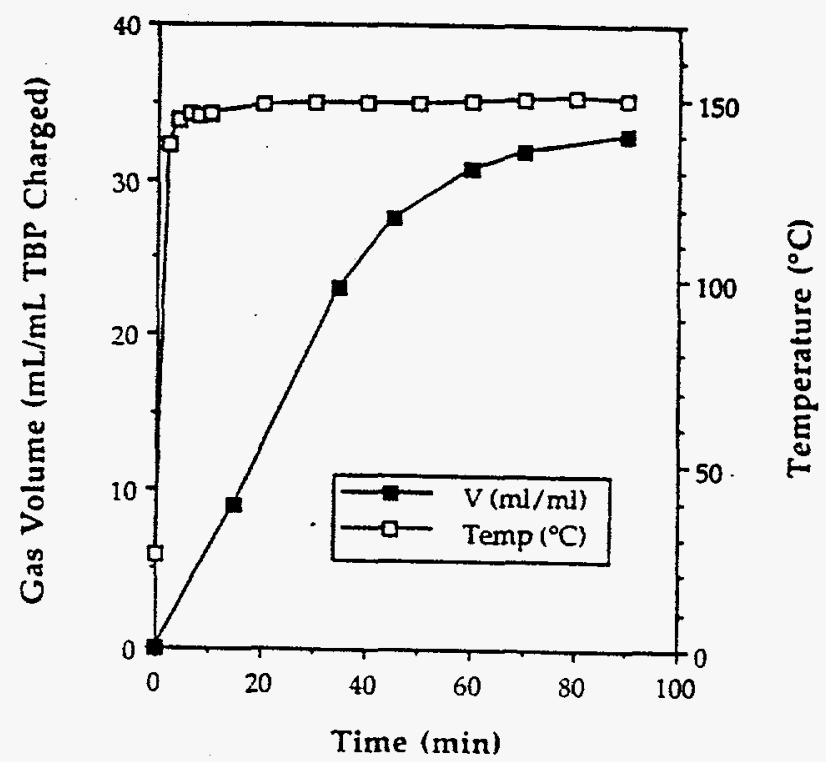

$\mathrm{N}$ present in Gas: $\quad 4.10 \mathrm{mmol}$ 


\section{Results of Experiment No. 137 (Const. Pressure, Single Phase)}

\section{Reaction Conditions:}

Bath Temperature: $\quad 150^{\circ} \mathrm{C}$

Acid concentration: $\quad 6 \mathrm{M}$

Sample Volume:

$5 \mathrm{~mL}$

Atmosphere:

Helium

Refluxing/Distillation D

\section{Before Reaction:}

Sample Weight: $5.43 \mathrm{~g}$

$\left[\mathrm{H}^{+}\right]$:

$28.75 \mathrm{mmol}(5.75 \mathrm{M})$

$\left[\mathrm{H}_{2} \mathrm{O}\right]$ :

$9.40 \mathrm{mmol}(1.88 \mathrm{M})$

[TBP]:

$13.25 \mathrm{mmol}(2.65 \mathrm{M})$

\section{After Reaction:}

Total Gas Volume: $\quad 235 \mathrm{~mL}\left(25^{\circ} \mathrm{C}, 1 \mathrm{~atm}\right)$

$\begin{array}{lllllllll}\text { Gas Composition: } & \mathrm{N}_{2} & \mathrm{O}_{2} & \mathrm{CO} & \mathrm{CO}_{2} & \mathrm{NO} & \mathrm{N}_{2} \mathrm{O} & \mathrm{He}\end{array}$

$\begin{array}{llllllll}\text { total mol \% } & 3.4 & 0 & 14.3 & 30.1 & 27.6 & 7.0 & 1.6\end{array}$

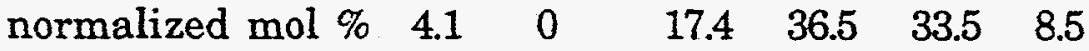

Residue Weight: $3.58 \mathrm{~g}$ (weight loss $=1.85 \mathrm{~g}$ )

Residue Composition: (by acid-base titration, Karl-Fisher titration and ${ }^{1} \mathrm{H}$ NMR)

$\left[\mathrm{H}^{+}\right]: \quad \quad \quad \quad \quad .88 \mathrm{mmol}(2.20 \mathrm{M})$

$\left[\mathrm{H}_{2} \mathrm{O}\right]: \quad 3.37 \mathrm{mmol}(0.94 \mathrm{M})$

[TBP]: $\quad 7.91 \mathrm{mmol}(2.21 \mathrm{M})$

[DBP]: $\quad 5.33 \mathrm{mmol}(1.49 \mathrm{M})$

$\left[\mathrm{BuONO}_{2}\right]: 0.51 \mathrm{mmol}(0.14 \mathrm{M})$

Condensate Composition:

Aqueous Phase $(0.90 \mathrm{~g})$

$\left[\mathrm{H}^{+}\right]$: $\quad 8.60 \mathrm{mmol}(12.62 \mathrm{M})$

Organic Phase $(0.42 \mathrm{~g})$

[BuONO 2 ]: $3.35 \mathrm{mmol}(8.16 \mathrm{M})$

\section{Material Balance}

C lost from residue: $5.88 \mathrm{mmol}$ C present in Gas: $\quad 5.19 \mathrm{mmol}$

$\mathrm{N}$ lost from residue: $13.7 \mathrm{mmol}$

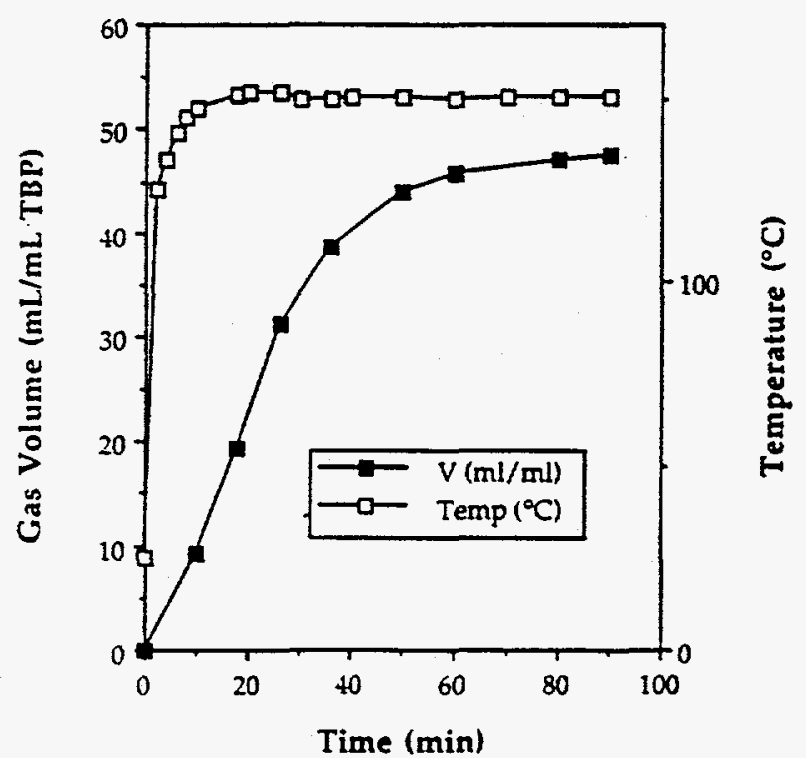

$\mathrm{N}$ present in Gas: $\quad 5.65 \mathrm{mmol}$ 
Results of Experiment No. 139 (Const. Pressure, Single Phase)

Reaction Conditions:

Bath Temperature: $\quad 130^{\circ} \mathrm{C}$

Sample Volume:

$5 \mathrm{~mL}$

Acid concentration:

$6 \mathrm{M}$

Distillation/Refluxing

$\mathrm{D}$

Atmosilhere:

Helium

Before Reaction:

Sample Weight: $5.45 \mathrm{~g}$

$\left[\mathrm{H}^{+}\right]$:

$28.75 \mathrm{mmol}(5.75 \mathrm{M})$

$\left[\mathrm{H}_{2} \mathrm{O}\right]$ :

$9.40 \mathrm{mmol}(1.88 \mathrm{M})$

[TBP]:

$13.30 \mathrm{mmol}(2.66 \mathrm{M})$

\section{After Reaction:}

Total Gas Volume: $\quad 255 \mathrm{~mL}\left(25^{\circ} \mathrm{C}, 1 \mathrm{~atm}\right)$

$\begin{array}{llllllll}\text { Gas Composition: } & \mathrm{N}_{2} & \mathrm{O}_{2} & \mathrm{CO} & \mathrm{CO}_{2} & \mathrm{NO} & \mathrm{N}_{2} \mathrm{O} & \mathrm{He}\end{array}$

$\begin{array}{llllllll}\text { total } \mathrm{mol} \mathrm{\%} & 2.3 & 0 & 9.5 & 27.8 & 44.2 & 5.9 & 1.6\end{array}$

normalized mol \% $2.6 \quad 0 \quad 10.6 \quad 31.0 \quad 49.3 \quad 6.6$

Residue Weight: $3.77 \mathrm{~g}$ (weight loss $=1.68 \mathrm{~g}$ )

Residue Composition: (by acid-base titration, Karl-Fisher titration and ${ }^{1} \mathrm{H}$ NMR)

$[\mathrm{H}+]$ :

$13.5 \mathrm{mmol}(3.58 \mathrm{M})$

$\left[\mathrm{H}_{2} \mathrm{O}\right]: \quad 3.39 \mathrm{mmol}(0.90 \mathrm{M})$

[TBP]: $\quad 7.80 \mathrm{mmol}(2.07 \mathrm{M})$

[DBP]: $\quad 5.50 \mathrm{mmol}(1.46 \mathrm{M})$

$\left[\mathrm{BuONO}_{2}\right]$ : trace

Condensate Composition:

Aqueous Phase $(0.80 \mathrm{~g})$

$\left[\mathrm{H}^{+}\right]$:

$7.03 \mathrm{mmol}(11.60 \mathrm{M})$

Organic Phase $(0.33 \mathrm{~g})$

[BuONO 2 ]: $2.63 \mathrm{mmol}(8.15 \mathrm{M})$

Material Balance

$\mathrm{C}$ loss from residue $=11.48 \mathrm{mmol}$

$\mathrm{C}$ present in gas $=\quad 4.34 \mathrm{mmol}$

$\mathrm{N}$ loss from residue $=11.09 \mathrm{mmol}$

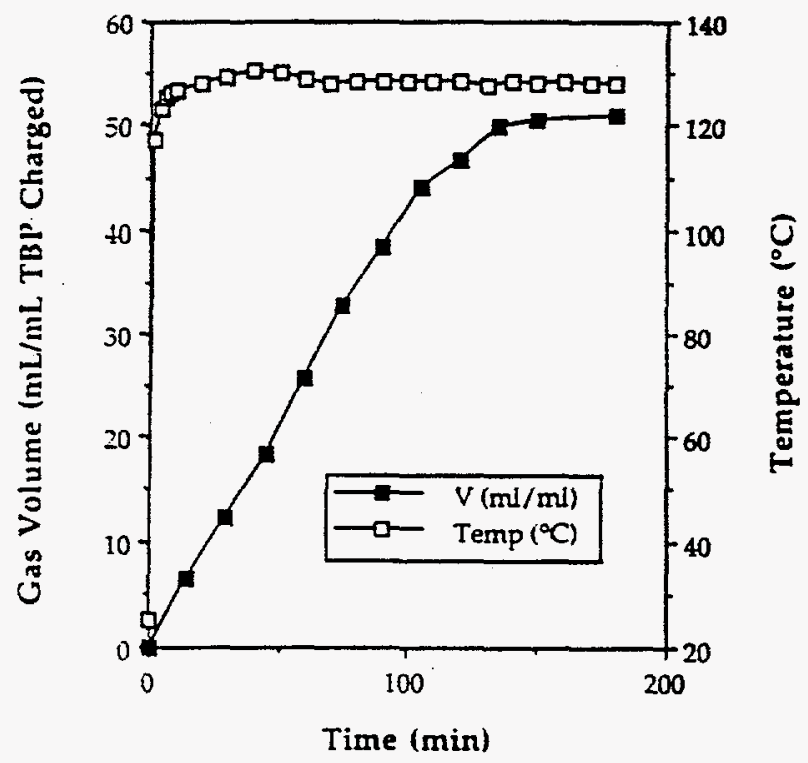

$\mathrm{N}$ present in gas $=\quad 7.07 \mathrm{mmol}$ 
WSRC-RP-95-259

Results of Experiment No. 140 (Const. Pressure, Single Phase)

Reaction Conditions:

Bath Temperature: $\quad 150^{\circ} \mathrm{C}$

Sample Volume: $\quad 5 \mathrm{~mL}$

Refluxing/Distillation D

Acid concentration:

$6 \mathrm{M}$

Atmosphere:

Helium

Before Reaction:

Sample Weight: $5.75 \mathrm{~g}$

[H+]:

$29.25 \mathrm{mmol}(5.85 \mathrm{M})$

$\left[\mathrm{H}_{2} \mathrm{O}\right]:$

$16.35 \mathrm{mmol}$ (3.27 M)

[TBP]:

$12.80 \mathrm{mmol}(2.56 \mathrm{M})$

Catalyst:

After Reaction:

Total Gas Volume: $\quad 246 \mathrm{~mL}\left(25^{\circ} \mathrm{C}, 1 \mathrm{~atm}\right)$

$\begin{array}{llllllll}\text { Gas Composition: } & \mathrm{N}_{2} & \mathrm{O}_{2} & \mathrm{CO} & \mathrm{CO}_{2} & \mathrm{NO} & \mathrm{N}_{2} \mathrm{O} & \mathrm{He}\end{array}$

$\begin{array}{llllllll}\text { total mol \% } & 3.6 & 0 & 11.9 & 28.3 & 24.4 & 5.0 & 6.9\end{array}$

$\begin{array}{lllllll}\text { normalized mol \% } & 4.9 & 0 & 16.3 & 36.7 & 33.3 & 6.8\end{array}$

Residue Weight: $3.89 \mathrm{~g}$ (weight loss $=1.86 \mathrm{~g}$ )

Residue Composition: (by acid-base titration, Karl-Fisher titration and ${ }^{1} \mathrm{H}$ NMR) $\left[\mathrm{H}^{+}\right]: \quad *$

[TBP]: $\quad 9.24 \mathrm{mmol}(2.38 \mathrm{M})$

[DBP]: $\quad 3.56 \mathrm{mmol}(0.92 \mathrm{M})$

[MBP]: trace

$\left[\mathrm{BuONO}_{2}\right]: \quad 2.12 \mathrm{mmol}(0.54 \mathrm{M})$

[PrCOOH]: trace

[EtCOOH]: trace

[MeCOOH]: $0.42 \mathrm{mmol}(0.11 \mathrm{M})$

[HCOOH] trace

Condensate Composition:

Aqueous Phase $(0.63 \mathrm{~g})$

$\left[\mathrm{H}^{+}\right]: \quad \quad 5.73 \mathrm{mmol}(12.00 \mathrm{M})$

Organic Phase $(0.89 \mathrm{~g})$

$\left[\mathrm{BuONO}_{2}\right]$ : $\quad 7.11 \mathrm{mmol}(8.22 \mathrm{M})$

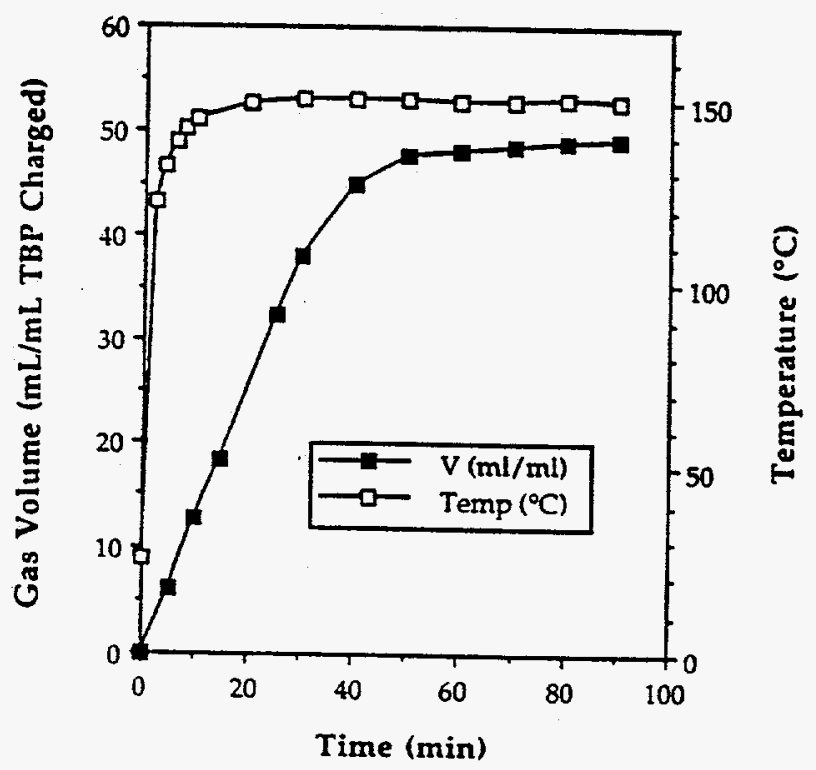

\section{Material Balance}

Not available due to the incompleteness of this set of data

* Not available because of solid residue 
WSRC-RP-95-259

Results of Experiment No. 141 (Const. Pressure, Single Phase)

Reaction Conditions:

Bath Temperature:

$180^{\circ} \mathrm{C}$

Acid concentration:

$6 \mathrm{M}$

Sample Volume:

$5 \mathrm{~mL}$

Atmosphere:

Helium

Distillation/Refluxing

Before Reaction:

Sample Weight: $5.44 \mathrm{~g}$

$\left[\mathrm{H}^{+}\right]$: $\quad 28.75 \mathrm{mmol}(5.75 \mathrm{M})$

[ $\left.\mathrm{H}_{2} \mathrm{O}\right]: \quad 9.40 \mathrm{mmol}(1.88 \mathrm{M})$

[TBP]: $\quad 13.30 \mathrm{mmol}(2.66 \mathrm{M})$

\section{After Reaction:}

Total Gas Volume: $227 \mathrm{~mL}\left(25^{\circ} \mathrm{C}, 1 \mathrm{~atm}\right)$

Gas Composition: $\mathrm{N}_{2} \quad \mathrm{O}_{2}$

$\mathrm{CO}$

$\mathrm{CO}_{2} \mathrm{NO}$

$\mathrm{N}_{2} \mathrm{O} \mathrm{He}$

total $\mathrm{mol} \mathrm{\%}$

$3.0 \quad 0$

9.3

23.

normalized mol \% $4.3 \quad 0$

$\begin{array}{llll}13.4 & 33.2 & 43.1 & 6.0\end{array}$

Residue Weight: $3.37 \mathrm{~g}$ (weight loss $=2.07 \mathrm{~g}$ )

Residue Composition: (by acid-base titration, Karl-Fisher titration and ${ }^{1} \mathrm{H}$ NMR)

$\left[\mathrm{H}^{+}\right]$: $7.01 \mathrm{mmol}(2.08 \mathrm{M})$

$\left[\mathrm{H}_{2} \mathrm{O}\right]$ : $1.08 \mathrm{mmol}(0.32 \mathrm{M})$

[TBP]: $8.01 \mathrm{mmol}(2.38 \mathrm{M})$

[DBP]: $4.34 \mathrm{mmol}(1.29 \mathrm{M})$

[MBP]: $0.95 \mathrm{mmol}(0.28 \mathrm{M})$

[BuONO${ }_{2}$ ]: $0.24 \mathrm{mmol}(0.07 \mathrm{M})$

[PrCOOH]: trace

[EtCOOH]: $\quad 0.16 \mathrm{mmol}(0.05 \mathrm{M})$

[MeCOOH]: $0.11 \mathrm{mmol}(0.03 \mathrm{M})$

[HCOOH]: $\quad 0.33 \mathrm{mmol}(0.10 \mathrm{M})$

Condensate Composition:

Aqueous Phase $(1.80 \mathrm{~g})$

$\left[\mathrm{H}^{+}\right]$:

$17.19 \mathrm{mmol}(12.60 \mathrm{M})$

Organic Phase $(0.33 \mathrm{~g})$

[BuONO 2$]: \quad 2.63 \mathrm{mmol}(8.20 \mathrm{M})$

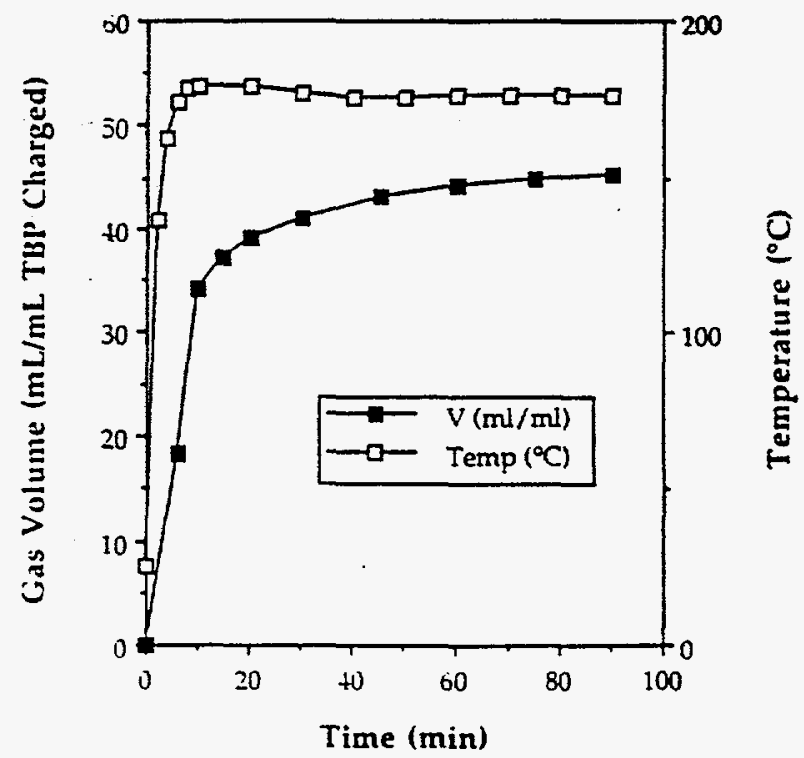

\section{Material Balance:}

$C$ loss from residue $=12.45 \mathrm{mmol}$

$C$ present in gas $=4.33 \mathrm{mmol}$

$\mathrm{N}$ loss from residue $=8.52 \mathrm{mmol}$

$\mathrm{N}$ present in gas $=5.92 \mathrm{mmol}$ 
WSRC-RP-95-259

Results of Experiment No. 142 (Const. Volume, Single Phase)

Reaction Conditions:

Bath Temperature: $\quad 130^{\circ} \mathrm{C}$

Sample Volume: $\quad 5 \mathrm{~mL}$

Acid concentration: $\quad 6 \mathrm{M}$

Before Reaction:

Sample Weight: $5.42 \mathrm{~g}$

$\left[\mathrm{H}^{+}\right]$:

$29.25 \mathrm{mmol}(5.85 \mathrm{M})$

$\left[\mathrm{H}_{2} \mathrm{O}\right]$ :

$9.40 \mathrm{mmol}(1.88 \mathrm{M})$

[TBP]:

$13.30 \mathrm{mmol}(2.66 \mathrm{M})$

\section{After Reaction:}

Total Gas Pressure: $\quad 8.7$ atms $\left(25^{\circ} \mathrm{C}, 58 \mathrm{~mL}\right.$ volume)

$\begin{array}{llllllll}\text { Gas Composition: } & \mathrm{N}_{2} & \mathrm{O}_{2} & \mathrm{CO} & \mathrm{CO}_{2} & \mathrm{NO} & \mathrm{N}_{2} \mathrm{O} & \mathrm{He}\end{array}$

$\begin{array}{llllllll}\text { total mol \% } & 7.7 & 0 & 20.3 & 32.1 & 15.7 & 7.0 & 9.0\end{array}$

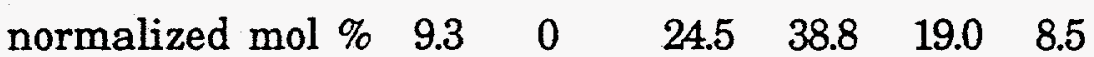

Residue Weight: $4.67 \mathrm{~g}$ (weight loss $=0.75 \mathrm{~g}$ )

Residue Composition: (by acid-base titration, Karl-Fisher titration and ${ }^{1} \mathrm{H}$ NMR)

Organic Phase (4.41 g)

$\left[\mathrm{H}^{+}\right]$: $\quad 16.35 \mathrm{mmol}(3.50 \mathrm{M})$

$\left[\mathrm{H}_{2} \mathrm{O}\right]: \quad \quad 13.03 \mathrm{mmol}(2.79 \mathrm{M})$

[TBP]: $\quad 7.88 \mathrm{mmol}(1.56 \mathrm{M})$

[DBP]: $\quad 5.42 \mathrm{mmol}(1.38 \mathrm{M})$

[MBP]: trace

[BuONO 2$]: \quad 1.66 \mathrm{mmol}(0.70 \mathrm{M})$

[PrCOOH]: trace

[EtCOOH]: $2.25 \mathrm{mmol}(0.48 \mathrm{M})$

[MeCOOH]: $0.79 \mathrm{mmol}(0.17 \mathrm{M})$

[HCOOH]: trace

Aqueous Phase (0.26 g)

$\left[\mathrm{H}^{+}\right]: \quad \quad 1.19 \mathrm{mmol}(5.50 \mathrm{M})$

$\left[\mathrm{BuONO}{ }_{2}\right]:$ trace

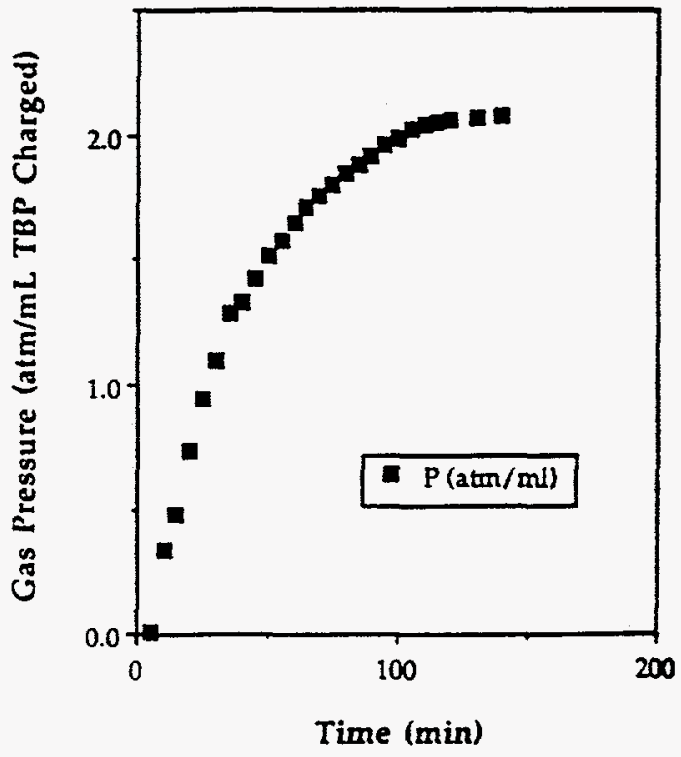

Carbon present in gas $=13.07 \mathrm{mmol}$ Nitrogen present in gas $=11.27 \mathrm{mmol}$ 


\section{Results of Experiment No. 143 (Const. Volume, Single Phase).}

Reaction Conditions:

Bath Temperature: $\quad 180^{\circ} \mathrm{C}$

Sample Volume: $\quad 5 \mathrm{~mL}$

Acid concentration: $\quad 6 \mathrm{M}$

Before Reaction:

Sample Weight: $5.46 \mathrm{~g}$

$\left[\mathrm{H}^{+}\right]$: $28.75 \mathrm{mmol}(5.75 \mathrm{M})$

$\left[\mathrm{H}_{2} \mathrm{O}\right]$ : $9.40 \mathrm{mmol}(1.88 \mathrm{M})$

[TBP]: $13.30 \mathrm{mmol}(2.66 \mathrm{M})$

Atmosphere:

Helium

\section{After Reaction:}

Total Pressure:

10.8 atms $\left(25^{\circ} \mathrm{C}, 58 \mathrm{~mL}\right.$ volume $)$

Gas Composition:

$\begin{array}{llllllll}\mathrm{N}_{2} & \mathrm{O}_{2} & \mathrm{CO} & \mathrm{CO}_{2} & \mathrm{NO} & \mathrm{N}_{2} \mathrm{O} & \mathrm{He}\end{array}$

total $\mathrm{mol} \mathrm{\%}$

$\begin{array}{lllllll}8.3 & 0 & 26.0 & 35.0 & 5.3 & 13.2 & 6.0\end{array}$

normalized mol \% $9.5 \quad 0 \quad 39.6 \quad 39.9 \quad 6.0 \quad 15.0$

Residue Weight: $4.29 \mathrm{~g}$ (weight loss $=1.17 \mathrm{~g}$ )

Residue Composition: (by acid-base titration, Karl-Fisher titration and ${ }^{1} \mathrm{H}$ NMR) Organic Phase

$\left[\mathrm{H}^{+}\right]$:

$\left[\mathrm{H}_{2} \mathrm{O}\right]$ :

[TBP]:

$16.04 \mathrm{mmol}(3.74 \mathrm{M})$

[DBP]:

$22.61 \mathrm{mmol}(5.27 \mathrm{M})$

[MBP]:

$5.41 \mathrm{mmol}(1.26 \mathrm{M})$

$5.54 \mathrm{mmol}(1.29 \mathrm{M})$

$\left[\mathrm{BuONO}{ }_{2}\right]: \quad 1.08 \mathrm{mmol}(0.25 \mathrm{M})$

[Bu-OH]: $\quad 1.02 \mathrm{mmol}(0.24 \mathrm{M})$

[PrCOOH]: Trace

[EtCOOH]: $\quad 1.42 \mathrm{mmol}(0.33 \mathrm{M})$

[MeCOOH]: $1.29 \mathrm{mmol}(0.30 \mathrm{M})$

[HCOOH]: $\quad 0.34 \mathrm{mmol}(0.08 \mathrm{M})$

Aqueous Phase $(0.17 \mathrm{~g})$

$\left[\mathrm{H}^{+}\right]$:

$0.64 \mathrm{mmol}(4.52 \mathrm{M})$

[BuONO$\left.{ }_{2}\right]$ : trace

Material Balance

Carbon loss from residue $=25.38 \mathrm{mmol}$

Nitrogen loss from residue $=24.28 \mathrm{mmol}$

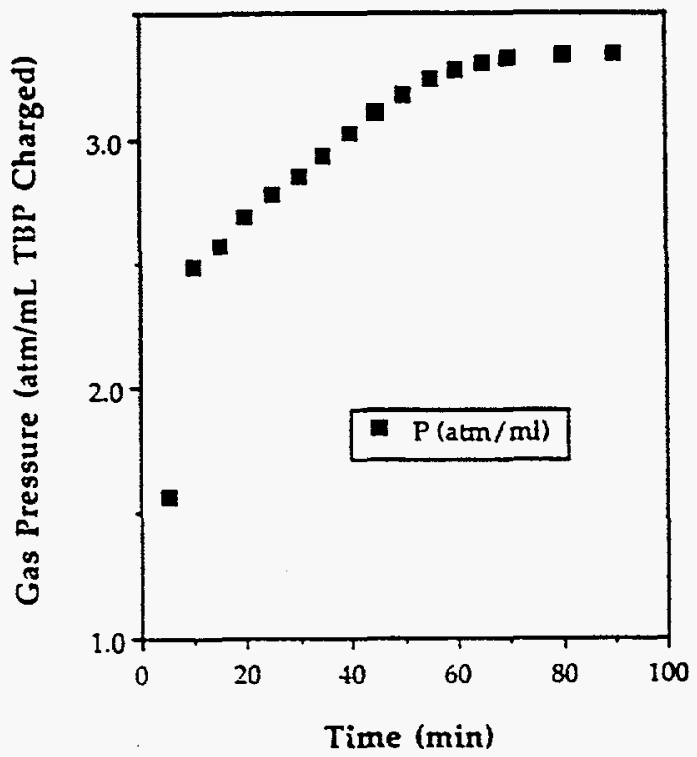

Carbon present in gas $=17.81 \mathrm{mmol}$ Nitrogen present in gas $=14.10 \mathrm{mmol}$ 


\section{Results for Experiment No. 144 (Const. Pressure, Single Phase)}

Reaction Conditions:

Bath Temperature: $\quad 150^{\circ} \mathrm{C}$

Sample Volume: $\quad 5.0 \mathrm{~mL}$

Acid concentration:

$6 \mathrm{M}$

Distillation/Refluxing $R$

Atmosphere:

Helium

Before Reaction:

Sample Weight: $5.43 \mathrm{~g}$

$[\mathrm{H}+]$ :

$29.0 \mathrm{mmol}(5.80 \mathrm{M})$

$\left[\mathrm{H}_{2} \mathrm{O}\right]$ :

$9.40 \mathrm{mmol}(1.88 \mathrm{M})$

[TBP]:

$13.3 \mathrm{mmol}(2.66 \mathrm{M})$

\section{After Reaction:}

Total Gas Volume: $\quad 312 \mathrm{~mL}\left(25^{\circ} \mathrm{C}, 1 \mathrm{~atm}\right)$

$\begin{array}{llllllll}\text { Gas Composition: } & \mathrm{N}_{2} & \mathrm{O}_{2} & \mathrm{CO} & \mathrm{CO}_{2} & \mathrm{NO} & \mathrm{N}_{2} \mathrm{O} & \mathrm{He}\end{array}$

$\begin{array}{llllllll}\text { total } \mathrm{mol} \mathrm{\%} & 2.6 & 0 & 7.0 & 16.6 & 40.7 & 3.5 & 21.8\end{array}$

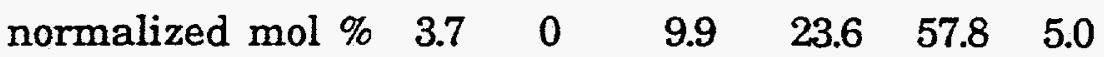

Residue Weight: $\quad 4.83 \mathrm{~g}$ (loss $=0.60 \mathrm{~g}$ )

Residue Composition: (by acid-base titration, Karl-Fisher titration and ${ }^{1} \mathrm{H}$ NMR) Organic Phase (4.52 g)

$\left[\mathrm{H}^{+}\right]$:

$18.13 \mathrm{mmol}(4.01 \mathrm{M})$

$\left[\mathrm{H}_{2} \mathrm{O}\right]: \quad 10.94 \mathrm{mmol}(2.42 \mathrm{M})$

[TBP]: $\quad 8.39 \mathrm{mmol}(1.86 \mathrm{M})$

[DBP]: $\quad 4.91 \mathrm{mmol}(1.09 \mathrm{M})$

[MBP]: Trace

[BuONO 2 ]: $2.62 \mathrm{mmol}(0.58 \mathrm{M})$

[PrCOOH] trace

[EtCOOH]: $0.74 \mathrm{mmol}(0.16 \mathrm{M})$

[MeCOOH]: $0.65 \mathrm{mmol}(0.14 \mathrm{M})$

[HCOOH]: trace

Aqueous Phase $(0.31 \mathrm{~g})$

$\left[\mathrm{H}^{+}\right]$: $\quad 2.05 \mathrm{mmol}(8.26 \mathrm{M})$

$\left[\mathrm{BuONO}_{2}\right]$ : trace

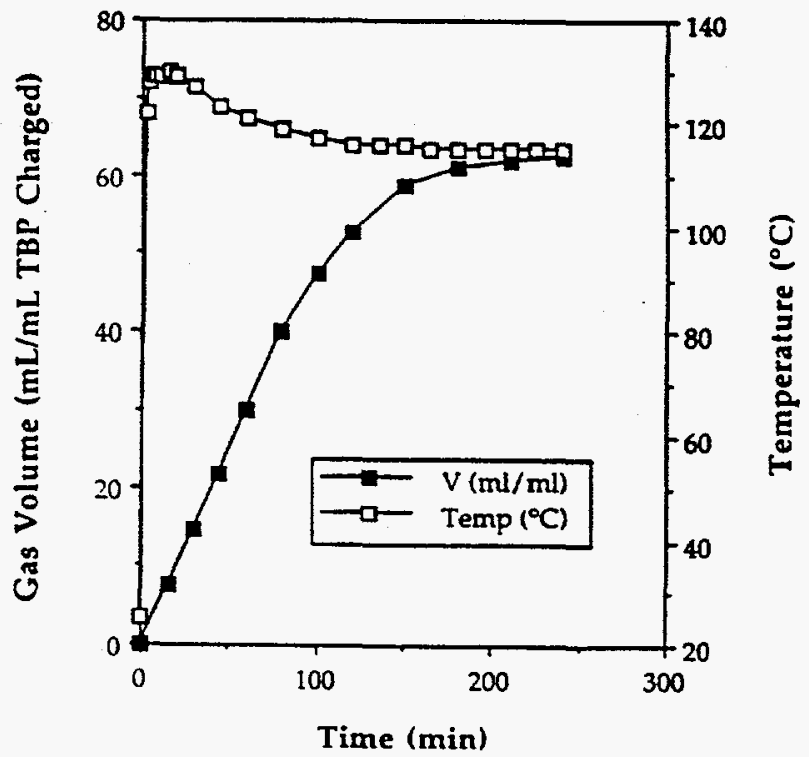

\section{Material Balance}

Carbon loss in residue $=5.64 \mathrm{mmol}$

Nitrogen loss in residue $=12.50 \mathrm{mmol}$

Carbon present in gas $=4.28 \mathrm{mmol}$ Nitrogen present in gas $=9.60 \mathrm{mmol}$ 
WSRC-RP-95-259

\section{Results of Experiment No. 145 (Const. Pressure, Single Phase)}

\section{Reaction Conditions:}

Bath Temperature: $\quad 130^{\circ} \mathrm{C}$

Sample Volume: $\quad 5 \mathrm{~mL}$

Distillation/Refluxing $R$

Atmosphere:

$6 \mathrm{M}$

Before Reaction:

Sample Weight: $5.41 \mathrm{~g}$

$\left[\mathrm{H}^{+}\right]$:

$28.75 \mathrm{mmol}(5.75 \mathrm{M})$

$\left[\mathrm{H}_{2} \mathrm{O}\right]:$

$9.40 \mathrm{mmol}(1.88 \mathrm{M})$

[TBP]:

$13.30 \mathrm{mmol}(2.66 \mathrm{M})$

\section{After Reaction:}

Total Gas Volume: $\quad 351 \mathrm{~mL}\left(25^{\circ} \mathrm{C}, 1 \mathrm{~atm}\right)$

$\begin{array}{llllllll}\text { Gas Composition: } & \mathrm{N}_{2} & \mathrm{O}_{2} & \mathrm{CO} & \mathrm{CO}_{2} & \mathrm{NO} & \mathrm{N}_{2} \mathrm{O} & \mathrm{He}\end{array}$ $\begin{array}{llllllll}\text { total mol \% } & 1.4 & 0 & 6.7 & 19.4 & 47.0 & 3.9 & 15.9\end{array}$

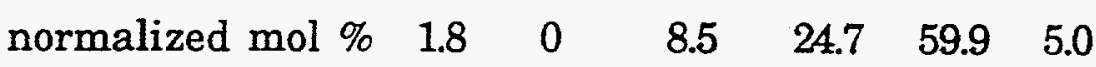

Residue Weight: $4.74 \mathrm{~g}$ (weight loss $=0.67 \mathrm{~g}$ )

Residue Composition: (by acid-base titration, Karl-Fisher titration and ${ }^{1} \mathrm{H}$ NMR) $\left[\mathrm{H}^{+}\right]$:

$19.01 \mathrm{mmol}(4.01 \mathrm{M})$

$\left[\mathrm{H}_{2} \mathrm{O}\right]:$

$6.73 \mathrm{mmol}(1.42 \mathrm{M})$

[TBP]: $\quad 8.30 \mathrm{mmol}(1.75 \mathrm{M})$

[DBP]: $\quad 5.00 \mathrm{mmol}(1.05 \mathrm{M})$

[MBP]: Trace

[BuONO${ }_{2}$ ]: $2.42 \mathrm{mmol}(0.51 \mathrm{M})$

[PrCOOH]: trace

[EtCOOH]: $0.61 \mathrm{mmol}(0.13 \mathrm{M})$

[MeCOOH]: $0.61 \mathrm{mmol}(0.13 \mathrm{M})$

[HCOOH]: trace

\section{Material Balance}

C loss from residue: $7.27 \mathrm{mmol}$

$\mathrm{C}$ present in gas:

$4.77 \mathrm{mmol}$

$\mathrm{N}$ loss from residue: $13.54 \mathrm{mmol}$

$\mathrm{N}$ present in gas: $\quad 10.55 \mathrm{mmol}$

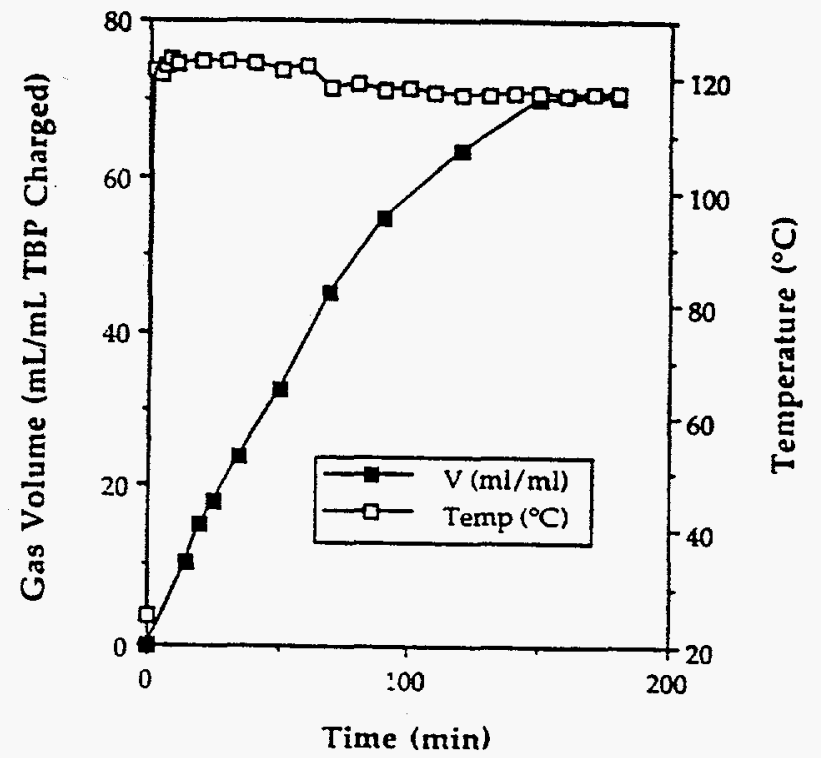




\section{Results of Experiment No. 146 (Const. Pressure, Single Phase)}

\section{Reaction Conditions:}

Bath Temperature: $\quad 180^{\circ} \mathrm{C}$

Sample Volume: $\quad 5 \mathrm{~mL}$

Acid concentration: $\quad 6 \mathrm{M}$

Refluxing/Distillation $R$

Atmosphere:

Helium

Before Reaction:

Sample Weight: $5.43 \mathrm{~g}$

$\left[\mathrm{H}^{+}\right]$:

$28.75 \mathrm{mmol}(5.75 \mathrm{M})$

$\left[\mathrm{H}_{2} \mathrm{O}\right]$ :

$9.40 \mathrm{mmol}(1.88 \mathrm{M})$

[TBP]:

$13.30 \mathrm{mmol}(2.66 \mathrm{M})$

After Reaction:

Total Gas Volume: $\quad 432 \mathrm{ml}\left(25^{\circ} \mathrm{C}, 1 \mathrm{~atm}\right)$

$\begin{array}{llllllll}\text { Gas Composition: } & \mathrm{N}_{2} & \mathrm{O}_{2} & \mathrm{CO} & \mathrm{CO}_{2} & \mathrm{NO} & \mathrm{N}_{2} \mathrm{O} & \mathrm{He}\end{array}$ $\begin{array}{llllllll}\text { total } \mathrm{mol} \% & 1.0 & 0 & 4.1 & 21.1 & 47.3 & 5.2 & 14.9\end{array}$

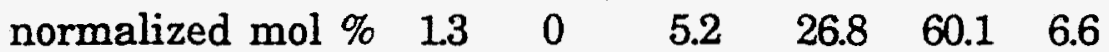

Residue Weight: $4.66 \mathrm{~g}$ (weight loss $=0.77 \mathrm{~g}$ )

Residue Composition: (by acid-base titration, Karl-Fisher titration and $1 \mathrm{H}$ NMR) Organic Phase $(4.01 \mathrm{~g})$

$\left[\mathrm{H}^{+}\right]$:

$16.56 \mathrm{mmol}(4.13 \mathrm{M})$

$\left[\mathrm{H}_{2} \mathrm{O}\right]: \quad 12.6 \mathrm{mmol}(3.14 \mathrm{M})$

[TBP]: $\quad 7.24 \mathrm{mmol}(1.81 \mathrm{M})$

[DBP]: $\quad 5.43 \mathrm{mmol}(1.35 \mathrm{M})$

[MBP]: $\quad 0.63 \mathrm{mmol}(0.16 \mathrm{M})$

$\left[\mathrm{BuONO}_{2}\right]: \quad 3.14 \mathrm{mmol}(0.78 \mathrm{M})$

[PrCOOH]: trace

[EtCOOH]: $1.13 \mathrm{mmol}(0.28 \mathrm{M})$

[MeCOOH]: $0.64 \mathrm{mmol}(0.16 \mathrm{M})$

[HCOOH]: trace

Aqueous Phase $(0.65 \mathrm{~g})$

$\left[\mathrm{H}^{+}\right]$:

$4.26 \mathrm{mmol}(7.87 \mathrm{M})$

$\left[\mathrm{BuONO}_{2}\right]$ : trace

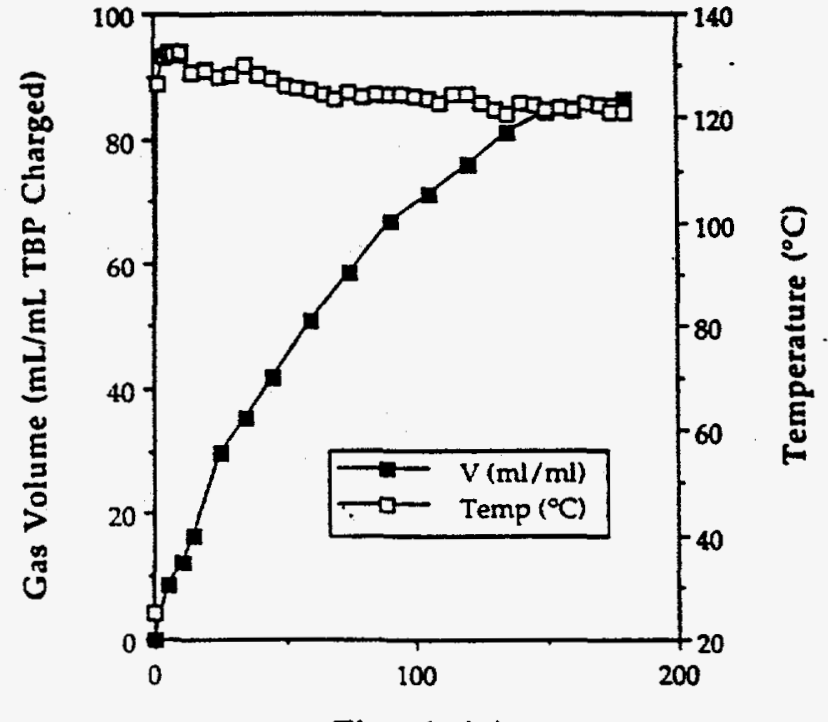

Time (min)

\section{Material Balance}

Carbon loss from residue $=9.53 \mathrm{mmol}$

Carbon present in gas $=5.65 \mathrm{mmol}$

Nitrogen loss from residue $=13.25 \mathrm{mmol}$ 


\section{Results of Experiment No. 147A (Const. Pressure, Single Phase, No Water)}

Reaction Conditions:

Bath Temperature: $\quad 150^{\circ} \mathrm{C}$

Sample Volume: $\quad 5 \mathrm{~mL}$

Refluxing/Distillation D

Acid concentration: $\quad 3 \mathrm{M}$

Before Reaction:

Sample Weight: $5.13 \mathrm{~g}$

$\left[\mathrm{H}^{+}\right]$:

$15.75 \mathrm{mmol}(3.15 \mathrm{M})$

Atmosphere:

Helium

[TBP]: $\quad 15.85 \mathrm{mmol}(3.17 \mathrm{M})$

$\left[\mathrm{H}_{2} \mathrm{O}\right]:$

$0 \mathrm{mmol}$.

\section{After Reaction:}

Total Gas Volume: $\quad 289 \mathrm{~mL}\left(25^{\circ} \mathrm{C}, 1 \mathrm{~atm}\right)$

Gas Composition: $\quad \mathrm{N}_{2} \quad \mathrm{O}_{2} \quad \mathrm{CO} \quad \mathrm{CO}_{2} \quad \mathrm{NO} \quad \mathrm{N}_{2} \mathrm{O} \quad \mathrm{He}$

$\begin{array}{llllllll}\text { total mol \% } & 5.0 & 0 & 8.4 & 23.5 & 34.1 & 5.7 & 10.0 \\ \text { normalized mol \% } & 6.5 & 0 & 11.0 & 30.6 & 44.5 & 7.4 & \end{array}$

Residue Weight: $4.17 \mathrm{~g}$ (weight loss $=0.97 \mathrm{~g}$ )

Residue Composition: (by acid-base titration, Karl-Fisher titration and ${ }^{1} \mathrm{H}$ NMR)

$\left[\mathrm{H}^{+}\right]$: $\quad \quad \quad 7.38 \mathrm{mmol}(1.77 \mathrm{M})$

$\left[\mathrm{H}_{2} \mathrm{O}\right]: \quad 2.63 \mathrm{mmol}(0.63 \mathrm{M})$

[TBP]: $\quad 11.57 \mathrm{mmol}(2.77 \mathrm{M})$

[DBP]: $\quad 4.18 \mathrm{mmol}(1.00 \mathrm{M})$

[MeCOOH]: $0.13 \mathrm{mmol}(0.03 \mathrm{M})$

Condensate Composition:

Aqueous Phase $(0.20 \mathrm{~g})$

$\left[\mathrm{H}^{+}\right]$:

$1.45 \mathrm{mmol}(9.44 \mathrm{M})$

Organic Phase $(0.32 \mathrm{~g})$

$\left[\mathrm{BuONO}_{2}\right] \quad 2.55 \mathrm{mmol}(8.61 \mathrm{M})$

Material Balance

C loss from residue: $6.26 \mathrm{mmol}$

C present in gas: $\quad 3.89 \mathrm{mmol}$

$\mathrm{N}$ loss from residue: $8.64 \mathrm{mmol}$

$\mathrm{N}$ present in gas: $\quad 8.55 \mathrm{mmol}$

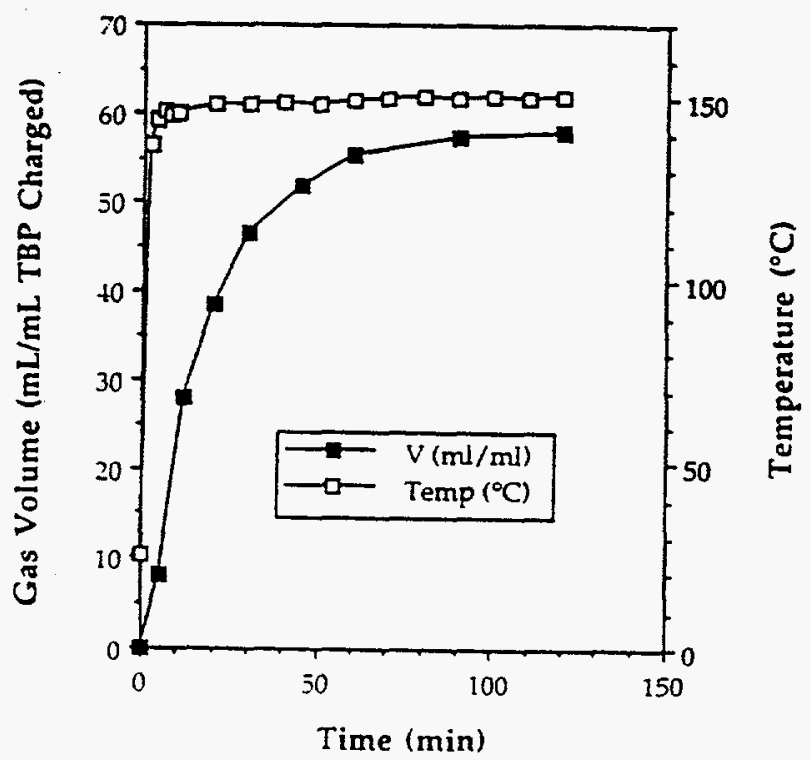


Results of Experiment No. 147B (Const. Volume, Single Phase, No Water)

\section{Reaction Conditions:}

Bath Temperature:

Sample Volume:

Before Reaction:

Sample Weight: $5.15 \mathrm{~g}$

$\left[\mathrm{H}^{+}\right]$:

$\left[\mathrm{H}_{2} \mathrm{O}\right]$ :

[TBP]:

$$
\begin{aligned}
& 150^{\circ} \mathrm{C} \\
& 5 \mathrm{ml}
\end{aligned}
$$

$15.75 \mathrm{mmol}(3.15 \mathrm{M})$

$0 \mathrm{mmol}$

$15.85 \mathrm{mmol}(3.17 \mathrm{M})$
Acid Concentration:

Atmosphere:
$3 \mathbf{M}$

Helium

\section{After Reaction:}

Total Gas Pressure: $\quad 5.4 \mathrm{~atm}\left(25^{\circ} \mathrm{C}, 58 \mathrm{~mL}\right.$ volume)

$\begin{array}{llllllll}\text { Gas Composition: } & \mathrm{N}_{2} & \mathrm{O}_{2} & \mathrm{CO} & \mathrm{CO}_{2} & \mathrm{NO} & \mathrm{N}_{2} \mathrm{O} & \mathrm{He}\end{array}$ $\begin{array}{llllllll}\text { total mol \% } & 23.4 & 0.7 & 20.1 & 24.4 & 0 & 9.0 & 14.0\end{array}$

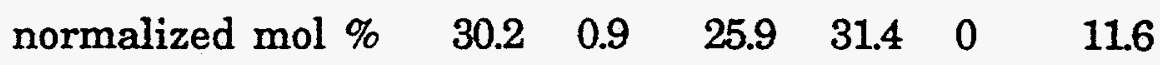

Residue Weight: $4.68 \mathrm{~g}$ (weight loss $=0.47 \mathrm{~g}$ )

Residue Composition: (by acid-base titration, Karl-Fisher titration and ${ }^{1} \mathrm{H}$ NMR) $\left[\mathrm{H}^{+}\right]$: $\quad 8.85 \mathrm{mmol}(1.89 \mathrm{M})$

$\left[\mathrm{H}_{2} \mathrm{O}\right]: \quad 9.69 \mathrm{mmol}(2.07 \mathrm{M})$

[TBP]: $\quad 11.20 \mathrm{mmol}(2.39 \mathrm{M})$

[DBP]: $\quad 4.55 \mathrm{mmol}(0.97 \mathrm{M})$

$\left[\mathrm{BuONO}{ }_{2}\right]: \quad 1.86 \mathrm{mmol}(0.40 \mathrm{M})$

[PrCOOH]: $0.65 \mathrm{mmol}(0.14 \mathrm{M})$

[EtCOOH]: $0.48 \mathrm{mmol}(0.10 \mathrm{M})$

[MeCOOH]: $1.01 \mathrm{mmol}(0.22 \mathrm{M})$

[HCOOH]: $0.16 \mathrm{mmol}(0.03 \mathrm{M})$

Material Balance

C loss from residue: $4.54 \mathrm{mmol}$

C present in gas: $\quad 7.35 \mathrm{mmol}$

$\mathrm{N}$ loss from residue: $11.89 \mathrm{mmol}$

$\mathrm{N}$ present in gas: $\quad 10.72 \mathrm{mmol}$

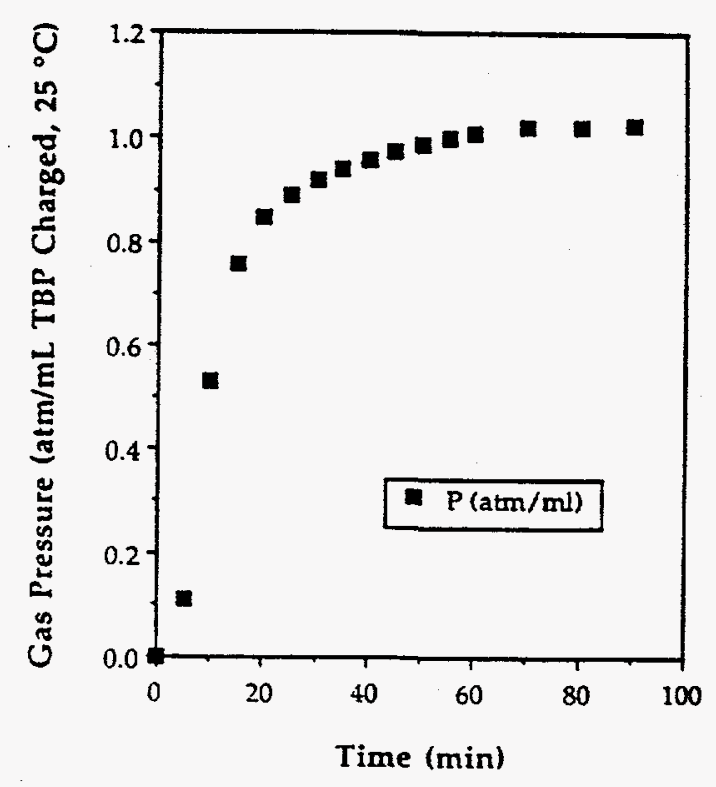




\section{Results of Experiment No. 148A (Const. Pressure, Single Phase)}

Bath Temperature:

Sample Volume:

Refluxing/Distillation

\section{Before Reaction:}

Sample Weight: $5.49 \mathrm{~g}$

$\left[\mathrm{H}^{+}\right]$ $28.75 \mathrm{mmol}(5.75 \mathrm{M})$

[TBP]: $13.25 \mathrm{mmol}(2.65 \mathrm{M})$
Acid concentration:

Atmosphere:

$6 \mathrm{M}$

$5 \mathrm{~mL}$

$\mathrm{D}$

Helium

\section{After Reaction:}

Total Gas Volume: $\quad 243 \mathrm{~mL}\left(25^{\circ} \mathrm{C}, 1 \mathrm{~atm}\right)$

$\begin{array}{lllllllll}\text { Gas Composition: } & \mathrm{N}_{2} & \mathrm{O}_{2} & \mathrm{CO} & \mathrm{CO}_{2} & \mathrm{NO} & \mathrm{N}_{2} \mathrm{O} & \mathrm{He}\end{array}$

$\begin{array}{lllllllll}\text { total mol \% } & 1.5 & 0 & 9.0 & 18.6 & 18.6 & 4.1 & 27.4\end{array}$

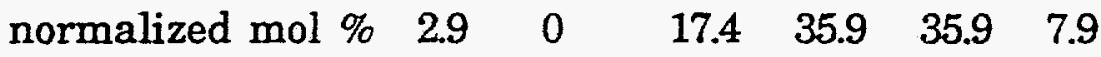

Sample Weight: $3.65 \mathrm{~g}$ (weight loss $=1.84 \mathrm{~g}$ )

Residue Composition: (by acid-base titration, Karl-Fisher titration and ${ }^{1} \mathrm{H}$ NMR)

$\left[\mathrm{H}^{+}\right]$: $\quad 7.98 \mathrm{mmol}(2.19 \mathrm{M})$

$\left[\mathrm{H}_{2} \mathrm{O}\right]: \quad 3.17 \mathrm{mmol}(0.87 \mathrm{M})$

[TBP]: $\quad 8.36 \mathrm{mmol}(2.29 \mathrm{M})$

[DBP]: $\quad 4.89 \mathrm{mmol}(1.34 \mathrm{M})$

[BuONO 2$]: \quad 0.72 \mathrm{mmol}(0.14 \mathrm{M})$

[MeCOOH]: $0.24 \mathrm{mmol}(0.07 \mathrm{M})$

Condensate Composition:

Aqueous Phase $(0.98 \mathrm{~g})$

$\left[\mathrm{H}^{+}\right]$:

$9.21 \mathrm{mmol}(12.41 \mathrm{M})$

Organic Phase $(0.22 \mathrm{~g})$

[BuONO 2$]: \quad 1.76 \mathrm{mmol}(8.23 \mathrm{M})$

Material Balance

$C$ loss from residue: $9.16 \mathrm{mmol}$

$\mathrm{C}$ present in Gas: $\quad 5.30 \mathrm{mmol}$

$\mathrm{N}$ loss from residue: $14.21 \mathrm{mmol}$

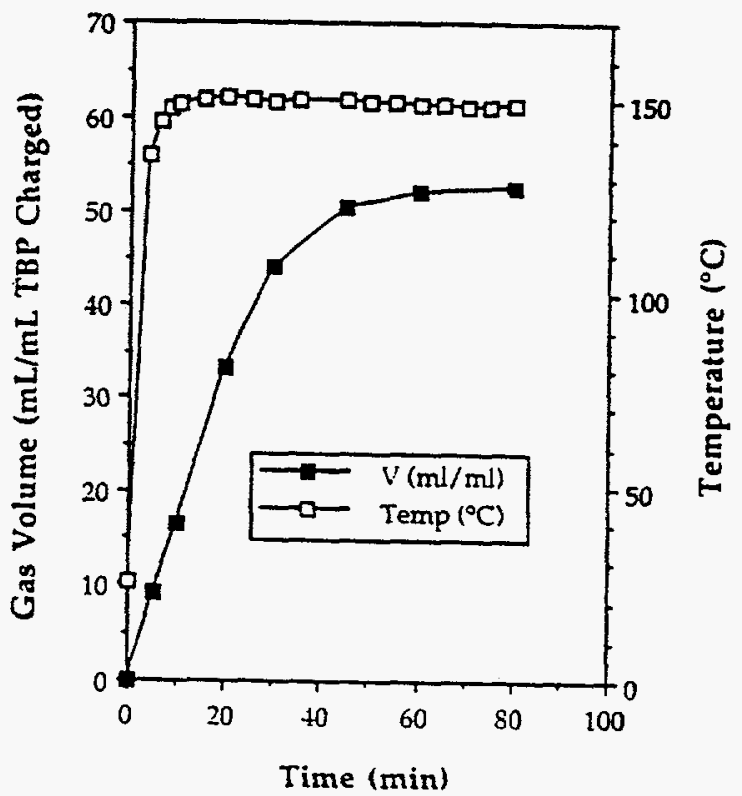

$\mathrm{N}$ present in Gas: $\quad 5.72 \mathrm{mmol}$ 


\section{Results of Experiment No. 148B (Const.Pressure, Single Phase)}

Bath Temperature: $\quad 150^{\circ} \mathrm{CAcid}$ concentration: $\quad 3 \mathrm{M}$

Sample Volume: 5 mLAtmosphere: Helium

Refluxing/Distillation D

Before Reaction:

Sample Weight: $\quad 5.13 \mathrm{~g}$

$\left[\mathrm{H}^{+}\right]$:

$15.00 \mathrm{mmol}(3.00 \mathrm{M})$

$\left[\mathrm{H}_{2} \mathrm{O}\right]:$

$7.40 \mathrm{mmol}(1.48 \mathrm{M})$

[TBP]:

$14.20 \mathrm{mmol}(2.84 \mathrm{M})$

\section{After Reaction:}

Total Gas Volume: $\quad 185 \mathrm{~mL}\left(25^{\circ} \mathrm{C}\right.$, 1atm)

$\begin{array}{lllllllll}\text { Gas Composition: } & \mathrm{N}_{2} & \mathrm{O}_{2} & \mathrm{CO} & \mathrm{CO}_{2} & \mathrm{NO} & \mathrm{N}_{2} \mathrm{O} & \mathrm{He}\end{array}$

$\begin{array}{llllllll}\text { total mol \% } & 2.1 & 0 & 11.6 & 15.6 & 15.8 & 4.1 & 43.2 \\ \text { normalized mol \% } & 4.3 & 0 & 23.6 & 31.7 & 32.1 & 8.3 & \end{array}$

Sample Weight: $3.92 \mathrm{~g}$ (weight loss $=1.21 \mathrm{~g}$ )

Residue Composition: (by acid-base titration, Karl-Fisher titration and ${ }^{1} \mathrm{H}$ NMR)

$\left[\mathrm{H}^{+}\right]$: $\quad 8.82 \mathrm{mmol}(2.25 \mathrm{M})$

$\left[\mathrm{H}_{2} \mathrm{O}\right]: \quad 3.18 \mathrm{mmol}(0.81 \mathrm{M})$

[TBP]: $\quad 9.57 \mathrm{mmol}(2.44 \mathrm{M})$

[DBP]: $\quad$ 4.63. $\mathrm{mmol}(1.18 \mathrm{M})$

[BuONO 2 ]: $0.54 \mathrm{mmol}(0.14 \mathrm{M})$

[MeCOOH]: $0.34 \mathrm{mmol}(0.09 \mathrm{M})$

Condensate Composition:

Aqueous Phase $(0.29 \mathrm{~g})$

$\left[\mathrm{H}^{+}\right]: \quad \quad 1.60 \mathrm{mmol}(5.78 \mathrm{M})$

Organic Phase (0.32 g)

[BuONO${ }_{2}$ ]: $2.25 \mathrm{mmol}(8.20 \mathrm{M})$

\section{Material Balance}

C lost from residue: $6.68 \mathrm{mmol}$

C present in Gas: $\quad 4.19 \mathrm{mmol}$

C lost from residue: $\quad 6.76 \mathrm{mmol}$

C present in Gas: $\quad 4.34 \mathrm{mmol}$

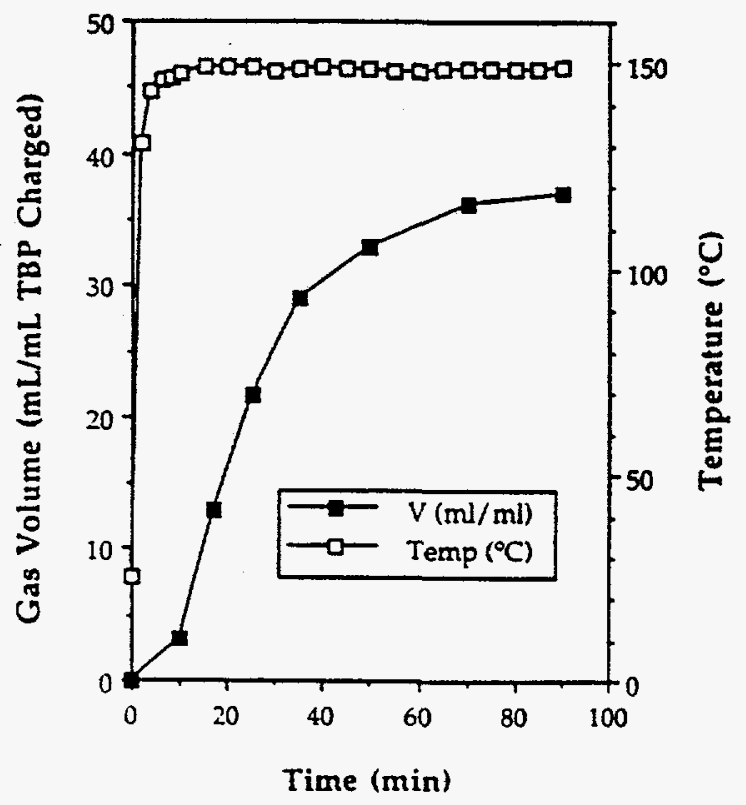


WSRC-RP-95-259

Results of Experiment No. 148C (Const. Pressure, Single Phase)

Reaction Conditions:

Bath Temperature: $\quad 150^{\circ} \mathrm{C}$

Sample Volume: $\quad 5 \mathrm{~mL}$

Refluxing/Distillation $D$

D Catalyst:

Acia ec :centration: $\quad 6 \mathrm{M}$

Before Reaction:

Sample Weight: $6.04 \mathrm{~g}$

$\left[\mathrm{H}^{+}\right]$: $\quad 29.25 \mathrm{mmol}(5.85 \mathrm{M})$

$\left[\mathrm{H}_{2} \mathrm{O}\right]: \quad 16.35 \mathrm{mmol}(3.27 \mathrm{M})$

[TBP]: $\quad 12.80 \mathrm{mmol}(2.56 \mathrm{M})$

After Reaction:

Total Gas Volume: $\quad 228 \mathrm{~mL}\left(25^{\circ} \mathrm{C}, 1 \mathrm{~atm}\right)$

$\begin{array}{lllllllll}\text { Gas Composition: } & \mathrm{N}_{2} & \mathrm{O}_{2} & \mathrm{CO} & \mathrm{CO}_{2} & \mathrm{NO} & \mathrm{N}_{2} \mathrm{O} & \mathrm{He}\end{array}$

$\begin{array}{llllllll}\text { total mol \% } & 1.6 & 0 & 9.6 & 18.3 & 11.7 & 4.3 & 40.9\end{array}$

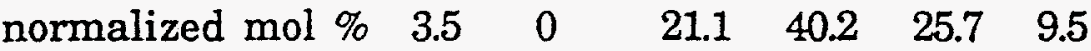

Residue Weight: $4.14 \mathrm{~g}$ (weight loss $=1.90 \mathrm{~g}$ )

Residue Composition: (by acid-base titration, Karl-Fisher titration and ${ }^{1} \mathrm{H}$ NMR)

$\left[\mathrm{H}^{+}\right]$

$\left[\mathrm{H}_{2} \mathrm{O}\right]: \quad *$

[TBP]: $\quad 9.74 \mathrm{mmol}(2.35 \mathrm{M})$

[DBP]: $\quad 3.06 \mathrm{mmol}(0.74 \mathrm{M})$

[MBP]: trace

$[\mathrm{BuONO} 2]: \quad 2.05 \mathrm{mmol}(0.50 \mathrm{M})$

[PrCOOH]: trace

[EtCOOH]: trace

$[\mathrm{MeCOOH}]: 0.57 \mathrm{mmol}(0.14 \mathrm{M})$

[HCOOH] trace

Condensate Composition:

Aqueous Phase $(0.74 \mathrm{~g})$

$\left[\mathrm{H}^{+}\right]$:

$6.93 \mathrm{mmol}(12.36 \mathrm{M})$

Organic Phase $(0.91 \mathrm{~g})$

$\left[\mathrm{BuONO}_{2}\right]: \quad 7.25 \mathrm{mmol}(8.20 \mathrm{M})$

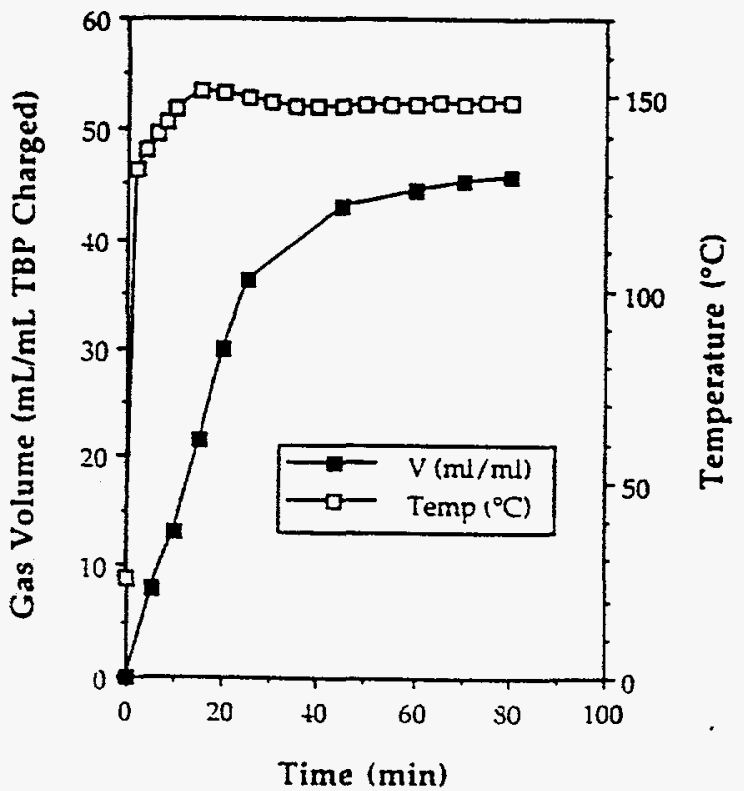

\section{Material Balance}

Not available due to the incompleteness of this set of data

* Not available because of solid residue 


\section{Results of Experiment No. 150 (Const. Pressure, Two Phase)}

\section{Reaction Conditions:}

Bath Temperature: $\quad 150^{\circ} \mathrm{C}$

Sample Volume: $\quad 2.5 \mathrm{~mL}$ each phase

Distillation/Refluxing $\mathrm{R}$
Acid coiıcentration: $\quad 6 \mathrm{M}$ Atmosphere: Fielium

\section{Before Reaction:}

Sample Weight: $2.65 \mathrm{~g}$ of organic and $3.51 \mathrm{~g}$ of aqueous phase

$\left[\mathrm{H}^{+}\right]$:

$\left[\mathrm{H}_{2} \mathrm{O}\right]$ :

[TBP]:
$14.5 \mathrm{mmol}$ (5.80 M, organic) and $36.13 \mathrm{mmol}$ (14.45 $\mathrm{M}$, aqueous)

$4.70 \mathrm{mmol}$ (1.88 M, organic)

$6.65 \mathrm{mmol}(2.66 \mathrm{M})$

\section{After Reaction:}

Total Gas Volume:

Gas Composition: total $\mathrm{mol} \%$ normalized $\mathrm{mol} \%$ $750 \mathrm{~mL}\left(25^{\circ} \mathrm{C}, 1 \mathrm{~atm}\right)$

$\begin{array}{llllllll}\mathrm{N}_{2} & \mathrm{O}_{2} & \mathrm{CO} & \mathrm{CO}_{2} & \mathrm{NO} & \mathrm{N}_{2} \mathrm{O} & \mathrm{He}\end{array}$ $\begin{array}{lllllll}6.9 & 0 & 0 & 24.2 & 40.1 & 6.4 & 20.7\end{array}$ Residue Weight: $1.91 \mathrm{~g}$ of organic (loss $=0.74 \mathrm{~g}$ ) and $3.05 \mathrm{~g}$ of aqueous (loss $=0.56 \mathrm{~g}$ ) Residue Composition: (by acid-base titration, Karl-Fisher titration and ${ }^{1} \mathrm{H}$ NMR) Organic Phase

$\left[\mathrm{H}^{+}\right]: \quad 10.51 \mathrm{mmol}(5.50 \mathrm{M})$

$\left[\mathrm{H}_{2} \mathrm{O}\right]: \quad 6.25 \mathrm{mmol}(3.27 \mathrm{M})$

[TBP]: $\quad 3.40 \mathrm{mmol}(1.78 \mathrm{M})$

[DBP]: $\quad 3.25 \mathrm{mmol}(1.70 \mathrm{M})$

[MBP]: trace

$\left[\mathrm{BuONO}_{2}\right]: \quad 0.17 \mathrm{mmol}(0.09 \mathrm{M})$ [PrCOOH]: trace [EtCOOH]: $0.35 \mathrm{mmol}(0.18 \mathrm{M})$ [MeCOOH]: $0.19 \mathrm{mmol}(0.10 \mathrm{M})$ [HCOOH]: trace Aqueous Phase

$\left[\mathrm{H}^{+}\right]$: $22.02 \mathrm{mmol}(9.24 \mathrm{M})$

$\left[\mathrm{BuONO}_{2}\right]$ : trace

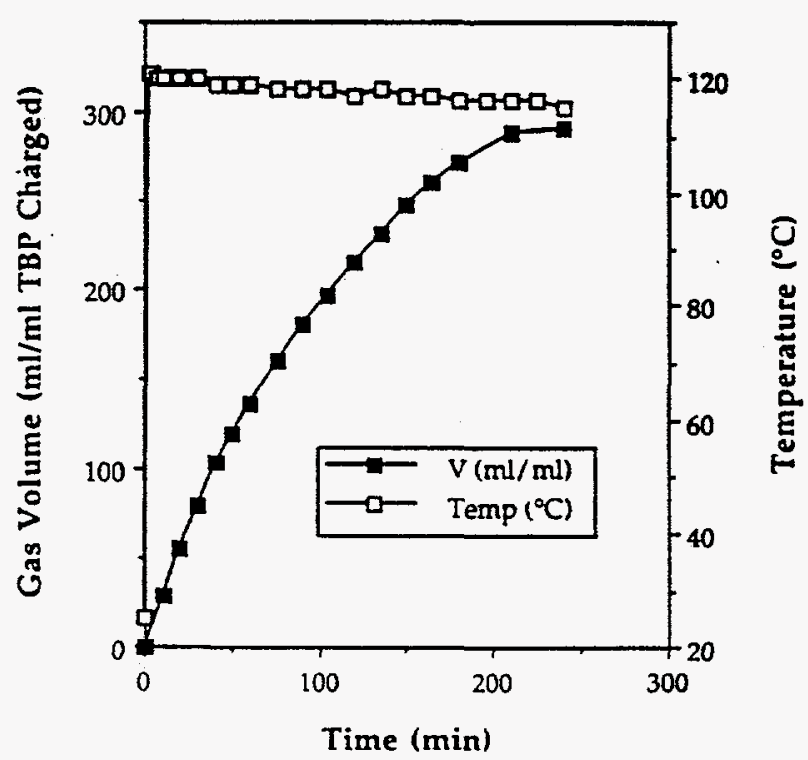

\section{Material Balance}

Carbon loss from residue $=10.89 \mathrm{mmol}$ Carbon present in gas $=9.58 \mathrm{mmol}$ Nitrogen loss from residue $=21.72 \mathrm{mmol}$ Nitrogen present in gas $=26.36 \mathrm{mmol}$ 
WSRC-RP-95-259

\section{Results of Experiment No. 151 (Const. Pressure, Two Phase)}

\section{Reaction Conditions:}

Bath Temperature: $\quad 150^{\circ} \mathrm{C}$

Sample Volume: $2.0 \mathrm{~mL}$ of each phase

Distillation/Refluxing $D$

\section{Before Reaction:}

Sample Weight: $2.04 \mathrm{~g}$ of organic and $2.46 \mathrm{~g}$ of aqueous phase
$\left[\mathrm{H}^{+}\right]$:
$6.00 \mathrm{mmol}$ (3.00 M, organic) and $11.50 \mathrm{mmol}$ (5.75 $\mathrm{M}$, aqueous)
[ $\left.\mathrm{H}_{2} \mathrm{O}\right]$ :
$2.96 \mathrm{mmol}$ (1.48 M, organic) [TBP]:
$5.68 \mathrm{mmol}(2.84 \mathrm{M})$

After Reaction:

Total Gas Volume: $\quad 101 \mathrm{~mL}\left(25^{\circ} \mathrm{C}, 1 \mathrm{~atm}\right)$

$\begin{array}{lllllllll}\text { Gas Composition: } & \mathrm{N}_{2} & \mathrm{O}_{2} & \mathrm{CO} & \mathrm{CO}_{2} & \mathrm{NO} & \mathrm{N}_{2} \mathrm{O} & \mathrm{He}\end{array}$

$\begin{array}{llllllll}\text { total } \mathrm{mol} \% & 4.5 & 0 & 8.8 & 16.5 & 20.8 & 3.6 & 37.7\end{array}$

normalized mol \% $8.3 \quad 0 \quad 16.2 \quad 30.4 \quad 38.4 \quad 6.6$

Residue Weight: $1.59 \mathrm{~g}$ of organic phase (loss $=0.45 \mathrm{~g}$ ) and no aqueous phase

Residue Compositions: (by acid-base titration, Karl-Fisher titration and ${ }^{1} \mathrm{H}$ NMR)

$\left[\mathrm{H}^{+}\right]$:

[TBP]:

$3.91 \mathrm{mmol}(2.46 \mathrm{M})$

[DBP]:

$3.13 \mathrm{mmol}(1.97 \mathrm{M})$

[MBP]:

$2.04 \mathrm{mmol}(1.28 \mathrm{M})$

[BuONO${ }_{2}$ ]: $\quad 0.37 \mathrm{mmol}(0.23 \mathrm{M})$

[Pr-COOH]: trace

[Et-COOH]: $\quad 0.24 \mathrm{mmol}(0.15 \mathrm{M})$

$\left[\mathrm{CH}_{3} \mathrm{COOH}\right]: \quad 0.09 \mathrm{mmol}(0.06 \mathrm{M})$

[HCOOH]: $\quad 0.08 \mathrm{mmol}(0.05 \mathrm{M})$

Condensate Compositions:

Aqueous Phase (2.37 g)

$\left[\mathrm{H}^{+}\right]$:

$11.5 \mathrm{mmol}(5.80 \mathrm{M})$

$[\mathrm{MeCOOH}]: \quad 0.29 \mathrm{mmol}(0.15 \mathrm{M})$

Organic Phase $(0.14 \mathrm{~g}$ )

[BuONO$\left.{ }_{2}\right]: \quad 1.12 \mathrm{mmol}(8.23 \mathrm{M})$

\section{Material Balance}

Carbon loss from residue $=5.30 \mathrm{mmol}$

Nitrogen loss from residue $=4.36 \mathrm{mmol}$
$\left[\mathrm{H}_{2} \mathrm{O}\right]: \quad 0.65 \mathrm{mmol}(0.41 \mathrm{M})$

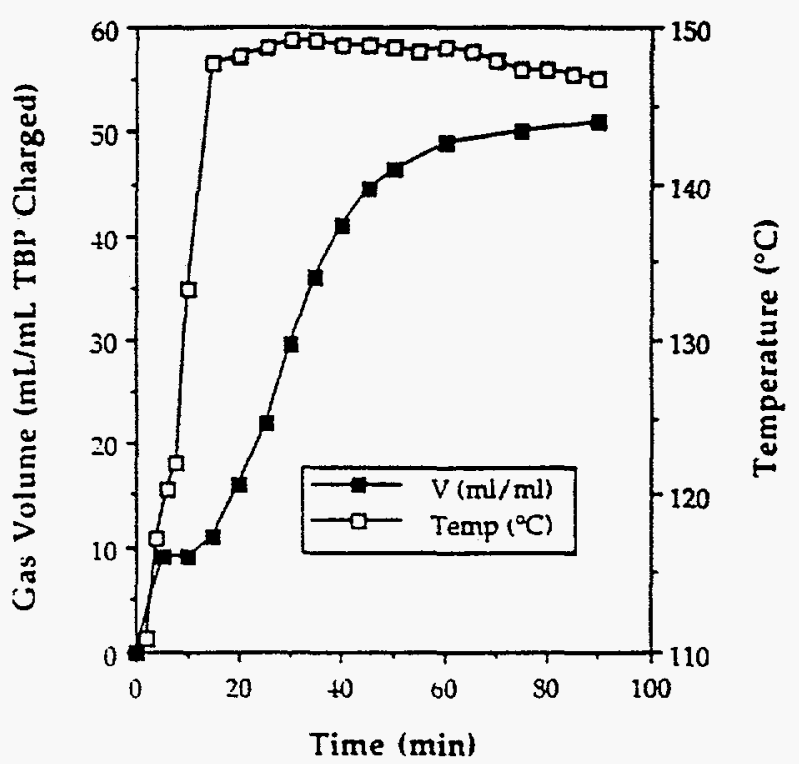

Carbon present in gas $=1.92 \mathrm{mmol}$ Nitrogen present in gas $=2.82 \mathrm{mmol}$ 
WSRC-RP-95-259

\section{Results of Experiment No. 152 (Const. Pressure, Two Phase)}

Reaction Conditions:

Bath Temperature: $\quad 150^{\circ} \mathrm{C}$

Acid concentration: $\quad 6 \mathrm{M}$

Sample Volume:

$2.0 \mathrm{~mL}$ of each phase

Atmosphere:

Helium

Distillation/Refluxing

$\mathrm{D}$

Before Reaction:

Sample Weight: $2.20 \mathrm{~g}$ of organic and $2.81 \mathrm{~g}$ of aqueous phase

$[\mathrm{H}+]$ :

$11.50 \mathrm{mmol}$ (5.75 $\mathrm{M}$, organic) and $30.08 \mathrm{mmol}$ (15.04 $\mathrm{M}$, aqueous)

$\left[\mathrm{H}_{2} \mathrm{O}\right]:$

$3.76 \mathrm{mmol}$ (1.88 M, organic)

[TBP]:

$5.32 \mathrm{mmol}(2.66 \mathrm{M})$

After Reaction:

Total Gas Volume: $\quad 85 \mathrm{~mL}\left(25^{\circ} \mathrm{C}, 1 \mathrm{~atm}\right)$

$\begin{array}{lllllllll}\text { Gas Composition: } & \mathrm{N}_{2} & \mathrm{O}_{2} & \mathrm{CO} & \mathrm{CO}_{2} & \mathrm{NO} & \mathrm{N}_{2} \mathrm{O} & \mathrm{He}\end{array}$ $\begin{array}{llllllll}\text { total } \mathrm{mol} \mathrm{\%} & 2.0 & 0 & 5.4 & 13.1 & 11.5 & 3.2 & 41.9\end{array}$

normalized mol \% $5.7 \quad 0 \quad 15.3 \quad 37.2 \quad 32.7 \quad 9.1$

Residue Weight: $1.37 \mathrm{~g}$ of organic phase (loss $=0.83 \mathrm{~g}$ ) and no aqueous phase Residue Composition: (by acid-base titration, Karl-Fisher titration and ${ }^{1} \mathrm{H}$ NMR) $\left[\mathrm{H}^{+}\right]$ $3.37 \mathrm{mmol}(2.46 \mathrm{M})$ $\left[\mathrm{H}_{2} \mathrm{O}\right]$ :

$1.12 \mathrm{mmol}(0.82 \mathrm{M})$

[TBP]: $\quad 3.00 \mathrm{mmol}(2.19 \mathrm{M})$

[DBP]: $\quad 1.79 \mathrm{mmol}(1.31 \mathrm{M})$

[MBP]: $\quad 0.53 \mathrm{mmol}(0.39 \mathrm{M})$

[BuONO 2$]: \quad 0.25 \mathrm{mmol}(0.18 \mathrm{M})$

[PrCOOH]: trace

[EtCOOH]: $\quad 0.24 \mathrm{mmol}(0.18 \mathrm{M})$

[MeCOOH]: $0.12 \mathrm{mmol}(0.09 \mathrm{M})$

[HCOOH]: $\quad 0.05 \mathrm{mmol}(0.04 \mathrm{M})$

Condensate Composition:

Aqueous Phase $(3.09 \mathrm{~g})$

$\left[\mathrm{H}^{+}\right]$:

$31.2 \mathrm{mmol}(14.1 \mathrm{M})$

[EtCOOH]: $0.94 \mathrm{mmol}(0.42 \mathrm{M})$

[MeCOOH]: $0.29 \mathrm{mmol}(0.13 \mathrm{M})$

Organic Phase $(0.04 \mathrm{~g})$

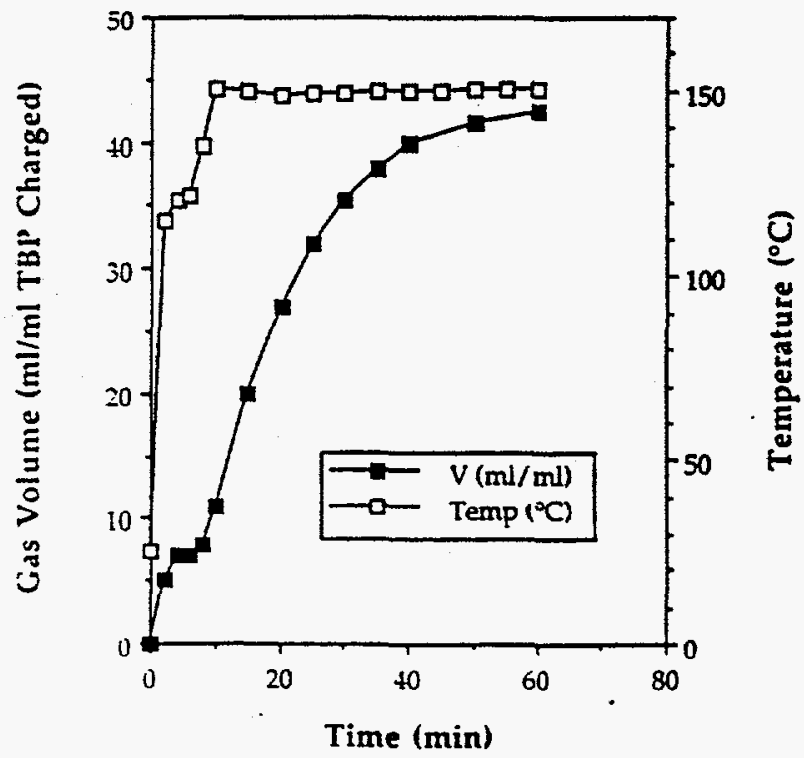

$\left[\mathrm{BuONO}_{2}\right]: \quad 0.32 \mathrm{mmol}(8.23 \mathrm{M})$

Material Balance

Carbon loss from residue $=4.71 \mathrm{mmol}$

Carbon present in gas $=1.21 \mathrm{mmol}$

Nitrogen loss from residue $=10.93 \mathrm{mmol}$

Nitrogen present in gas $=2.18 \mathrm{mmol}$ 
WSRC-RP-95-259

\section{Results of Experiment No. 153 (Const. Pressure, Two Phase)}

Bath Temperature: $\quad 180^{\circ} \mathrm{C} \quad$ Acid concentration: $\quad 6 \mathrm{M}$

Sample Volume: $\quad 2.0 \mathrm{~mL}$ of each phase Atmosphere: Helium

Distillation/Refluxing $\mathrm{D}$

\section{Before Reaction:}

Sample Weight: $2.20 \mathrm{~g}$ of organic and $2.83 \mathrm{~g}$ of aqueous phase

$\left[\mathrm{H}^{+}\right]$: $\quad 11.50 \mathrm{mmol}(5.75 \mathrm{M}$, organic) and $30.08 \mathrm{mmol}(15.04 \mathrm{M}$, aqueous)

$\left[\mathrm{H}_{2} \mathrm{O}\right]: \quad 3.76 \mathrm{mmol}(1.88 \mathrm{M}$, organic) $\quad$ [TBP]: $\quad 5.32 \mathrm{mmol}(2.66 \mathrm{M})$

\section{After Reaction:}

Sample Weight: $1.32 \mathrm{~g}$ of organic phase (loss $=0.88 \mathrm{~g}$ ) and no aqueous phase

Total Gas Volume: $\quad 75 \mathrm{~mL}\left(25^{\circ} \mathrm{C}, 1 \mathrm{~atm}\right)$

$\begin{array}{llllllll}\text { Gas Composition: } & \mathrm{N}_{2} & \mathrm{O}_{2} & \mathrm{CO} & \mathrm{CO}_{2} & \mathrm{NO} & \mathrm{N}_{2} \mathrm{O} & \mathrm{He}\end{array}$

$\begin{array}{llllllll}\text { total } \mathrm{mol} \mathrm{\%} & 1.3 & 0 & 2.3 & 9.6 & 15.2 & 1.8 & 46.9\end{array}$

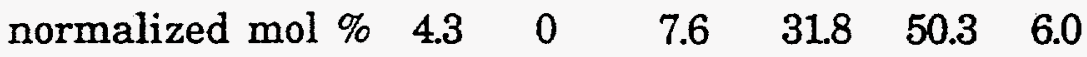

Residue Composition: (by acid-base titration, Karl-Fisher titration and ${ }^{1} \mathrm{H}$ NMR)

$\left[\mathrm{H}^{+}\right]$: $\quad 2.98 \mathrm{mmol}(2.26 \mathrm{M})$

$\left[\mathrm{H}_{2} \mathrm{O}\right]: \quad 0.90 \mathrm{mmol}(0.68 \mathrm{M})$

[TBP]: $\quad 3.02 \mathrm{mmol}(2.29 \mathrm{M})$

[DBP]: $\quad 1.76 \mathrm{mmol}(1.33 \mathrm{M})$

[MBP]: $\quad 0.54 \mathrm{mmol} \cdot(0.41 \mathrm{M})$

$\left[\mathrm{BuONO}_{2}\right]:$ trace

[EtCOOH]: $0.21 \mathrm{mmol}(0.16 \mathrm{M})$

[MeCOOH]: $0.10 \mathrm{mmol}(0.08 \mathrm{M})$

[HCOOH]: $\quad 0.07 \mathrm{mmol}(0.05 \mathrm{M})$

Condensate Composition:

Aqueous Phase (3.08 g)

$\left[\mathrm{H}^{+}\right]$:

$31.57 \mathrm{mmol}(14.35 \mathrm{M})$

[EtCOOH]: $0.92 \mathrm{mmol}(0.42 \mathrm{M})$

[MeCOOH]: $0.31 \mathrm{mmol}(0.14 \mathrm{M})$

Organic Phase $(0.04 \mathrm{~g})$

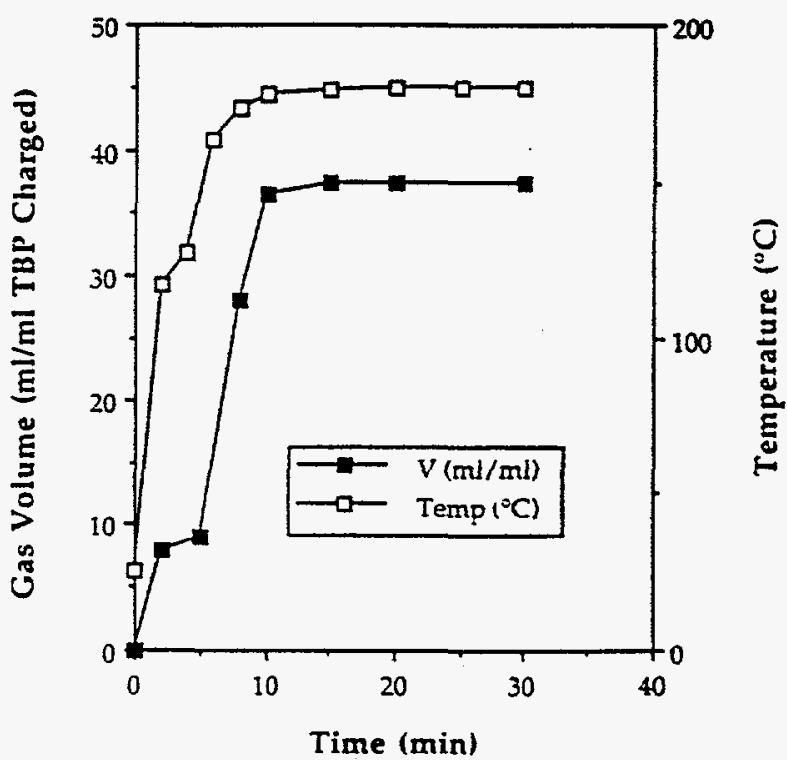

[BuONO${ }_{2}$ ]: $0.34 \mathrm{mmol}(8.74 \mathrm{M})$

\section{Material Balance}

Carbon loss in residue $=5.72 \mathrm{mmol}$

Carbon present in gas $=1.21 \mathrm{mmol}$

Nitrogen loss in residue $=10.9 \mathrm{mmol}$

Nitrogen present in gas $=2.18 \mathrm{mmol}$ 
WSRC-RP-95-259

\section{Results of Experiment No. 154 (Const. Pressure, Two Phase)}

\section{Reaction Conditions:}

Bath Temperature: $\quad 130^{\circ} \mathrm{C}$

Acid concentration: $\quad 6 \mathrm{M}$

Sample Volume:

$2.0 \mathrm{~mL}$ of each phase

Atmosphere:

Helium

Distillation/Refluxing

$\mathrm{D}$

\section{Before Reaction:}

Sample Weight: $2.19 \mathrm{~g}$ of organic and $2.82 \mathrm{~g}$ of aqueous phase

$\left[\mathrm{H}^{+}\right]$: $\quad 11.50 \mathrm{mmol}(5.75 \mathrm{M}$, organic) and $30.08 \mathrm{mmol}$ (15.04 M, aqueous)

$\left[\mathrm{H}_{2} \mathrm{O}\right]: \quad 3.76 \mathrm{mmol}(1.88 \mathrm{M}$, organic) $\quad$ [TBP]: $5.32 \mathrm{mmol}(2.66 \mathrm{M})$

\section{After Reaction:}

Total Gas Volume: $\quad 167 \mathrm{~mL}\left(25^{\circ} \mathrm{C}, 1 \mathrm{~atm}\right)$

$\begin{array}{lllllllll}\text { Gas Composition: } & \mathrm{N}_{2} & \mathrm{O}_{2} & \mathrm{CO} & \mathrm{CO}_{2} & \mathrm{NO} & \mathrm{N}_{2} \mathrm{O} & \mathrm{He}\end{array}$

$\begin{array}{llllllll}\text { total } \mathrm{mol} \mathrm{\%} & 4.8 & 0 & 1.9 & 4.0 & 0.8 & 0.8 & 69.9\end{array}$

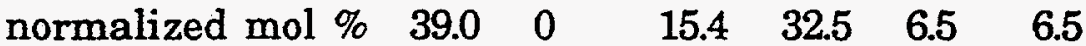

Residue Weight: $1.14 \mathrm{~g}$ of organic phase (loss $=1.05 \mathrm{~g}$ ) and no aqueous phase Residue Composition: (by acid-base titration, Karl-Fisher titration and ${ }^{1} \mathrm{H}$ NMR)

$[\mathrm{H}+]: \quad 4.71 \mathrm{mmol}(4.13 \mathrm{M})$

[TBP]: $\quad 2.16 \mathrm{mmol}(1.89 \mathrm{M})$

[DBP]: $\quad 2.50 \mathrm{mmol}(2.19 \mathrm{M})$

[MBP]: $\quad 0.66 \mathrm{mmol}(0.58 \mathrm{M})$

$\left[\mathrm{BuONO}_{2}\right]: \quad 0.16 \mathrm{mmol}(0.14 \mathrm{M})$

[PrCOOH]: trace

[EtCOOH]: $\quad 0.14 \mathrm{mmol}(0.12 \mathrm{M})$

[MeCOOH]: $0.09 \mathrm{mmol}(0.08 \mathrm{M})$

[HCOOH]: $\quad 0.02 \mathrm{mmol}(0.02 \mathrm{M})$

Condensate Composition:

Aqueous Phase (2.75 g)

$\left[\mathrm{H}^{+}\right]$:

$26.83 \mathrm{mmol}(12.88 \mathrm{M})$

[EtCOOH]: $\quad 1.13 \mathrm{mmol}(0.55 \mathrm{M})$

[MeCOOH]: $0.56 \mathrm{mmol}(0.27 \mathrm{M})$

Organic Phase $(0.18 \mathrm{~g})$

$\left[\mathrm{BuONO}_{2}\right] \quad 1.44 \mathrm{mmol}(8.63 \mathrm{M})$

\section{Material Balance}

Carbon loss from residue $=3.75 \mathrm{mmol}$

Nitrogen loss from residue $=14.20 \mathrm{mmol}$
$\left[\mathrm{H}_{2} \mathrm{O}\right]: 0.78 \mathrm{mmol}(0.68 \mathrm{M})$

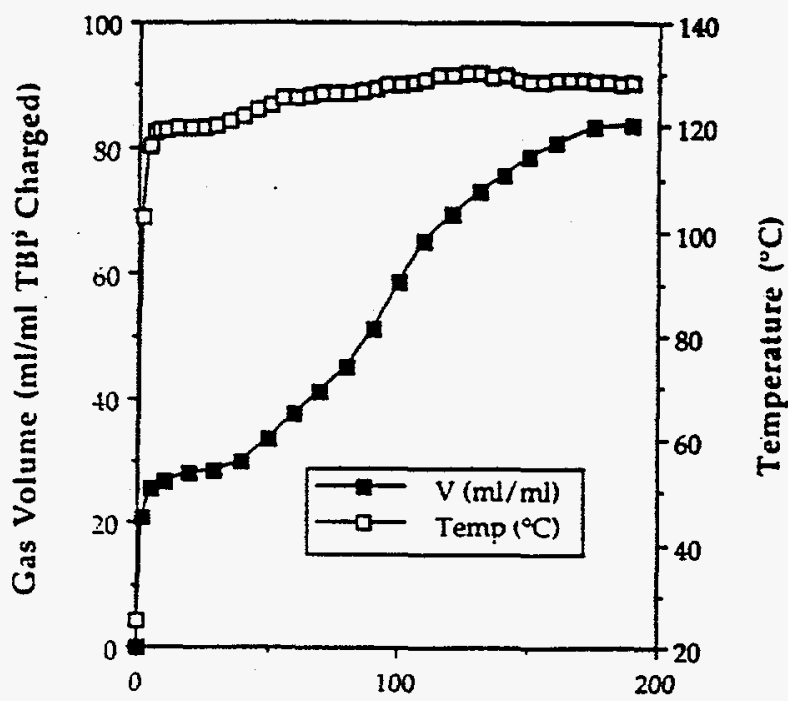

Time (min)

Carbon present in gas $=3.27 \mathrm{mmol}$ Nitrogen present in gas $=6.66 \mathrm{mmol}$ 
WSRC-RP-95-259

\section{Results of Experiment No. 155 (Const. Volume, Two Phase)}

Reaction Conditions:

Bath Temperature:

Sample Volume:

Before Reaction:

\author{
$150^{\circ} \mathrm{C}$
}

$2 \mathrm{ml}$ of each phase
Acid concentration:

Atmosphere:
$6 \mathrm{M}$

Helium

Sample Weight: $2.18 \mathrm{~g}$ of organic and $2.80 \mathrm{~g}$ of aqueous phase

$[\mathrm{H}+]$ : $\quad 11.50 \mathrm{mmol}(5.75 \mathrm{M}$, organic) and $30.08 \mathrm{mmol}(15.04 \mathrm{M}$, aqueous)

[ $\left.\mathrm{H}_{2} \mathrm{O}\right]: \quad 3.76 \mathrm{mmol}(1.88 \mathrm{M}$, organic) [TBP]: $5.32 \mathrm{mmol}(2.66 \mathrm{M})$

\section{After Reaction:}

Total Gas Pressure: $\quad 17.5$ atms $\left(25^{\circ} \mathrm{C}\right.$, volume $\left.=58 \mathrm{ml}\right)$

$\begin{array}{lllllllll}\text { Gas Composition: } & \mathrm{N}_{2} & \mathrm{O}_{2} & \mathrm{CO} & \mathrm{CO}_{2} & \mathrm{NO} & \mathrm{N}_{2} \mathrm{O} & \mathrm{He}\end{array}$

$\begin{array}{llllllll}\text { total } \mathrm{mol} \% & 5.3 & 0 & 14.3 & 36.4 & 23.6 & 12.4 & 4.6\end{array}$

$\begin{array}{lllllll}\text { normalized } \mathrm{mol} \% & 5.8 & 0 & 15.5 & 39.6 & 25.7 & 13.5\end{array}$

Residue Weight: $1.09 \mathrm{~g}$ of organic phase (loss $=1.09 \mathrm{~g}$ ) and $2.03 \mathrm{~g}$ of aqueous phase (loss $=0.77 \mathrm{~g}$ )

Residue Composition: (by acid-base titration, Karl-Fisher titration and ${ }^{1} \mathrm{H}$ NMR) Organic Phase (1.09 g)

$\left[\mathrm{H}^{+}\right]: \quad 5.79 \mathrm{mmol}\left(5.31 \mathrm{M}\right.$, organic) $\quad\left[\mathrm{H}_{2} \mathrm{O}\right]: 3.96 \mathrm{mmol}$ (3.63 M, organic)

[TBP]: $\quad 1.05 \mathrm{mmol}(0.96 \mathrm{M})$

[DBP]: $\quad 2.10 \mathrm{mmol}(1.93 \mathrm{M})$

[MBP]: $\quad 1.19 \mathrm{mmol}(1.09 \mathrm{M})$

[BuONO 2$]: 0.32 \mathrm{mmol}(0.29 \mathrm{M})$

[PrCOOH]: $1.84 \mathrm{mmol}(1.69 \mathrm{M})$

[EtCOOH]: $1.84 \mathrm{mmol}(1.69 \mathrm{M})$

[MeCOOH]: $1.14 \mathrm{mmol}(1.05 \mathrm{M})$

[HCOOH]: trace

Aqueous Phase (2.03 g)

$\left[\mathrm{H}^{+}\right]$:

$9.31 \mathrm{mmol}(5.41 \mathrm{M})$

[EtCOOH]: $\quad 1.62 \mathrm{mmol}(1.12 \mathrm{M})$

[MBP]: $\quad 0.97 \mathrm{mmol}(0.69 \mathrm{M})$

[MeCOOH]: $1.63 \mathrm{mmol}(1.12 \mathrm{M}$ )

\section{Material Balance}

Carbon loss from residue $=1.12 \mathrm{mmol}$

Nitrogen loss in residue $=37.15 \mathrm{mmol}$

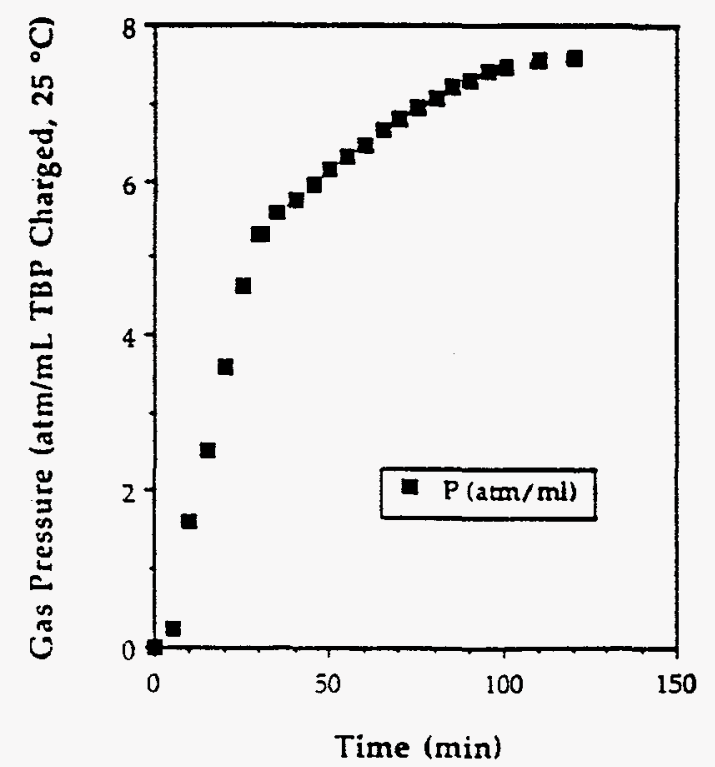

Carbon present in gas $=22.89 \mathrm{mmol}$ Nitrogen present in gas $=26.71 \mathrm{mmol}$ 
WSRC-RP-95-259

\section{Results of Experiment No. 156 (Const. Volume, Two Phase)}

Bath Temperature:

$150^{\circ} \mathrm{C}$

Acid concentration:

$3 \mathrm{M}$

Sample Volume:

$2 \mathrm{ml}$ of each phase

Atmosphere:

Helium

\section{Before Reaction:}

Sample Weight: $2.08 \mathrm{~g}$ of organic and $2.44 \mathrm{~g}$ of aqueous phase

$\left[\mathrm{H}^{+}\right]$

$6.00 \mathrm{mmol}(3.00 \mathrm{M}$, organic) and $11.50 \mathrm{mmol}(5.75 \mathrm{M}$, aqueous)

$\left[\mathrm{H}_{2} \mathrm{O}\right]:$

$2.96 \mathrm{mmol}$ (1.48 M, organic)

[TBP]:

$5.68 \mathrm{mmol}(2.84 \mathrm{M})$

After Reaction:

Total Gas Pressure: $\quad 6.0$ atms $\left(25^{\circ} \mathrm{C}, 58 \mathrm{~mL}\right.$ volume $)$

$\begin{array}{lllllllll}\text { Gas Composition: } & \mathrm{N}_{2} & \mathrm{O}_{2} & \mathrm{CO} & \mathrm{CO}_{2} & \mathrm{NO} & \mathrm{N}_{2} \mathrm{O} & \mathrm{He}\end{array}$

$\begin{array}{llllllll}\text { total mol \% } & 5.5 & 0 & 17.0 & 36.3 & 14.1 & 12.5 & 13.9 \\ \text { normalized mol \% } & 6.4 & 0 & 19.9 & 42.5 & 16.5 & 14.6 & \end{array}$

Residue Weight: $1.85 \mathrm{~g}$ of organic phase (loss $=0.23 \mathrm{~g}$ ) and $2.06 \mathrm{~g}$ of aqueous phase (loss $=0.38 \mathrm{~g})$

Residue Composition: (by acid-base titration, Karl-Fisher titration and ${ }^{1} \mathrm{H}$ NMR) Organic Phase

$\left[\mathrm{H}^{+}\right]: \quad 6.18 \mathrm{mmol}(3.34 \mathrm{M})$

$\left[\mathrm{H}_{2} \mathrm{O}\right]: \quad 5.03 \mathrm{mmol}(2.72 \mathrm{M})$

[TBP]: $\quad 2.58 \mathrm{mmol}(1.39 \mathrm{M})$

[DBP]: $\quad 1.69 \mathrm{mmol}(0.91 \mathrm{M})$

[MBP]: $\quad 1.03 \mathrm{mmol}(0.56 \mathrm{M})$

$\left[\mathrm{BuONO}_{2}\right]: \quad 1.31 \mathrm{mmol}(0.71 \mathrm{M})$

[PrCOOH]: trace

[EtCOOH]: $\quad 1.27 \mathrm{mmol}(0.69 \mathrm{M})$

[MeCOOH]: $0.31 \mathrm{mmol}(0.17 \mathrm{M})$

[HCOOH]: trace

Aqueous Phase

$\left[\mathrm{H}^{+}\right]$:

$4.90 \mathrm{mmol}(2.56 \mathrm{M})$

[EtCOOH]: $\quad 0.49 \mathrm{mmol}(0.26 \mathrm{M})$

[MBP]: $\quad 0.39 \mathrm{mmol}(0.20 \mathrm{M})$

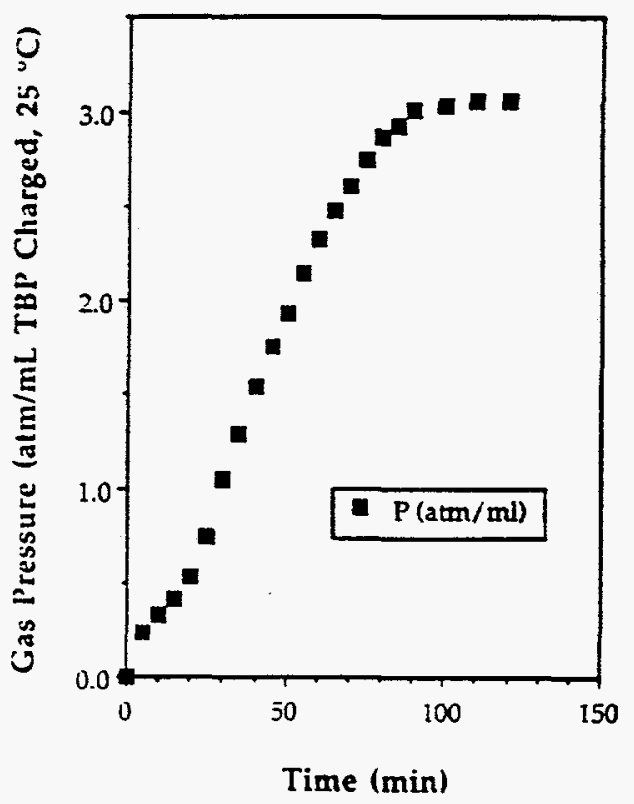

[MeCOOH]: $0.61 \mathrm{mmol}(0.32 \mathrm{M})$

\section{Material Balance}

Carbon loss from residue $=5.76 \mathrm{mmol}$

Carbon present in gas $=8.89 \mathrm{mmol}$

Nitrogen loss from residue $=11.54 \mathrm{mmol}$

Nitrogen present in gas $=8.33 \mathrm{mmol}$ 
WSRC-RP-95-259

\section{Results of Experiment No. 157 (Const. Volume, Two Phase)}

Reaction Conditions:

Sath Temperature: $\quad 130^{\circ} \mathrm{C}$

Sample Volume:

$2 \mathrm{ml}$ of each phase

Acid concentration:

$6 \mathrm{M}$

Before Reaction:

Sample Weight: $2.16 \mathrm{~g}$ of organic and $2.85 \mathrm{~g}$ of aqueous phase

$\left[\mathrm{H}^{+}\right]$: $\quad 11.50 \mathrm{mmol}(5.75 \mathrm{M}$, organic) and $30.08 \mathrm{mmol}$ (15.04 M, aqueous)

$\left[\mathrm{H}_{2} \mathrm{O}\right]: \quad 3.76 \mathrm{mmol}(1.88 \mathrm{M}$, organic) $\quad$ [TBP]: $\quad 5.32 \mathrm{mmol}(2.66 \mathrm{M})$

After Reaction:

Total Gas Pressure: $\quad 12.1$ atms $\left(25^{\circ} \mathrm{C}, 58 \mathrm{~mL}\right.$ volume)

$\begin{array}{llllllll}\text { Gas Composition: } & \mathrm{N}_{2} & \mathrm{O}_{2} & \mathrm{CO} & \mathrm{CO}_{2} & \mathrm{NO} & \mathrm{N}_{2} \mathrm{O} & \mathrm{He}\end{array}$ $\begin{array}{llllllll}\text { total mol \% } & 6.5 & 0 & 14.4 & 36.5 & 25.5 & 5.9 & 7.0\end{array}$ normalized mol \% $\quad 7.3 \quad 0 \quad 16.2 \quad 41.4 \quad 28.7 \quad 6.6$

Residue Weight: $1.58 \mathrm{~g}$ of organic phase (loss $=0.58 \mathrm{~g}$ ) and $2.10 \mathrm{~g}$ of aqueous phase (loss $=0.75 \mathrm{~g})$

Residue Composition: (by acid-base titration, Karl-Fisher titration and $1 \mathrm{H}$ NMR) Organic Phase ( $1.58 \mathrm{~g})$

$\left[\mathrm{H}^{+}\right]$: $\quad 9.01 \mathrm{mmol}(5.70 \mathrm{M})$

$\left[\mathrm{H}_{2} \mathrm{O}\right]: \quad 8.17 \mathrm{mmol}(5.17 \mathrm{M})$

[TBP]: $\quad 1.95 \mathrm{mmol}(1.23 \mathrm{M})$

[DBP]: $\quad 1.94 \mathrm{mmol}(1.23 \mathrm{M})$

[MBP]: $\quad 1.42 \mathrm{mmol}(0.90 \mathrm{M})$

$[\mathrm{BuONO} 2]: \quad 0.21 \mathrm{mmol}(0.13 \mathrm{M})$

[PrCOOH]: trace

[EtCOOH]: $1.30 \mathrm{mmol}(0.82 \mathrm{M})$

[MeCOOH]: $0.86 \mathrm{mmol}(0.54 \mathrm{M})$

[HCOOH]: trace

Aqueous Phase $(2.10 \mathrm{~g})$

$\left[\mathrm{H}^{+}\right]$:

$12.57 \mathrm{mmol}(7.18 \mathrm{M})$

[PrCOOH]: trace

[EtCOOH]: $0.65 \mathrm{mmol}(0.37 \mathrm{M})$

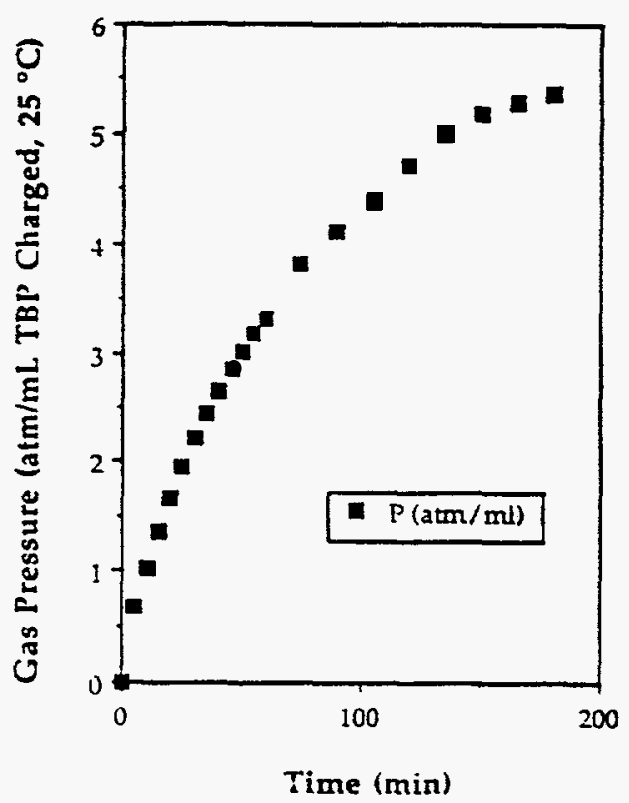

[MeCOOH]: $1.58 \mathrm{mmol}(0.90 \mathrm{M})$

Material Balance

Carbon loss from residue $=7.55 \mathrm{mmol}$

Carbon present in gas $=16.54 \mathrm{mmol}$

Nitrogen loss from residue $=28.96 \mathrm{mmol}$

Nitrogen present in gas $=16.23 \mathrm{mmol}$ 
Results of Experiment No. 158 (Const. Volume, Two Phase)

Reaction Conditions:

Bath Temperature: $\quad 180^{\circ} \mathrm{C}$

Acid concentration: $\quad 6 \mathrm{M}$

Sample Volume:

$2 \mathrm{ml}$ of each phase

Atmosphere:

Helium

Before Reaction:

Sample Weight: $2.18 \mathrm{~g}$ of organic and $2.82 \mathrm{~g}$ of aqueous phase

$\left[\mathrm{H}^{+}\right]$: $\quad 11.50 \mathrm{mmol}(5.75 \mathrm{M}$, organic) and $30.08 \mathrm{mmol}$ (15.04 M, aqueous)

$\left[\mathrm{H}_{2} \mathrm{O}\right]: \quad 3.76 \mathrm{mmol}(1.88 \mathrm{M}$, organic) $\quad$ [TBP]: $5.32 \mathrm{mmol}(2.66 \mathrm{M})$

After Reaction:

Total Gas Pressure: $\quad 17.1$ atms $\left(25^{\circ} \mathrm{C}\right.$, volume $\left.=58 \mathrm{ml}\right)$

Gas Composition: $\quad \mathrm{N}_{2} \quad \mathrm{O}_{2} \quad \mathrm{CO} \quad \mathrm{CO}_{2} \quad \mathrm{NO} \quad \mathrm{N}_{2} \mathrm{O} \quad \mathrm{He}$

$\begin{array}{llllllll}\text { total mol \% } & 8.6 & 0 & 21.9 & 41.4 & 3.4 & 17.3 & 5.4\end{array}$

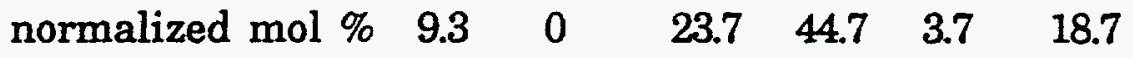

Residue Weight: $\quad 0.87 \mathrm{~g}$ of organic phase (loss $=1.31 \mathrm{~g}$ ) and $2.14 \mathrm{~g}$ of aqueous

$$
\text { phase (loss }=0.68 \mathrm{~g} \text { ) }
$$

Residue Composition: (by acid-base titration, Karl-Fisher titration and ${ }^{1} \mathrm{H}$ NMR) Organic Phase $(0.87 \mathrm{~g}$ )

$\left[\mathrm{H}^{+}\right]: \quad \quad 4.79 \mathrm{mmol}(5.50 \mathrm{M})$

[TBP]:

[DBP]:

[MBP]:

$\left[\mathrm{BuONO}_{2}\right]:$ *

[PrCOOH]: *

[EtCOOH]: *

[MeCOOH]: *

[HCOOH]: *

Aqueous Phase (2.14 g)

$\left[\mathrm{H}^{+}\right]: \quad \quad 10.16 \mathrm{mmol}(5.70 \mathrm{M})$

[PrCOOH]: $\quad 0.45 \mathrm{mmol}(0.25 \mathrm{M})$

[EtCOOH]: $1.03 \mathrm{mmol}(0.58 \mathrm{M})$

[MeCOOH]: $1.92 \mathrm{mmol}(1.08 \mathrm{M})$

[MBP]: $\quad 0.93 \mathrm{mmol}(0.52 \mathrm{M})$

$\left[\mathrm{H}_{2} \mathrm{O}\right]: \quad 3.68 \mathrm{mmol}(4.23 \mathrm{M})$

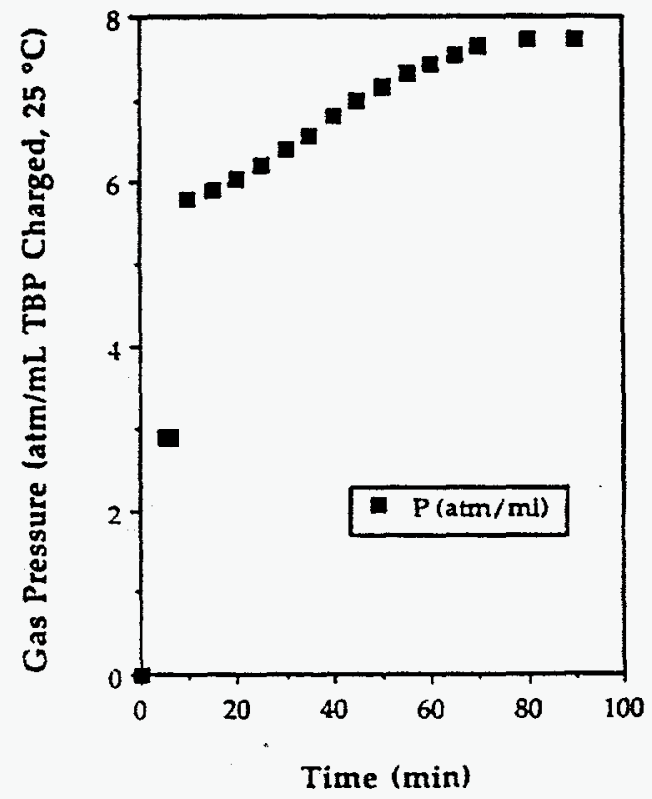

* The presence of paramagnetic material made ${ }^{1} \mathrm{H}$ NMR analysis unavailable 
WSRC-RP-95-259

\section{Results of Experiment No. 159 (Const. Pressure, Two Phase)}

\section{Reaction Conditions:}

Bath Temperature: $\quad 180^{\circ} \mathrm{C}$

Sample Volume:

$1.5 \mathrm{~mL}$ of each phase

Acid concentration:

$6 \mathrm{M}$

Distillation/Refluxing $R$

Atmosphere: Helium

Before Reaction:

Sample Weight: $1.61 \mathrm{~g}$ of organic and $2.13 \mathrm{~g}$ of aqueous phase

$\left[\mathrm{H}^{+}\right]$: $\quad 8.63 \mathrm{mmol}(5.75 \mathrm{M}$, organic) and $22.56 \mathrm{mmol}$ (15.04 M, aqueous)

$\left[\mathrm{H}_{2} \mathrm{O}\right]: \quad 2.82 \mathrm{mmol}(1.88 \mathrm{M}$, organic) $\quad[\mathrm{TBP}]: \quad 3.99 \mathrm{mmol}(2.66 \mathrm{M})$

\section{After Reaction:}

Total Gas Volume: $\quad 417 \mathrm{~mL}\left(25^{\circ} \mathrm{C}, 1 \mathrm{~atm}\right)$

$\begin{array}{llllllll}\text { Gas Composition: } & \mathrm{N}_{2} & \mathrm{O}_{2} & \mathrm{CO} & \mathrm{CO}_{2} & \mathrm{NO} & \mathrm{N}_{2} \mathrm{O} & \mathrm{He}\end{array}$ $\begin{array}{llllllll}\text { total mol \% } & 14.9 & 0 & 13.7 & 17.8 & 17.1 & 4.4 & 18.7\end{array}$

normalized mol \% $21.9 \quad 0 \quad 20.2 \quad 26.2 \quad 25.2 \quad 6.5$

Residue Weight: $1.09 \mathrm{~g}$ of organic phase (loss $=0.52 \mathrm{~g}$ ) and $1.72 \mathrm{~g}$ of aqueous

$$
\text { phase (loss }=0.31 \mathrm{~g})
$$

Residue Composition: (by acid-base titration, Karl-Fisher titration and ${ }^{1} \mathrm{H}$ NMR)

Organic Phase (1.09 g)

$\left[\mathrm{H}^{+}\right]$: $\quad 6.32 \mathrm{mmol}(5.80 \mathrm{M})$

$\left[\mathrm{H}_{2} \mathrm{O}\right]: \quad 3.56 \mathrm{mmol}(3.27 \mathrm{M})$

[TBP]: $\quad 1.90 \mathrm{mmol}(1.74 \mathrm{M})$

[DBP]: $\quad 1.82 \mathrm{mmol}(1.67 \mathrm{M})$

[MBP]: $\quad 0.27 \mathrm{mmol}(0.25 \mathrm{M})$

[BuONO 2$]: 0.29 \mathrm{mmol}(0.27 \mathrm{M})$

[PrCOOH]: trace

[EtCOOH]: $\quad 0.50 \mathrm{mmol}(0.46 \mathrm{M})$

[MeCOOH]: $0.29 \mathrm{mmol}(0.27 \mathrm{M})$

[HCOOH]: trace

Aqueous Phase (1.72 g)

$\left[\mathrm{H}^{+}\right]$:

$12.62 \mathrm{mmol}(9.54 \mathrm{M})$

[EtCOOH]: $\quad 0.94 \mathrm{mmol}(0.71 \mathrm{M})$

[MeCOOH]: $1.27 \mathrm{mmol}(0.96 \mathrm{M})$

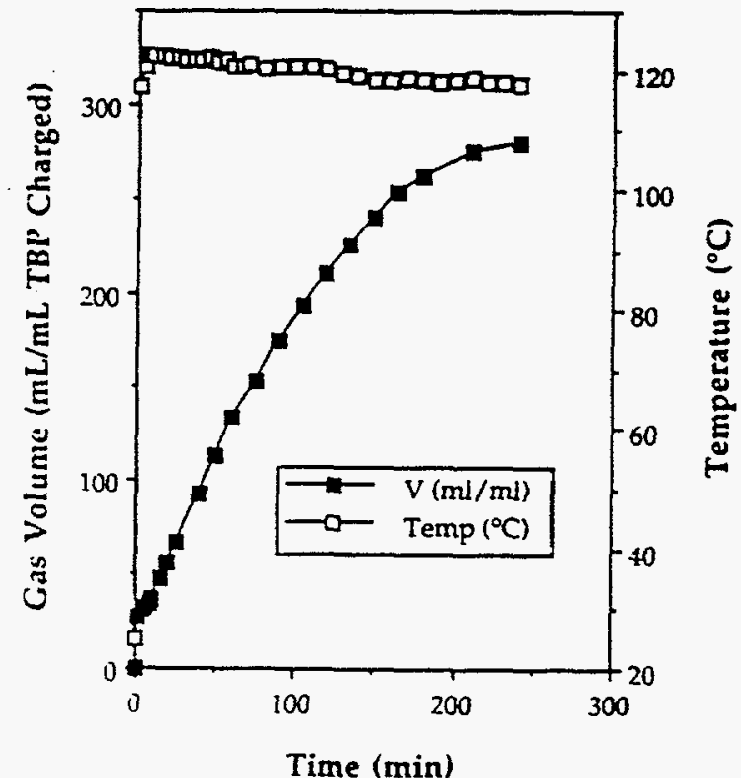

Carbon present in gas $=7.92 \mathrm{mmol}$ Nitrogen present in gas $=13.99 \mathrm{mmol}$ 
Results of Experiment No. 160 (Const. Pressure, Two Phase)

Reaction Conditions:

Bath Temperature: $\quad 130^{\circ} \mathrm{C}$

Sample Volume: $\quad 1.5 \mathrm{~mL}$ of eäh phase.

Acid concentration:

$6 \mathrm{M}$

Distillation/Refluxing $\mathrm{R}$

Atmosphere: Helium

Before Reaction:

Sample Weight: $1.63 \mathrm{~g}$ of organic and $2.15 \mathrm{~g}$ of aqueous phase

$\left[\mathrm{H}^{+}\right]$: $\quad 8.63 \mathrm{mmol}(5.75 \mathrm{M}$, organic) and $22.56 \mathrm{mmol}$ (15.04 M, aqueous)

[ $\left.\mathrm{H}_{2} \mathrm{O}\right]: \quad 2.82 \mathrm{mmol}(1.88 \mathrm{M}$, organic) $\quad$ [TBP]: $\quad 3.99 \mathrm{mmol}(2.66 \mathrm{M})$

After Reaction:

Total Gas Volume: $\quad 342 \mathrm{~mL}\left(25^{\circ} \mathrm{C}, 1 \mathrm{~atm}\right)$

$\begin{array}{lllllllll}\text { Gas Composition: } & \mathrm{N}_{2} & \mathrm{O}_{2} & \mathrm{CO} & \mathrm{CO}_{2} & \mathrm{NO} & \mathrm{N}_{2} \mathrm{O} & \mathrm{He}\end{array}$

$\begin{array}{llllllll}\text { total mol \% } & 16.5 & 0 & 16.4 & 16.2 & 10.5 & 4.4 & 24.3\end{array}$

normalized mol \% $25.8 \quad 0 \quad 25.6 \quad 25.3 \quad 16.4 \quad 6.9$

Residue Weight: $1.10 \mathrm{~g}$ of organic phase (loss $=0.53 \mathrm{~g}$ ) and $1.69 \mathrm{~g}$ of aqueous phase (loss $=0.46 \mathrm{~g}$ )

Residue Composition: (by acid-base titration, Karl-Fisher titration and ${ }^{1} \mathrm{H}$ NMR) Organic Phase (1.10 g)

$\left[\mathrm{H}^{+}\right]$: $\quad 6.05 \mathrm{mmol}(5.50 \mathrm{M})$

$\left[\mathrm{H}_{2} \mathrm{O}\right]: \quad 3.89 \mathrm{mmol}(3.54 \mathrm{M})$

[TBP]: $\quad 1.77 \mathrm{mmol}(1.61 \mathrm{M})$

[DBP]: $\quad 1.81 \mathrm{mmol}(1.64 \mathrm{M})$

[MBP]: $\quad 0.41 \mathrm{mmol}(0.38 \mathrm{M})$

[BuONO 2$]: 0.15 \mathrm{mmol}(0.14 \mathrm{M})$

[PrCOOH]: trace

[EtCOOH]: $\quad 0.41 \mathrm{mmol}(0.38 \mathrm{M})$

[MeCOOH]: $0.25 \mathrm{mmol}(0.22 \mathrm{M})$

[HCOOH]: trace

Aqueous Phase (1.69 g)

$\left[\mathrm{H}^{+}\right]: \quad 12.91 \mathrm{mmol}(9.93 \mathrm{M})$

[EtCOOH]: $0.70 \mathrm{mmol}(0.54 \mathrm{M})$

[MeCOOH]: $1.09 \mathrm{mmol}(0.84 \mathrm{M})$

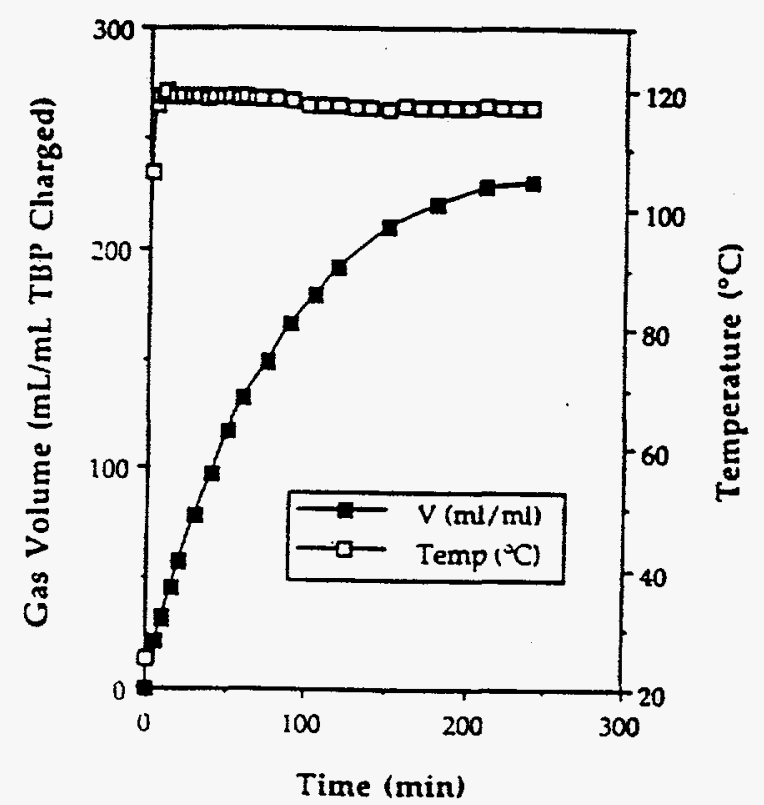

Material Balance

Carbon loss from residue $=3.91 \mathrm{mmol}$

Carbon present in gas $=7.13 \mathrm{mmol}$

Nitrogen loss from residue $=17.16 \mathrm{mmol}$

Nitrogen present in gas $=11.45 \mathrm{mmol}$ 


\section{Results of Experiment No. 161 (Const. Pressure, Two Phase)}

Reaction Conditions:

Bath Temperature: $\quad 150^{\circ} \mathrm{C}$

Sample Volume:

Catalyst:
$2.0 \mathrm{~mL}$ of each phase $\mathrm{Zr}^{4+}$
Acid concentration:

$6 \mathrm{M}$ Átrnosphere: Helium Distillation/Refluxing $\quad \mathrm{R}$

\section{Before Reaction:}

Sample Weight: $2.23 \mathrm{~g}$ of organic and $2.81 \mathrm{~g}$ of aqueous phase

$\left[\mathrm{H}^{+}\right]$: $\quad 11.60 \mathrm{mmol}(5.80 \mathrm{M}$, organic) and $30.90 \mathrm{mmol}$ (15.45 M, aqueous)

$\left[\mathrm{H}_{2} \mathrm{O}\right]: \quad 6.54 \mathrm{mmol}(3.27 \mathrm{M}$, organic) $\quad$ [TBP]: $\quad 5.12 \mathrm{mmol}(2.56 \mathrm{M})$

\section{After Reaction:}

Total Gas Volume: $\quad 82 \mathrm{~mL}\left(25^{\circ} \mathrm{C}, 1 \mathrm{~atm}\right)$

Gas Composition:

$\begin{array}{llllllll}\mathrm{N}_{2} & \mathrm{O}_{2} & \mathrm{CO} & \mathrm{CO}_{2} & \mathrm{NO} & \mathrm{N}_{2} \mathrm{O} & \mathrm{He}\end{array}$

total mol \%

$3.8 \quad 0$

8.4

$\begin{array}{llll}18.0 & 11.5 & 4.1 & 37.1\end{array}$

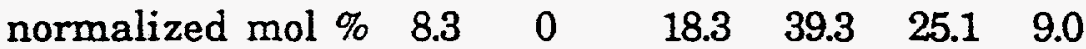

Residue Weight: $1.10 \mathrm{~g}$ of solid residue

Residue Composition: (by acid-base titration, Karl-Fisher titration and ${ }^{1} \mathrm{H}$ NMR) $[\mathrm{H}+]: \quad \quad \mathrm{N} / \mathrm{A}^{* *}$

$\left[\mathrm{H}_{2} \mathrm{O}\right]: \quad \mathrm{N} / \mathrm{A}^{* *}$

[TBP]: $\quad 2.93 \mathrm{mmol}$

[DBP]: $\quad 2.19 \mathrm{mmol}$

$[\mathrm{BuONO} 2]: \quad 0.86 \mathrm{mmol}$

[EtCOOH]: $0.35 \mathrm{mmol}$

[MeCOOH]: $0.16 \mathrm{mmol}$

[HCOOH]: $\quad 0.03 \mathrm{mmol}$

Condensate Composition:

Aqueous Phase $(3.09 \mathrm{~g})$

$\left[\mathrm{H}^{+}\right]$:

$31.03 \mathrm{mmol}(14.06 \mathrm{M})$

[MBP]: $\quad 0.34 \mathrm{mmol}(0.15 \mathrm{M})$

[EtCOOH]: $\quad 0.99 \mathrm{mmol}(0.45 \mathrm{M})$

[MeCOOH]: $0.23 \mathrm{mmol}(0.10 \mathrm{M})$

Organic Phase $(0.07 \mathrm{~g})$

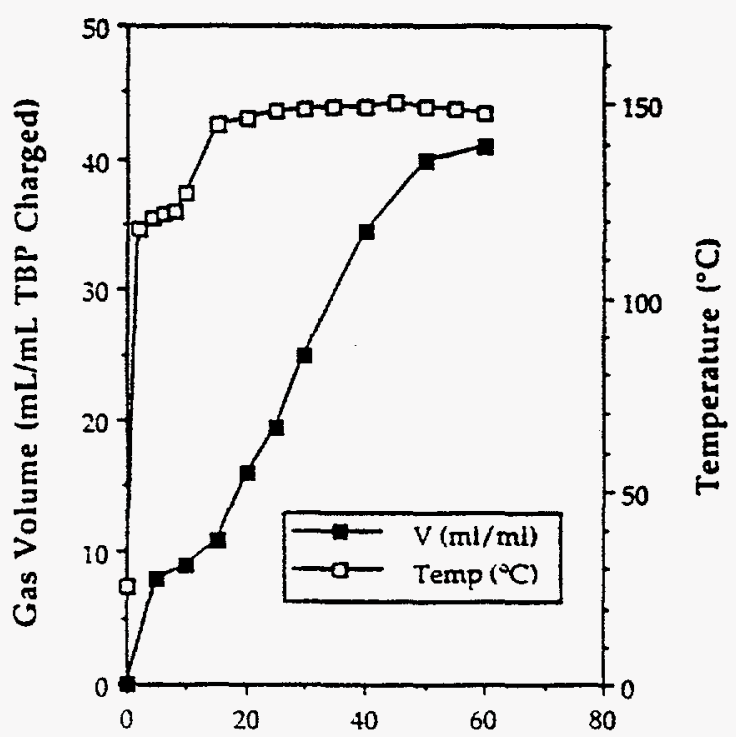

Time (min)

Carbon present in gas $=1.94 \mathrm{mmol}$

Carbon loss from residue $=0.85 \mathrm{mmol}$

** Not available because of solid residue 
WSRC-RP-95-259

\section{Results of Experiment No. 162 (Const. Volume, Two Phase)}

\section{Reaction Conditions:}

Bath Temperature:

Sample Volume:

Catalyst: $150^{\circ} \mathrm{C}$

$1 \mathrm{ml}$ of each phase

$\mathrm{Zr}^{4+}$
Acid concentration:

Atmosph?re:
$6 \mathrm{M}$

Pelium

\section{Before Reaction:}

Sample Weight: $1.12 \mathrm{~g}$ of organic and $1.44 \mathrm{~g}$ of aqueous phase

$\left[\mathrm{H}^{+}\right]$:

$5.80 \mathrm{mmol}(5.80 \mathrm{M}$, organic) and $14.45 \mathrm{mmol}$ (14.45 $\mathrm{M}$, aqueous)

$\left[\mathrm{H}_{2} \mathrm{O}\right]$ :

$3.27 \mathrm{mmol}$ (3.27 M, organic)

[TBP]:

\section{$2.56 \mathrm{mmol}(2.56 \mathrm{M})$}

\section{After Reaction:}

Total Gas Pressure: $\quad 7.9$ atms $\left(25^{\circ} \mathrm{C}, 58 \mathrm{~mL}\right.$ volume $)$.

$\begin{array}{llllllll}\text { Gas Composition: } & \mathrm{N}_{2} & \mathrm{O}_{2} & \mathrm{CO} & \mathrm{CO}_{2} & \mathrm{NO} & \mathrm{N}_{2} \mathrm{O} & \mathrm{He}\end{array}$ $\begin{array}{llllllll}\text { total mol \% } & 4.8 & 0 & 16.4 & 37.9 & 15.0 & 11.6 & 11.4\end{array}$ normalized mol \% $5.6 \quad 0 \quad 19.1 \quad 44.2 \quad 17.5 \quad 13.5$

Residue Weight: $0.48 \mathrm{~g}$ of organic phase (loss $=0.64 \mathrm{~g}$ ) and $0.91 \mathrm{~g}$ of aqueous phase left (loss $=0.53 \mathrm{~g}$ )

Residue Composition: (by acid-base titration, Karl-Fisher titration and ${ }^{1} \mathrm{H}$ NMR) Organic Phase $(0.48 \mathrm{~g})$

$\left[\mathrm{H}^{+}\right]: \quad \mathrm{N} / \mathrm{A}^{* *}$

$\left[\mathrm{H}_{2} \mathrm{O}\right]: \quad \mathrm{N} / \mathrm{A}^{* *}$

[TBP]: $\quad 0.56 \mathrm{mmol}$

[DBP]: $\quad 1.00 \mathrm{mmol}$

[MBP]: $\quad 0.56 \mathrm{mmol}$

$\left[\mathrm{BuONO}_{2}\right]: \quad 0.37 \mathrm{mmol}$

[PrCOOH]: $0.72 \mathrm{mmol}$

[EtCOOH]: $0.72 \mathrm{mmol}$

[MeCOOH]: $0.54 \mathrm{mmol}$

Aqueous Phase $(0.91 \mathrm{~g})$

$\left[\mathrm{H}^{+}\right]$:

$3.00 \mathrm{mmol}(4.33 \mathrm{M})$

[EtCOOH]: $0.80 \mathrm{mmol}(1.16 \mathrm{M})$

[MBP]: $\quad 0.43 \mathrm{mmol}(0.62 \mathrm{M})$

[MeCOOH]: $0.96 \mathrm{mmol}(1.39 \mathrm{M})$

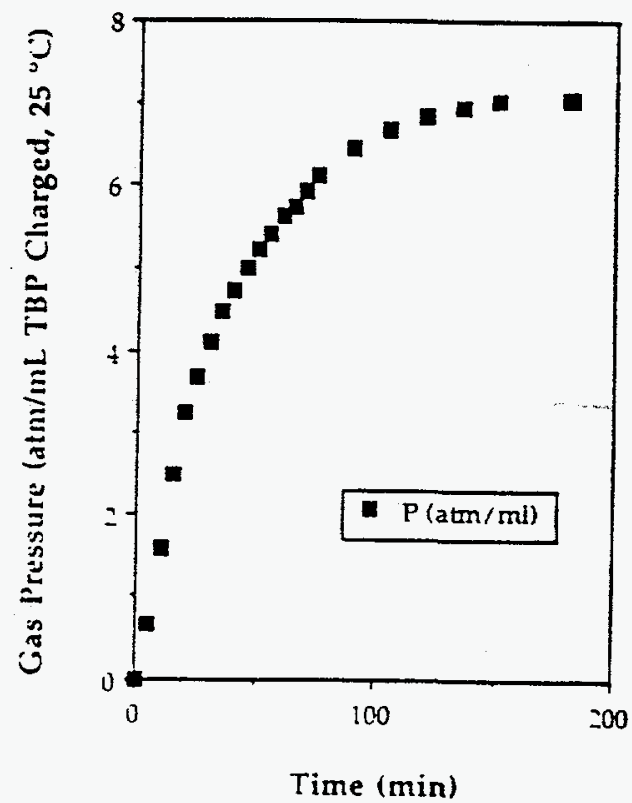

** Not available because of solid residue 
WSRC-RP-95-259

Results of Experiment No. 163 (Const. Volume, Single Phase)

Reaction Conditions:

Bath Temperature:

Sample Volume:

Catalyst:

Before Reaction:

Sample Weight:

$\left[\mathrm{H}^{+}\right]$:

$\left[\mathrm{H}_{2} \mathrm{O}\right]:$

[TBP]:

\section{After Reaction:}

Total Gas Pressure: $\quad 11.0$ atms $\left(25^{\circ} \mathrm{C}, 58 \mathrm{~mL}\right.$ volume)

$\begin{array}{llllllll}\text { Gas Composition: } & \mathrm{N}_{2} & \mathrm{O}_{2} & \mathrm{CO} & \mathrm{CO}_{2} & \mathrm{NO} & \mathrm{N}_{2} \mathrm{O} & \mathrm{He}\end{array}$

$\begin{array}{llllllll}\text { total } \mathrm{mol} \mathrm{\%} & 14.8 & 0 & 24.7 & 38.3 & 0 & 8.7 & 8.1\end{array}$

$\begin{array}{lllllll}\text { normalized mol \% } & 17.1 & 0 & 28.6 & 44.3 & 0 & 10.1\end{array}$

Residue Weight: $4.03 \mathrm{~g}$ (loss $=1.53 \mathrm{~g}$ )

Residue Composition: (by acid-base titration, Karl-Fisher titration and ${ }^{1} \mathrm{H}$ NMR)

$\left[\mathrm{H}^{+}\right]$:

$15.07 \mathrm{mmol}(3.74 \mathrm{M})$

$\left[\mathrm{H}_{2} \mathrm{O}\right]: \quad 19.18 \mathrm{mmol}(4.76 \mathrm{M})$

[TBP]: *

[DBP]: *

[MBP]: *

$\left[\mathrm{BuONO}_{2}\right]:$ *

[PrCOOH]: *

[EtCOOH]: *

[MeCOOH]: *

[HCOOH]: *
Acid concentration: $\quad \in M$

Atmosphere: Helium
$5.56 \mathrm{~g}$

$29.00 \mathrm{mmol}(5.80 \mathrm{M})$

$16.35 \mathrm{mmol}(3.27 \mathrm{M})$

$12.80 \mathrm{mmol}(2.56 \mathrm{M})$ 
Results of Experiment No. 165 (Const. Pressure, Two Phase)

Reaction Conditions:

Bath Temperature: $\quad 130^{\circ} \mathrm{C}$

Sample Volume: $\quad 2.0 \mathrm{~mL}$ of each phase

Acid concentration: $\quad 6 \mathrm{M}$

Distillation/Refluxing $\mathrm{D}$

Atmosphere: Helium

Before Reaction:

Sample Weight: $2.22 \mathrm{~g}$ of organic and $2.81 \mathrm{~g}$ of aqueous phase

$\left[\mathrm{H}^{+}\right]$: $\quad 11.50 \mathrm{mmol}(5.75 \mathrm{M}$, organic) and $30.08 \mathrm{mmol}$ (15.04 M, aqueous)

$\left[\mathrm{H}_{2} \mathrm{O}\right]: \quad 3.76 \mathrm{mmol}(1.88 \mathrm{M}$, organic) $\quad$ [TBP]: $\quad 5.32 \mathrm{mmol}(2.66 \mathrm{M})$

\section{After Reaction:}

Total Gas Volume: $\quad 166 \mathrm{~mL}\left(25^{\circ} \mathrm{C}, 1 \mathrm{~atm}\right)$

$\begin{array}{llllllll}\text { Gas Composition: } & \mathrm{N}_{2} & \mathrm{O}_{2} & \mathrm{CO} & \mathrm{CO}_{2} & \mathrm{NO} & \mathrm{N}_{2} \mathrm{O} & \mathrm{He}\end{array}$

$\begin{array}{llllllll}\text { total mol \% } & 25.5 & 0 & 5.8 & 23.4 & 3.9 & 1.3 & 39.0\end{array}$

normalized mol \% $42.6 \quad 0 \quad \begin{array}{lllll}9.7 & 39.1 & 6.5 & 2.2\end{array}$

Residue Weight: $1.42 \mathrm{~g}$ of organic phase (loss $=0.80 \mathrm{~g}$ ) and no aqueous phase Residue Composition: (by acid-base titration, Karl-Fisher titration and ${ }^{1} \mathrm{H}$ NMR) . $\left[\mathrm{H}^{+}\right]$: $\quad 5.89 \mathrm{mmol}(4.15 \mathrm{M})$

$\left[\mathrm{H}_{2} \mathrm{O}\right]: \quad 0.92 \mathrm{mmol}(0.65 \mathrm{M})$

[TBP]: $\quad 2.10 \mathrm{mmol}(1.48 \mathrm{M})$

[DBP]: $\quad 2.55 \mathrm{mmol}(1.80 \mathrm{M})$

[MBP]: $\quad 0.67 \mathrm{mmol}(0.47 \mathrm{M})$

$\left[\mathrm{BuONO}{ }_{2}\right]: \quad 0.16 \mathrm{mmol}(0.11 \mathrm{M})$

[EtCOOH]: $0.10 \mathrm{mmol}(0.07 \mathrm{M})$

[MeCOOH]: $0.08 \mathrm{mmol}(0.06 \mathrm{M})$

[HCOOH]: $\quad 0.02 \mathrm{mmol}(0.01 \mathrm{M})$

Condensate Composition:

Aqueous Phase (2.70 g)

$\left[\mathrm{H}^{+}\right]$:

25.94 $\mathrm{mmol}(12.68 \mathrm{M})$

[EtCOOH]: $1.04 \mathrm{mmol}(0.51 \mathrm{M})$

[MeCOOH]: $0.59 \mathrm{mmol}(0.29 \mathrm{M})$

Organic Phase $(0.22 \mathrm{~g})$

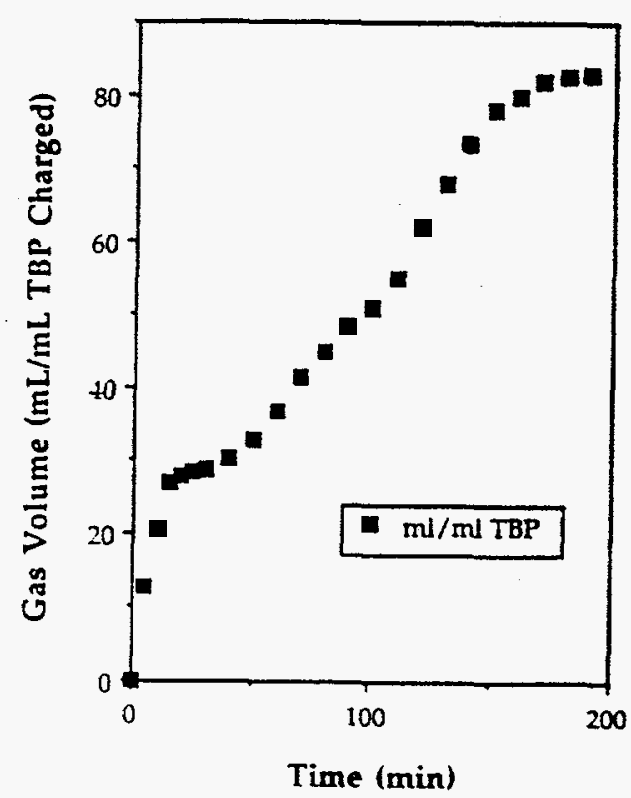

[BuONO 2 ]: $\quad 1.85 \mathrm{mmol}(8.65 \mathrm{M})$

\section{Material Balance}

Carbon loss from residue $=2.74 \mathrm{mmol}$ Nitrogen loss in residue $=13.46 \mathrm{mmol}$
Carbon present in gas $=3.31 \mathrm{mmol}$ Nitrogen present in gas $=6.53 \mathrm{mmol}$ 
Results of Experiment No. 166 (Const. Volume, Single Phase)

Reaction Conditions:

Bath Temperature:

Sample Volume:

$\mathrm{Zr}^{4+}$ :

\section{Before Reaction:}

Sample Weight:

$\left[\mathrm{H}^{+}\right]$:

$\left[\mathrm{H}_{2} \mathrm{O}\right]$ :

[TBP]: $150^{\circ} \mathrm{C}$

$5 \mathrm{ml}$

$\mathrm{Zr}^{4+}$
Acid concentration:

Atmosphere:
$6 \mathrm{M}$

Helium

\section{After Reaction:}

Total Gas Pressure: $\quad 10.5$ atms $\left(25^{\circ} \mathrm{C}, 58 \mathrm{~mL}\right.$ volume $)$

$\begin{array}{llllllll}\text { Gas Composition: } & \mathrm{N}_{2} & \mathrm{O}_{2} & \mathrm{CO} & \mathrm{CO}_{2} & \mathrm{NO} & \mathrm{N}_{2} \mathrm{O} & \mathrm{He}\end{array}$

$\begin{array}{llllllll}\text { total mol \% } & 14.8 & 0 & 24.3 & 36.7 & 0 & 8.5 & 10.1 \\ \text { normalized mol \% } & 17.6 & 0 & 28.8 & 43.5 & 0 & 10.3 & \end{array}$

Residue Weight: $\quad 3.95 \mathrm{~g}$ (loss $=1.60 \mathrm{~g}$ )

Residue Composition: (by acid-base titration, Karl-Fisher titration and ${ }^{1} \mathrm{H}$ NMR)

$\left[\mathrm{H}^{+}\right]$:

$15.21 \mathrm{mmol}(3.85 \mathrm{M})$

$\left[\mathrm{H}_{2} \mathrm{O}\right]: \quad \quad \quad 18.05 \mathrm{mmol}(4.57 \mathrm{M})$

[TBP]: *

[DBP]: *

[MBP]: *

$\left[\mathrm{BuONO}_{2}\right]: *$

[PrCOOH]: *

[EtCOOH]: *

$[\mathrm{MeCOOH}]: *$

$[\mathrm{HCOOH}]: *$

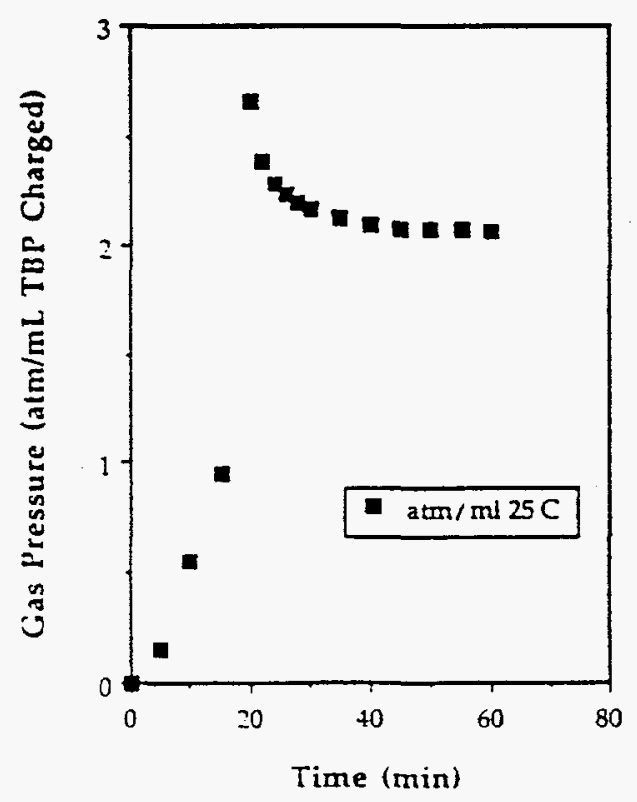

* The presence of paramagnetic materia-tnade ${ }^{1} \mathrm{H}$ NMR analysis unavailable 
WSRC-RP-95-259

\section{APPENDICES B-E}

\section{EFFECT OF ACID CONCENTRATION}

Appendices B through E provide graphical summaries of the effects of acid concentrations for reactions of TBP at a bath temperature of $150^{\circ} \mathrm{C}$. Appendix B summarizes the data for single phase, constant pressure, distillation experiments (Expts \# 136 and 137), while Appendix C provides data for single phase, constant volume conditions (Expts \# 134 and 135). Similar data for two phase constant pressure (Expts \# 151 and 152), and two phase constant volume (Expts \# 156 and 157) conditions are given in Appendices D and E, respectively. 
Fig. B-1 Effect of Acid Concentration $\left(\mathrm{SP}, \mathrm{CP}, \mathrm{D}, 150^{\circ} \mathrm{C}\right)$

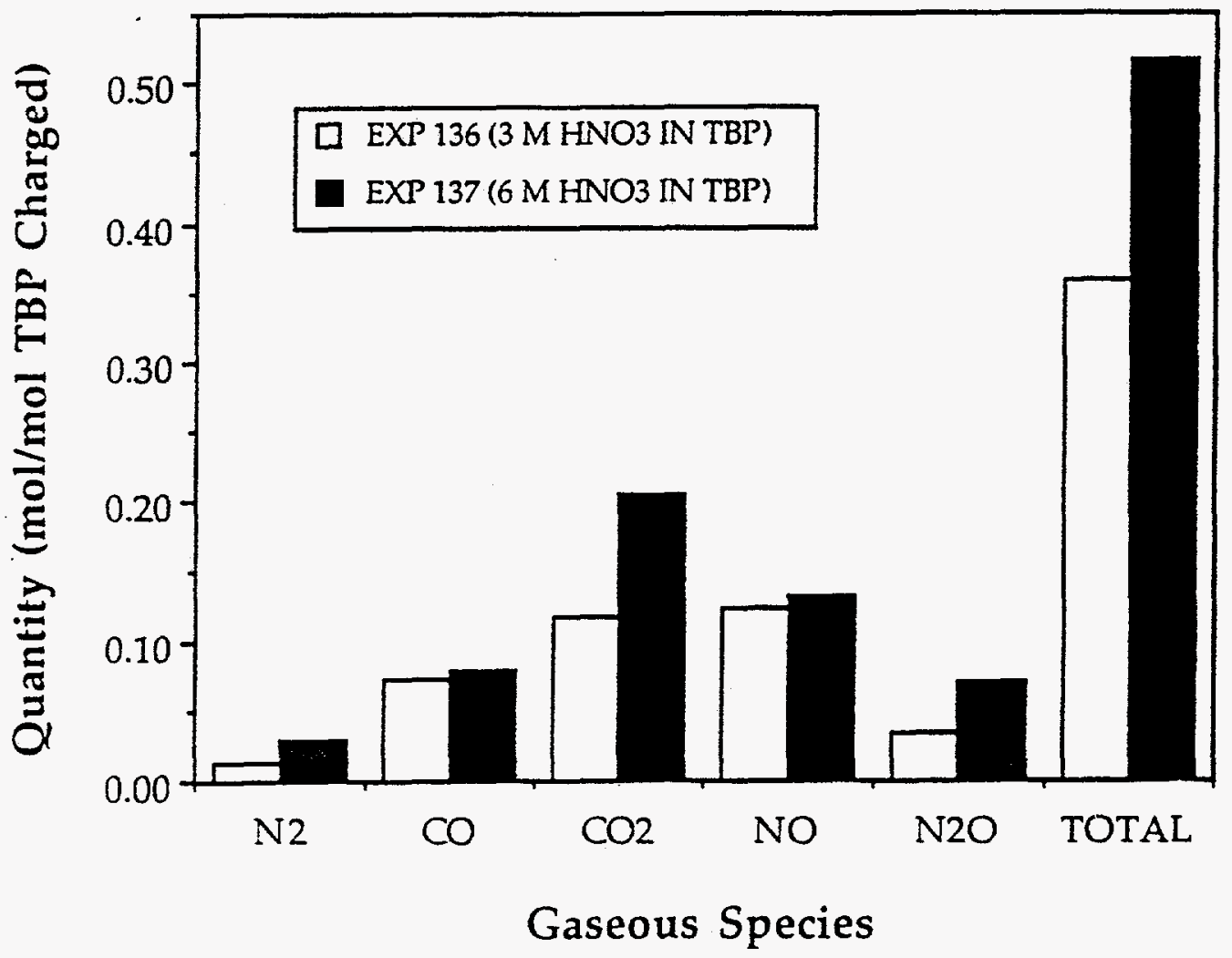


Fig. B-2 Effect of Acid Concentration (SP,CP,D, $\left.150^{\circ} \mathrm{C}\right)$

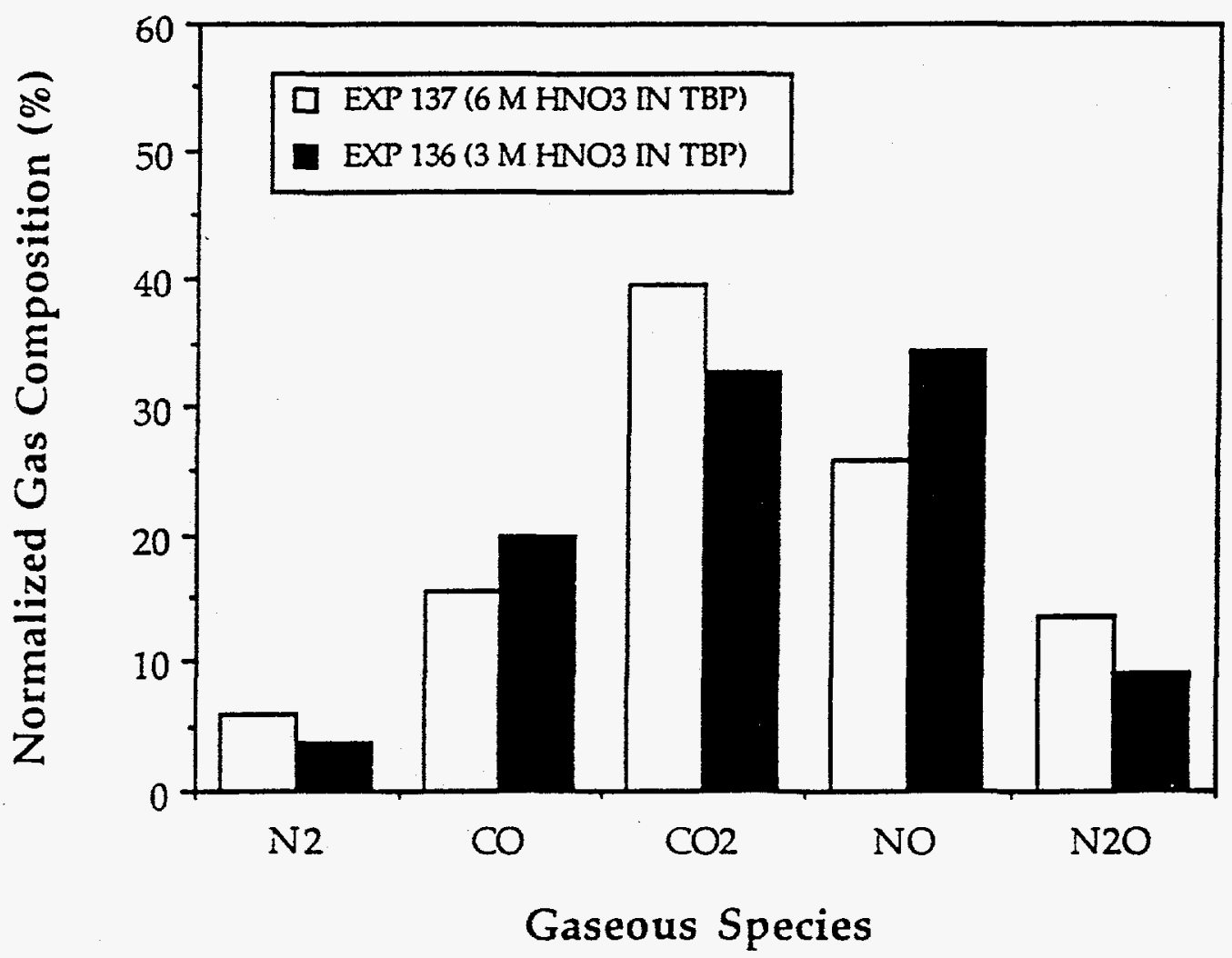


WSRC-RP-95-259

Fig. B-3 Effect of Acid Concentration (SP,CP,D,150 ${ }^{\circ} \mathrm{C}$ )

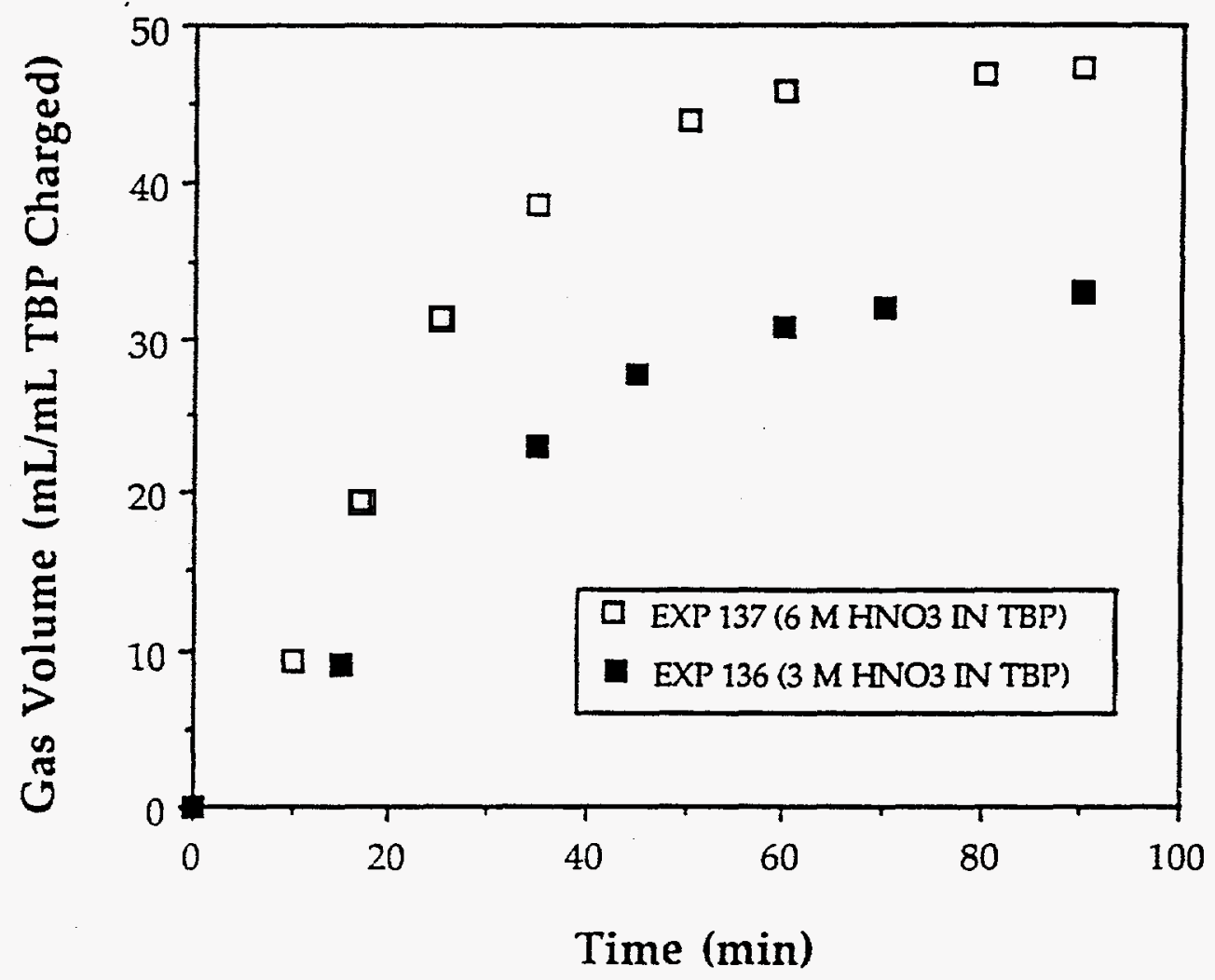


Fig. B-i Effect of Acid Concentration (SP,CP,D, $\left.150^{\circ} \mathrm{C}\right)$

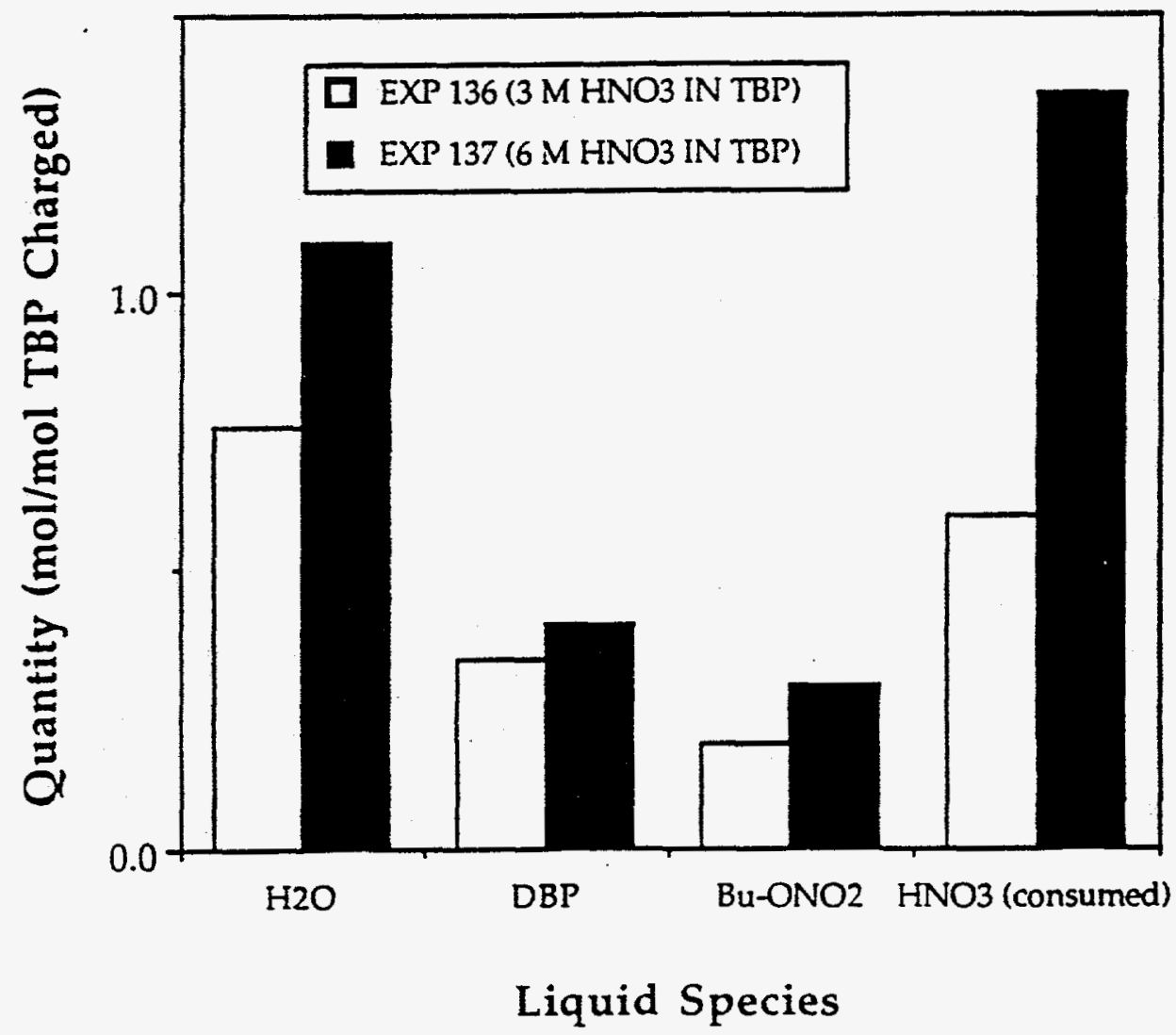


WSRC-RP-95-259

Fig. C-1 Effect ox Aciả Concentration $\left(\mathrm{SP}, \mathrm{CV}, 150^{\circ} \mathrm{C}\right)$

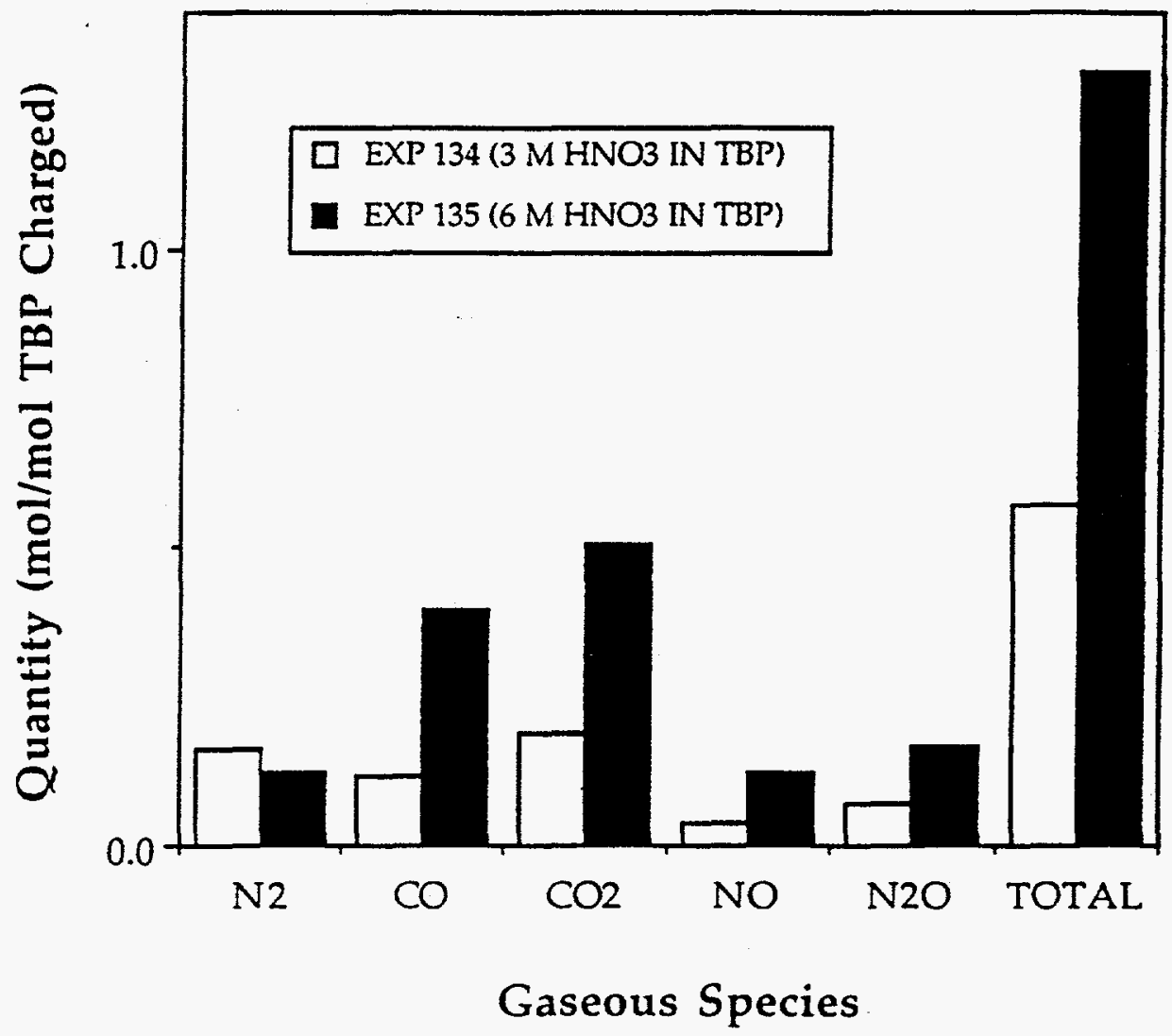


Fig. C-2 Effect of Acid Côncentration (SP,CV, $150^{\circ} \mathrm{C}$ )

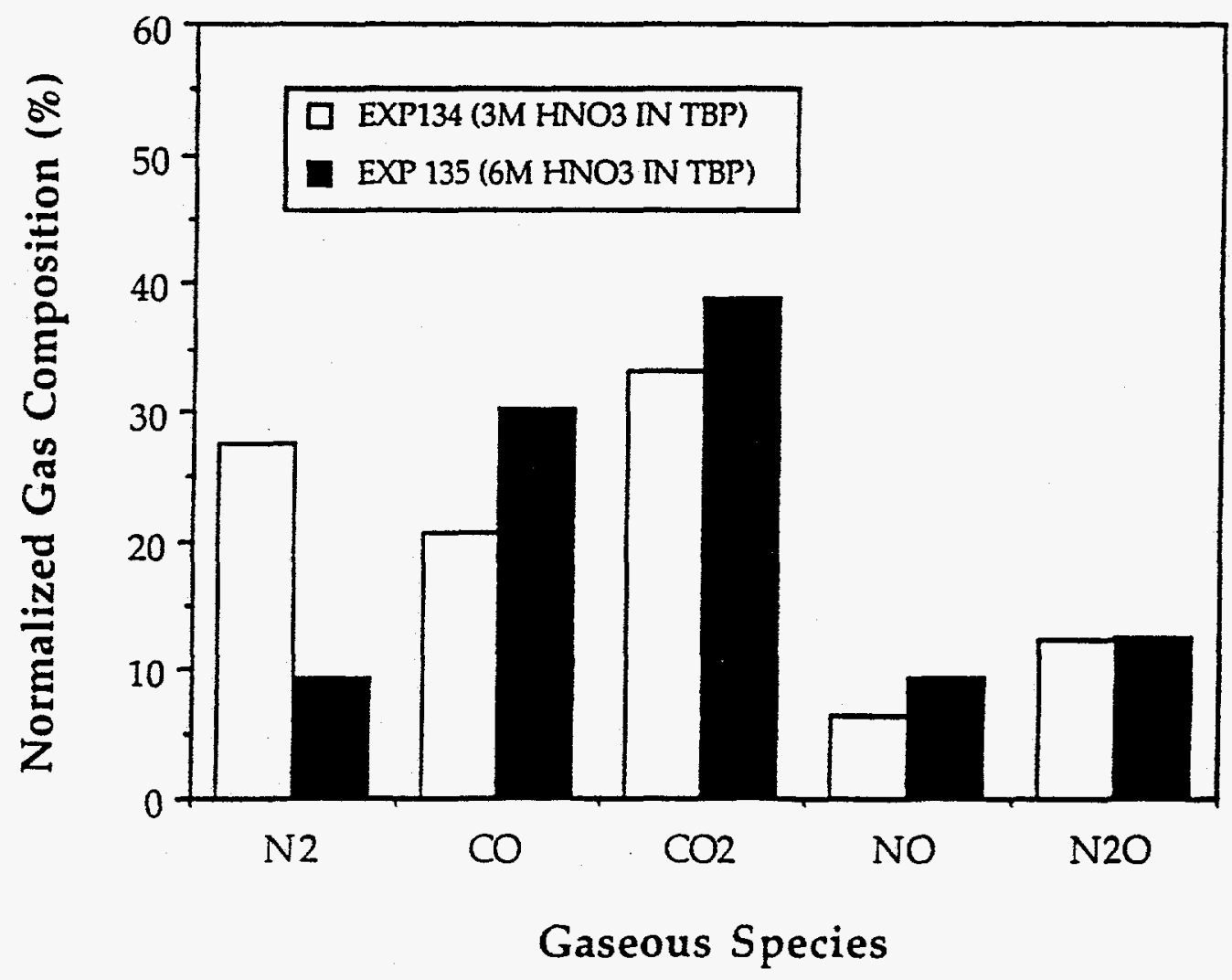


Fig. C-3 Effect of Acid Concentration (SP,CV, $150^{\circ} \mathrm{C}$ )

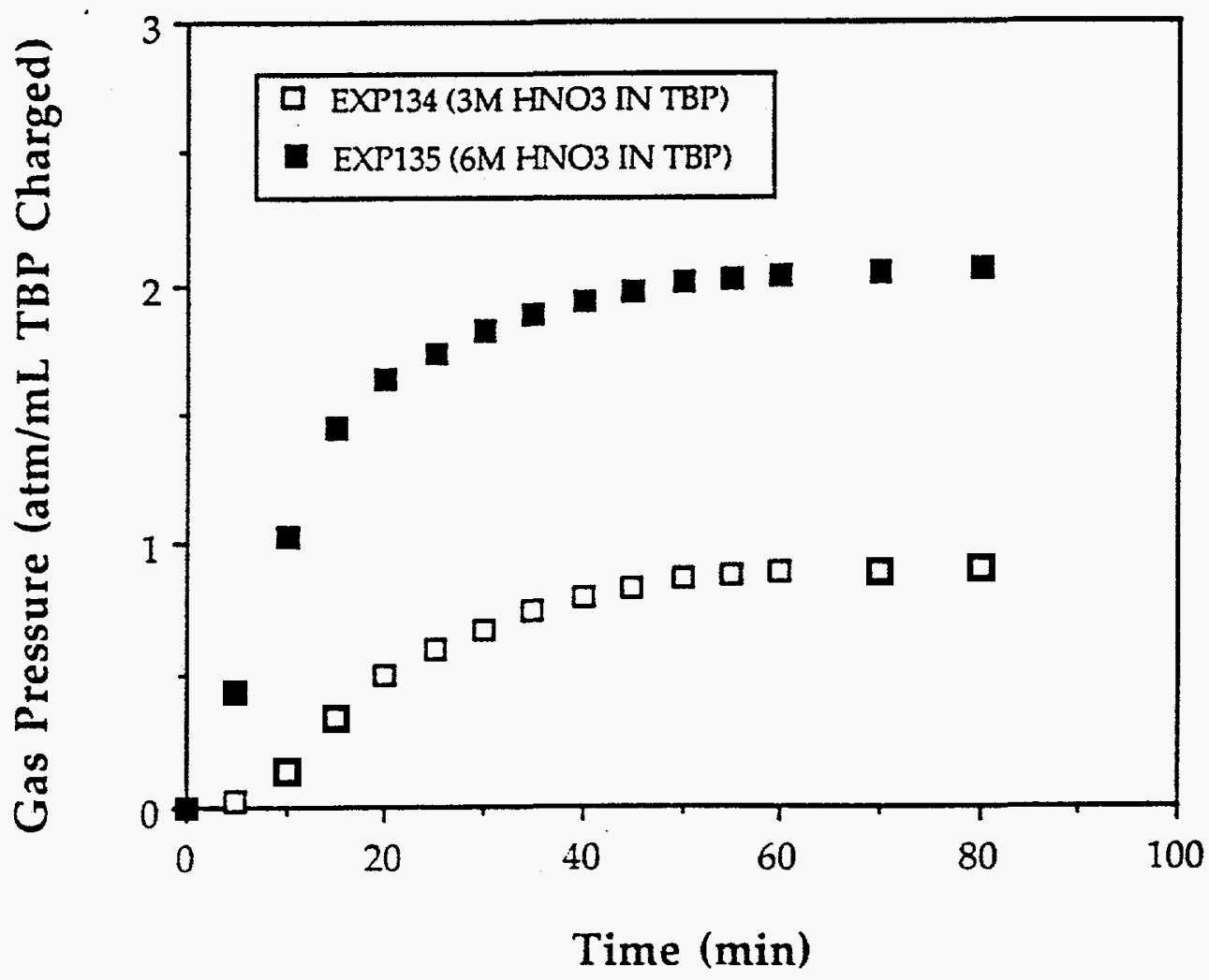


WSRC-RP-95-259

Fig. C-4 Effect of Acid Concentration (SP,CV,150 $\left.{ }^{\circ} \mathrm{C}\right)$

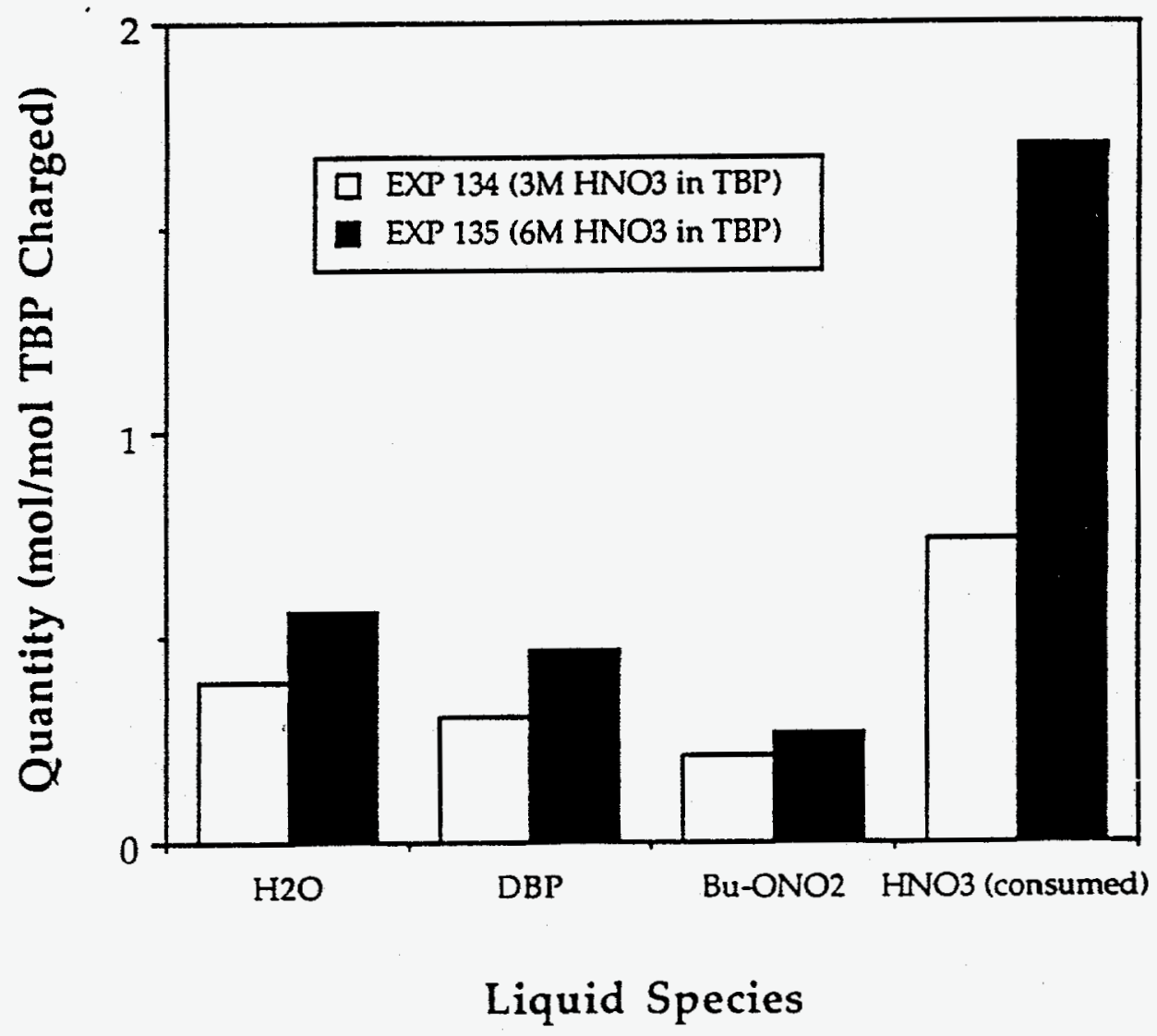


Fig. D-1 Effect of Acid Concentration (TP,CP,D,150 $\left.{ }^{\circ} \mathrm{C}\right)$

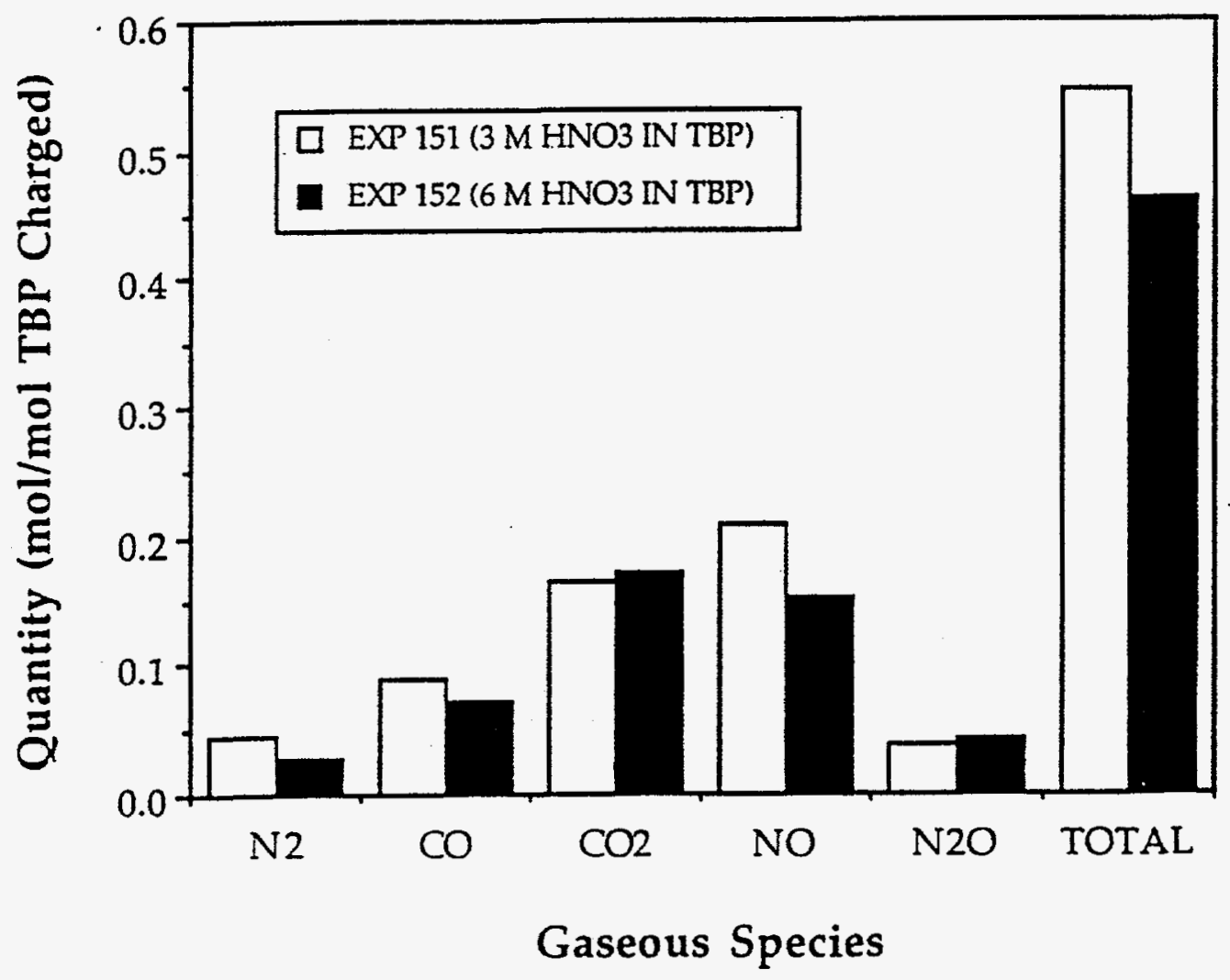


WSRC-RP-95-259

Fig. D-2 Effect of Acid Concentration (TP,CP,D,150 ${ }^{\circ} \mathrm{C}$ )

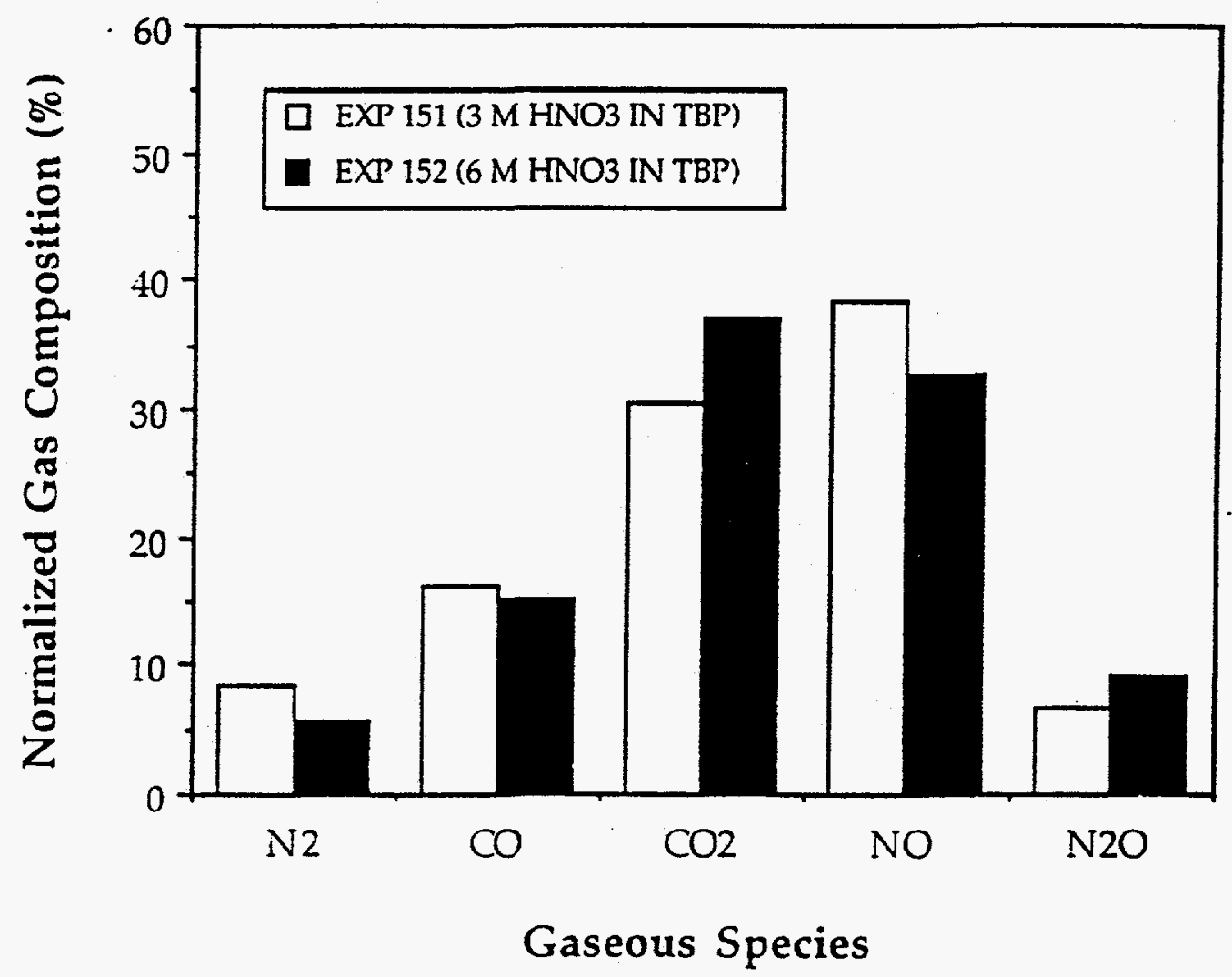


Fig. D-3 Effect of Acid Concentration (TP,CP,D, $\left.150^{\circ} \mathrm{C}\right)$

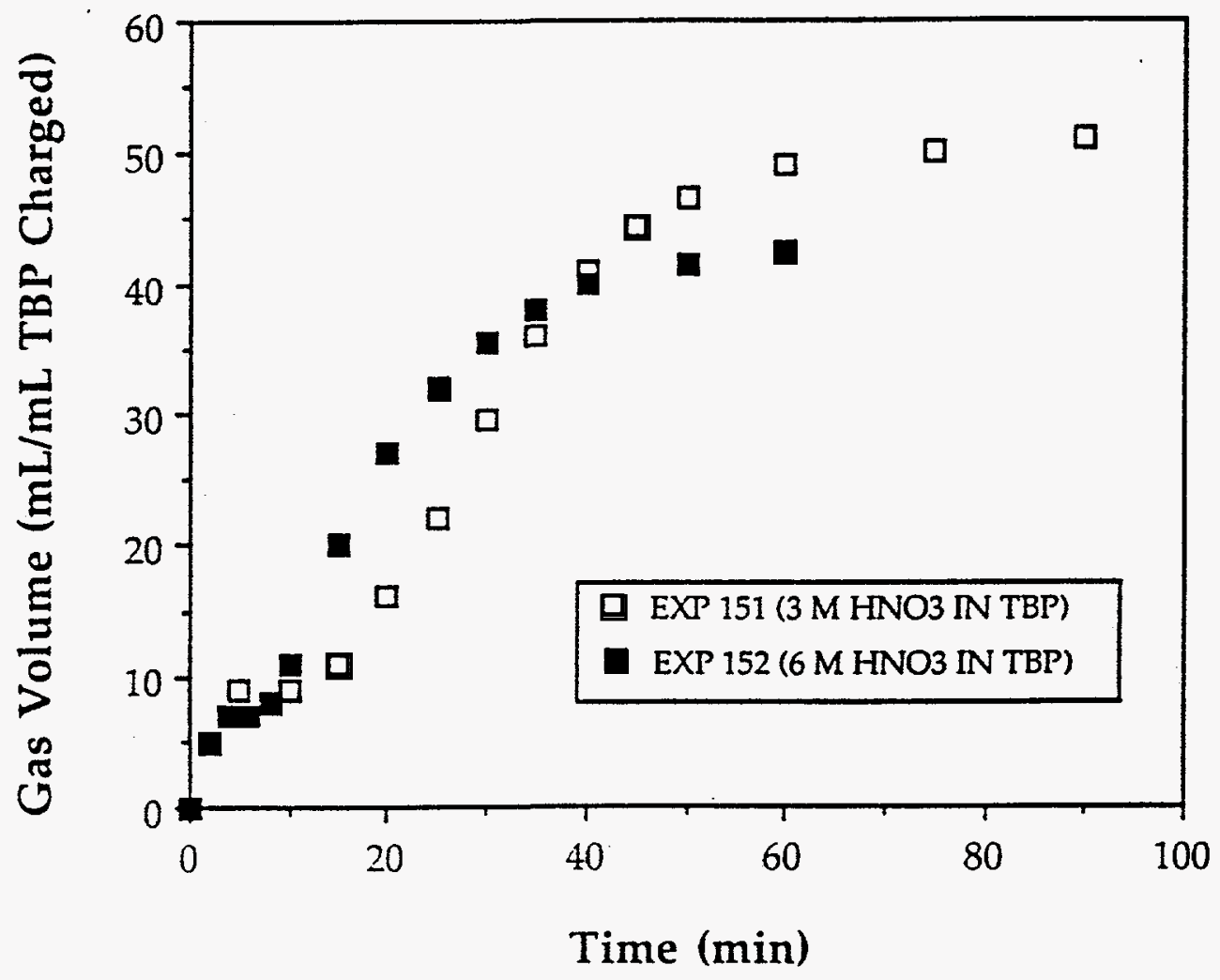


Fig. D-4 Effect of Acid Concentration (TP,CP,D,150 ${ }^{\circ} \mathrm{C}$ )

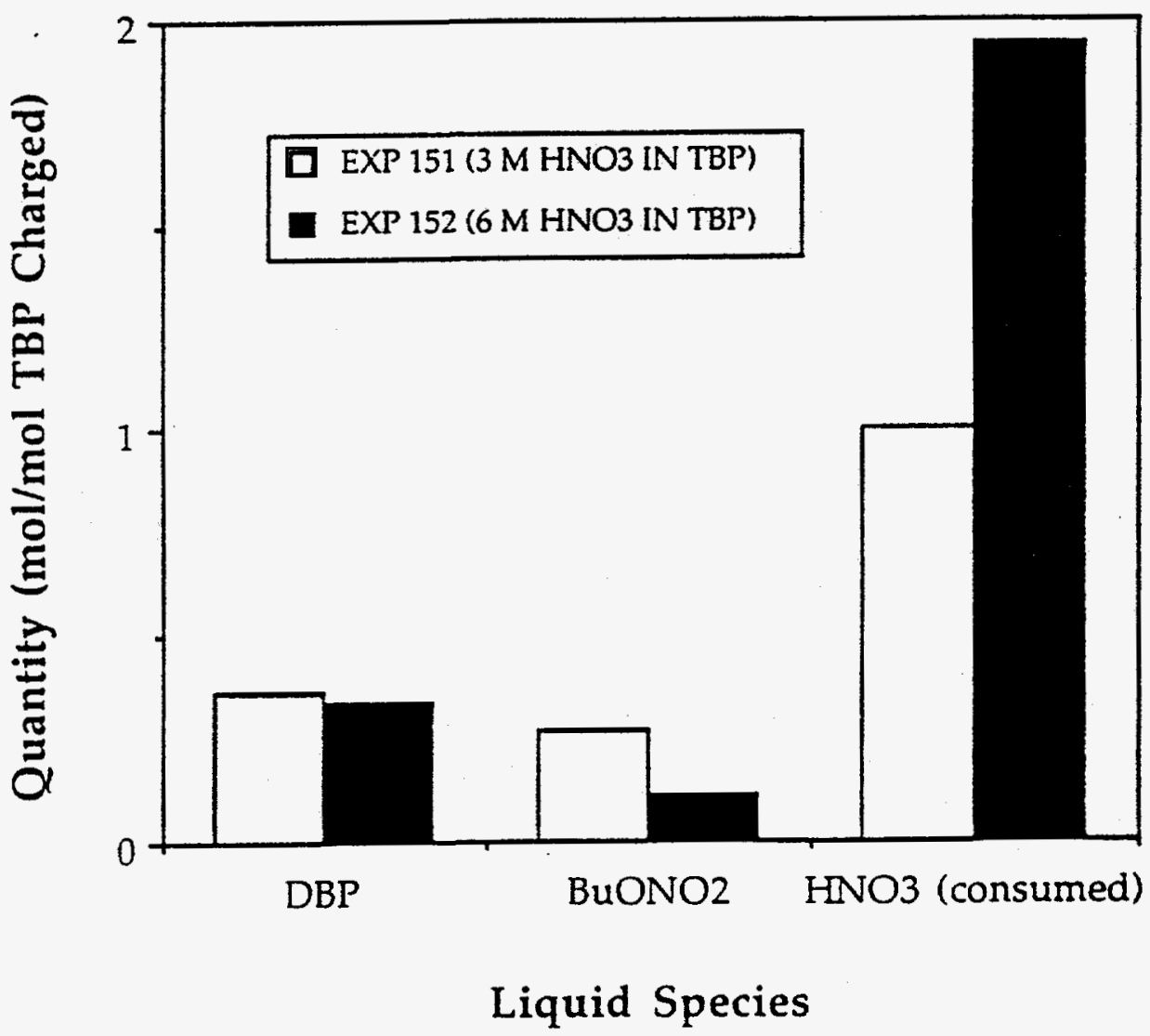



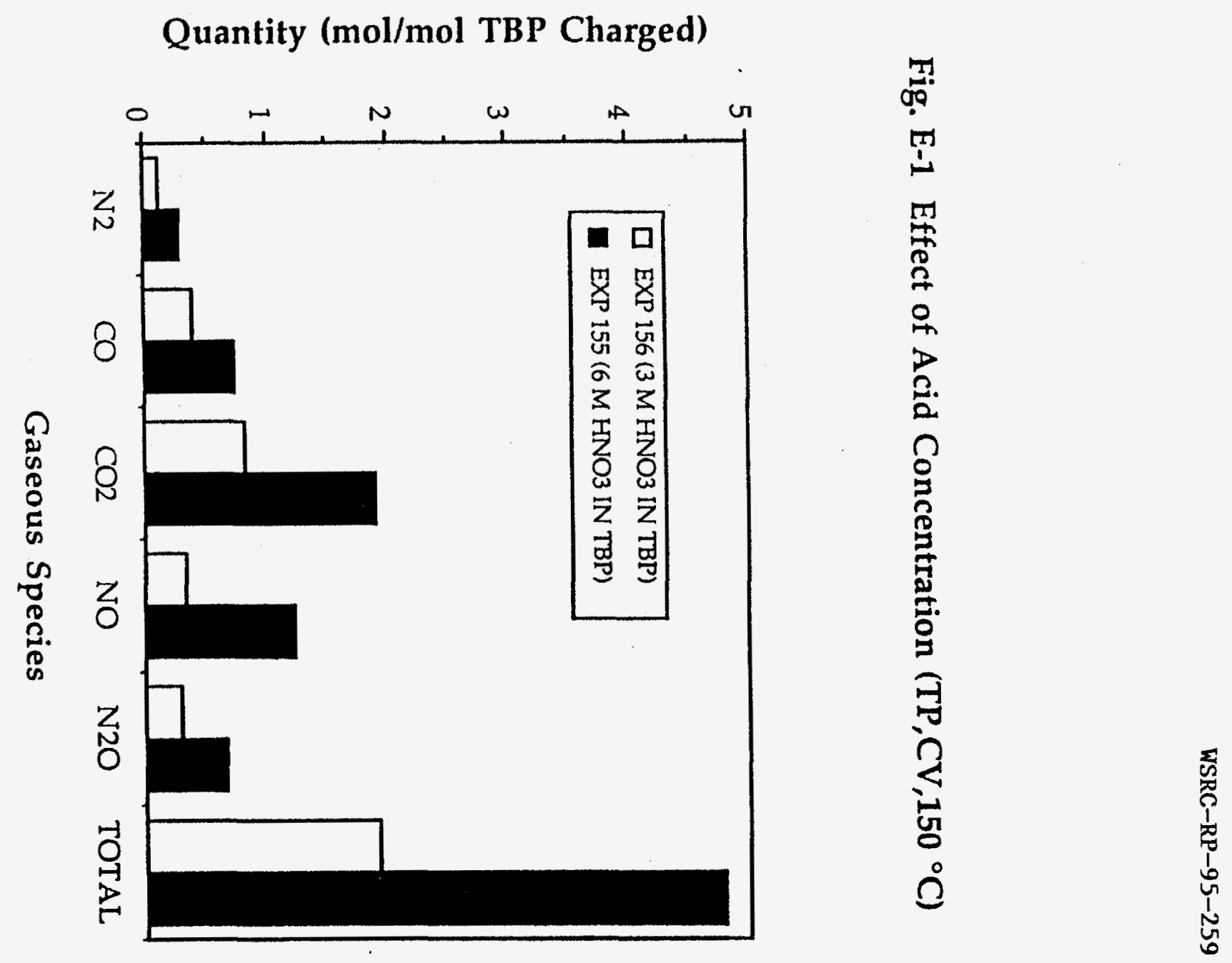
Fig. E-2 Effect of Acid Concentration (TP,CV, $\left.150^{\circ} \mathrm{C}\right)$

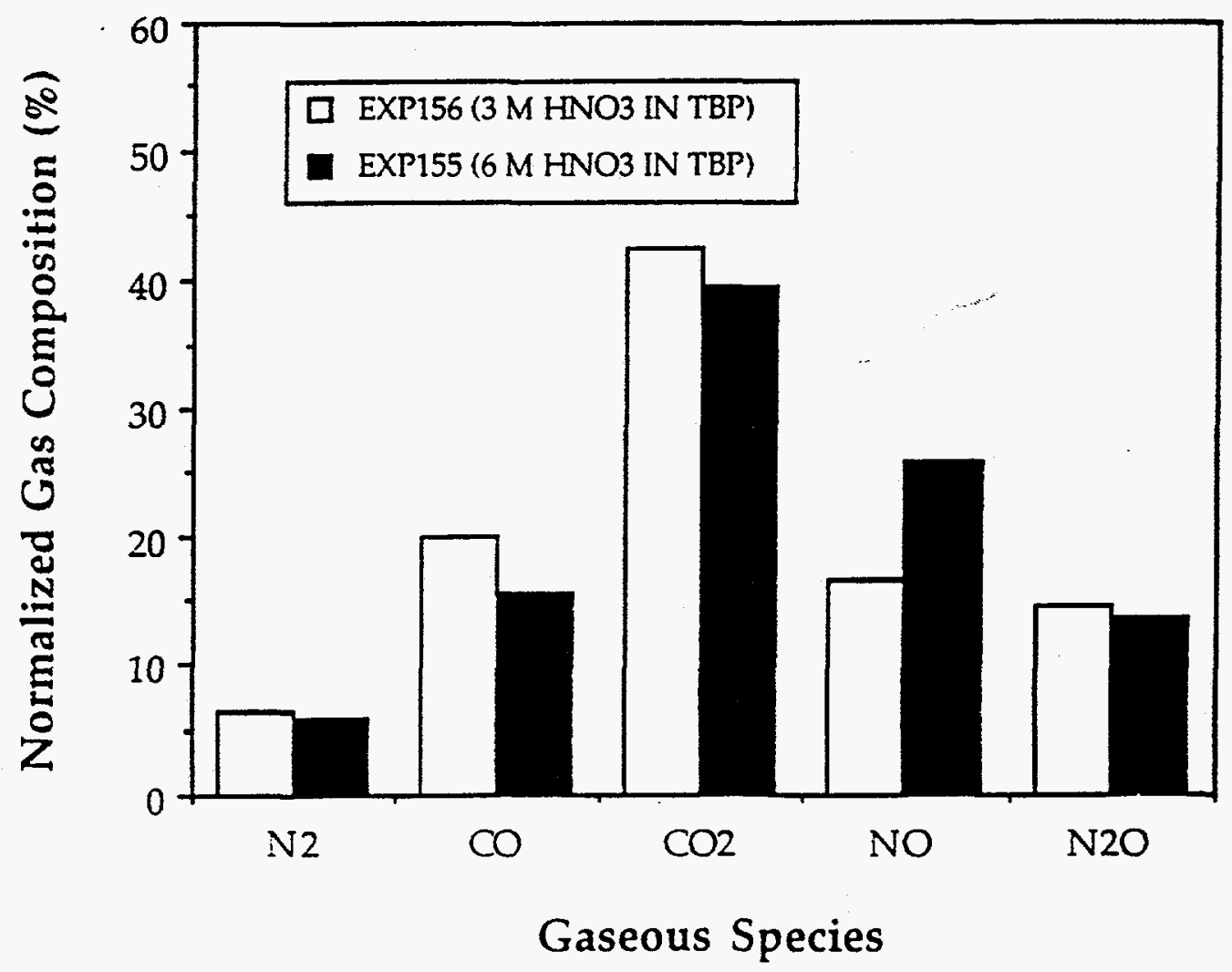


Fig. E-3 Effect of Acid Concentration (TP,CV, $\left.150^{\circ} \mathrm{C}\right)$

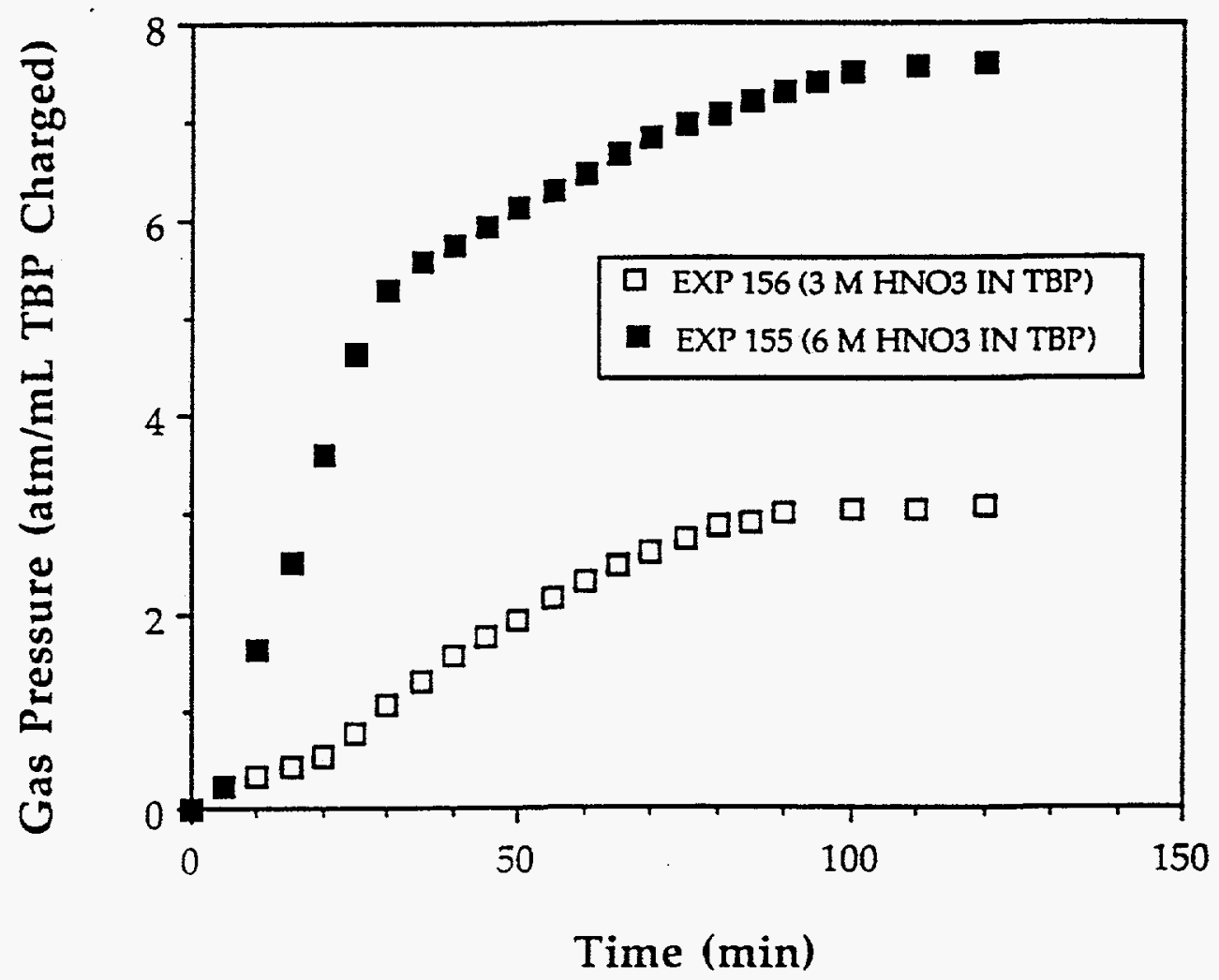


Fig. E-4 Effect of Acid Concentration $\left(\mathrm{SP}, \mathrm{CV}, 150^{\circ} \mathrm{C}\right)$

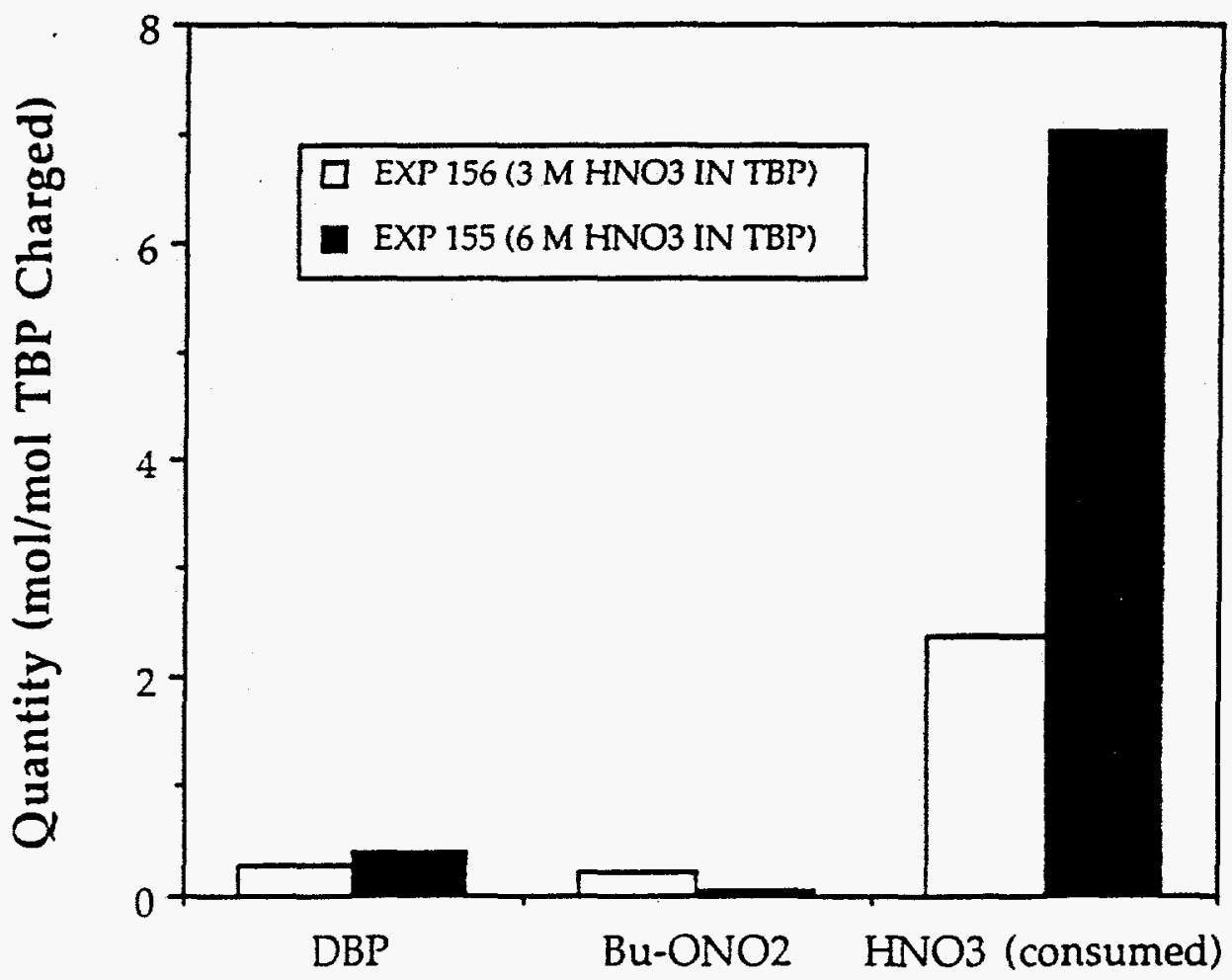

Liquid Species 
WSRC-RP-95-259

\section{APPENDIX F \\ EFFECT OF REDUCED WATER CONTENT}

This appendix provides graphical representation of the effects of reduced water content on single phase reactions of TBP in $3 \mathrm{M}$ nitric acid at $150^{\circ} \mathrm{C}$ with constant pressure distillation conditions (Expts \# 136 and 147A). 
Fig. F-1 Effect of Purging (SP,CP,3M,D, $\left.150^{\circ} \mathrm{C}\right)$

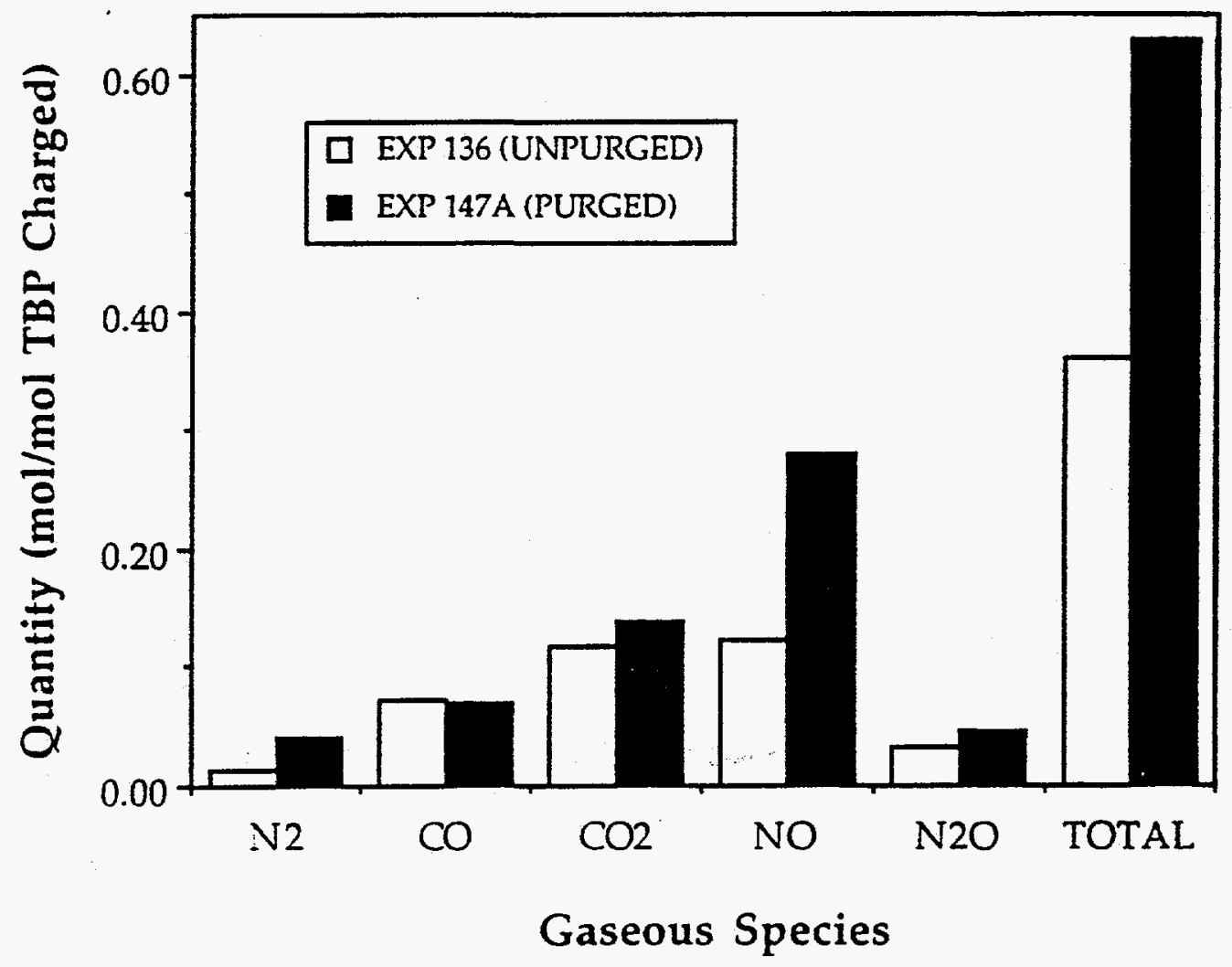


Fig. F-2 Effect of Purging (SP,CP,3M,D,150 $\left.{ }^{\circ} \mathrm{C}\right)$

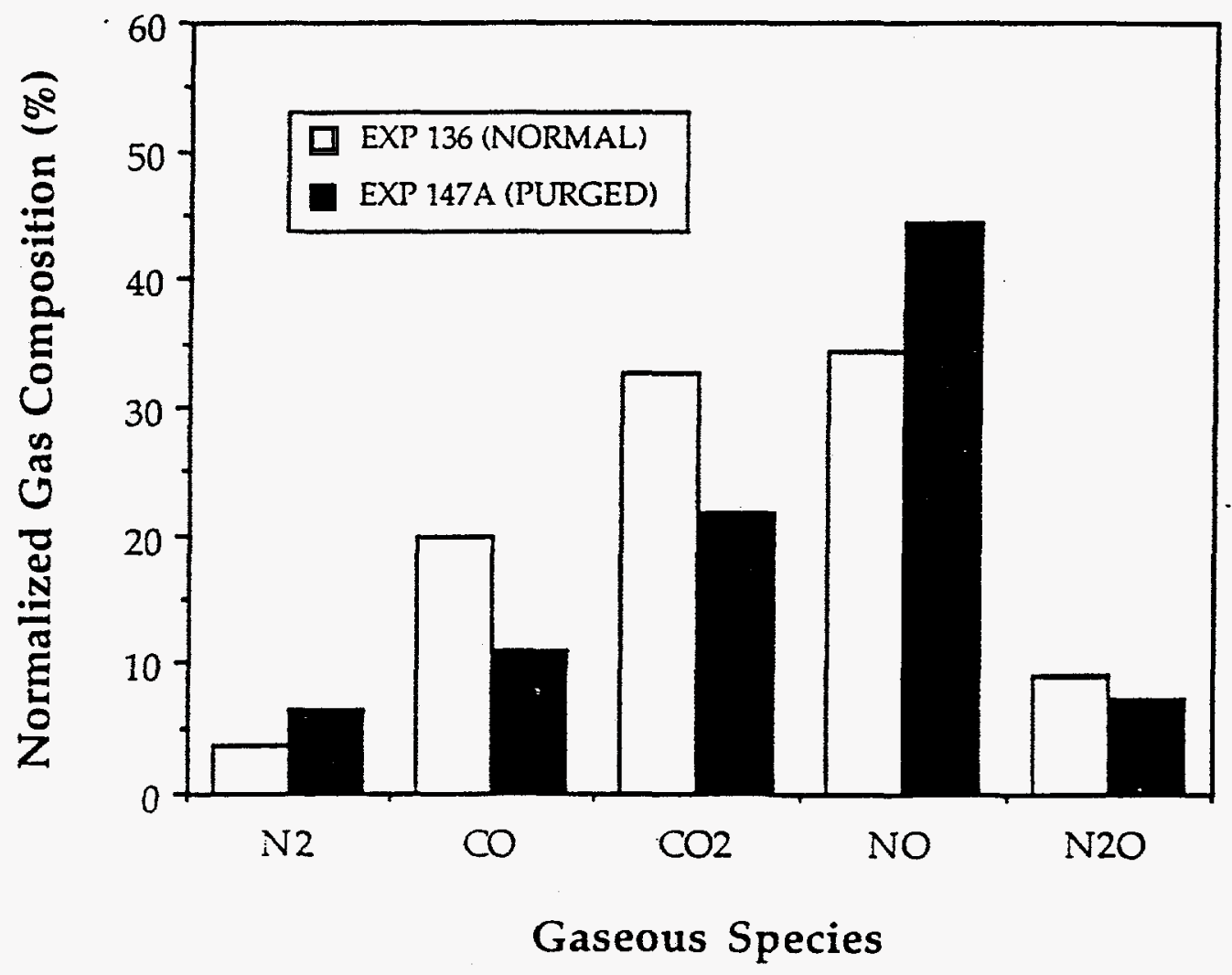


Fig. F-3 Effect of Purging (SP,CP,3M,D, $150^{\circ} \mathrm{C}$ )

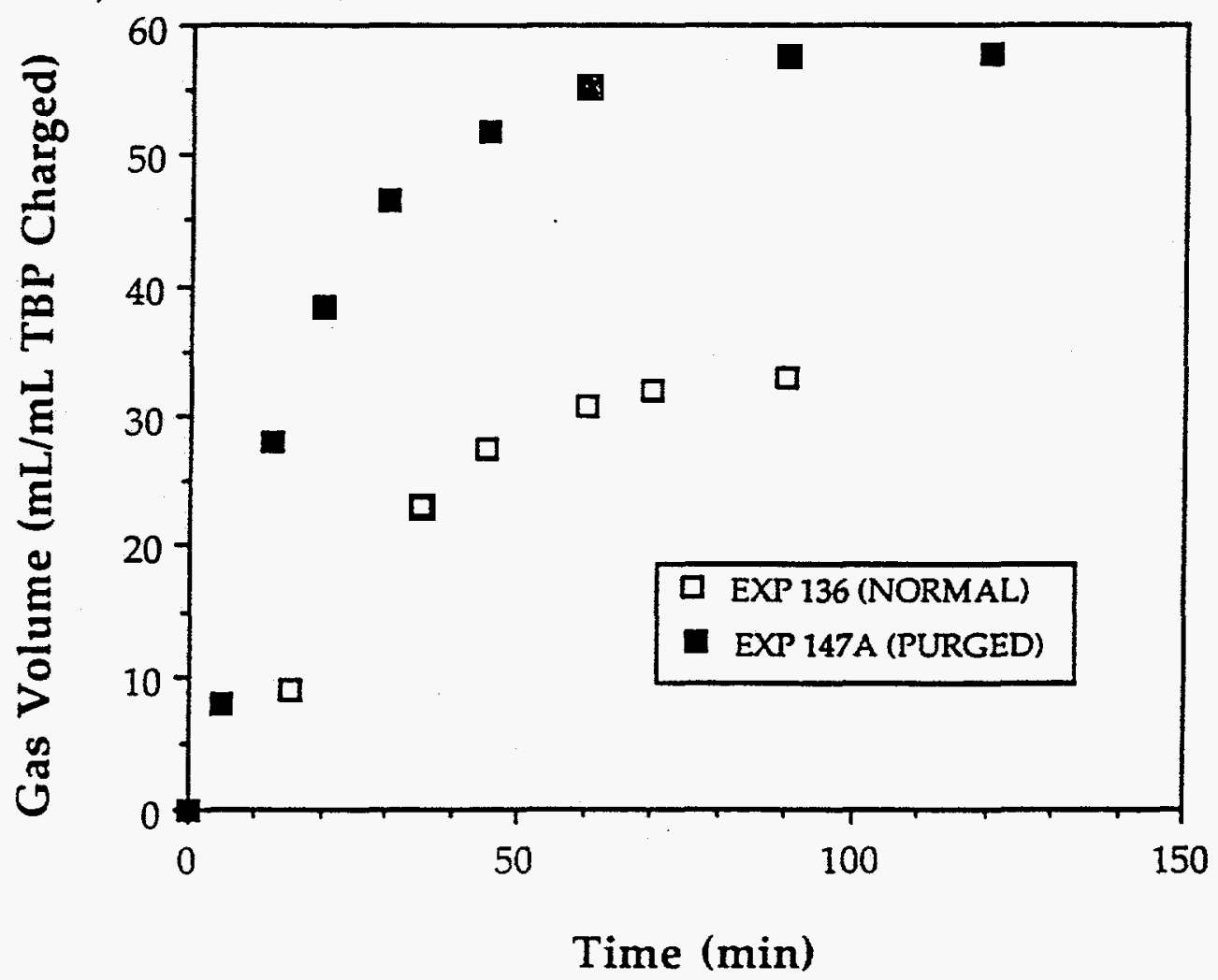


WSRC-RP-95-259

Fig. F-4 Effect of Purging (SP,CP,3M,D,150 ${ }^{\circ} \mathrm{C}$ )

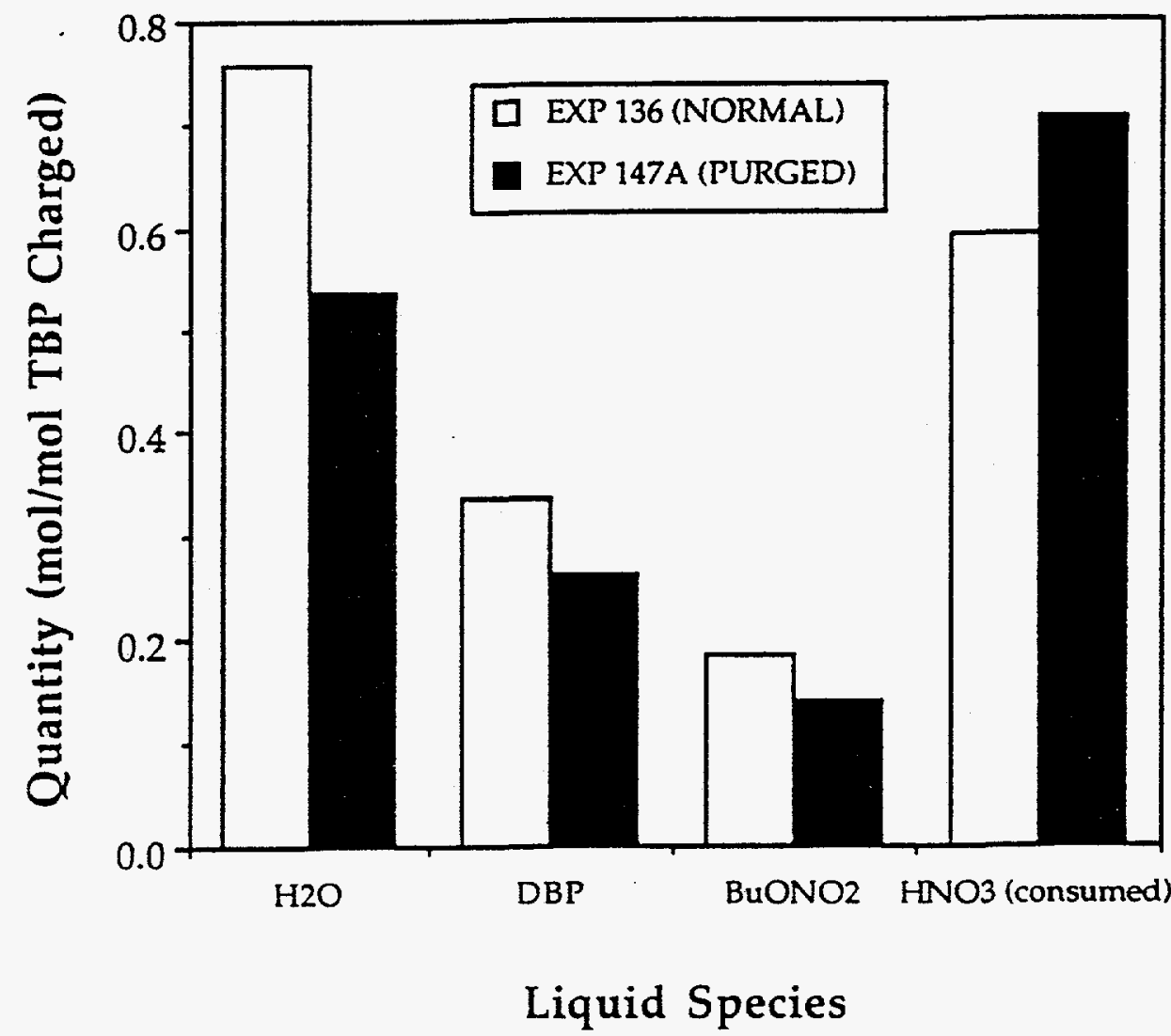


WSRC-RP-95-259

\section{APPENDICES G-L}

\section{EFFECT OF TEMPERATURE}

Appendices G-L provide graphical representation of the effects of temperature for reactions of TBP that is $6 \mathrm{M}$ in nitric acid under the following conditions: single phase, constant pressure, distillation (G); single phase, constant pressure, reflux $(\mathrm{H})$; single phase, constant volume (I); two-phase, constant pressure, distillation (J); two phase, constant pressure, reflux (K); and two phase, constant volume (L). 
Quantity (mol/mol TBP Charged)

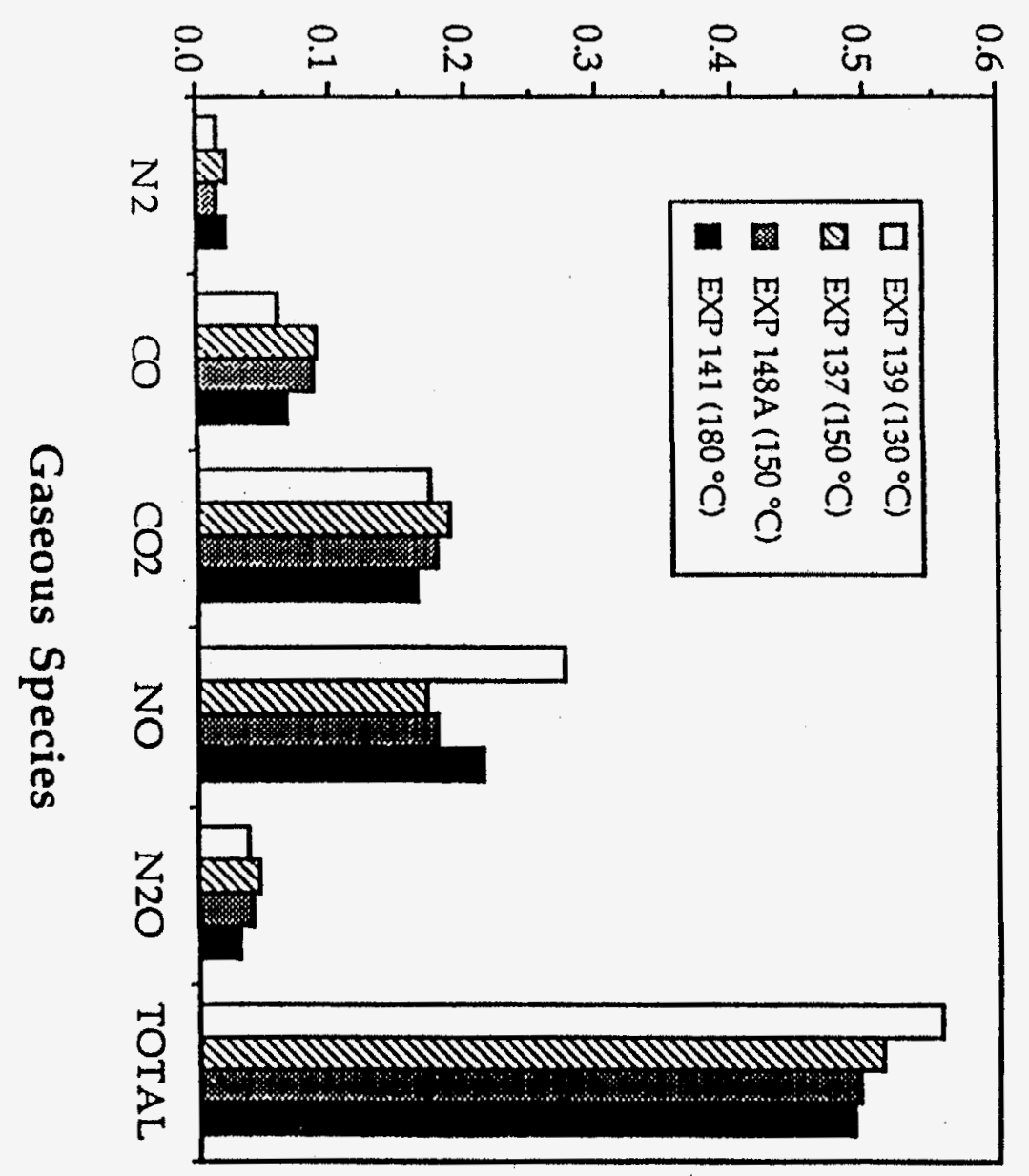

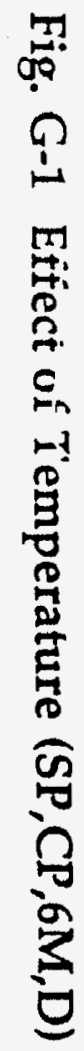


Fig. G-2 Effect of Temperature (SP,CP,6M,D)

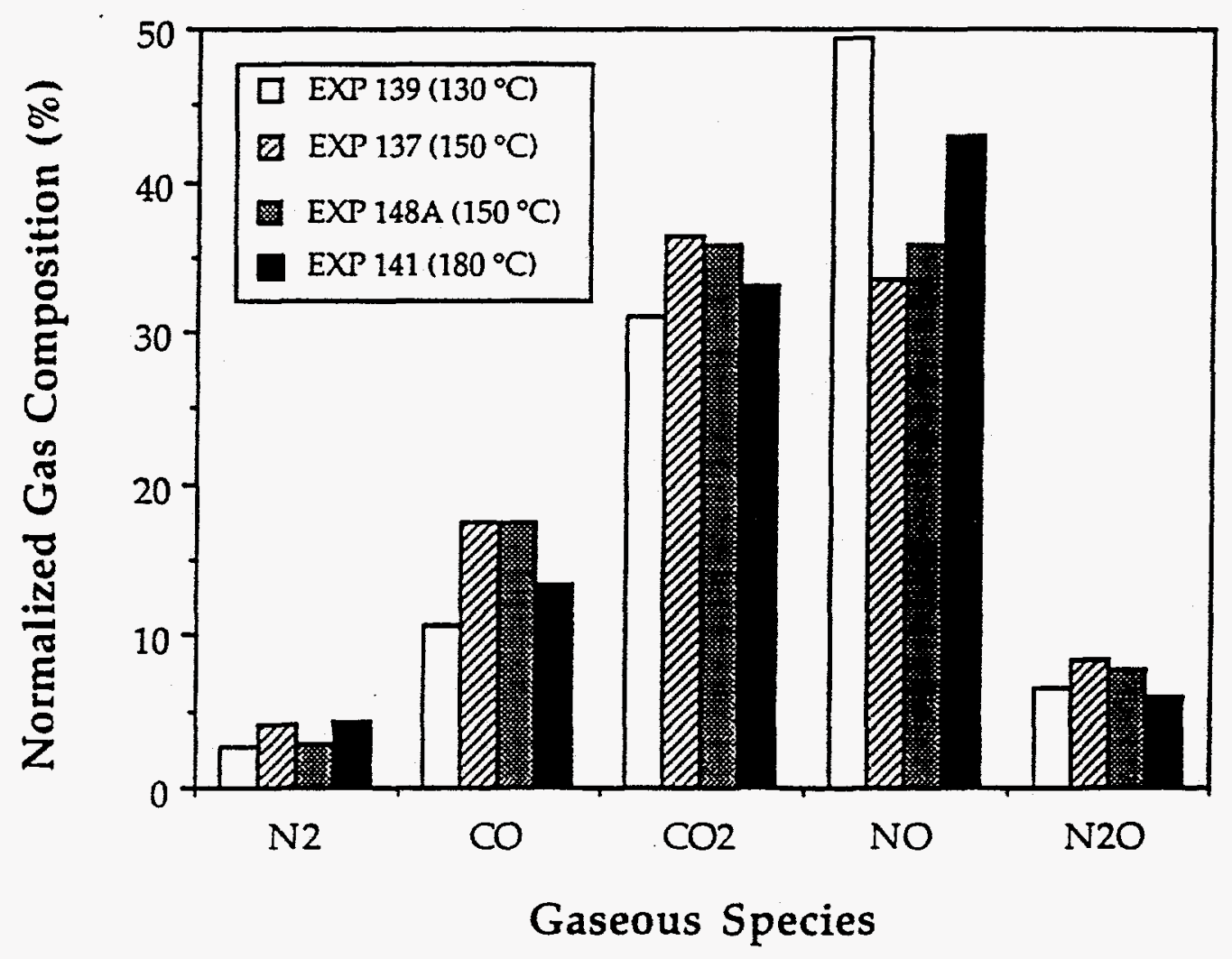


Fig. G-3 Effect of Temperature $(S P, C P, 6 M, D)$

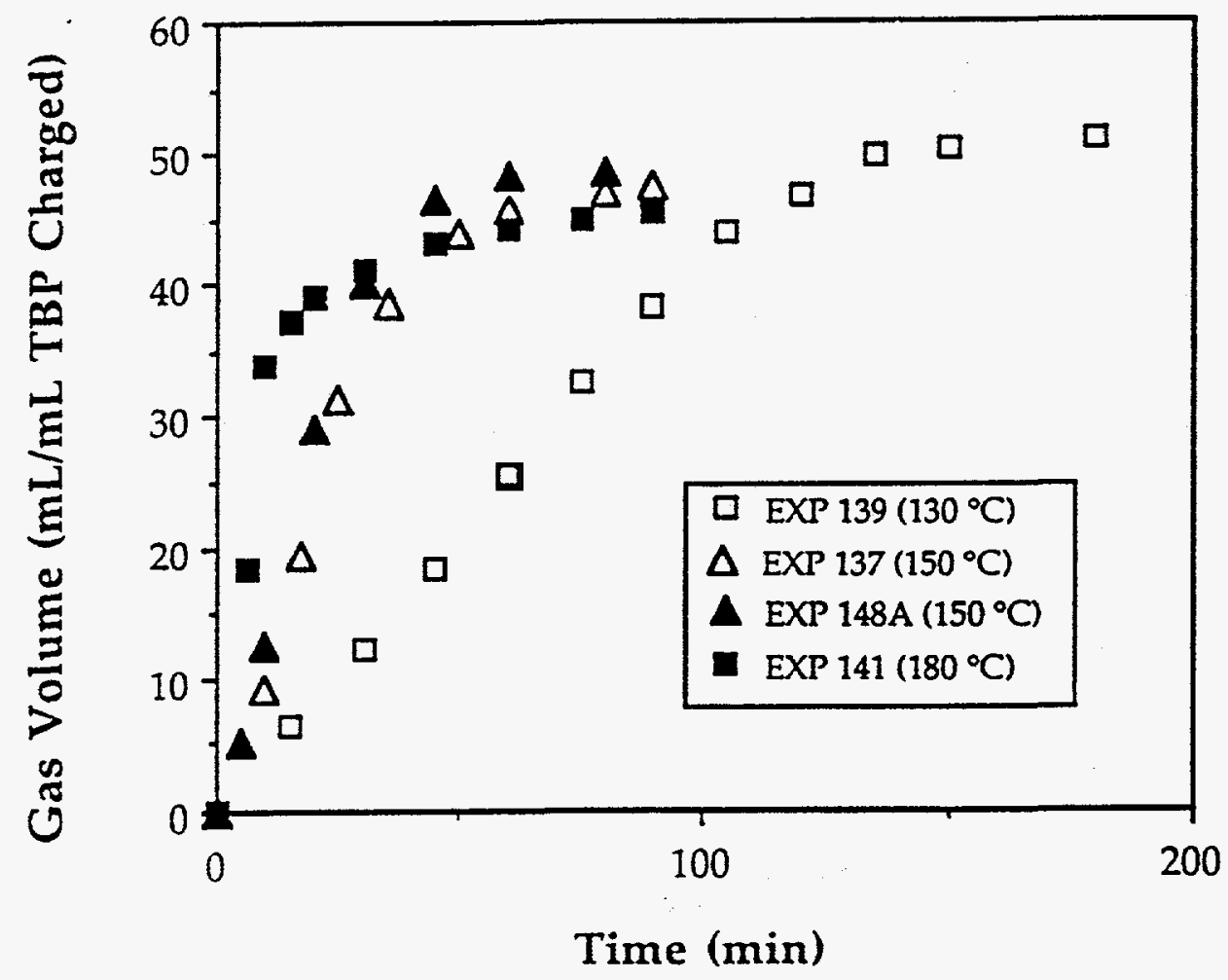


Fig. G-4 Effect of Temperature (SP,CP.óM,D)

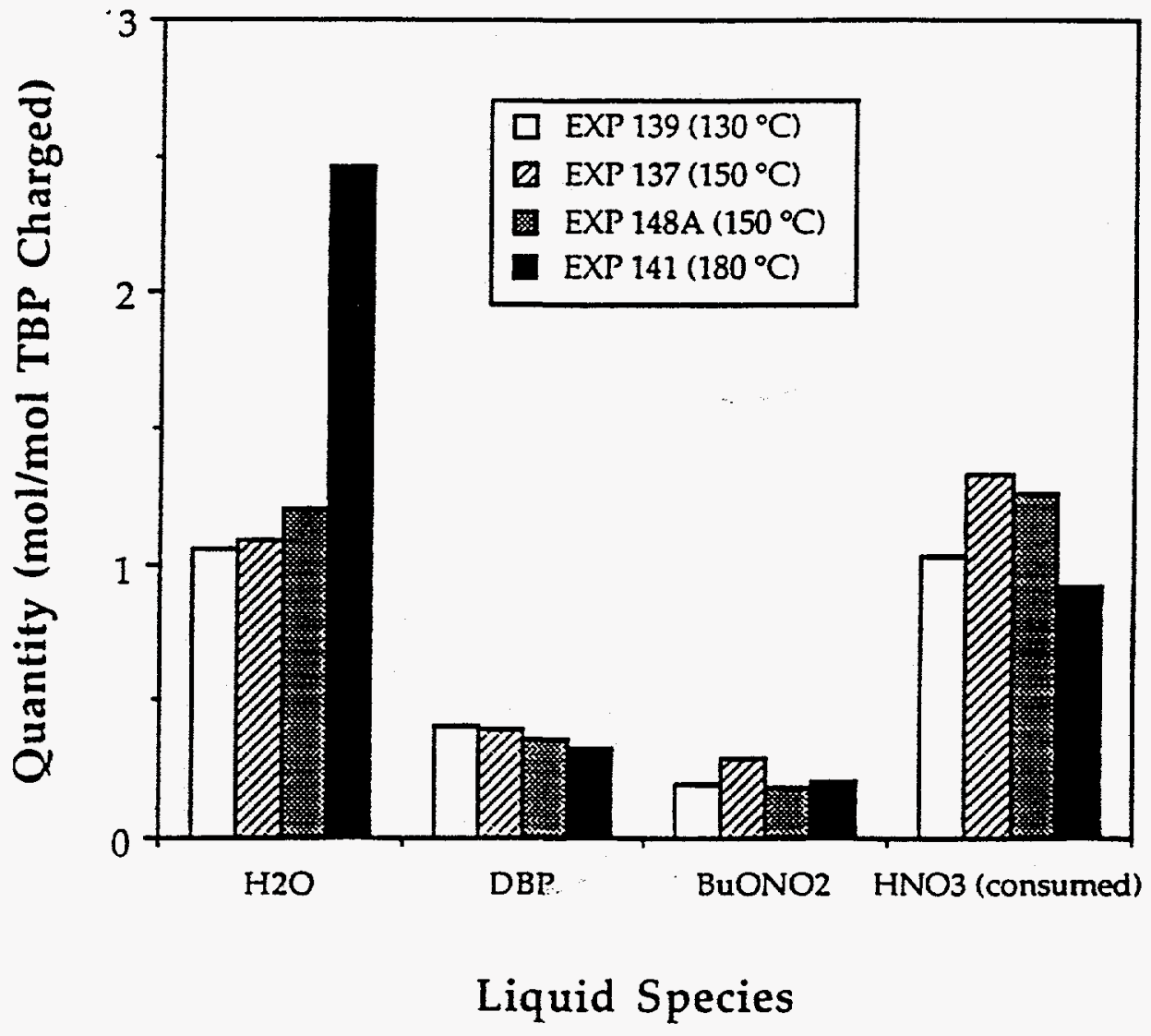


Fig. H-1 Effect of Temperature (SP,CP,6M,R)

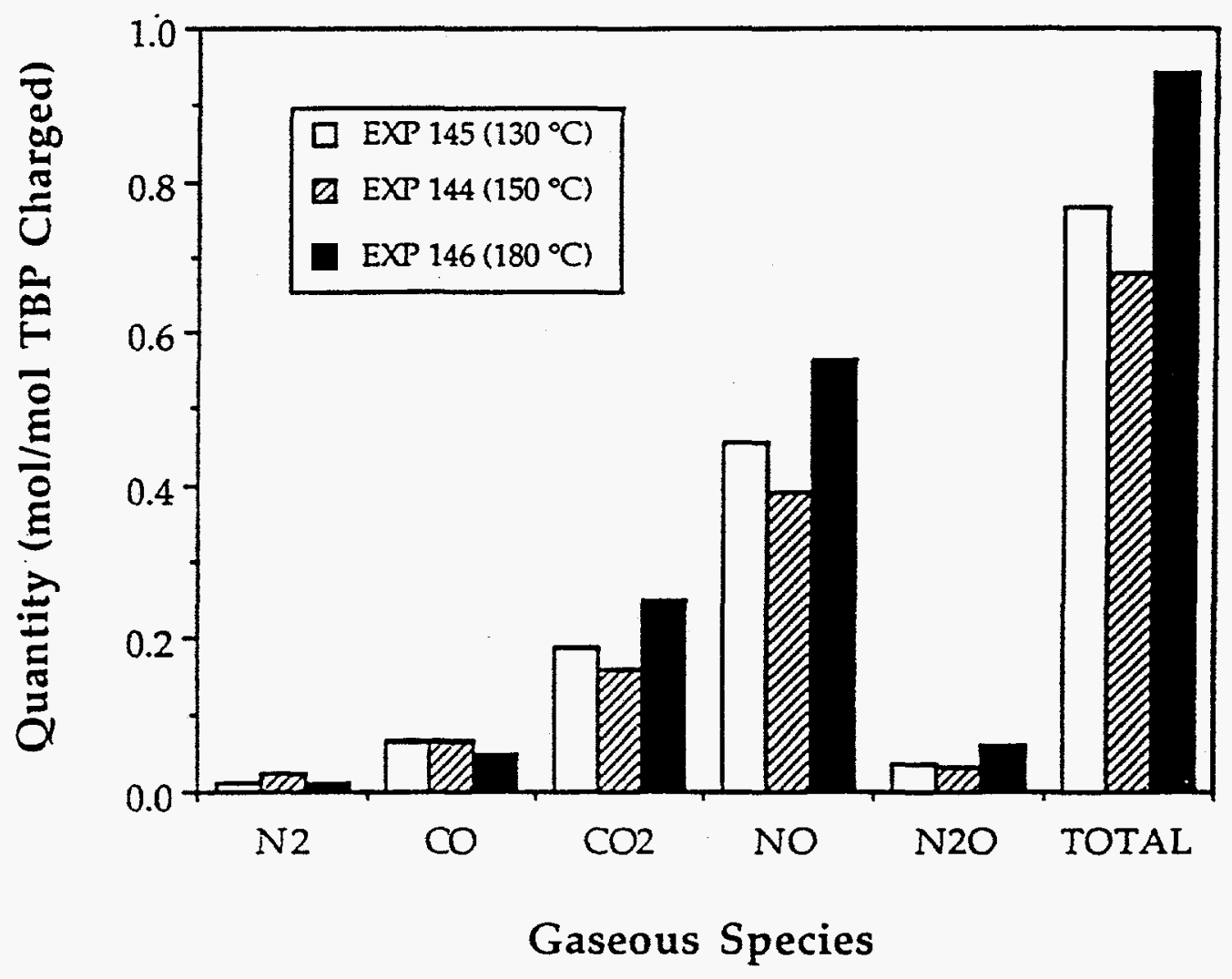


WSRC-RP-95-259

Fig. H-2 Effect of Temperature $(\mathrm{SP}, \mathrm{CP}, 6 \mathrm{M}, \mathrm{R})$

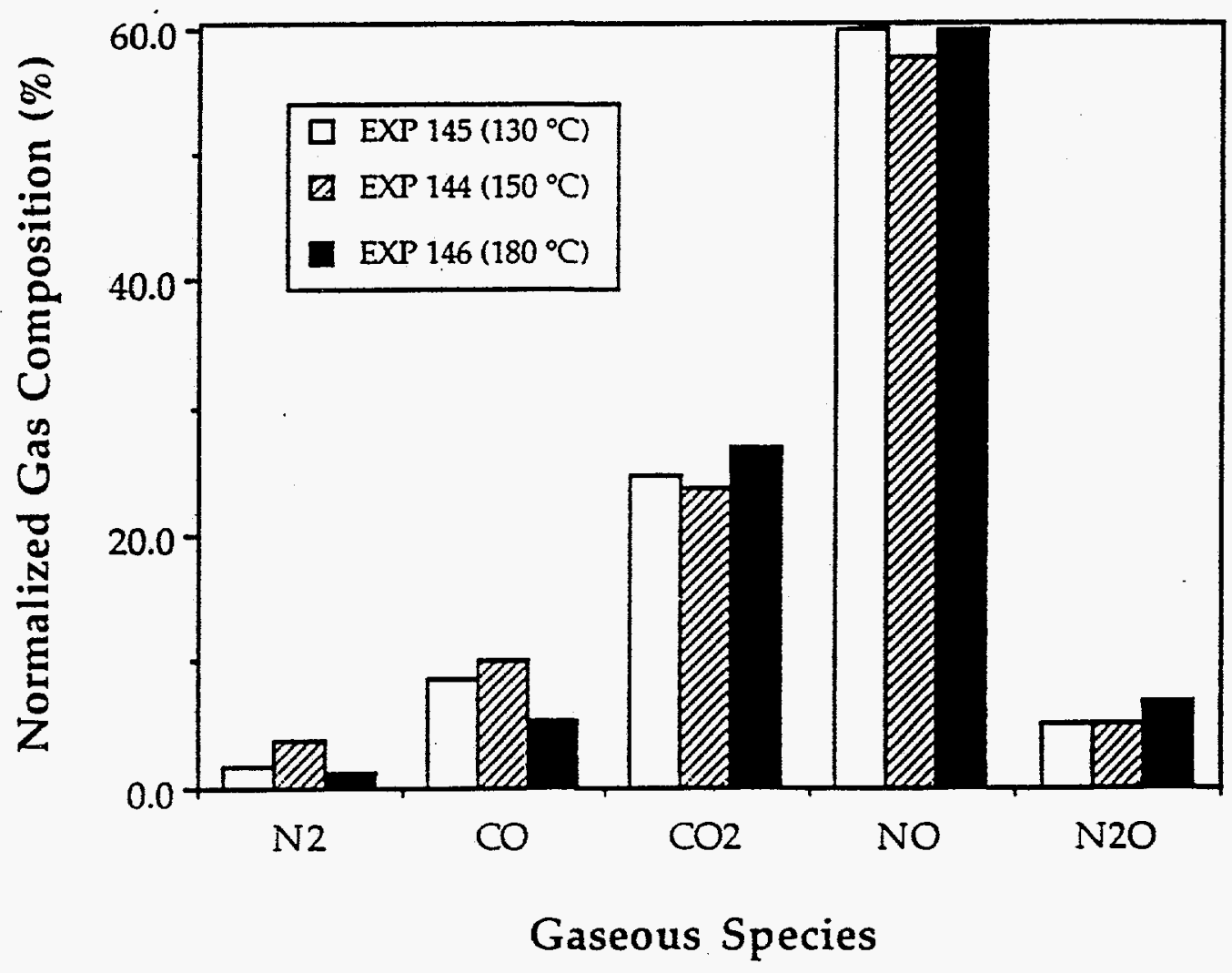


WSRC-RP-95-259

Fig. H-3 Effect of Temperature (SP,CP,6M,R)

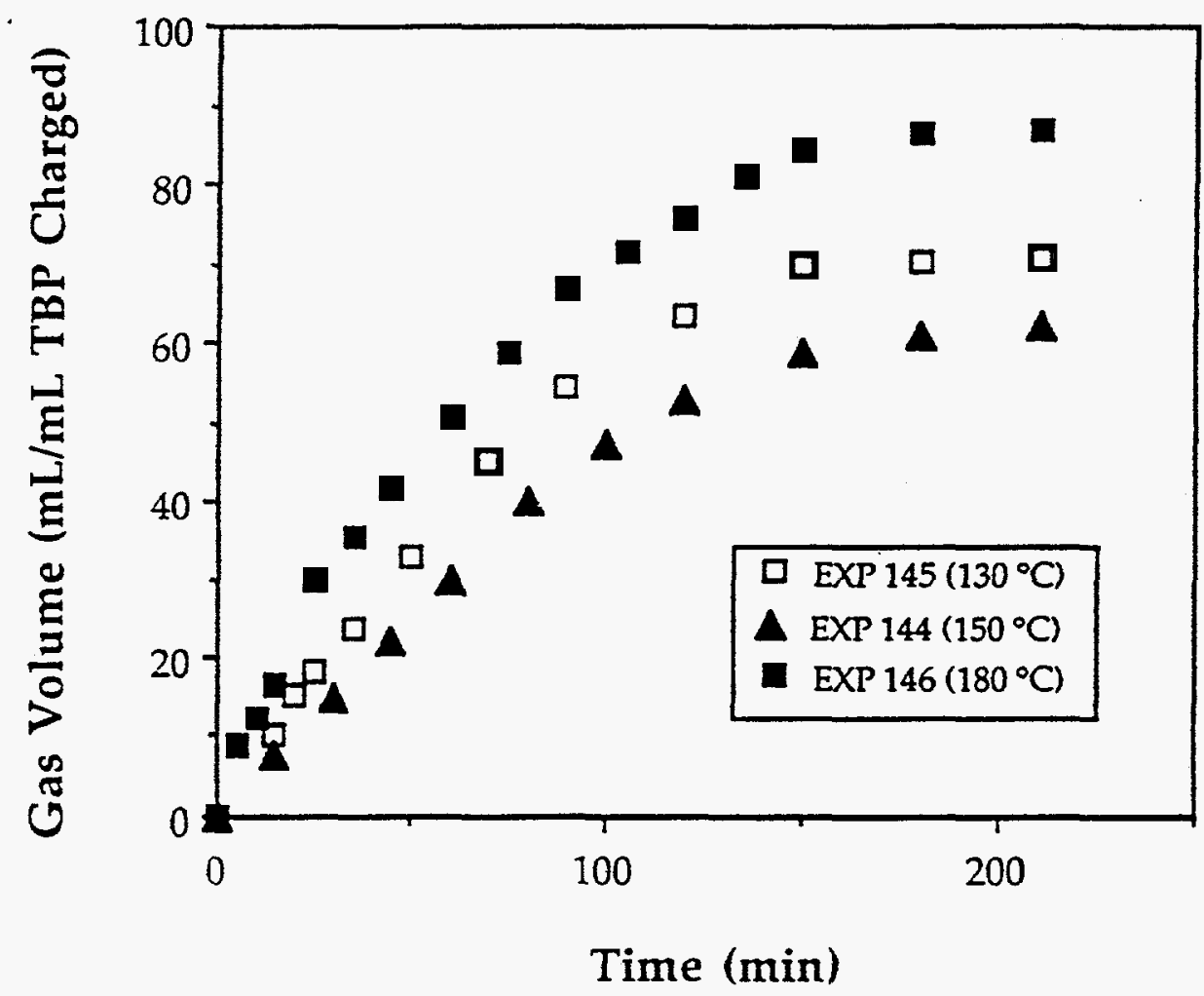


Fig. H-4 Effect of Temperature (SP,CP,6M,R)

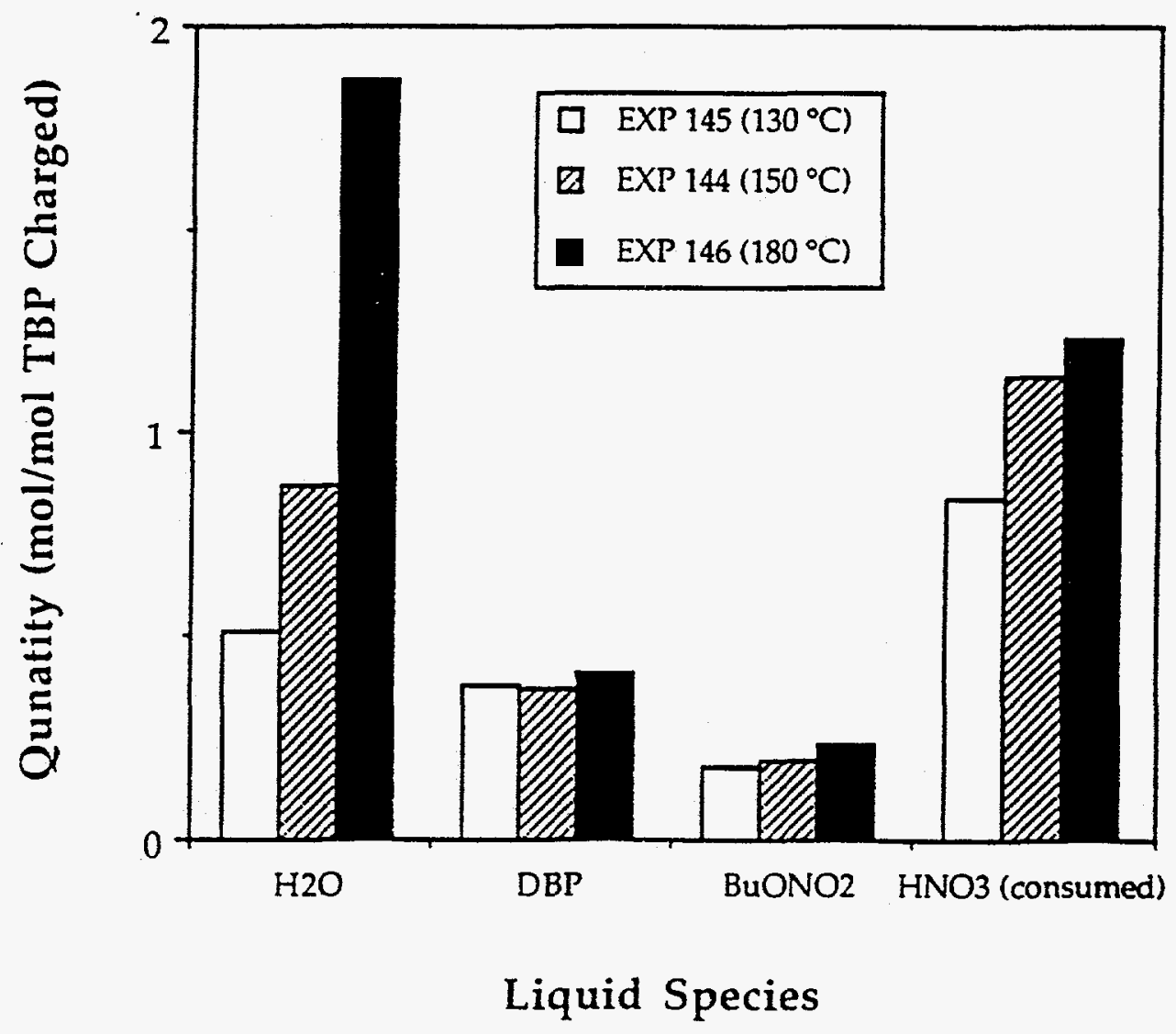


Fig. I-1 Effect of Temperature (SP,CV,6M)

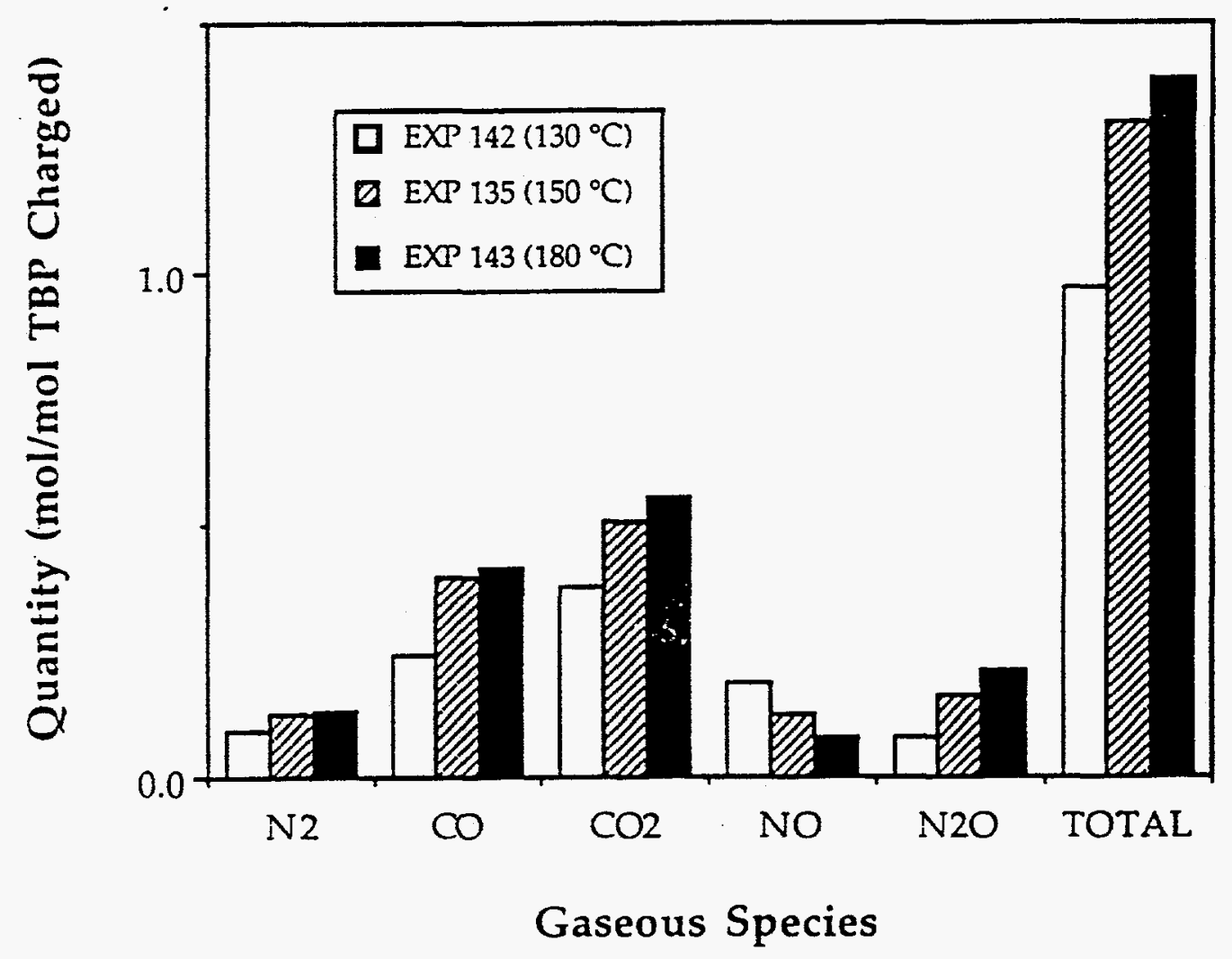


Fig. I-2 Effect of Temperature (SP,CV,6M)

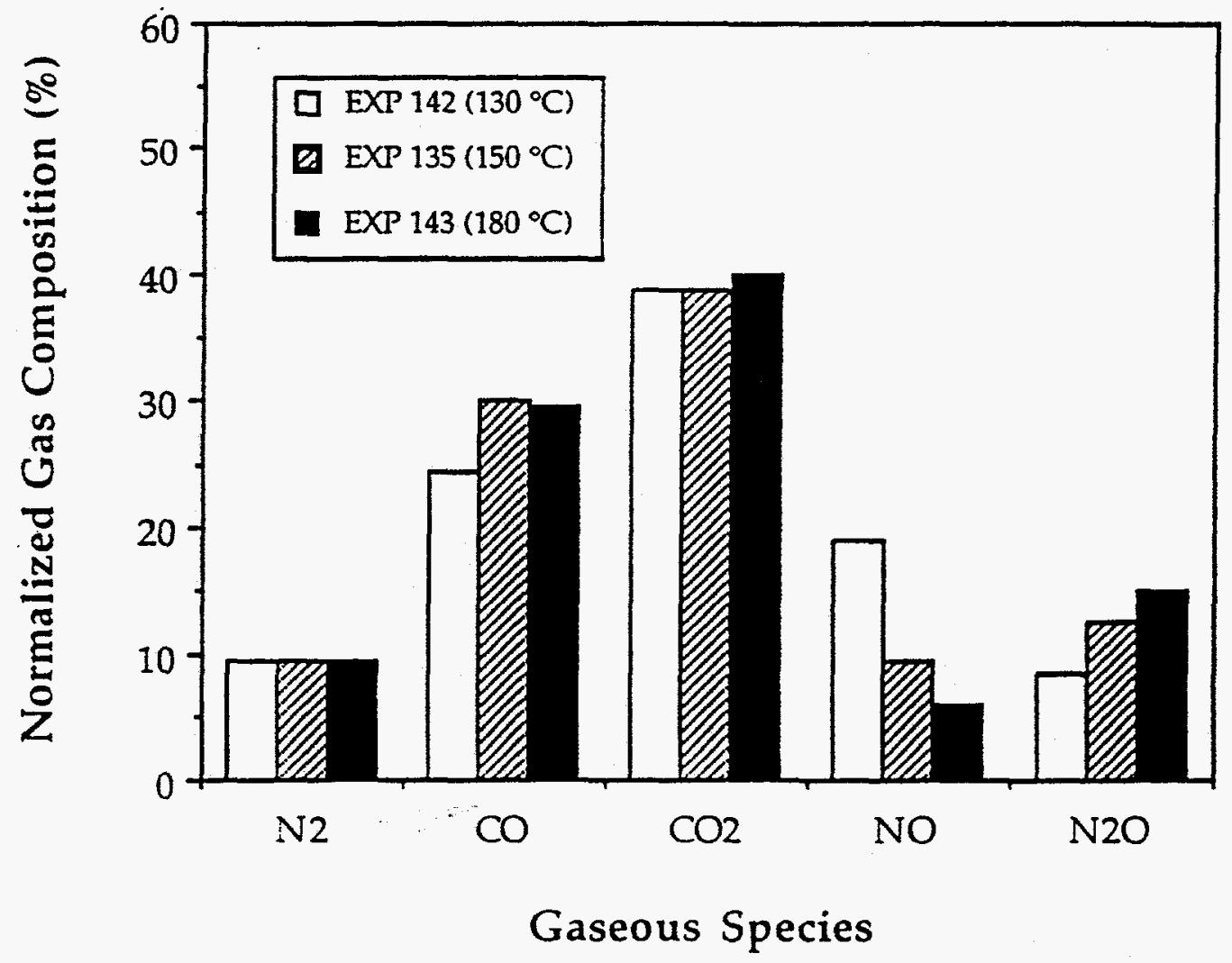


Fig. I-3 Effect of Temperature (SP,CV,6M)

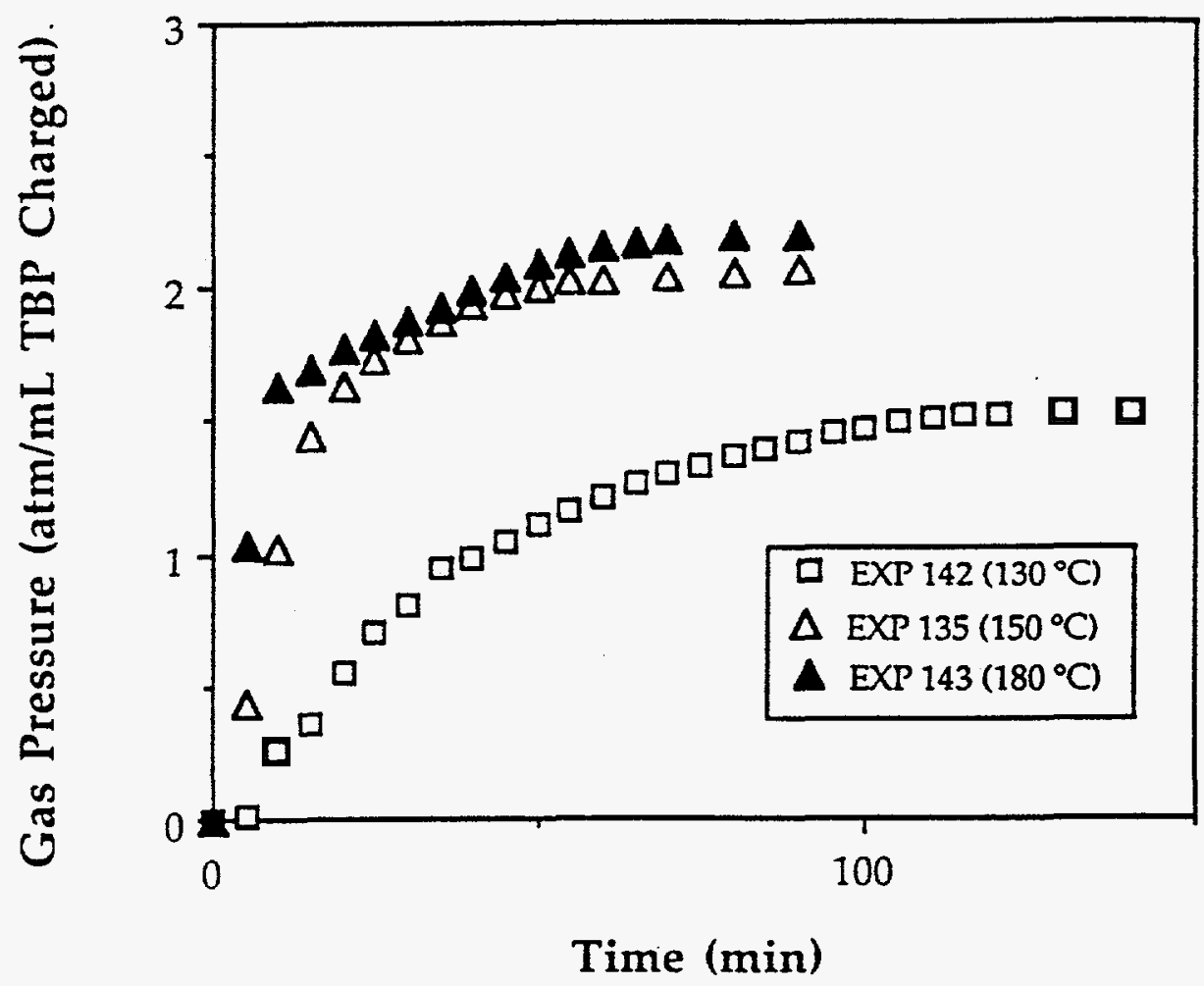


WSRC-RP-95-259

Fig. i-4 Effect of Temperature (SP,CV,6M)

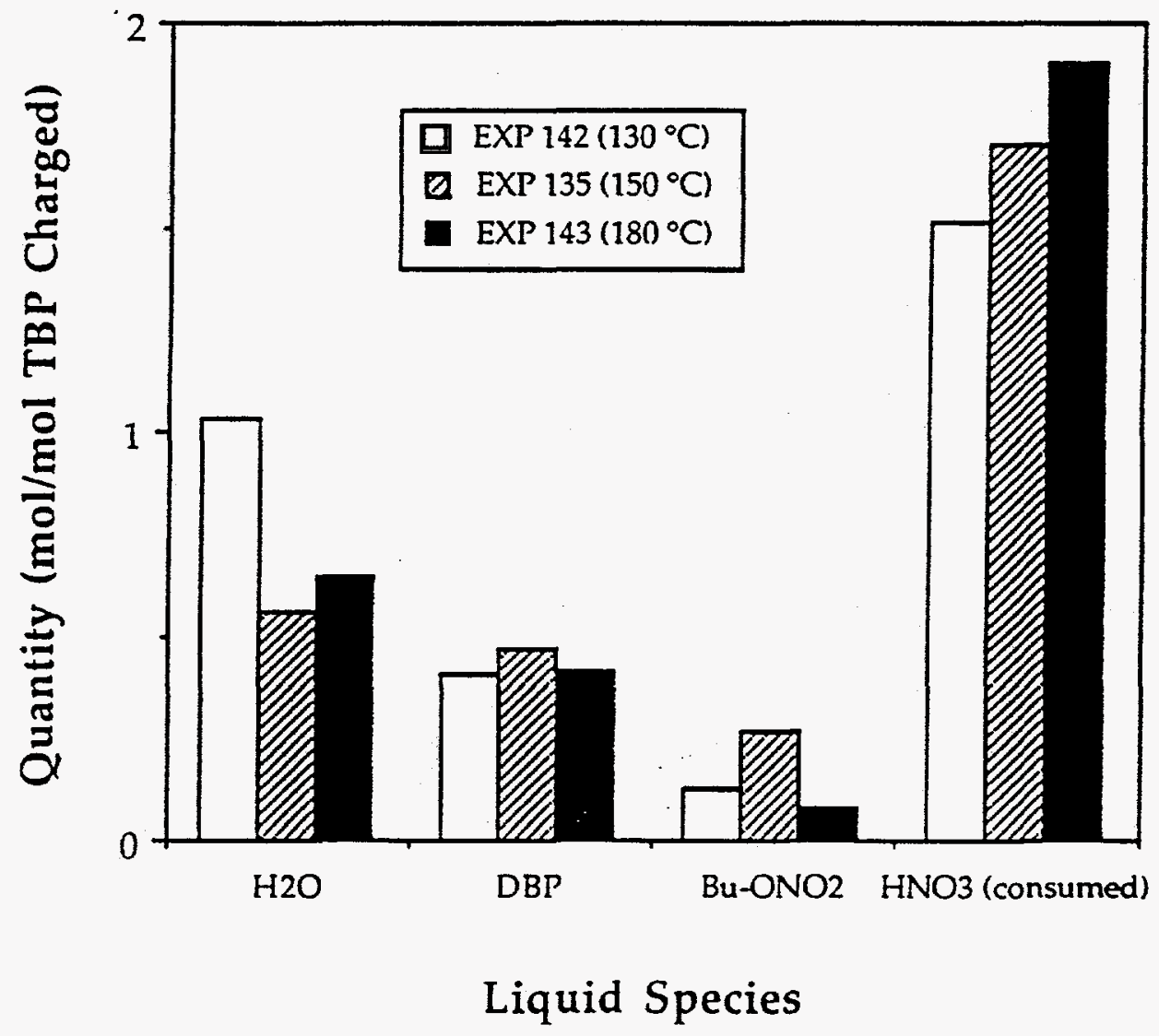


Fig. J-1 Effect of Temperature $(T P, C P, 6 M, D)$

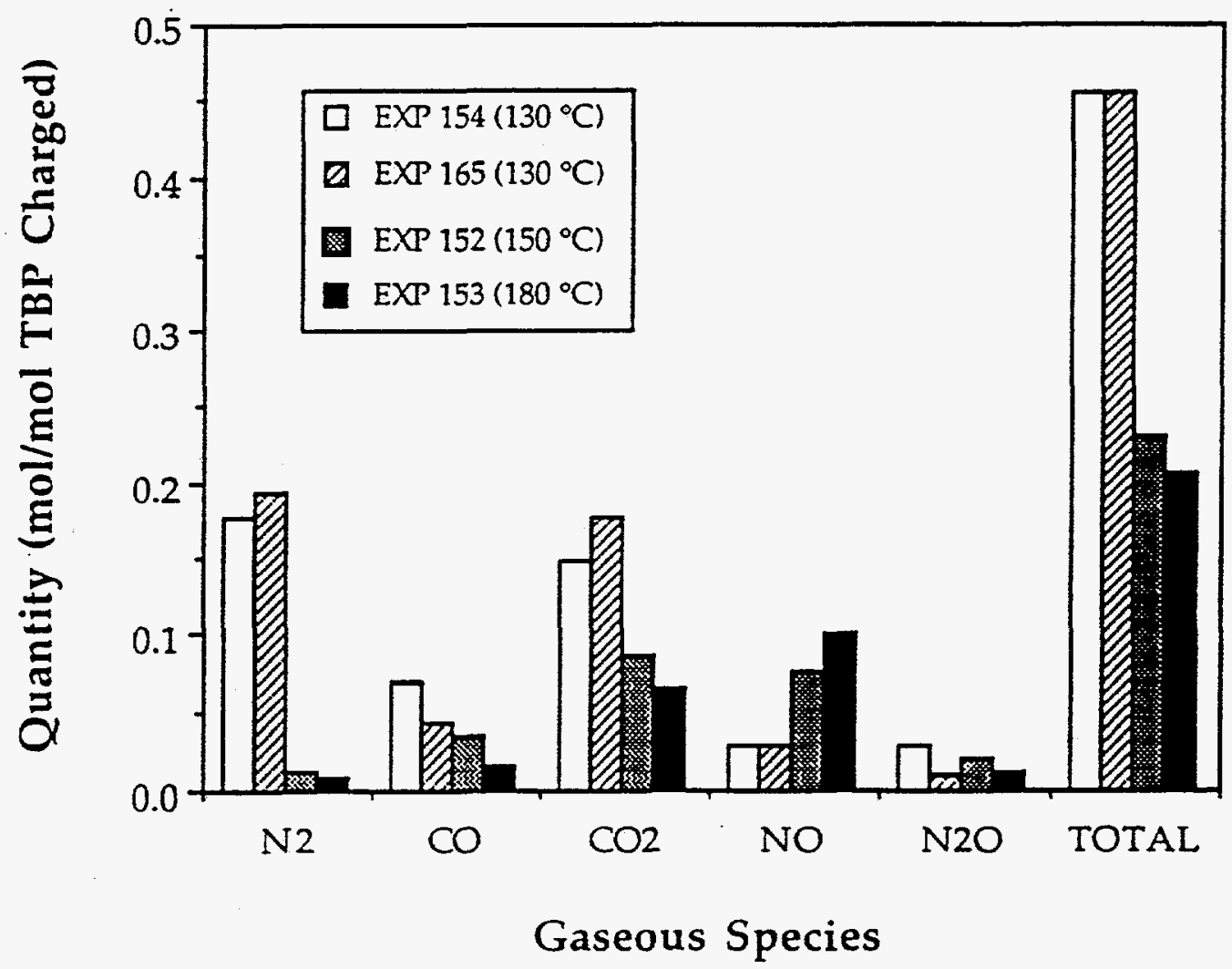


Fig. J-2 Effect of Temperature (TP,CP,6M,D)

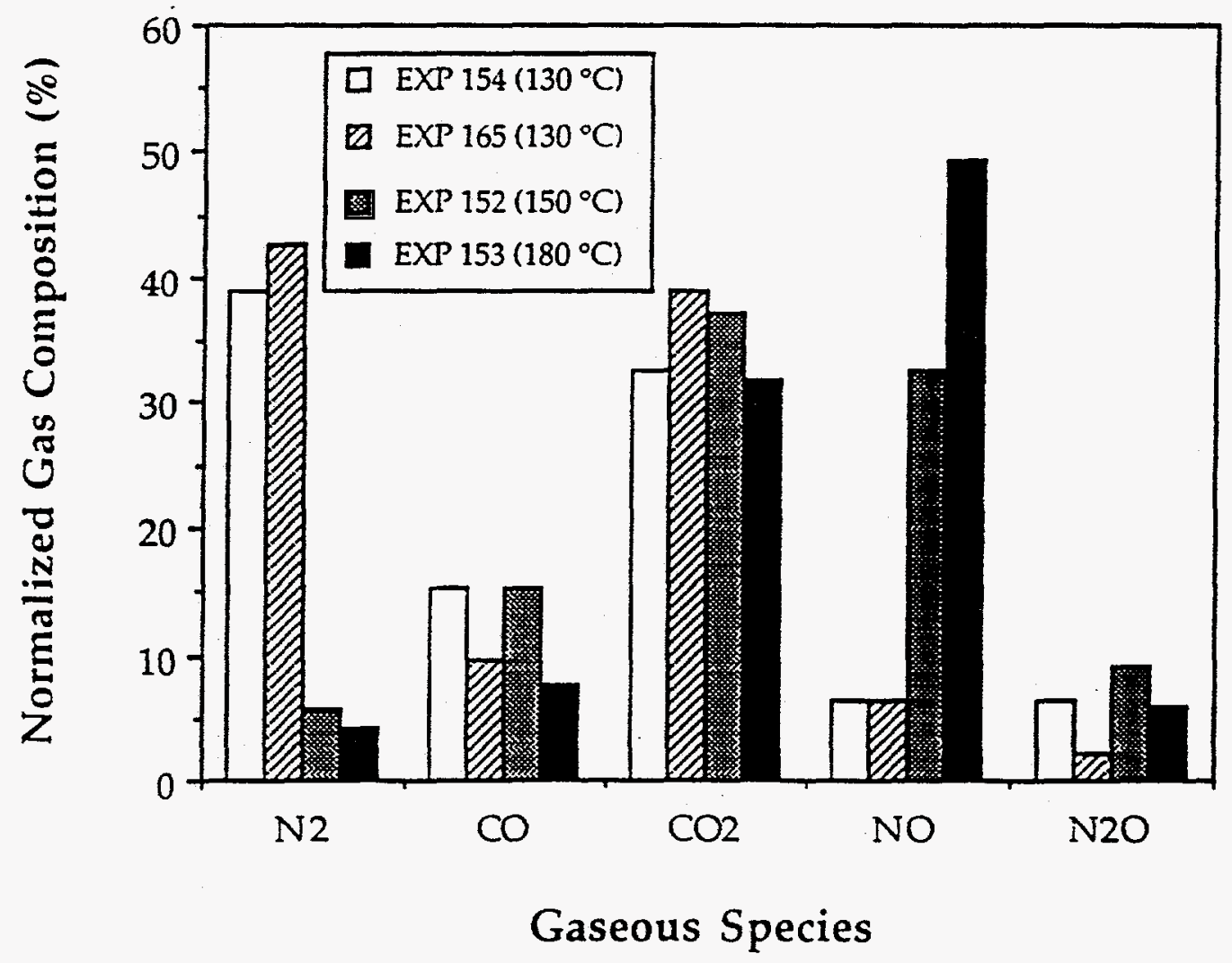


Fig. J-3 Effect of Temperature (TP,CP,6M,D)

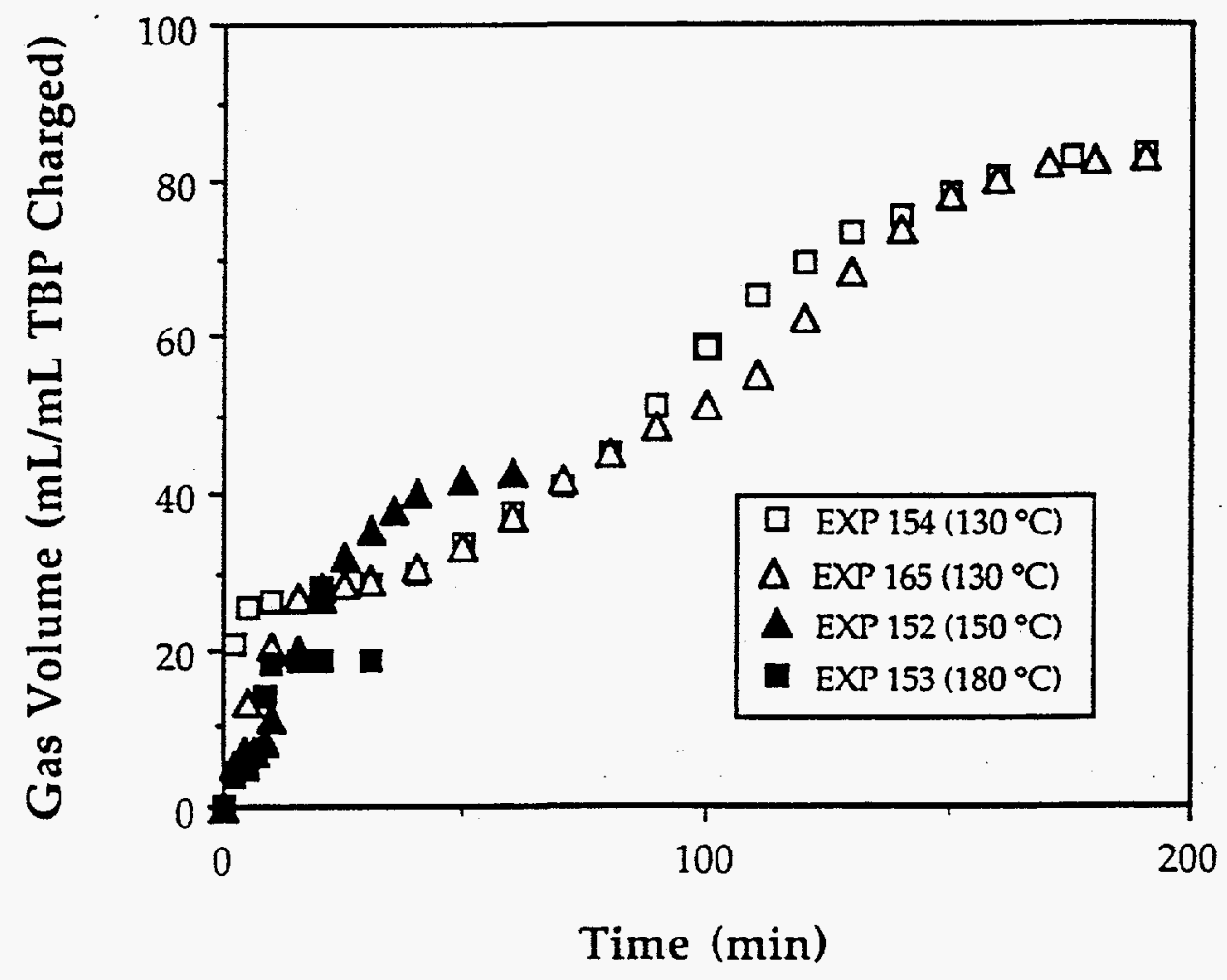


Fig. J-4 Effect of Temperature (TP, $\mathrm{CP}, 6 \mathrm{M}, \mathrm{D})$

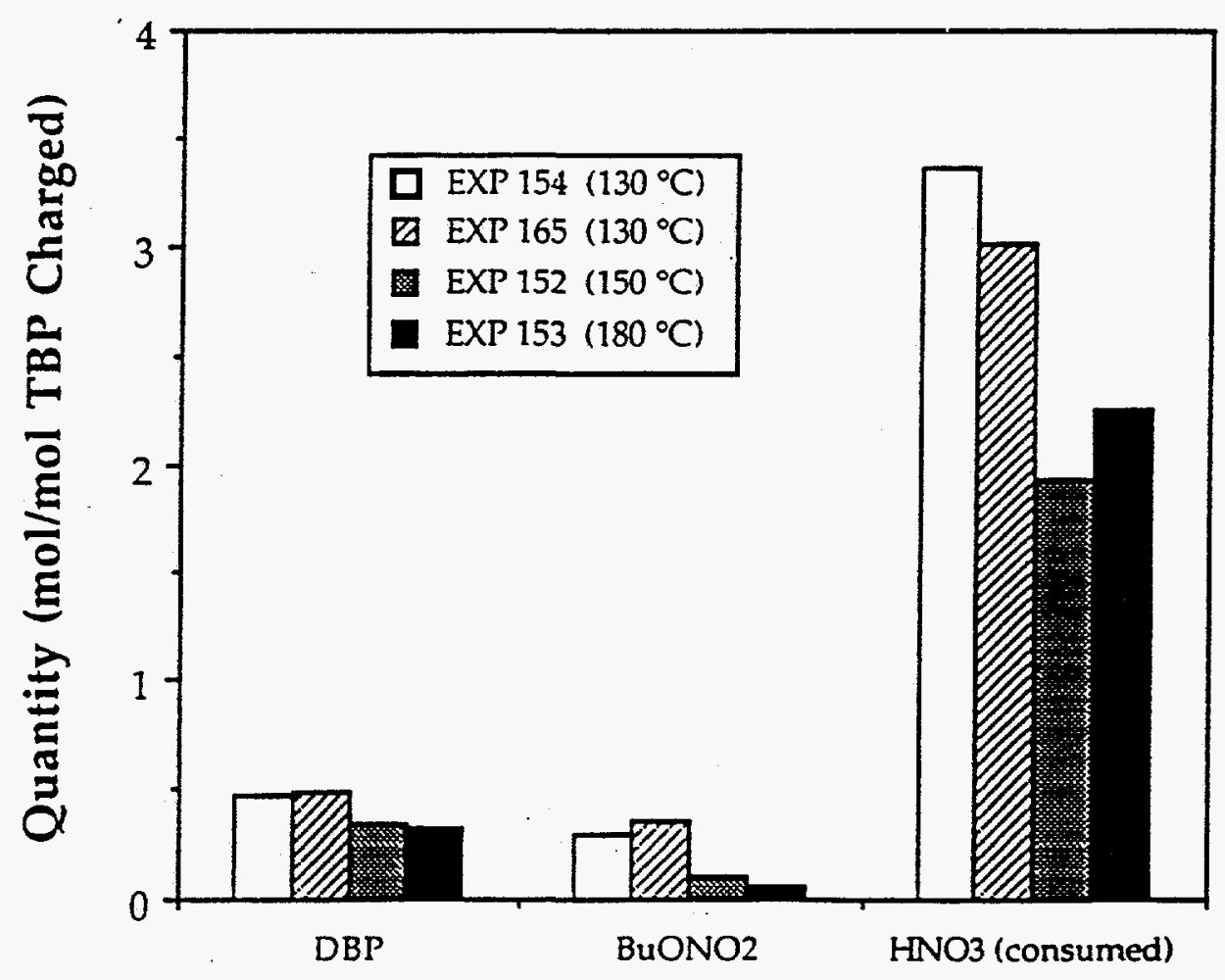

Liquid Species 
Fig. K-1 Effect of Temperature (TP, $\mathrm{CP}, 6 \mathrm{M}, \mathrm{R})$

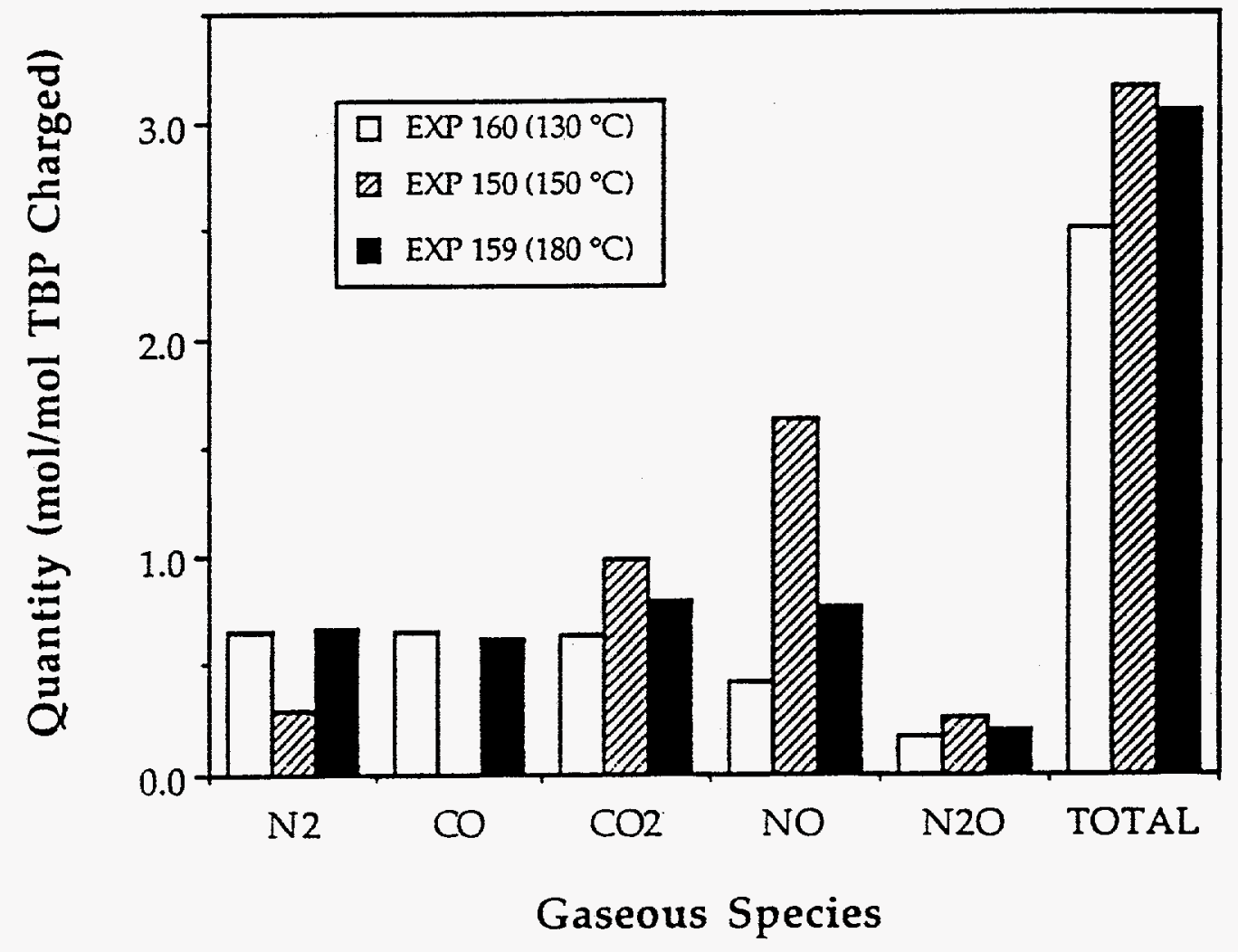


WSRC-RP-95-259

Fig. K-2 Effect of Temperature (TP, CP,6M,R)

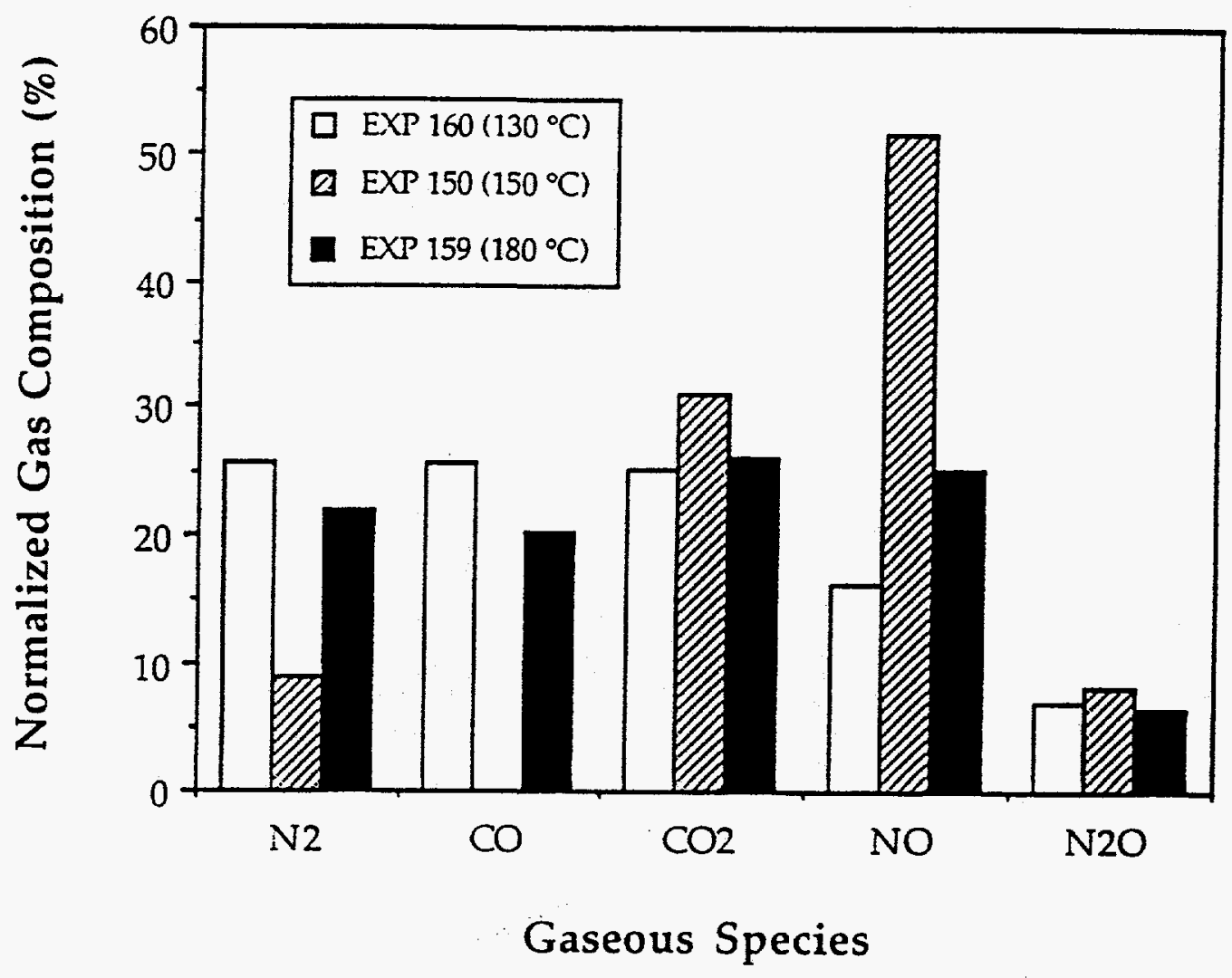


WSRC-RP-95-259

Fig. K-3 Effect of Temperature (TP,CP,6M, R)

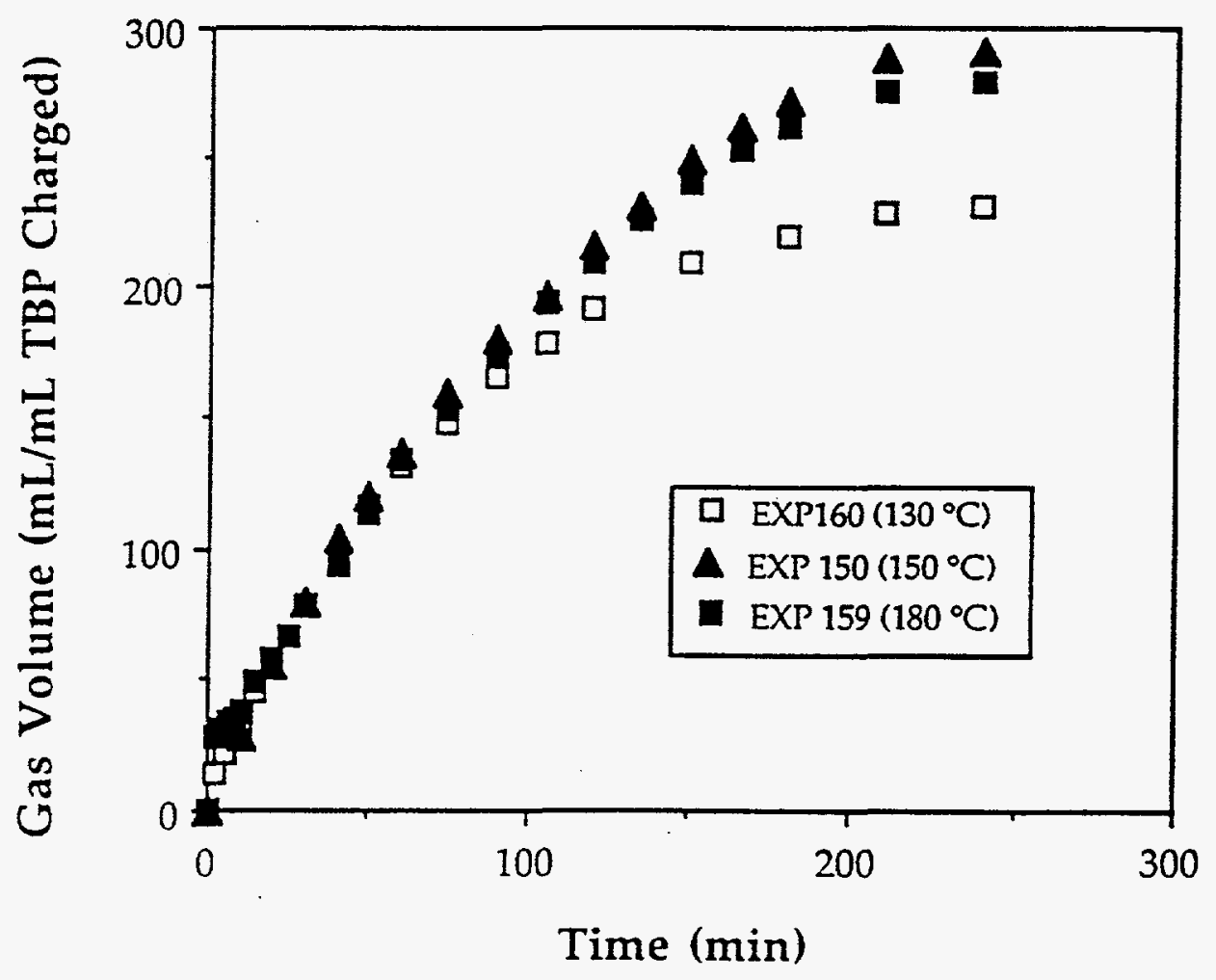

A-76 
Fig. $\mathrm{K}-4$ Effect of Temperature (TP,CP,6M,R)

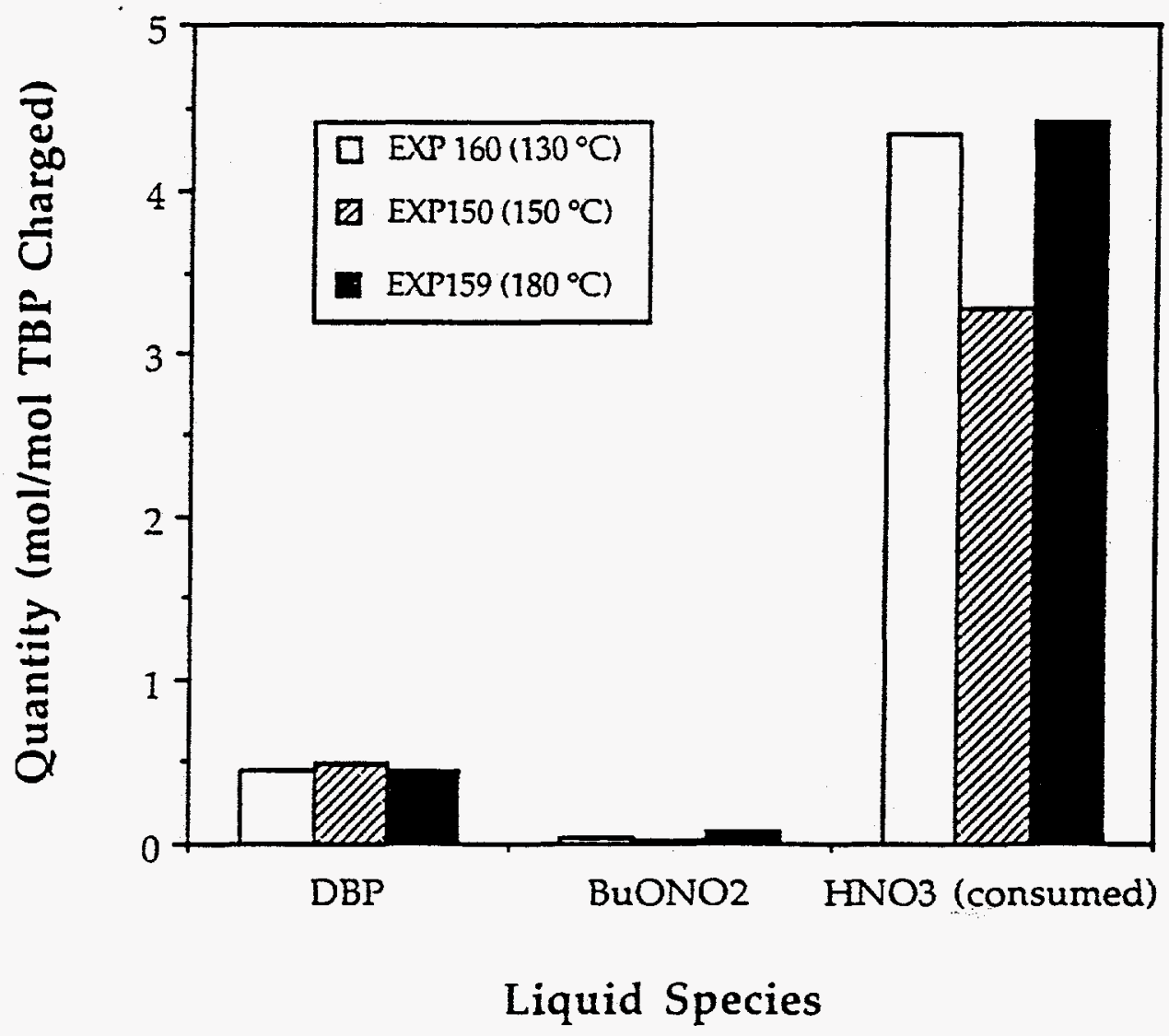


Fig. $\bar{L}-1$ Effect of Temperature (TP,CV,6M)

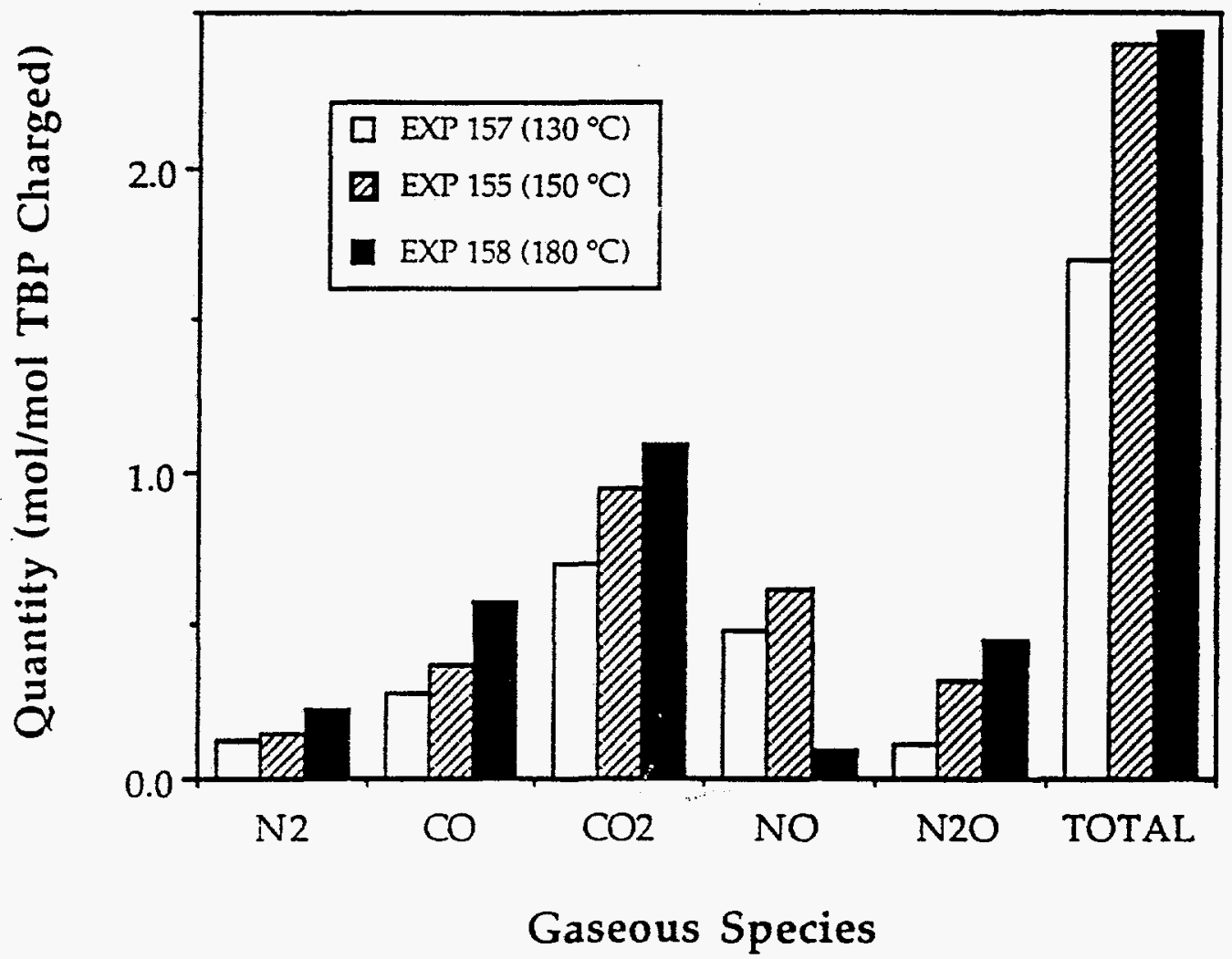


Fig. L-2 Effect of Temperature (TP,CV,6M)

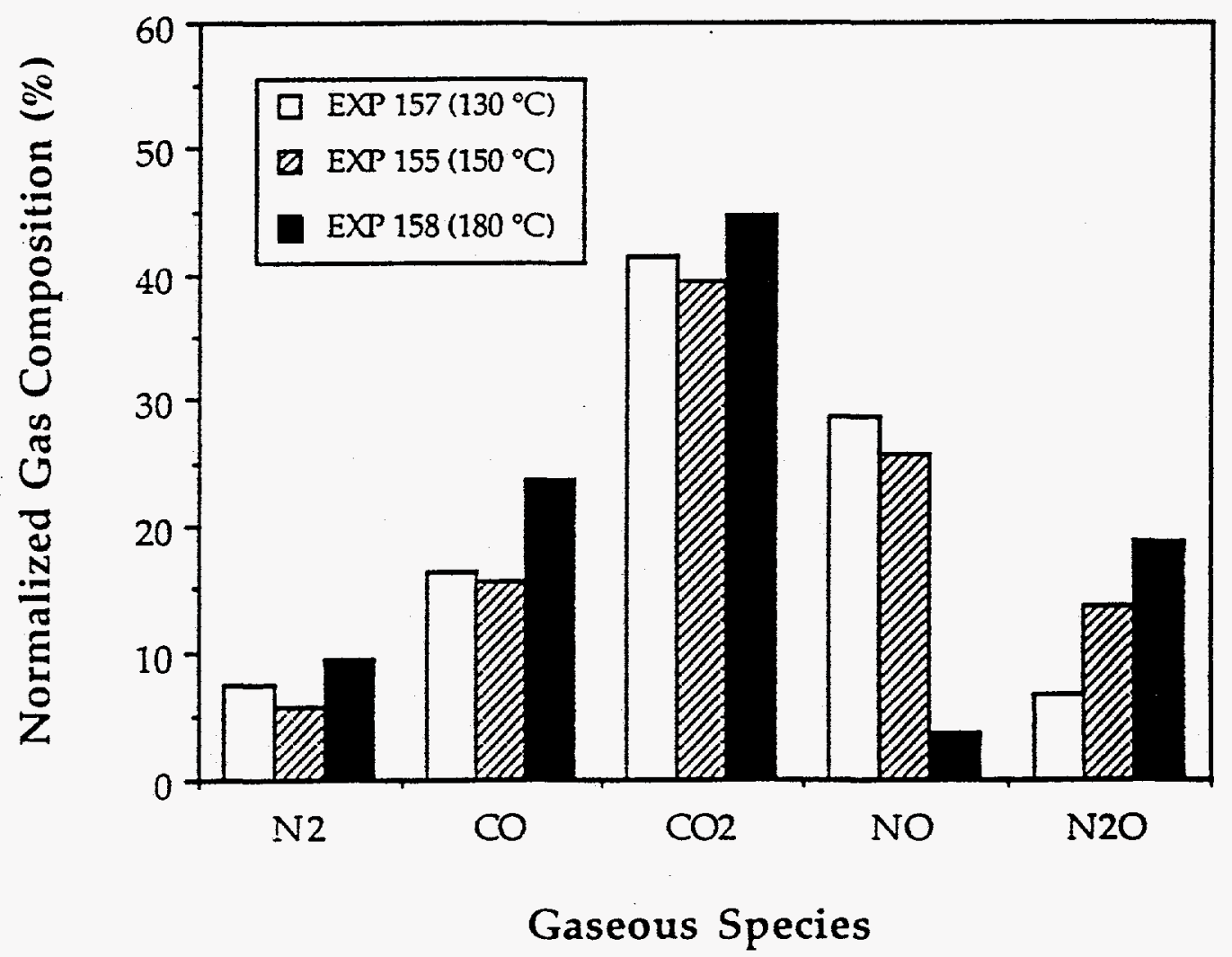


Fig. L-3 Effect of Temperature (TP,CV,6M)

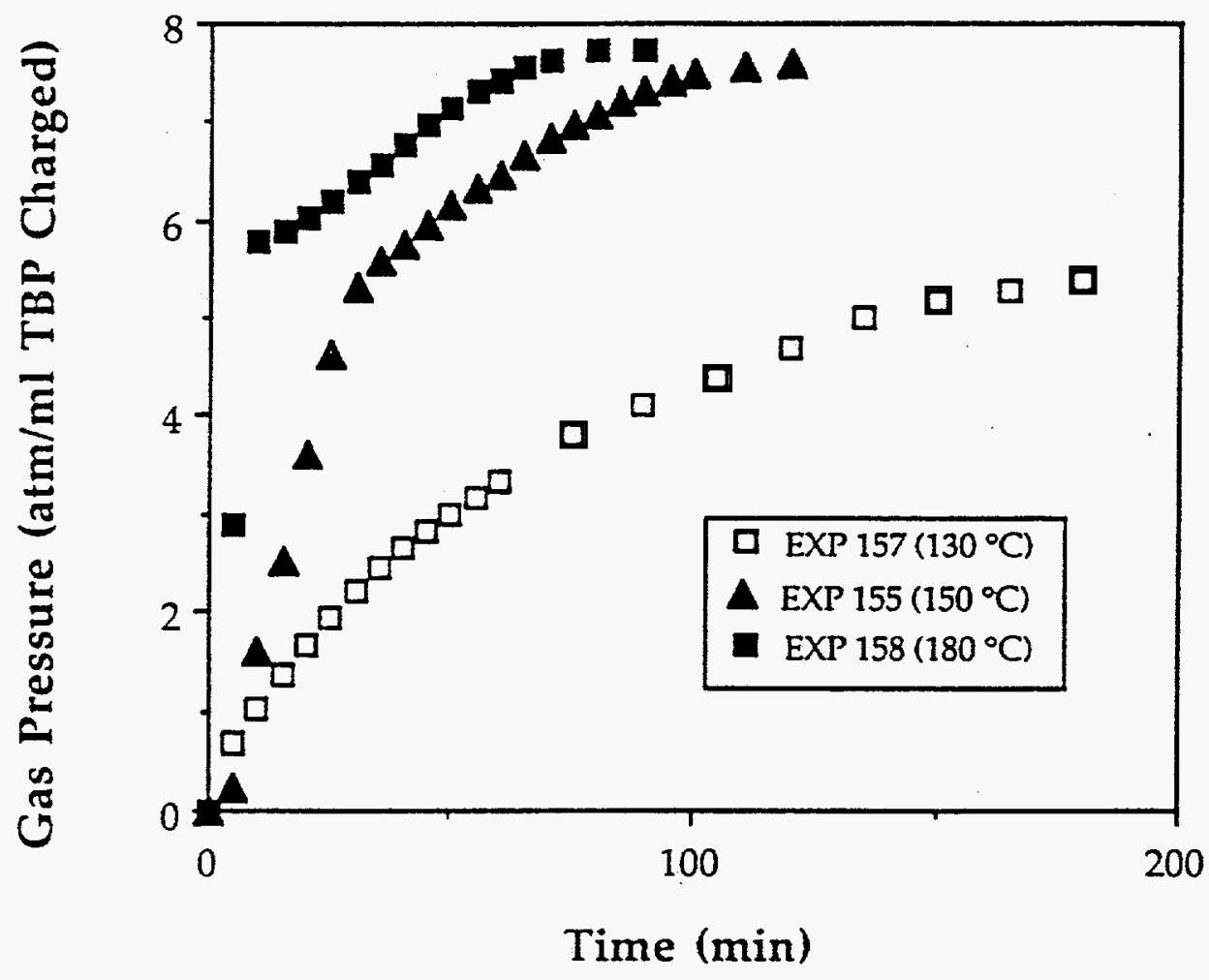


Fig. L-4 Effect of Temperature (TP,CV,6M)

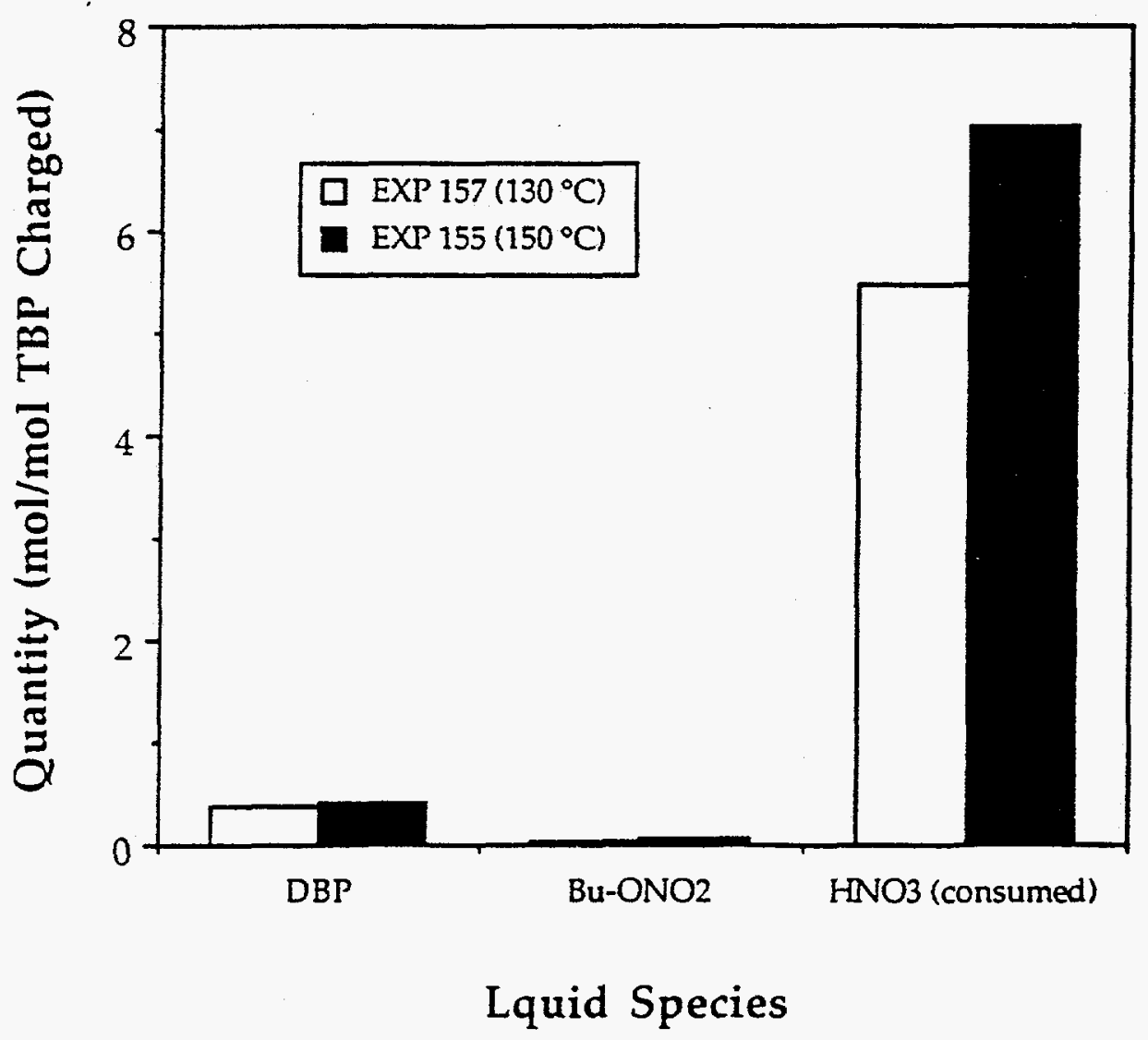




\section{APPENDICES M-N}

\section{EFFECT OF $\mathrm{Zr}^{4+}$}

Appendices $\mathrm{M}$ and $\mathrm{N}$ provide graphical summaries of the effects of presence of $\mathrm{Zr}^{4+}$ on single phase reactions of TBP in 6M nitric acid with either constant pressure (Appendix M) or constant volume (Appendix $N$ ) conditions. 
Fig. M-1 Effect of $\mathrm{Zr} 4+\left(\mathrm{SP}, \mathrm{CP}, 6 \mathrm{M}, \mathrm{D}, 150^{\circ} \mathrm{C}\right)$

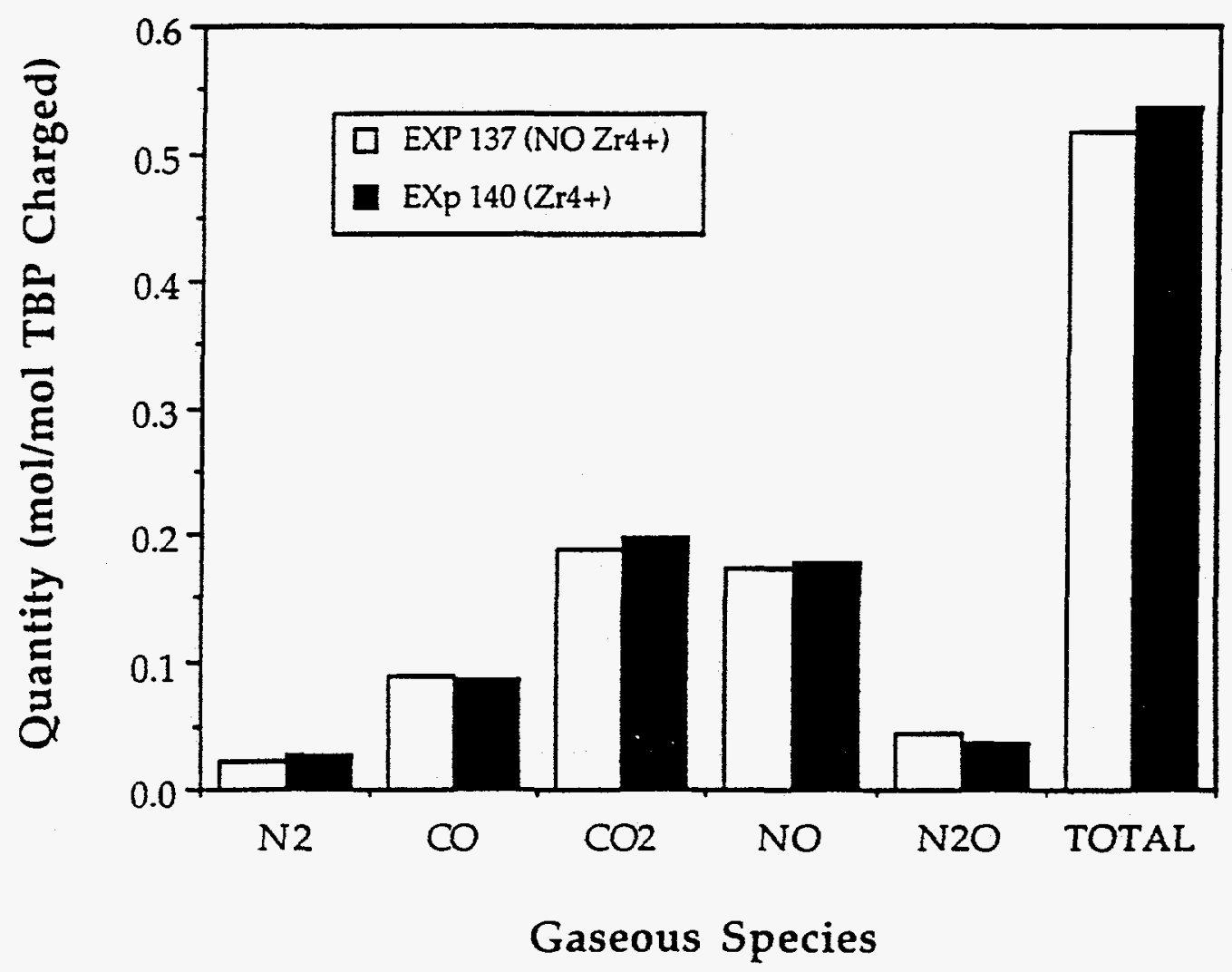


Fig. M-2 Effect of $\mathrm{Zr} 4+\left(\mathrm{SP}, \mathrm{CP}, 6 \mathrm{M}, \mathrm{D}, 150^{\circ} \mathrm{C}\right)$

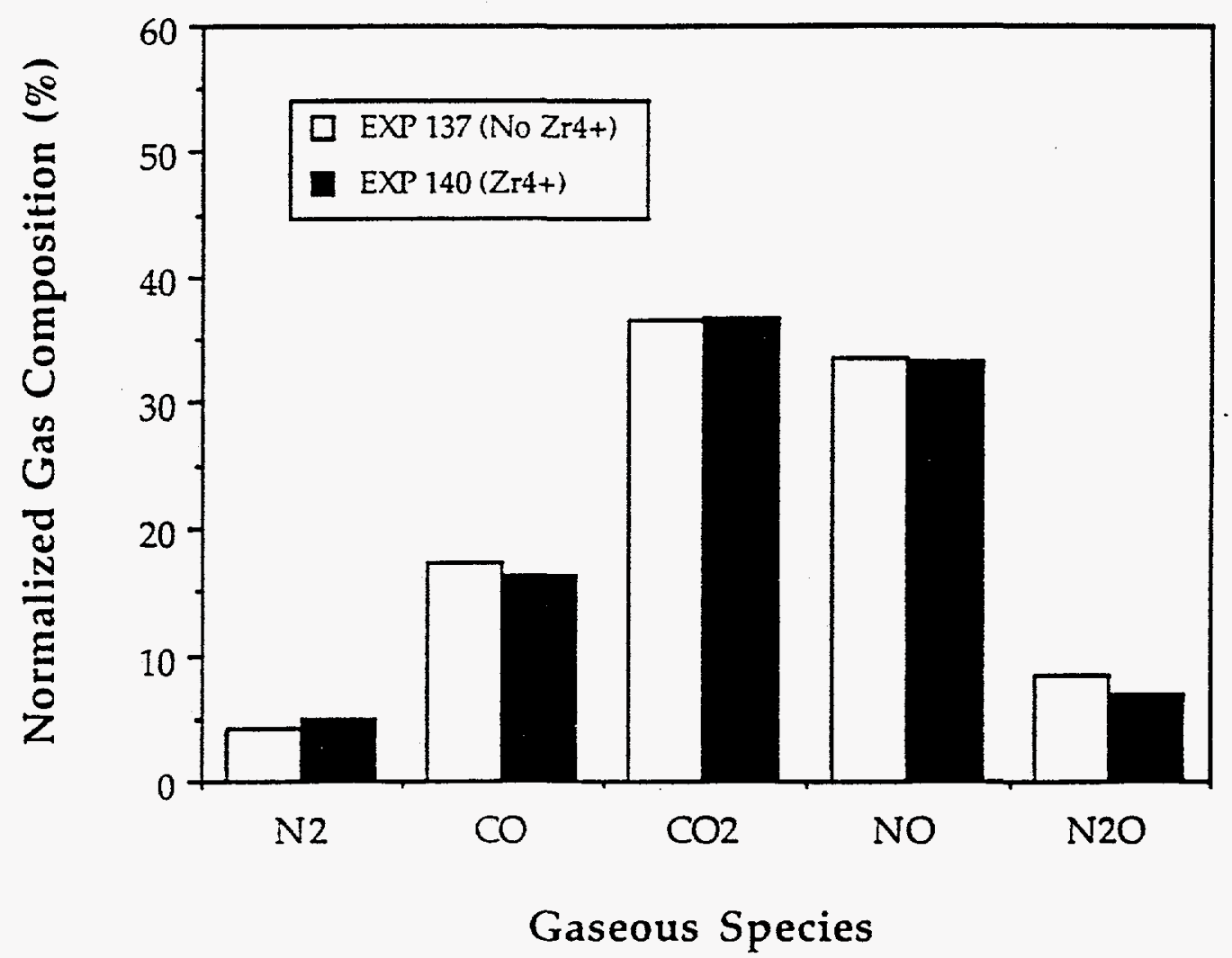


WSRC-RP-95-259

Fig. M-3 Effect of $\mathrm{Zr} 4+\left(\mathrm{SP}, \mathrm{CP}, 6 \mathrm{M}, \mathrm{D}, 150^{\circ} \mathrm{C}\right)$

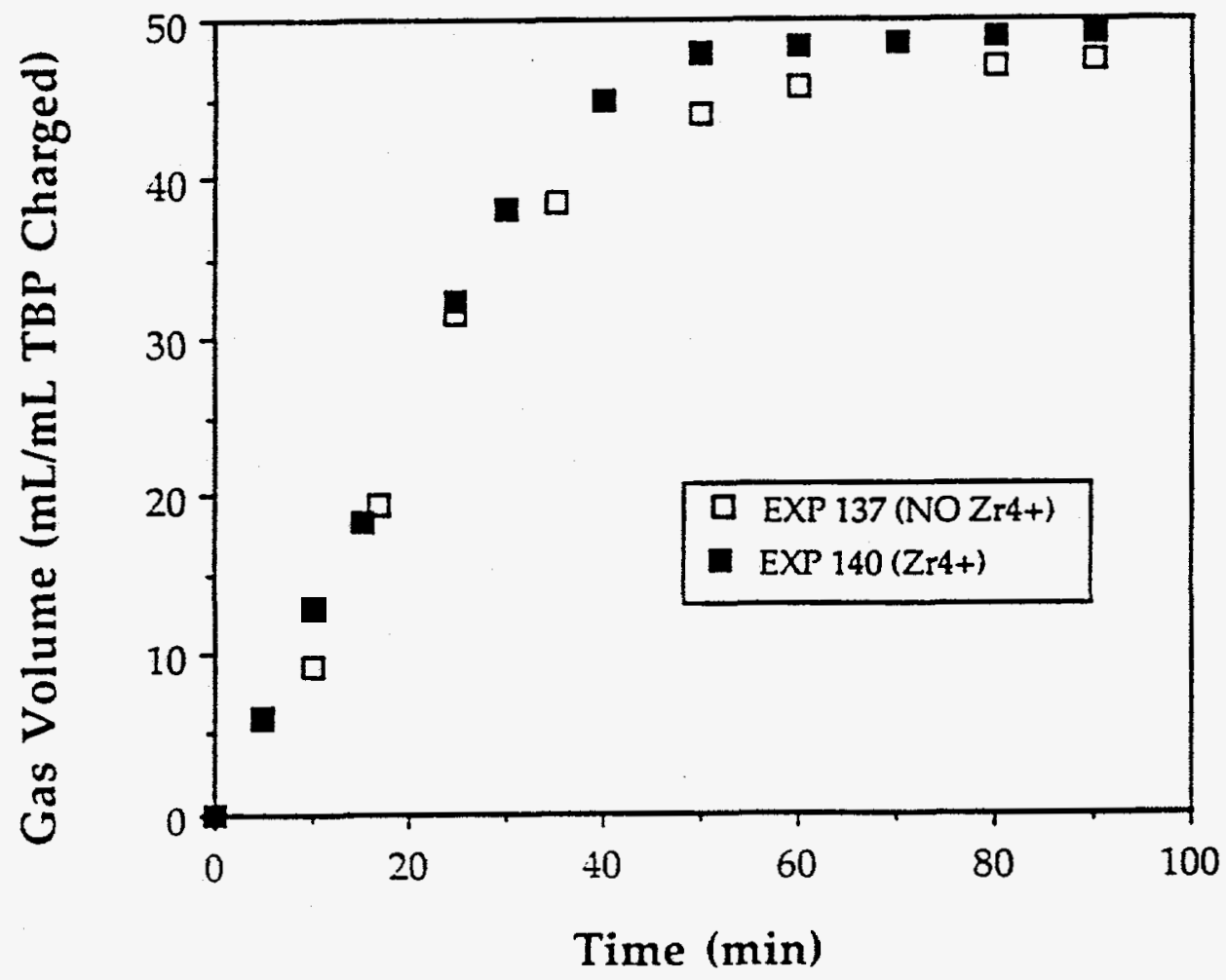


Fig. M-4 Effect of $\mathrm{Zr} 4+\left(\mathrm{SP}, \mathrm{CP}, 6 \mathrm{M}, \mathrm{D}, 150^{\circ} \mathrm{C}\right)$

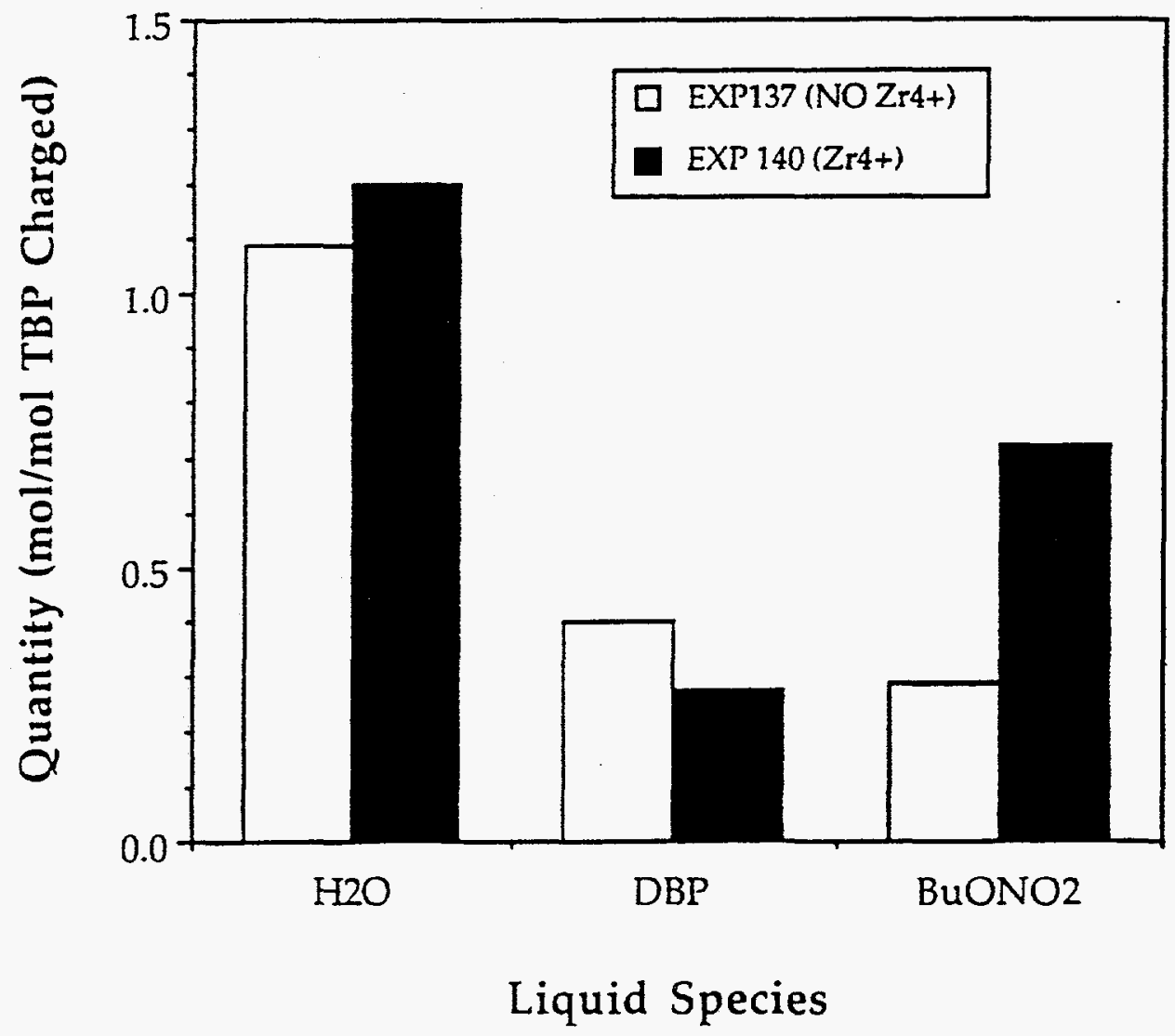


Fig. N-1 Effect of $\mathrm{Zr} 4+\left(\mathrm{SP}, \mathrm{CV}, 6 \mathrm{M}, 150^{\circ} \mathrm{C}\right)$

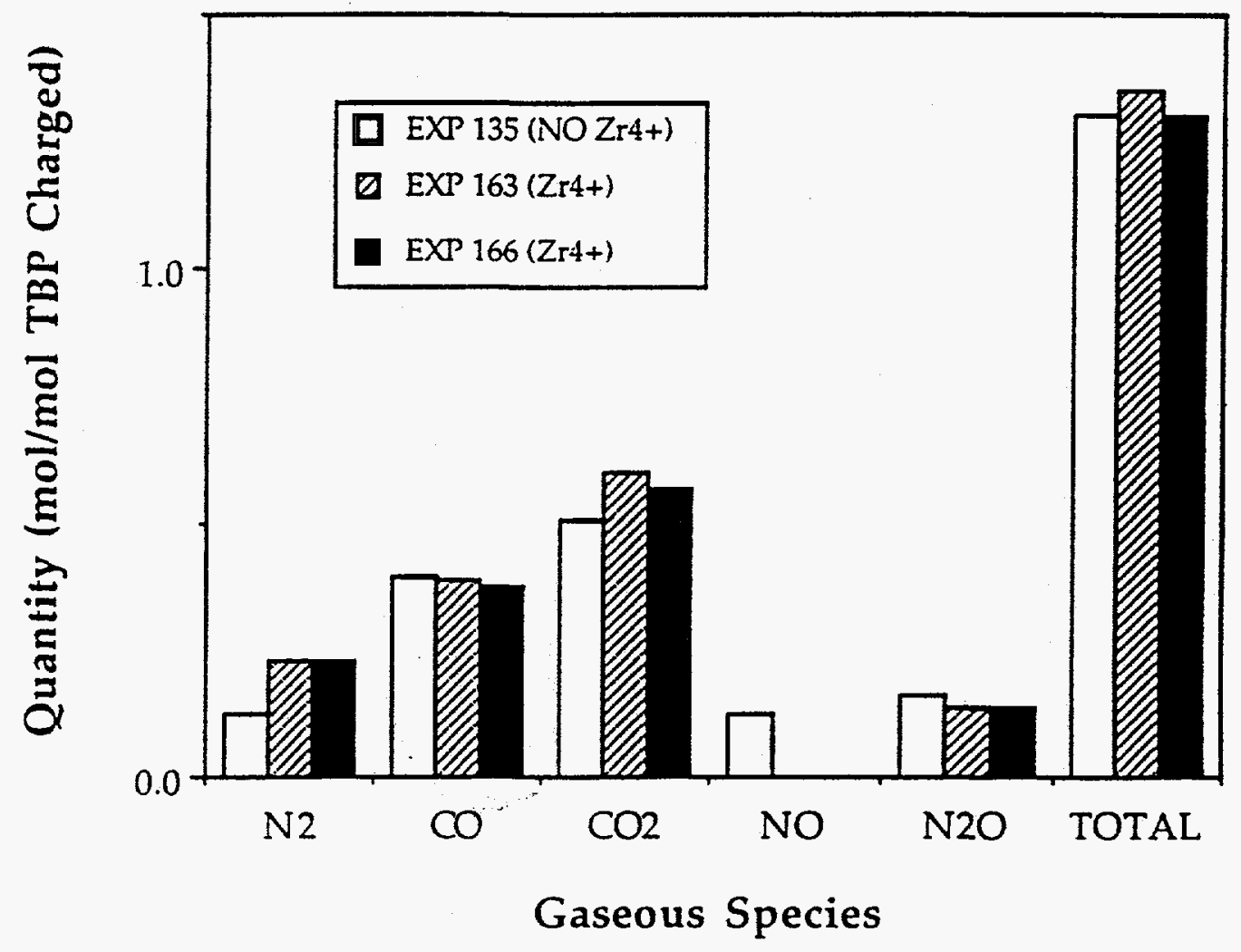


WSRC-RP-95-259

Fig. N-2 Effect of $\mathrm{Zr} 4+\left(\mathrm{SP}, \mathrm{CV}, 6 \mathrm{M}, 150^{\circ} \mathrm{C}\right)$

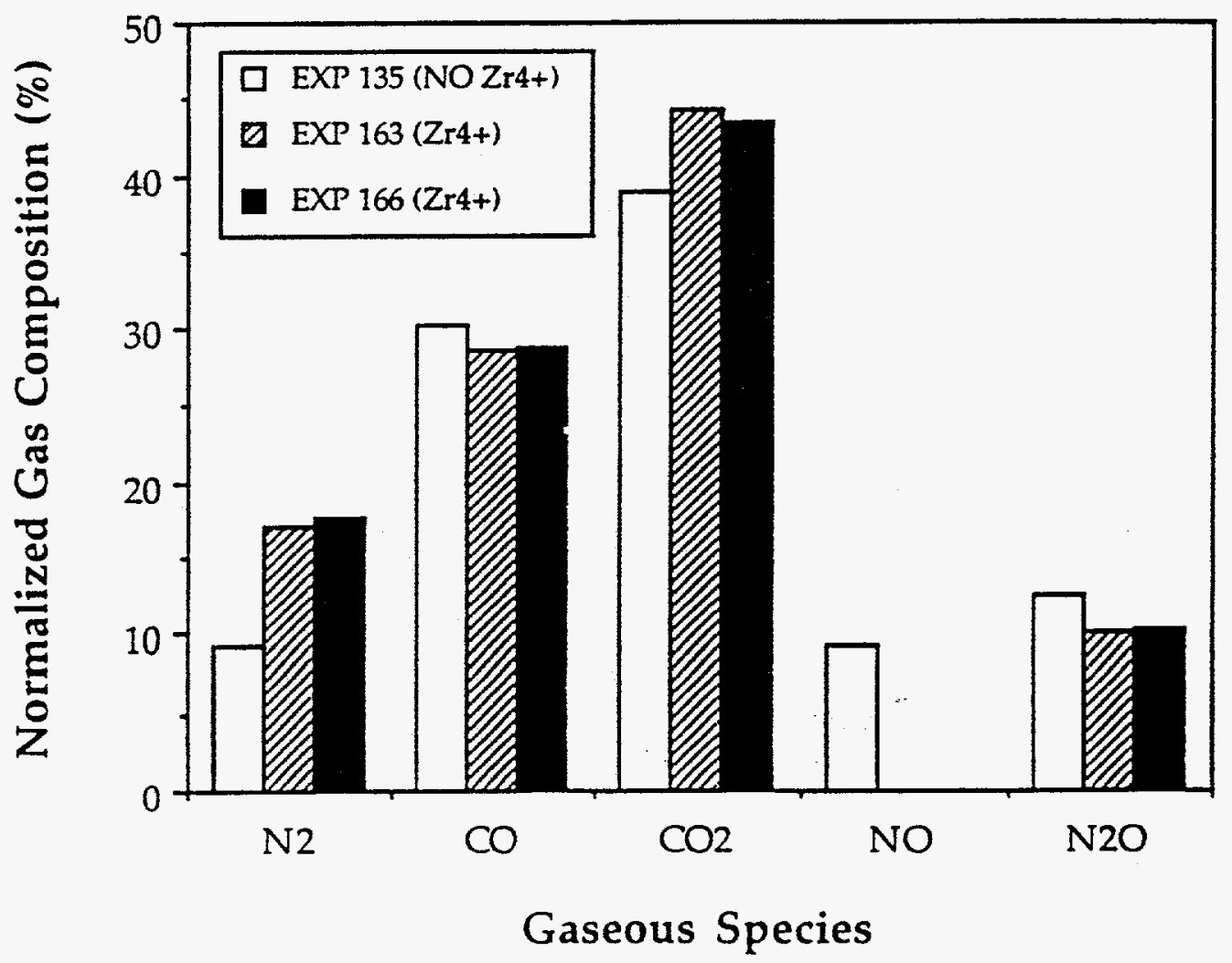


Fig. N-3 Effect of $\mathrm{Zr} 4+\left(\mathrm{SP}, \mathrm{CV}, 6 \mathrm{M}, 150^{\circ} \mathrm{C}\right)$

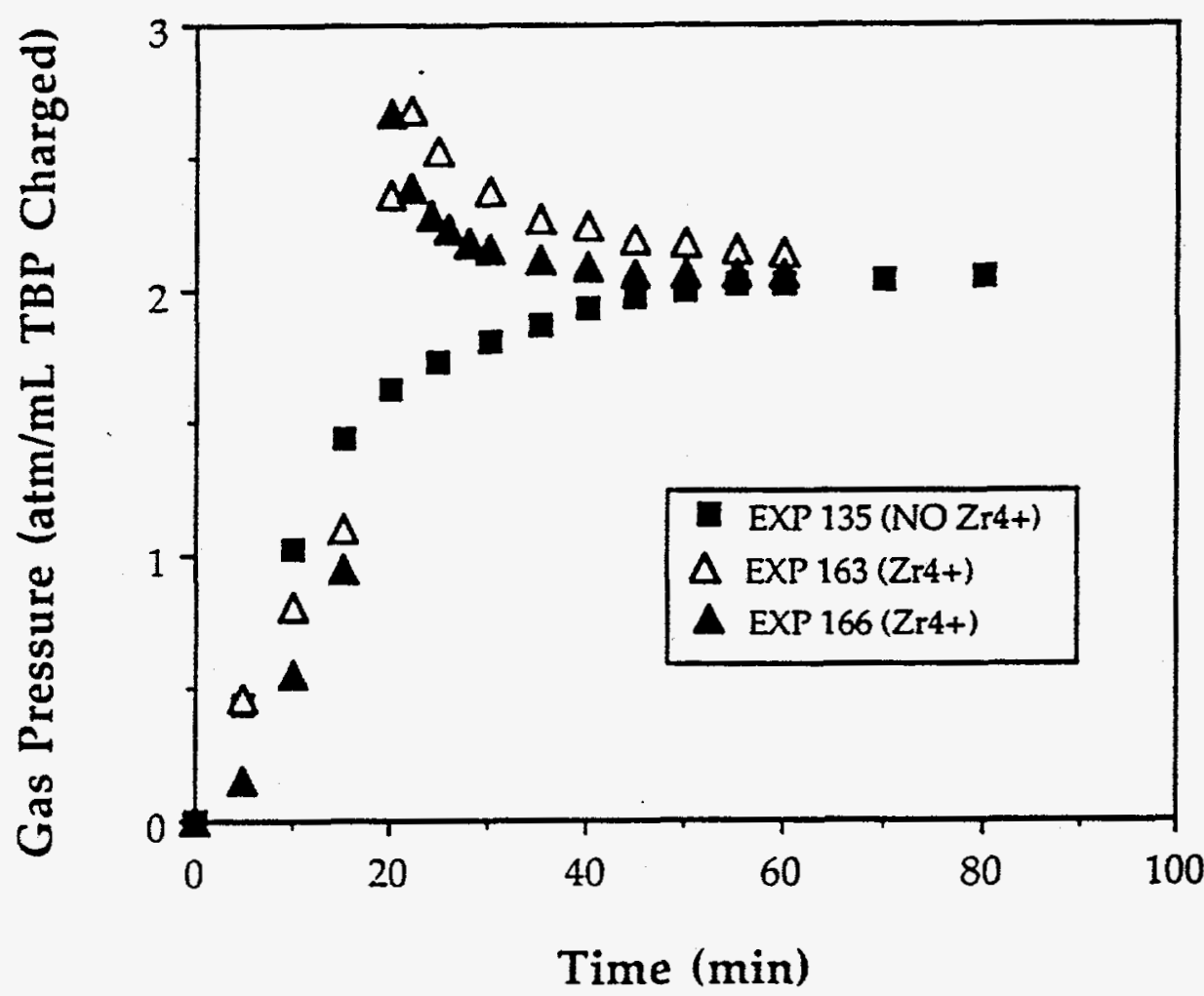


WSRC-RP-95-259

\section{APPENDIX 0}

\section{COMPA RISOIY BETWEEN DISTILLATION AND REFLUX CONDITIONS}

This Appendix provides graphical summaries of the effect of experimental conditions (distillation versus reflux) for reaction of TBP under single phase, constant pressure, $6 \mathrm{M}$, and $150^{\circ} \mathrm{C}$ conditions. 


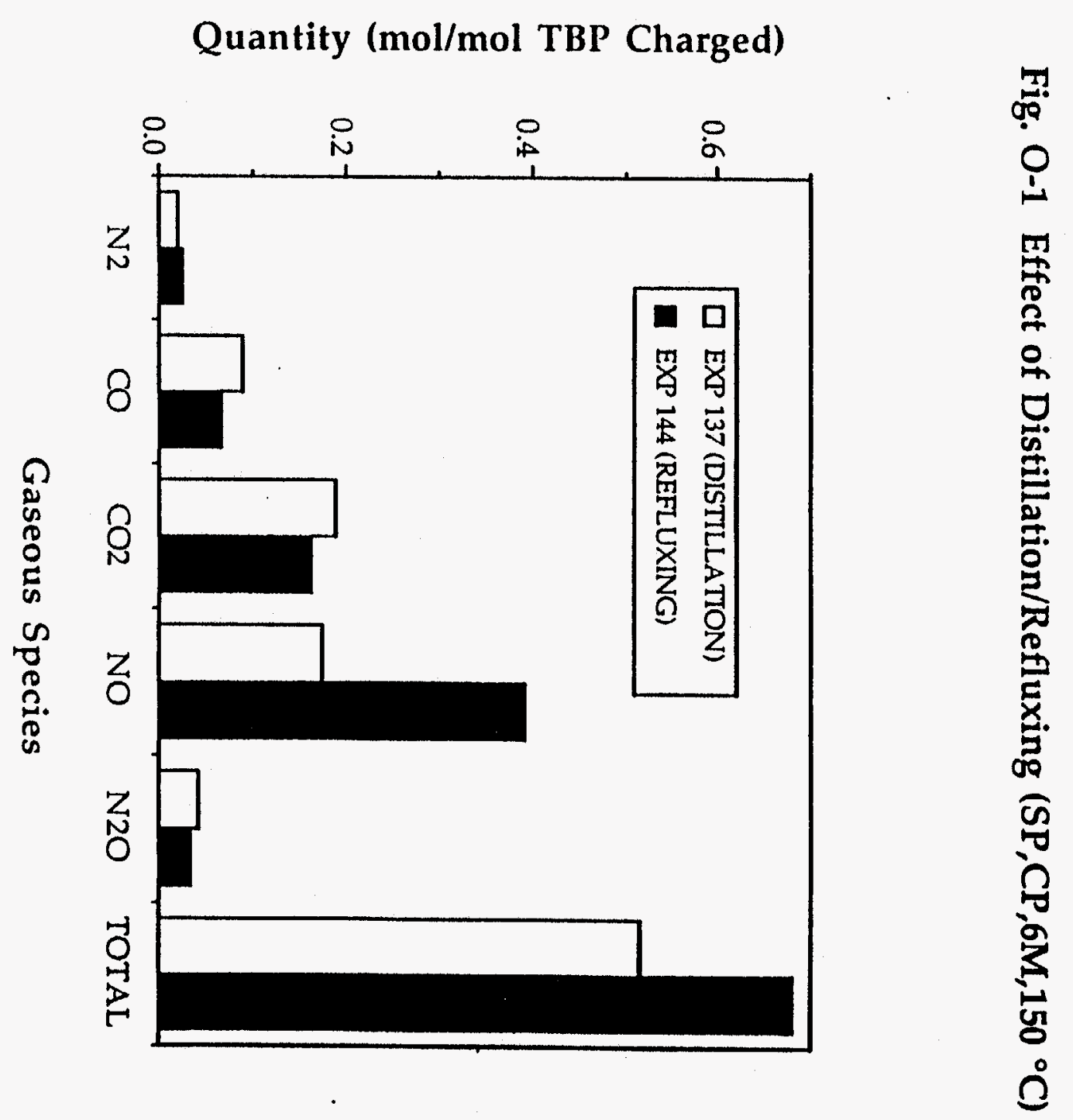


Fig. O-2 Effect of Distillation/Refluxing (SP,CP,6M,150 $\left.{ }^{\circ} \mathrm{C}\right)$

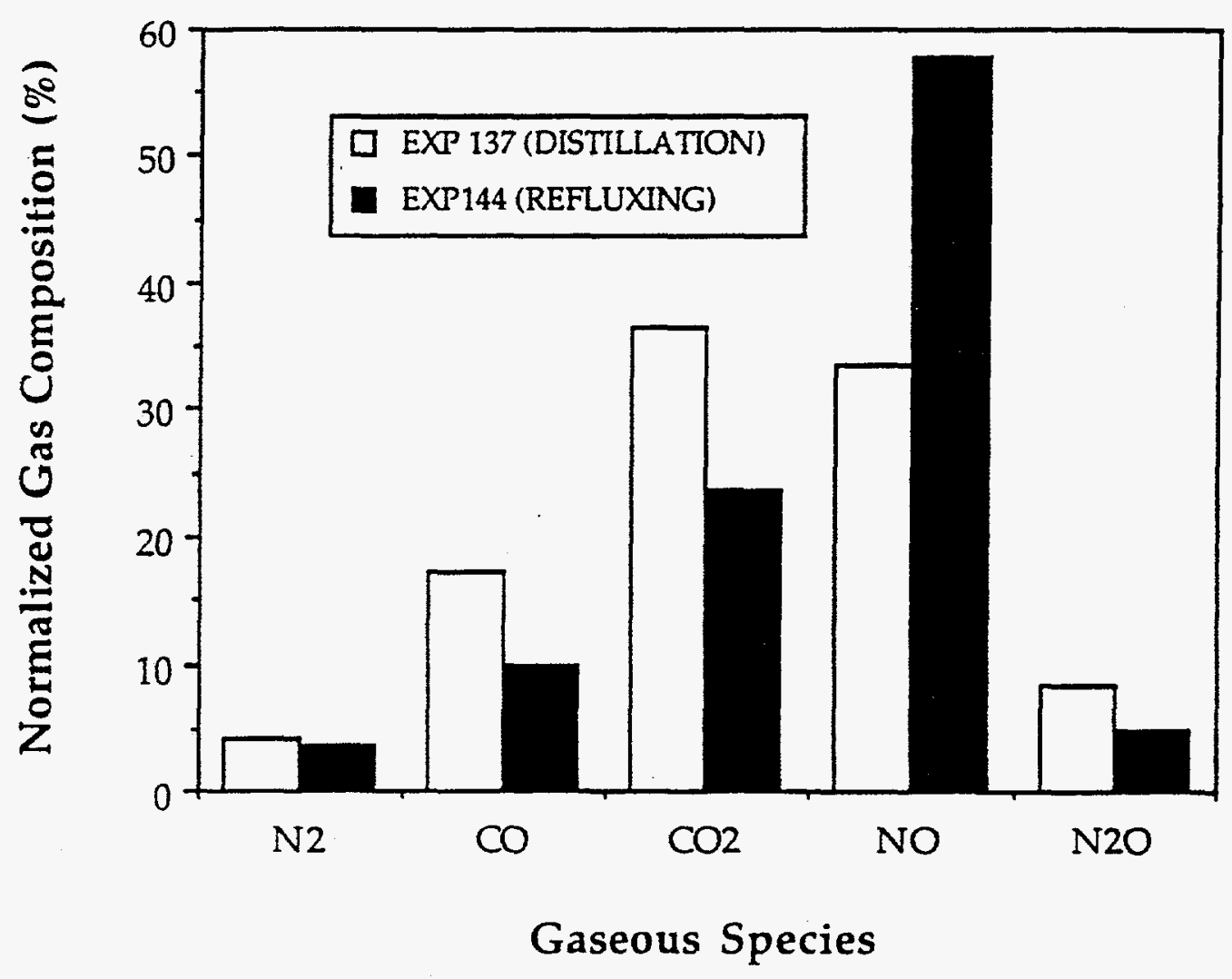


Fig. O-3 Effect of Distillation/Refluxing (SP, $\left.\mathrm{CP}, 6 \mathrm{M}, 150^{\circ} \mathrm{C}\right)$

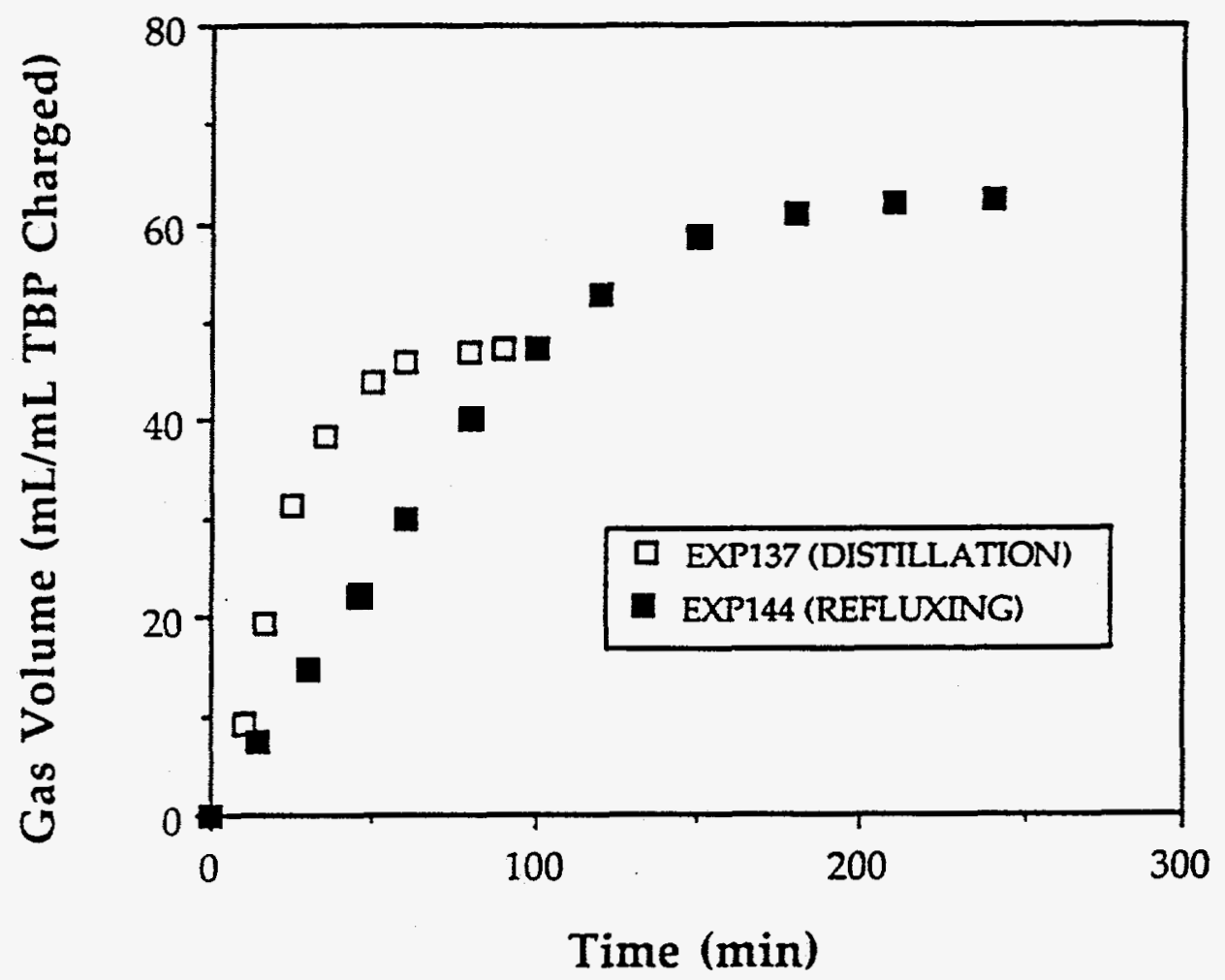


Fig. O-4 Effect of Distillation/Refluxing (SP,CP.6M,150 $\left.{ }^{\circ} \mathrm{C}\right)$

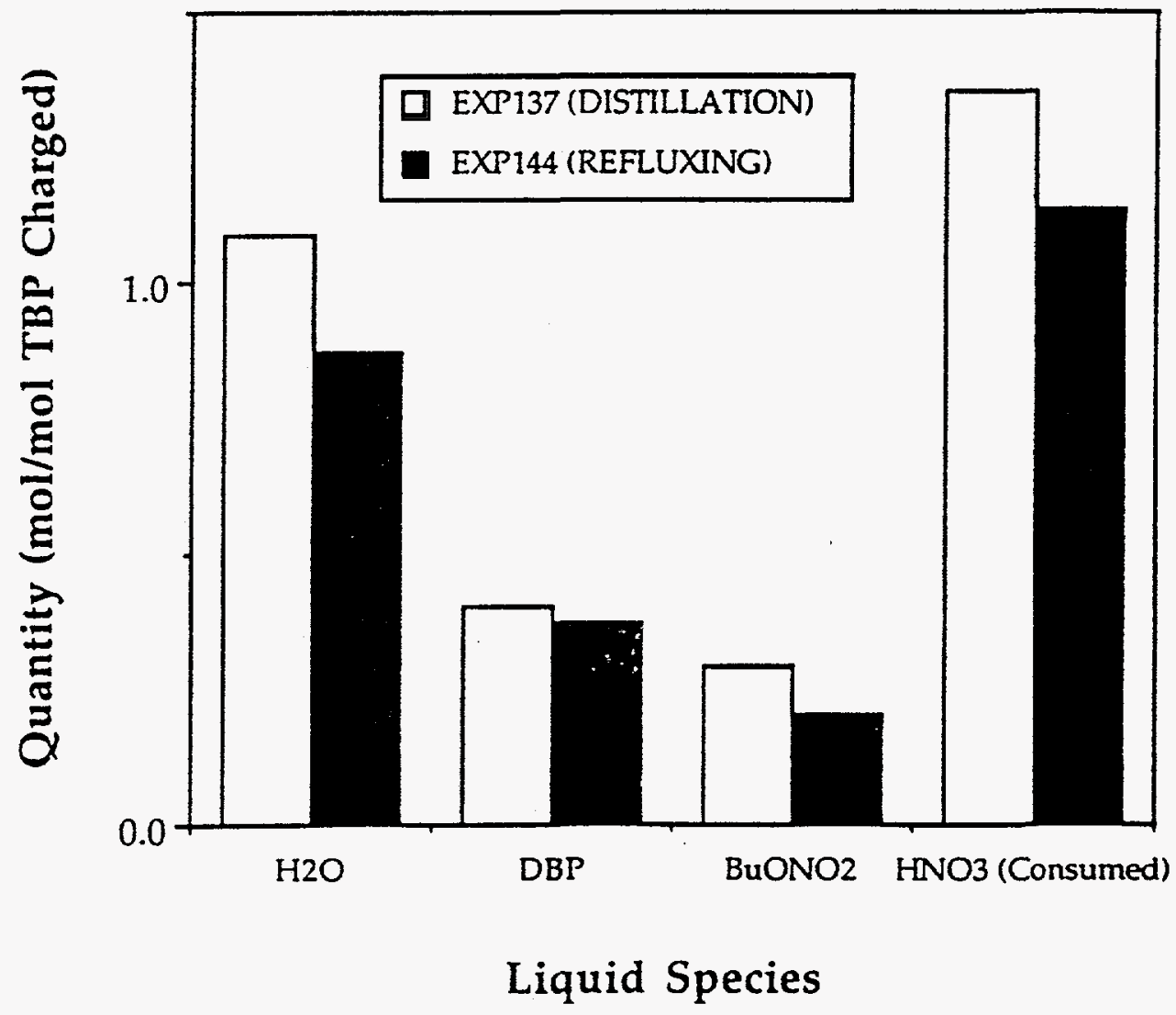




\section{ATTACHMENT \#1}

Georgia Tech's Technical Proposal 
WSRC-RP-95-259

RFP \#94060EQ

Proposal to

WESTINGHOUSE SAVANNAH RIVER COMPANY

Through the

Education, Research and Development Association (ERDA) of Georgia Universities

For

THERMAL DECOMPOSITION OF NITRATED TRIBUTYL PHOSPHATE

Principal Investigator:

Co-Investigators:

Proposal Period:

Total Cost of Proposal:

Principal Participants:

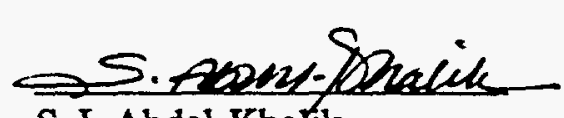

S. I. Abdel-Khatik

(404) 894-3719
Dr. S. I. Abdel-Khalik, Southern Nuclear Professor School of Mechanical Engineering

Dr. E. K. Barefield, Professor

School of Chemistry

Dr. D. W. Tedder, Associate Professor

School of Chemical Engineering

6 Months

$\$ 74,993$

Reguired Ofganizationgl Approval:

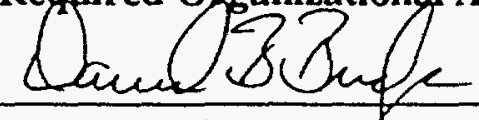

David B. Bridges, Coptracting Officer Georgia Tech Research Corporation Atlanta, GA 30332-0420

(404)894-4817

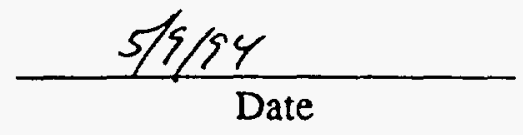


WSRC-RP-95-259

RFP \#94060EQ

\section{STATEMENT OF SCOPE \\ THERMAL DECOMPOSITION OF NITRATED TRIBUTYL PHOSPHATE}

\subsection{SCOPE OF WORK}

\subsection{Introduction}

Contact between tributyl phosphate (TBP) and aqueous solutions of nitric acid and/or heavy-metal nitrate salts can lead to exothermic reactions of explosive violence. Even though this operation has routinely been performed safely for decades as an intrinsic part of the Purex separations process, four such major explosions are known to have occurred. The most recent explosion occurred at the Tomsk-7 separations facility in Siberia, in April, 1993. That explosion destroyed part of the unreinforced masonry walls of the canyon-type building in which the process was housed, and allowed the release of a significant quantity of radioactive material.

The Tomsk incident raised questions about the effects of a similar explosion occurring in SRS separations processes. In order to assess the severity of such explosions, it is necessary to estimate the yield of volatile flammable gases that may be produced. To this end, this work will provide data to characterize the chemistry of decomposition reactions involving nitrated tributyl phosphate (TBP), and its derivatives.

\subsection{Objectives}

This study will focus on analytical techniques to accurately and reproducibly provide material balance information describing the decomposition products in both the gaseous and liquid phases. The ultimate goal of the experiments is to provide the basis for estimating the energetics of the organic-nitrate reactions in real systems. The research will provide estimates of the yield of volatile flammable gases that may escape from the process vessel and represent an ignition hazard.

\subsection{Statement of Work}

1. Design, construct, and instrument an experimental apparatus to determine the material balance in the partial thermal decomposition of nitrated tributyl phosphate (TBP).

2. Conduct single phase and two-phase thermal decomposition experiments as outlined in the experimental protocol described in Appendix A.

3. Provide progress reports and a final report summarizing the findings of the investigation. 
RFP \#94060EQ

\subsection{Reporting}

Monthly status reports shall be issued to the WSRC technical representative by the tenth of each month.

\subsection{Deliverables}

In addition to the monthly status reports, the following reports will be provided:

1. A progress report summarizing the results of the single phase thermal decomposition experiments will be provided after two months of project initiation.

2. A final report summarizing the results of the investigation should be provided to WSRC upon conclusion of the project.

Both the progress report and final report shall meet the requirements of WSRC's "Specification for Technical Information".

\subsection{RESPONSIBILITIES}

The Process Safety Technology Section of SRTC will provide any technical information on prior TBP decomposition experiments and related literature to the Principal Investigator.

\subsection{PERIOD OF PERFORMANCE}

The contract will be for a period of six months after the Task Order Award date.

\subsection{LEVEL OF EFFORT}

See attached budget explanation for the required level effort and associated costs.

\subsection{QUALITY ASSURANCE PLAN}

The checklist requirements for the Quality Assurance Plan attached to the RFP (\#94060) are acceptable.

The Thermal Decomposition of Nitrated Tributyl Phosphate project has three related subtasks as specified in Section 1.3 above. The Principal Investigator, Dr. S. I. AbdelKhalik, will coordinate all three subtasks. The PI will achieve the quality assurance by transmitting all requirements to the co-investigators and all other participants through weekly project review meetings. Decisions and actions taken during these meetings will be reported in the monthly progress reports. 
Tasks have been planned based on preliminary discussions between the PI, the Coinvestigators, and the WSRC Technical Representative. Progress will be monitored regularly and reported monthly.

Due to the specialized nature of the software used and developed for data collection and analysis, the PI will rely on the evaluation and effort of each researcher. The PI will establish that all researchers are aware of all requirements and will actively pursue the compliance of all requirements.

Records of procurements are maintained by existing staff within the Georgia Institute of Technology, at an Institute wide and School (department) level. The PI will maintain access to these records. In addition, a file will be maintained of all purchases by the PI.

Any equipment or material affecting test results will be documented by the PI. Similarly, technical task records, task data, and logbooks/laboratory notebooks will be generated by each researcher. In order to promote full use of these record keeping devices, the weekly progress meetings will compare activities planned and undertaken to the documentation supplied for task records. These reviews will be based on group participation to stimulate technical communication among researchers and to give the most thorough review.

Logbooks will be maintained by each researcher. One of the Co-investigators, Dr. D. W. Tedder, will review logbooks and generate monthly entries into an index to the history of the overall task.

Logbooks shall be bound and shall have numbered pages. Entries shall be legible in ink with no erasers. Any changes or corrections to entries shall be made by drawing a single line through the erroneous entry and having the corrections entered adjacent to the error, dated and initialed. If the reason for the change is significant and not obvious, then the reason shall be recorded next to the change as well. Pages of the logbook shall be filled consecutively. Spaces and pages left blank shall be crossed out so that there is no doubt about whether data should have been recorded or is missing. Supporting documentation (e.g., data sheets, drawings, computer printouts) shall be inserted into the logbook when practicable, or otherwise referenced. Logbook entries shall be signed and dated by the person making the entry. If only one person is making entries into a logbook, that person need only sign and date the logbook at the end of the operation. If however, more than one person is making entries into the same logbook during inspection or task, each person shall sign and date his entries upon completing them.

Monthly reports will be generated by the PI based on a survey of the researchers, the account records, and the weekly research meetings. Technical reports to WSRC will be coordinated by the PI. The PI will establish a consistent format for all sections and will be responsible for prompt delivery. All reports, including the final report, shall be reviewed and approved by the PI as evidenced by signature and title. The Technical Representative is the point of contact for WSRC review/approval and distribution. 
WSRC-RP-95-259

RFP \#94060EQ

(This page intentionally left blank.) 
WSRC-RP-95-259

RFP \#94060EQ

(This page intentionally left blank.) 
WSRC-RP-95-259

RFP \#94060EQ

(This page intentionally left blank.) 
WSRC-RP-95-259

RFP \#94060EQ

Appendix A

EXPERIMENTAL PROTOCOL

\section{THERMAL DECOMPOSITION OF NITRATED TRIBUTYL PHOSPHATE}

\section{Sample Preparation}

Reagent grade nitric acid, TBP and distilled water will be used. Equal volumes of pure TBP and $9 \mathrm{M}$ nitric acid solution (e.g., $100 \mathrm{~mL}$ samples of each) will be gently mixed at room temperature to yield approximately a $6 \mathrm{M}$ aqueous phase in equilibrium with an organic phase containing about $3 \mathrm{M}$ HN03. Equilibration will be carried out at room temperature over 24 hours with gentle stirring of the aqueous phase. Afterwards this mixture will be sealed and used for decomposition tests within 48 hours of preparation.

Samples for decomposition experiments (either single or two phase tests) will be removed volumetrically (e.g., $1 \mathrm{~mL}$ samples). Both phases will be subjected to chemical analysis.

Mixture samples will be saved for eventual decomposition tests on aging extract. Initial experiments will be conducted using freshly equilibrated samples that have not been aged more than 48 hours. Aged samples will be periodically subjected to chemical analysis, especially to monitor TBP decomposition.

\section{Extract Decomposition (Single Phase Tests)}

Constant pressure ( $1 \mathrm{~atm}$ ) experiments will be completed by gently heating extract for varying lengths of time at specified temperatures. Liquid compositions will be analyzed at the start and end of each experiment. All residual liquid will be transferred into a graduated cylinder to inspect for multiple phases and weighed. Phase volumes will be noted and samples taken for chemical analysis. Decomposition gas volumes will be measured at one atmosphere at room temperature. During a test, approximate volume versus time data will be obtained. Final gas compositions will be determined using chromatography.

Several constant volume experiments will be completed in small 316 stainless steel pressure bombs first to characterize decomposition rates and verify that decomposition at constant pressure can be performed safely in glassware. In these tests, steel pressure bombs will be gradually heated while container pressure is measured using a baratron. The rate of pressure rise will be measured as a function of temperature and heating rates.

Liquid phase samples will be analyzed at the start and end of each experiment. Gas compositions will be determined by chromatography. After depressurization, all residual liquid will be transferred into a graduated cylinder to inspect for multiple phases and weighed. Phase volumes will be noted and samples taken for chemical analysis. 


\section{Two Phase Decomposition Tests}

These experiments are similar to those planned for the Single Phase system with one difference. Equal volumes of each phase (see Step 1 above) will be removed from freshly equilibrated mixtures and subjected to decomposition.

Constant pressure ( $1 \mathrm{~atm}$ ) experiments will be completed by gently heating extract for varying lengths of time at specified temperatures. Liquid compositions will be analyzed at the start and end of each experiment. All residual liquid will be transferred into a graduated cylinder to inspect for multiple phases and weighed. Phase volumes will be noted and samples taken for chemical analysis. Decomposition gas volumes will be measured at one atmosphere and room temperature. During a test, approximate volume versus time data will be obtained. Final gas compositions will be determined using chromatography.

Several constant volume experiments will be completed first in small 316 stainless steel pressure bombs to characterize decomposition rates and verify that decomposition at constant pressure can be performed safely in glassware. In these tests, steel pressure bombs will be gradually heated while container pressure is measured using a baratron. The rate of pressure rise will be measured as a function of temperature and heating rates.

Liquid phase samples will be analyzed at the end of each experiment. Gas compositions will be determined by chromatography. After samples have been depressurized, all residual liquid will be poured into a graduated cylinder to inspect for multiple phases by volume and then weighed. Samples will be taken of each residual phase for analysis.

\section{Liquid Phase Analysis}

Liquid phases (both extracts and reacted phase residuals) will be analyzed several ways. Nitric acid concentrations will be titrated using $0.1 \mathrm{M} \mathrm{NaOH}$ calibrated with potassium biphthalate. Bromthymol blue indicator will be titrated to a pH of 7 .

Water concentrations will be measured using Karl Fischer titration and proton NMR.

TBP and organophosphate degradation product concentrations will be measured using high pressure liquid chromatography (HPLC).

\section{Gas Phase Analysis}

Decomposition product volumes will be measured at one atmosphere and room temperature to obtain a quantitative relationship between gaseous yields per gm of extract. Gas chromatography will be used to determine the composition of decomposition products. 
WSRC-RP-95-259

\section{ATTACHMENT \#2}

Georgia Tech's Monthly Progress Reports 


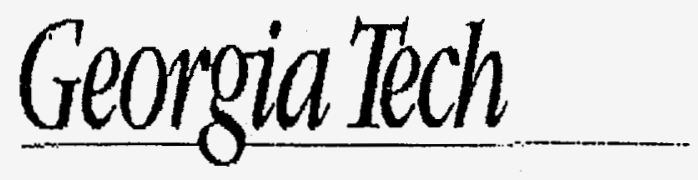

9. I. Abdel-Khalik

Southern Nuclear Distinguished Unfessor

Nuclear Engineering Program
THE GEORGE W. WOODRUFR SCHOOL OF MECHANICAL ENCINEERING

Georgla lastitute of 'Technology

Allane, Georgia 30132-12405

USA

August 9, 1994

\section{Mr. D. T. Paddleford}

Safety Technology Department

Westinghouse Savannah River Company

1991 S. Centennial Avenue

Aiken, South Carolina 29803-7657

Dear Don:

Attached is the first monthly status report for our ERDA contract on the thermal decomposition of nitrated TBP. Work is progressing on schedule. As specified in the task order, we expect to complete the single phase experiments by mid September.

Please feel free to call me if you have any questions.

Sincerely.

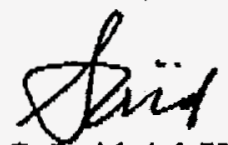

s. I. Abdel-Khalik

\section{SIAK/bC}

cc: Dr. E. K. Barefield

Dr. D. W. Tedder

Dr. R. A. Karam, ERDA

Mr. Pete Dawkins

OCA/Georgia Tech (2) 
Send Monthly Status Report by the 10th of each month to:

Dr. Ratib Karam, ERDA

Neely Nuclear Research Center

900 Atlantic Drive

Atlanta, GA 30332-0405

University Georgia Institute of Technology

PI Dr.S.I. Abdel-Khalik

Project Title Thermal Decomposition of Nitrated Tributyl Phosphate

WSRC Technical

Period of Performance: From Z/11/94 To 1/31/95 Representative_Don Paddleford

A. Project Accomplishments:

See attached.

B. Milestones achfeved (Based on those identified In the Task Order):

Work is progressing on schedule. As specified in the Task Order, single-phase experiments will be completed two months after project initiation (9/11/94).

C. Problems Encountered:

None 


\title{
ERDA TASK ORDER \#94054
}

\section{Thermal Decomposition of Nitrated Tributyl Phosphate}

\author{
Monthly Status Report
}

(July 11, 1994 - August 10, 1994)

Reagent grade nitric acid, TBP and distilled water were equilibrated to yield a TBP phase in equilibrium with approximately $6 \mathrm{M}$ nitric acid. Titration and proton NMR analysis indicated that the equilibrated organic phase contained about $3 \mathrm{M} \mathrm{HNO} 3$ and $2 \mathrm{M} \mathrm{H} 20$.

Single phase decomposition experiments were conducted at 1 atm total pressure and constant temperatures of 130,150 and $180^{\circ} \mathrm{C}$. Five $\mathrm{mL}$ of equilibrated TBP were maintained at the respective temperatures for periods of time up to six hours. The gas volumes evolved from these samples were measured at standard temperature and pressure over the time of the experiment (Figure 1). The nonvolatile residuals from each experiment were analyzed using proton NMR, and titrated for acidic proton content. Phosphorous NMR analysis will be performed. Figure 2 shows typical NMR spectra for the residual liquid of the $180^{\circ} \mathrm{C}$ experiment.

The NMR results suggest that at high temperatures (c.g. $180^{\circ} \mathrm{C}$ ), significant anounts of TBP will undergo hydrolysis to produce dibutyl phosphate, along with small amounts of monobutyl phosphate. At low temperatures, however, (e.g. $130^{\circ} \mathrm{C}$ ), much of the residual liquid was unreacted TBP. Gas analysis has not yet been completed, but we suspect that the results will primarily show the presence of nitrogen oxides and, secondarily, small amounts of TBP degradation products.

Isothermal gas product formation from TBP extract appears to consist of: (1) an induction period, (2) a period of constant gas formation, and (3) an approach to equilibrium in which gas generation stops. Our results show that the induction period decreases with increasing temperature and that the rate of gas formation increases with temperature (Figures 1 and 3).

A single experiment at $150^{\circ} \mathrm{C}$ was completed in which water and nitric acid saturated TBP was equilibrated with zirconyl nitrate hydrate solid. The resulting TBP extract was then reacted at constant pressure and temperature of $1 \mathrm{~atm}$ and $150^{\circ} \mathrm{C}$, respectively. The observed induction period was much shorter than without $\mathrm{Zr}$ at the same temperature (Figure 3). The rate of gas production was also greater with $\mathrm{Zr}$ than without, however, the total amount of gas produced was slightly reduced (Figure 4). These results suggest that $Z r$ catalyzes gas formation from TBP extract, and that measuring gas evolution without the presence of $\mathrm{Zr}$ or other similar catalysts (e.g., U, Pu or Th) may significantly underestimate risk. 
Gas Produced as Function of Time

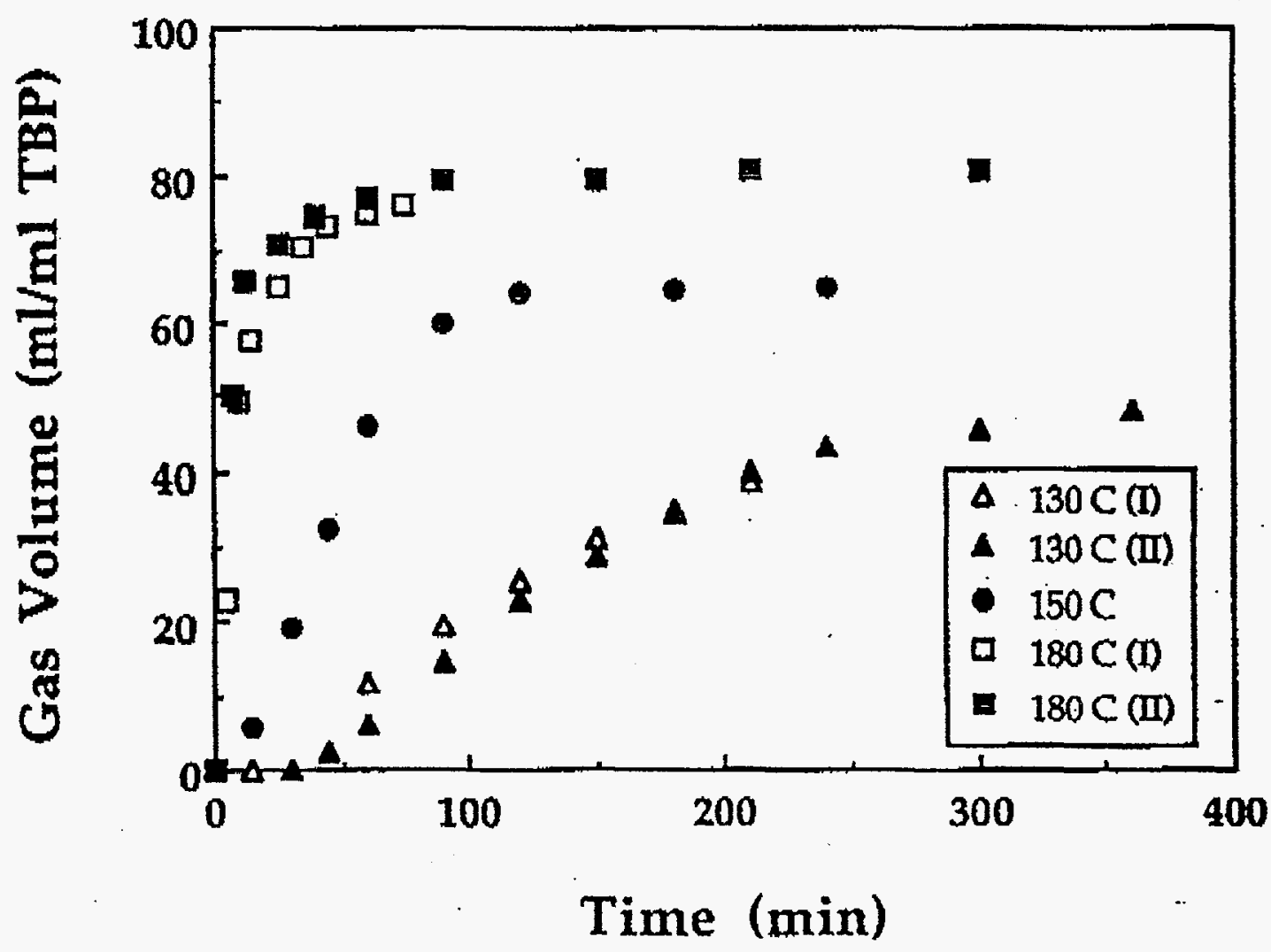

Flgure 1: Cumulative Gas Production from Organic Phase Decomposition at Various Temperatures 


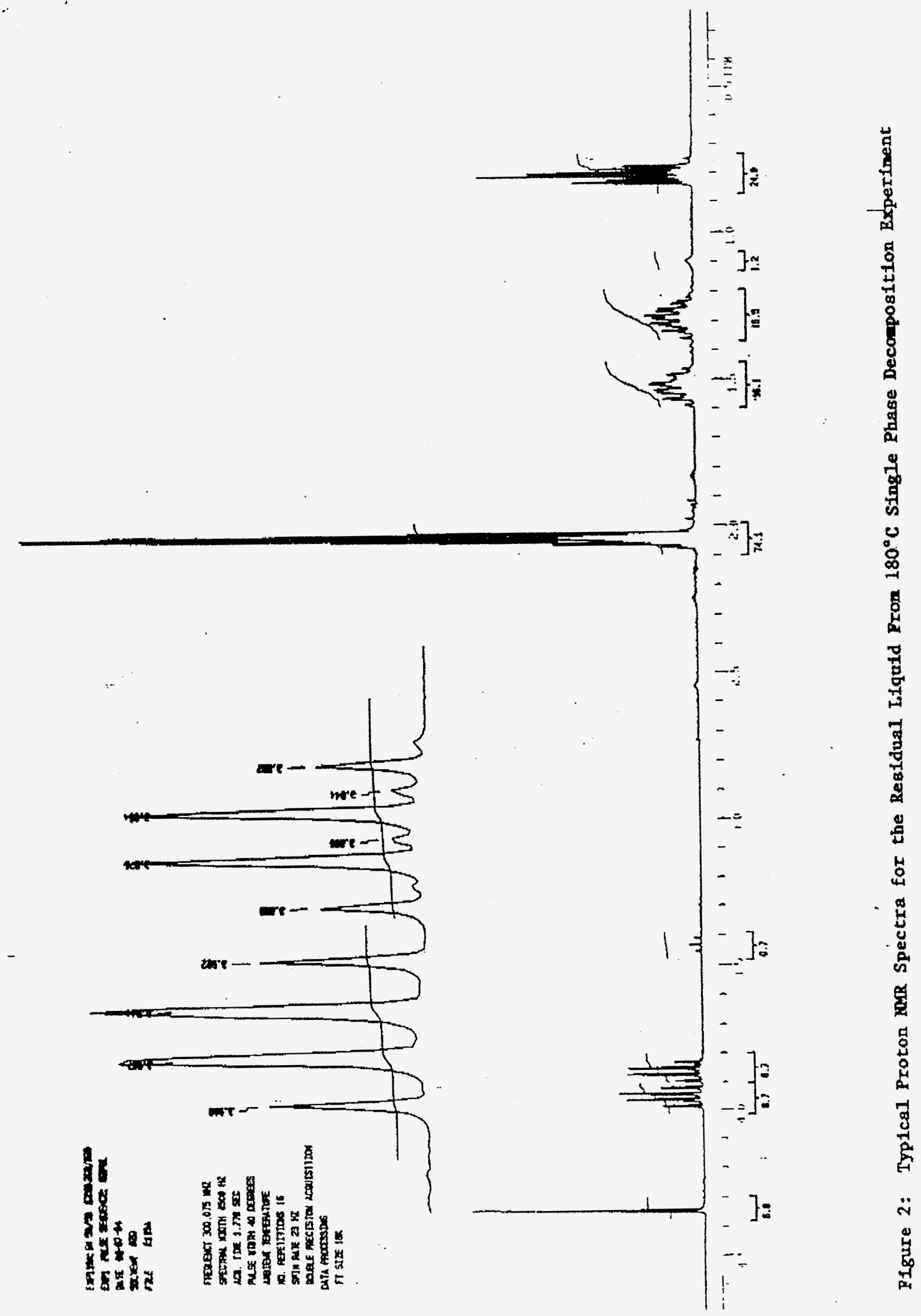




\section{Linear Part of Volume-Time Plots}

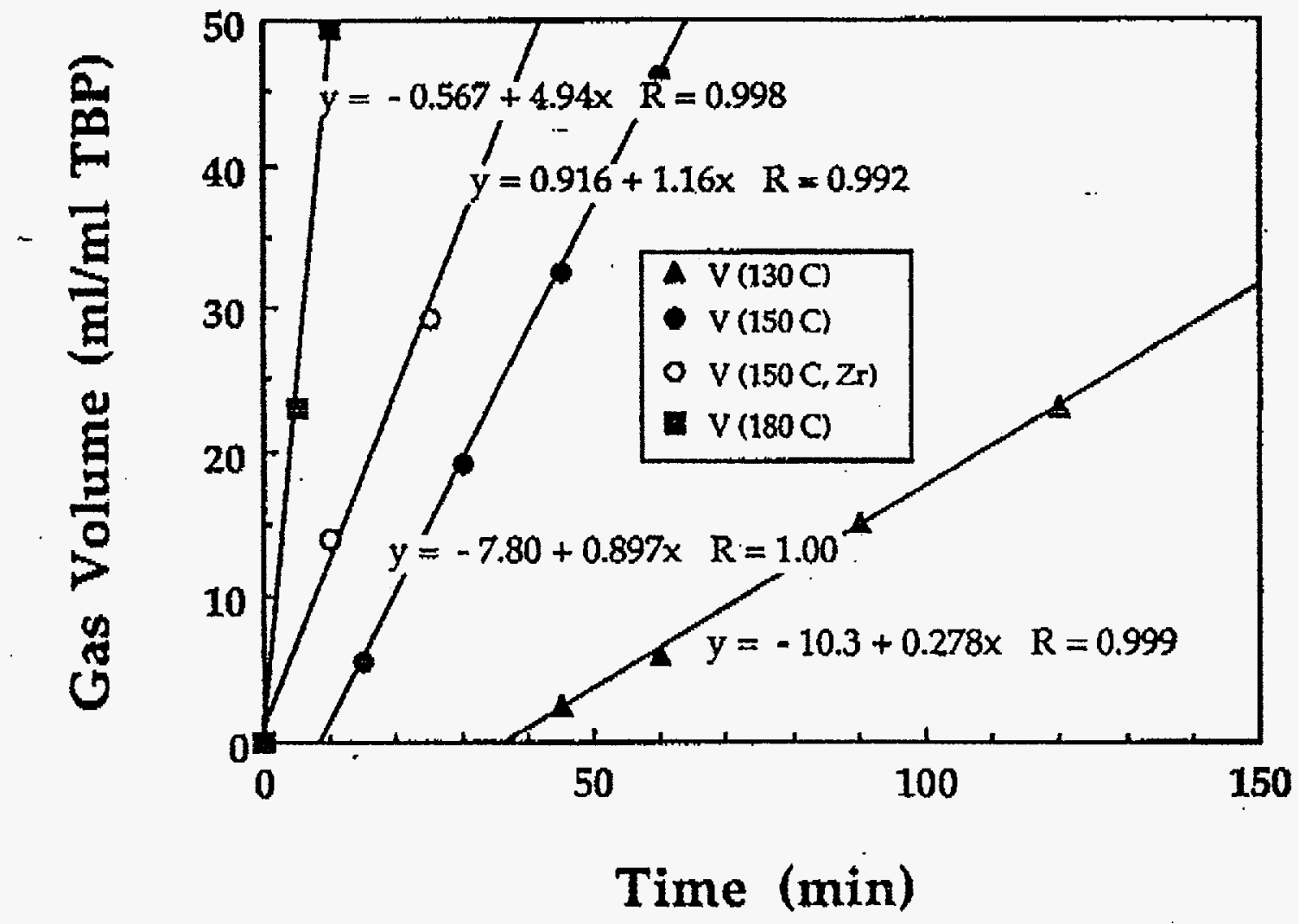

Figure 3: Gas Production Rates From Organic Phase Decomposition at Various Temperatures 
Gas Produced with/without Catalyst

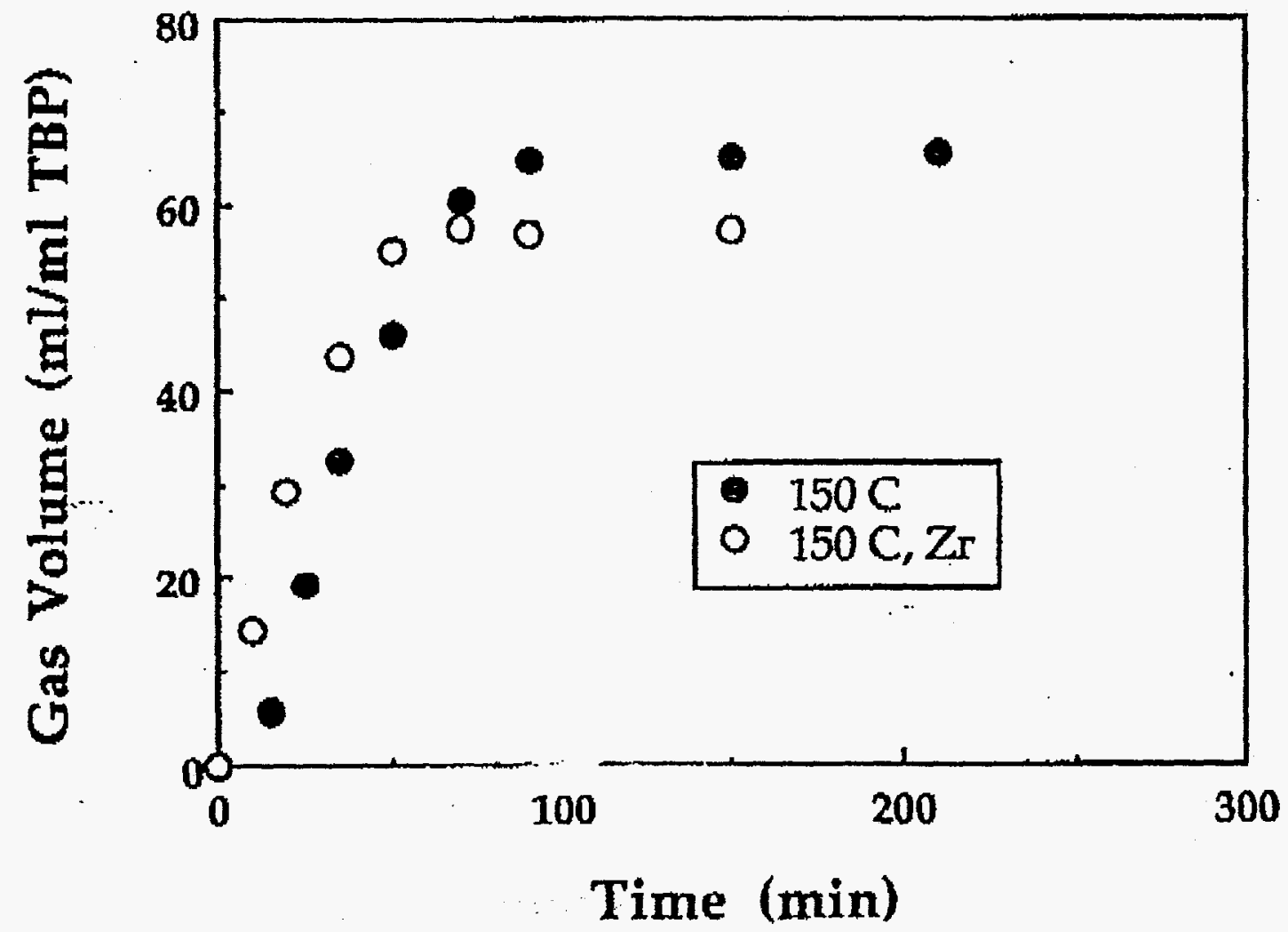

Figure 4: Effect of Zirconyl Nitrate on Gas Production from Organic Phase Decomposition at $150^{\circ} \mathrm{C}$ 
GTRSR-021

\title{
THERMAX DECOMPOSITION OF NITRATED TRIBUTYL PHOSPHATE
}

\author{
Progress Report
}

(July 11, 1994 - September 9. 1994)

Subraitted to

Westinghouse Savannah River Company

(Task Order \#94054 of ERDA Subcontract \#AA46420T)

\section{By}

Y. Hou' ${ }^{3}$ E. K. Barefield ${ }^{2}$, D. W. Tedder ${ }^{3}$, and S. I. Abdel-Khalik ${ }^{1}$

Georgia Institute of Technology

September 9, 1994

\footnotetext{
School of Mechanical Engineering

${ }^{2}$ School of Chemistry

${ }^{3}$ School of Chemical Engineering
} 


\section{Executive Summary}

Gas production from single-phase mixtures of tri-n-butyl phosphate (TBP), water, and nitric acid was studied at constant pressure and under reflux conditions as a function of temperature (130, 150 and $180^{\circ} \mathrm{C}$ ), acid concentration (nominally $3 \mathrm{M}$ and $6 \mathrm{M}$ ), and in the presence and absence of $\mathrm{Zr}^{4+}$ (saturated). The rate of gas production increased with temperature, with increased acid concentration and in the presence of $\mathrm{Zr}^{4+}$. The quantity of gas per unit volume of sample increased with temperature and acid concentration but was slightly less in the presence of $\mathrm{Zr}^{4+}$. Dibutyl phosphate (DBP) and monobutyl phosphate (MPB) were observed as products of the decomposition reactions in all cases except in runs involving $\mathrm{Zr}^{\text {14}}$. Small amounts of 1-butanol and butyl nitrate were also observed in the residue. In the presence of $\mathrm{Zr}^{4+}$ the residue from the decomposition was a solid, which was poorly soluble in organic solvents. Phosphates other than TBP were not positively ideatified for runs involving $\mathrm{Zr}^{4+}$. A similar series of experiments has been recently initiated in which volatiles were distilled from the reactor as they are formed. Substantial amounts of butyl nitrate were collected under these conditions.

\section{Experimental Procedures}

\section{Sample and Standards Preparation}

Samples were prepared using the highest purity reagents that were available. Tri- $n$-butyl phosphate (TBP), 99\%, was obtained from Aldrich Chemical Co. and used as received. Reagent grade nitric acid was diluted with distilled water as required. All solutions were purged with argon and stored in sealed vessels under an argon atmosphere.

Fisher standard $1 \mathrm{M}$ and $0.2 \mathrm{M} \mathrm{NaOH}$ were used to determine acid concentrations using bromthymol blue as the indicator. Nominal $9 \mathrm{M} \mathrm{HNO}_{3}$ was prepared using concentrated reagent grade acid diluted with distilled water. Titration indicated the actual acid concentration was $8.9 \mathrm{M}$. This solution was used for all equilibrations with TBP to generate 3 M TBP solutions that are described in this report. Reagent grade nitric acid was used for equilibrations to generate the $6 \mathrm{M}$ equilibrated TBP solutions.

Concentrations of water in equilibrated samples and in products and residues have been determined by Karl Fisher titration.

Equilibrations with TBP and $9 \mathrm{M}$ acid were done by stirring equal volumes of each component. Equilibrations of TBP and concentrated acid (16 M) were done using one part of TBP and three parts of the concentrated acid. The two phase system was purged with argon for 30 minutes, sealed and allowed to cquilibrate at room temperature with gentle stirring. Titrations of aliquots taken from the aqueous phase as a function of time indicated that equilibrium was achieved after 24 hours of mixing. Equilibrations with the $9 \mathrm{M}$ acid gave acid concentrations of $3.00 \mathrm{M}$ in the organic phase and $6.3 \mathrm{M}$ acid in the aqueous phase. Equilibrations with concentrated acid gave acid concentrations of $5.7 \mathrm{M}$ in the organic phase and $15 \mathrm{M}$ acid in the aqueous phase. Portions of all equilibrated solutions have been stored at room temperature and at $50^{\circ} \mathrm{C}$ under argon since their preparation to determine the effects of aging. 


\section{Procedure for Constant Pressure Decompositions}

Decomposition reactions of equilibrated TBP/nitric acid samples were conducted in the apparatus shown in Figure 1, which was modified to incorporate sampling valves for obtaining gas samples for mass spectroscopy and infrared spectroscopy. This apparatus allows for volumetric determination of gas production up to $600 \mathrm{~mL}$. In runs where the volatiles were collected, the condenser was repositioned to allow for distillation rather than reflux and a receiver placed between the condenser and gas measurement system. Runs done under reflux conditions had sample temperatures that were about $15^{\circ} \mathrm{C}$ less than the bath temperature. The reaction vessel was either a $50 \mathrm{~mL}$ or $100 \mathrm{~mL}$ flask, which was immersed in an oil bath up to the neck of the flask. In some runs a three-necked flask was used so that a thermocouple could be inserted into the sample.

Experiments were conducted by placing a known volume of equilibrated TBP mixture into a tared flask and the mass of the sample determined by difference. The flask was fitted to the condenser and the system evacuated and back-filled with argon several times. A preheated oil bath was raised such that the flask was immarsed to the neck. The system was vented to the atmosphere for a short time ( $<2 \mathrm{~min}$ ) to maintain aimospherie pressure while the temperature equilibrated. At this point the time was recorded and periodic measurements of the volume of gas produced were made. At the completion of a run, gas samples were removed for mass and infrared spectroscopic analysis. The oil bath was lowered and, after cooling to room temperature, the flask was removed, sealed (with tared stopper), and cleaned with solvent. At this point the mass of the residue was determined and then samples were removed for NMR analysis and for titration of acid and water content.

\section{Results}

\section{Gas Production}

Experiments were conducted in $100 \mathrm{~mL}$ reaction flasks under reflux conditions at 130,150 and $180^{\circ} \mathrm{C}$ (bath temperature) with TBP containing $3 \mathrm{M}$ acid and at 130 and $180^{\circ} \mathrm{C}$ with TBP containing $6 \mathrm{M}$ acid. Gas production in $\mathrm{mL}$ per $\mathrm{mL}$ of sample are presented in Figure 2 for the former runs. The rate of gas production and the total amount of gas produced increased with temperature. Figure 3 gives a comparison of gas production at $150^{\circ} \mathrm{C}$ for TBP containing $3 \mathrm{M}$ and $6 \mathrm{M}$ acid. Both the rate and amount of gas produced increased at the higher acid concentration. Induction periods are evident for nuns at lower temperatures at both acid concentrations. It must be noted that the actual sample temperature is somewhat less (approximately $15^{\circ} \mathrm{C}$ ) than the bath temperature as a result of heat loss due to reflux of the volatile products.

Figure 4 shows the temporal production of gas at $150^{\circ} \mathrm{C}$ for TBP containing 3 and $6 \mathrm{M}$ acid that has been saturated with $\mathrm{ZrO}\left(\mathrm{NO}_{3}\right)_{2} \times \mathrm{xH}_{3} \mathrm{O}$. Data under the same conditions without added $\mathrm{Zr}^{4+}$ is shown for comparison. The rate of gas production is greater in the presence of $\mathrm{Zr}^{4+}$ but the total amount of gas is slightly less. The induction period for TBP with $3 \mathrm{M}$ acid is reduced in the presence of $\mathrm{Zr}^{1+}$

The existence of an induction period is shown more clearly in Figure 5, which gives plots of gas production during the period of constant rate of evolution for several of the decomposition 
experiments described above. The effect of temperature, acid concentration and presence of $\mathrm{Zr}^{4+}$ are obvious.

Recently experiments have been undertaken under distillation conditions; that is, volatiles were condensed as formed and removed from the reaction vessel. Gas production appears to increase under distillation conditions, as shown in Figure 6, but it must be noted that the actual sample temperature is greater under distillation conditions. Thermocouple measurements indicate that the sample temperature is about $2^{\circ} \mathrm{C}$ less than the bath temperature under distillation conditions. Further experiments are planned to determine what factors are important to gas production. Analysis of the distillate indicate that it is predominantly a mixture of water and butyl nitrate

When the reaction vessel volume was reduced from 100 to $50 \mathrm{~mL}$ the volume of gas produced per $\mathrm{mL}$ of sample under reflux conditions decreased, as shown in Figure 7. Although this requires further investigation, we believe that this is a result of better heat transfer and more uniform heating in the larger flask. Temperature differences may be the main factor but the rate at which the sample is heated may also be a factor.

-

Gas Analysis

Analyses of gas phases have only been recently undertaken. Mass spectra indicate that carbon dioxide, carbon monoxide and NO are present when gas production ceases. Although the gas produced initially is orange brown, which suggests the presence of $\mathrm{NO}_{2}$, no confirmatory evidence is yet available. The infrared spectra of two samples suggest the presence of some hydrocarbon (C-H stretching absorption) but this is not supported by the mass spectra. Further work is planned.

\section{Residuals Analysis}

The mass of the material remaining in the reaction flask after gas evolution ceased has been determined for most reactions and analyses of acid content done by titration. ${ }^{\mathrm{I}} \mathrm{H}$ and ${ }^{31} \mathrm{P}$ NMR spectroscopies have been used to further determine the composition of these aamples. IH spectra are useful for determining hydrolysis products (butanol and butyl nitrate) but the butyl group resonances of TBP, DBP and MBP have such similar chemical shifts that they cannot be reliably determined using this method. ${ }^{31} \mathrm{P}$ NMR spectroscopy on the other hand provide a convenient means of determining these compounds.

As an example of the method, a $5 \mathrm{~mL}(5.1 \mathrm{~g})$ sample of TBP containing $3 \mathrm{M}$ acid yielded 205 $\mathrm{mL}$ of gas $(1 \mathrm{~atm})$ under reflux (bath temperature $150^{\circ} \mathrm{C}$ ) in $50 \mathrm{~mL}$ flask and gave a residue of 4.7 g. Titration with $\mathrm{NaOH}$ indicated that the total acid concentration in the residue was $2.6 \mathrm{M}$. The ${ }^{31} \mathrm{P}$ NMR spectrum (Figure 8) indicated that there was both DBP and MBP as well as unreacted TBP in the residue. The TBP:DBP:MBP ratios were $3: 3: 1$. The water contents of the starting sample and the residue were the same at $1.4 \mathrm{M}$. The ' $\mathrm{H}$ NMR spectrum shows the presence of small amounts of butyl nitrate in addition to the mixture of phosphate esters.

The presence of $\mathrm{Zr}^{4+}$ in TBP samples appears to affect the physical and chemical properties of the residue. Whereas the uncatalyzed reaction residues are viscous liquids, those done with added 
$\mathrm{Zr}^{4+}$ are waxy solids that are insoluble in most organic solvents but slightly soluble in DMSO. The "P NMR spectra (see Figure 9 for an example) of these residues suggest the presence of TBP but do not show the presence of DBP or MBP. There is, however, a broad resonance upfield whose identity is not yet known.

As indicated above, under distillation conditions a mixture of water and butyl nitrate distills from the reaction flask. As expected the amount of residue is less under distillation conditions. ${ }^{31} p$ NMR spectra of TBP containing $3 \mathrm{M}$ acid nu at $150^{\circ} \mathrm{C}$ under both conditions indicate that less hydrolysis of TBP appears to occur under distillation conditions.

Table 1 give mass balances for several of the runs for which data are most complete. It is expected that elemental balances will be provided when chemical analyses are completed. 


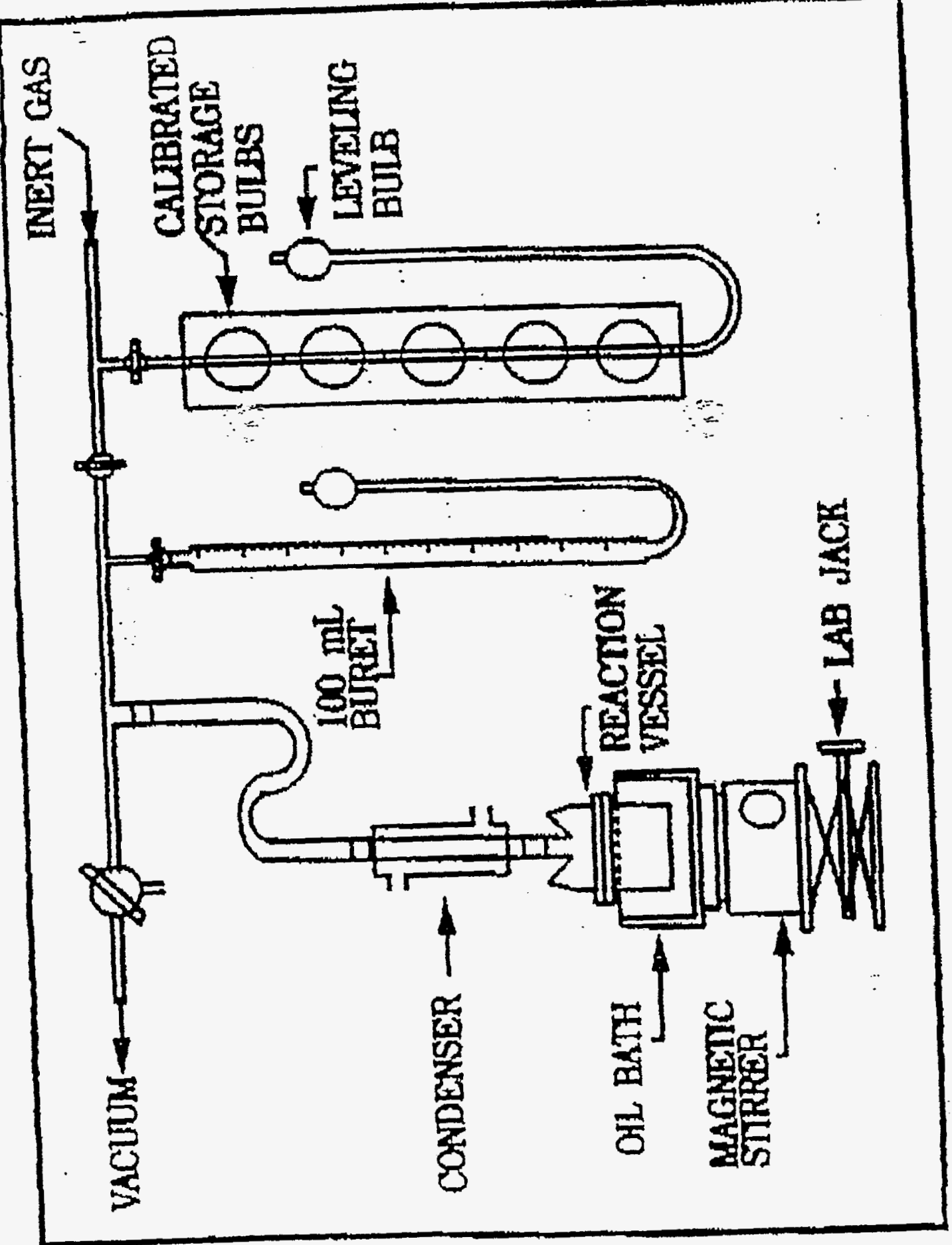

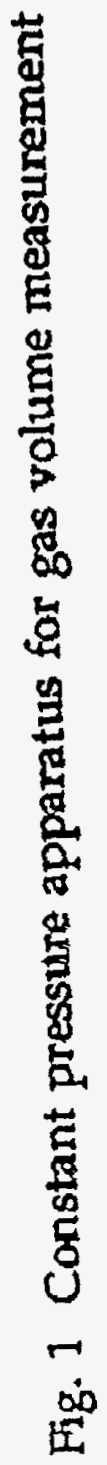




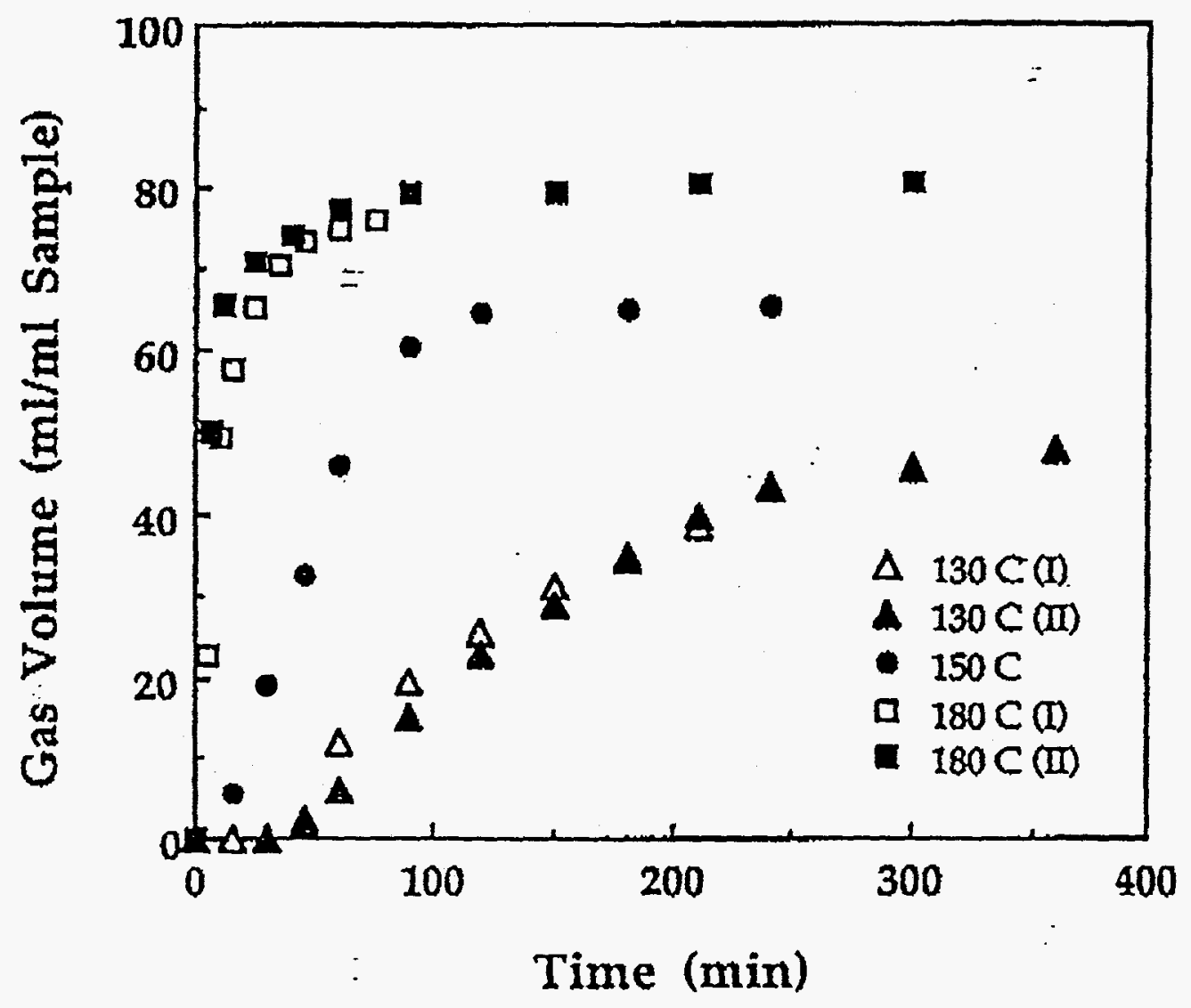

Fig. 2 Effect of temperature on gas production 


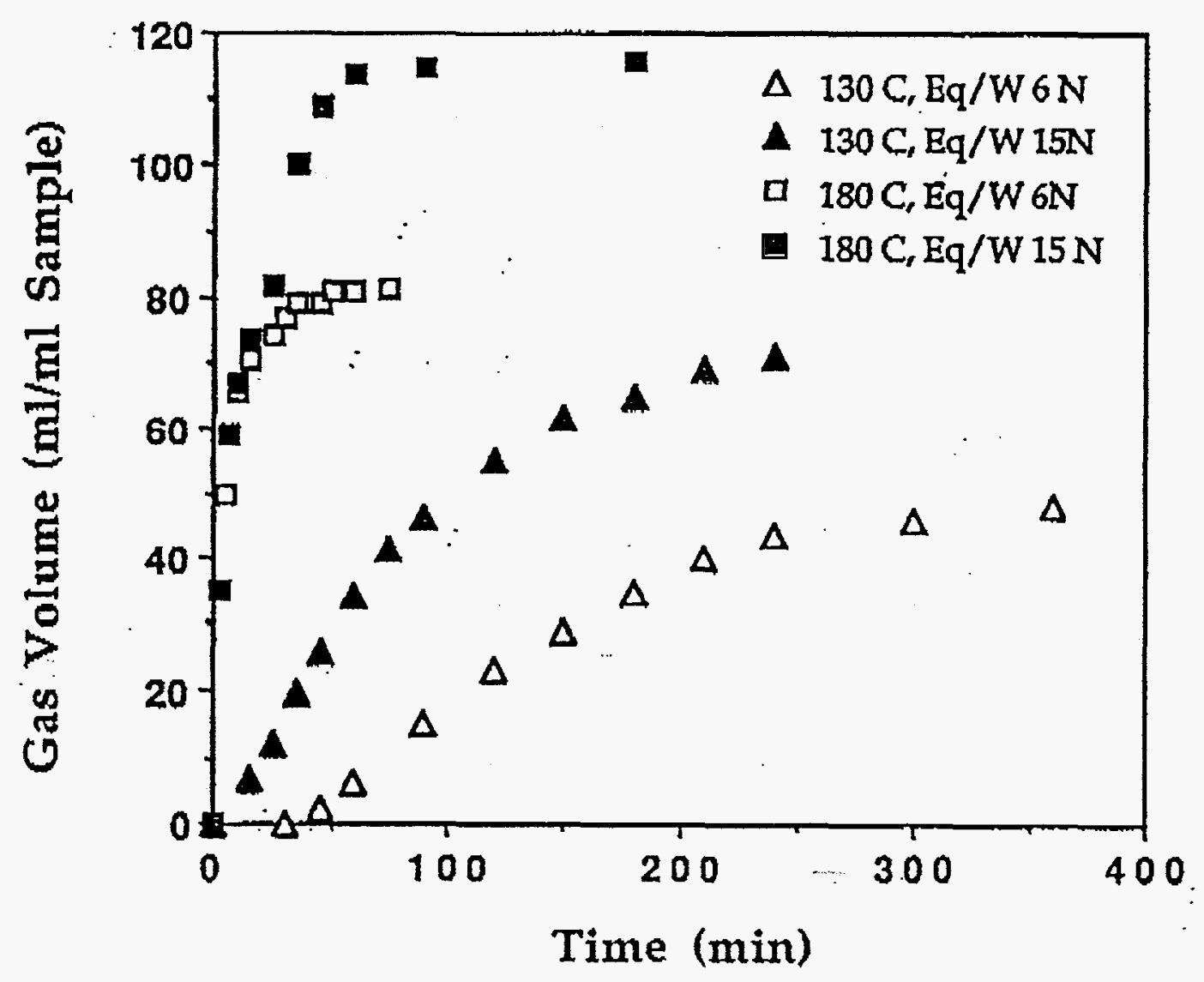

Fig. 3 Effect of temperature and acid concentration on gas production 

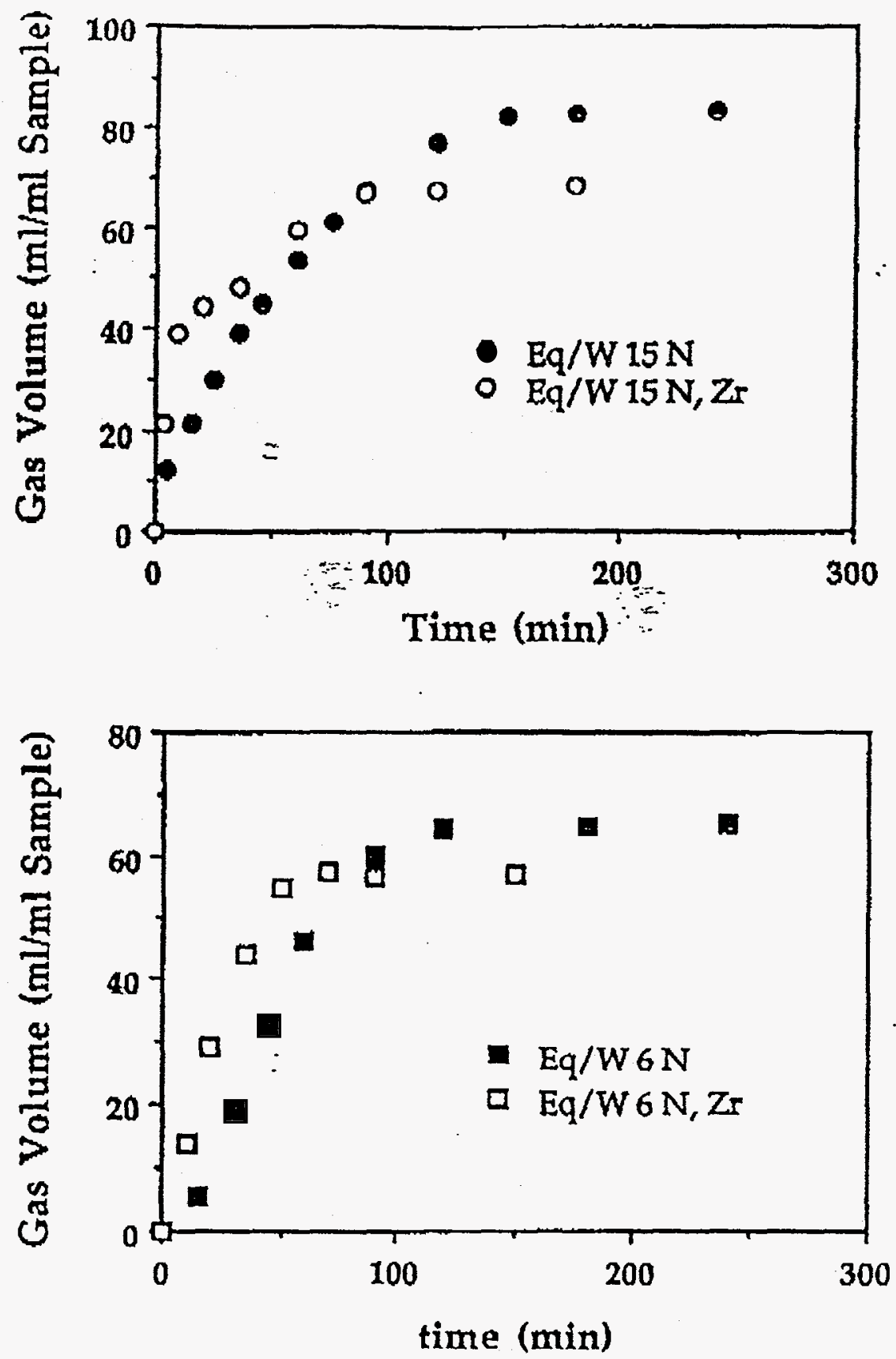

Fig. 4 Effect of Zr4t on Gas production at $150^{\circ} \mathrm{C}$ 


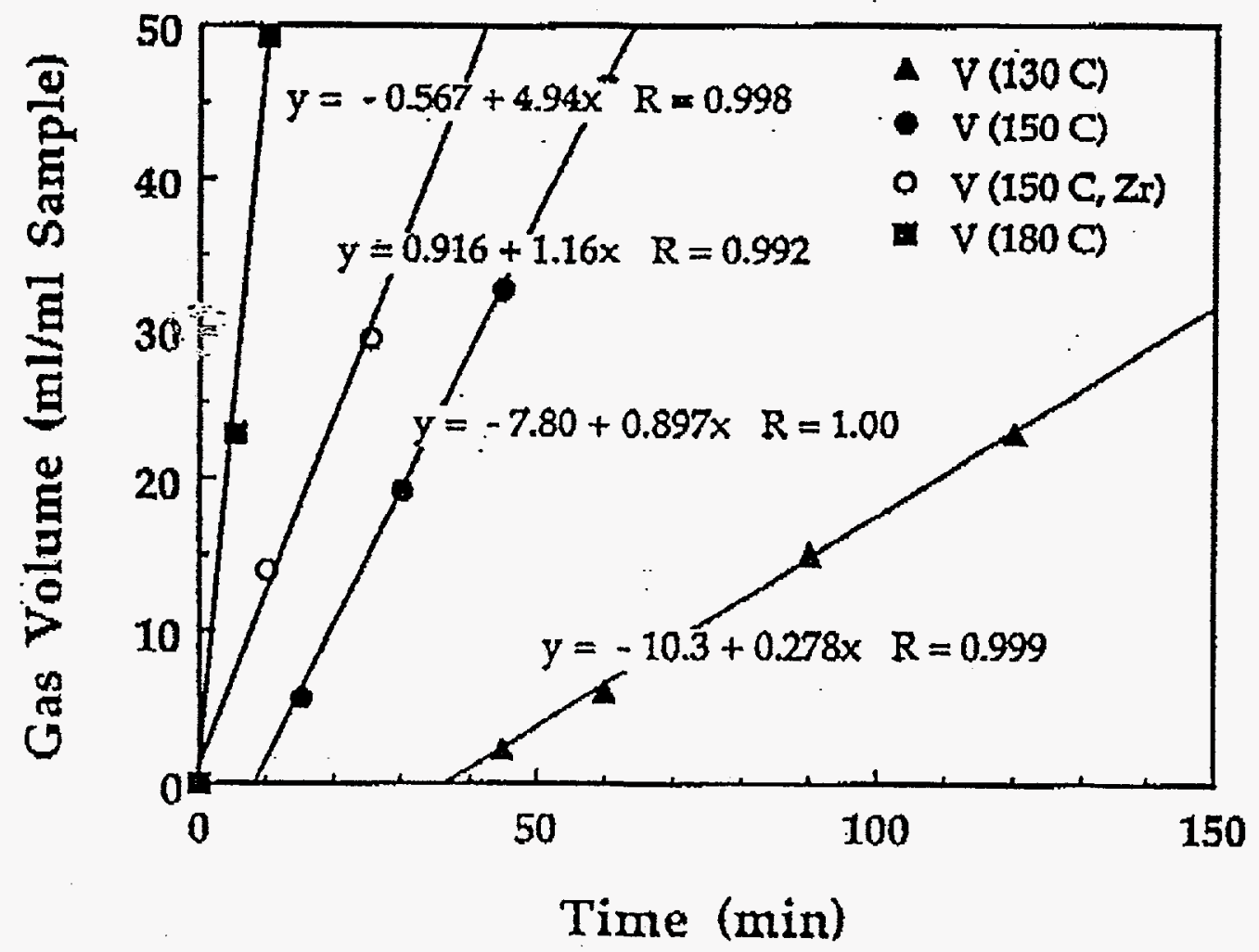

Fig. 5 Effect of temperature and catalyst on the induction period 


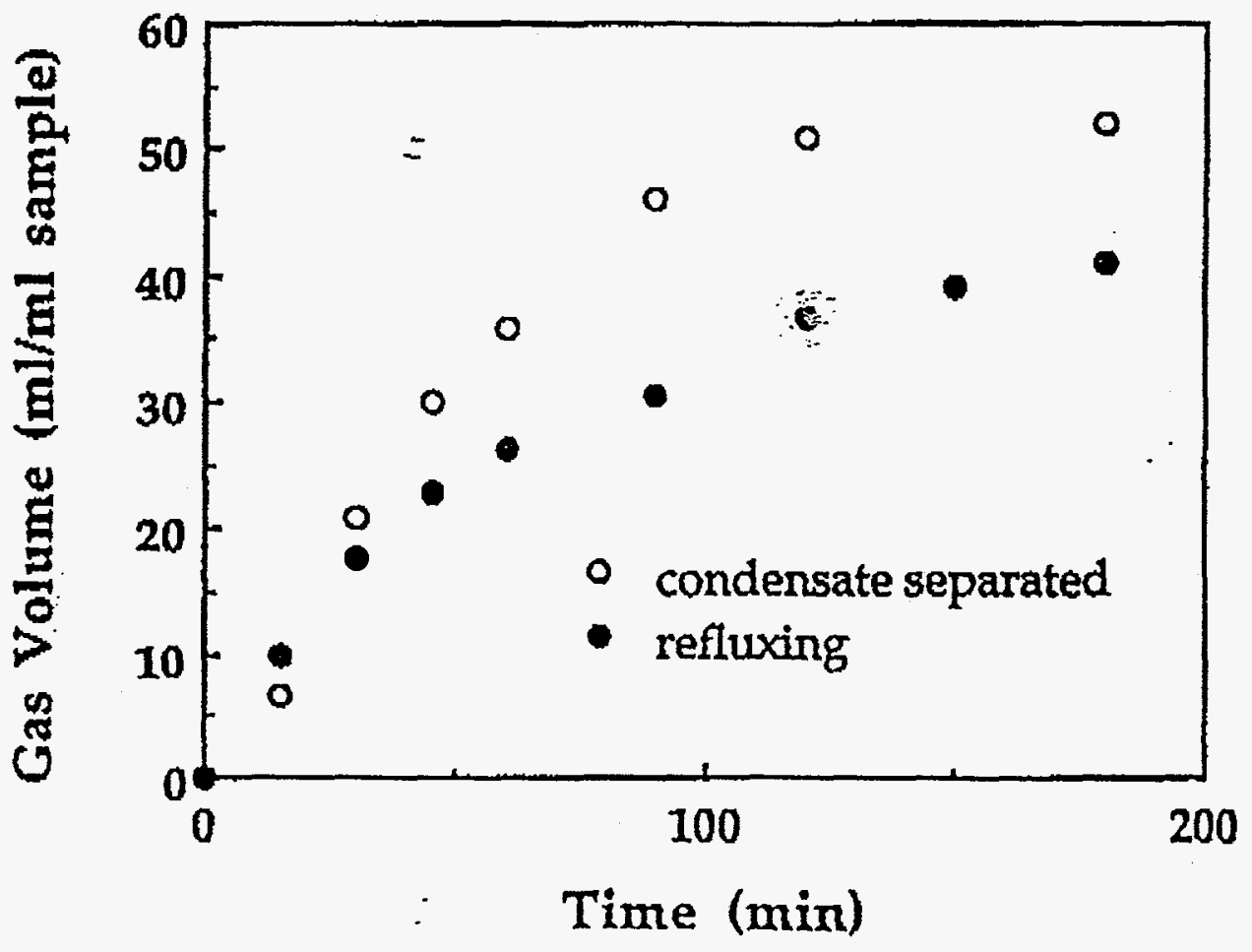

Fig. 6 Effect of product distillation on gas production (150 ${ }^{\circ} \mathrm{C}, \mathrm{TBP}$ eq $/ \mathrm{w} 6 \mathrm{~N}$ HNO3) 


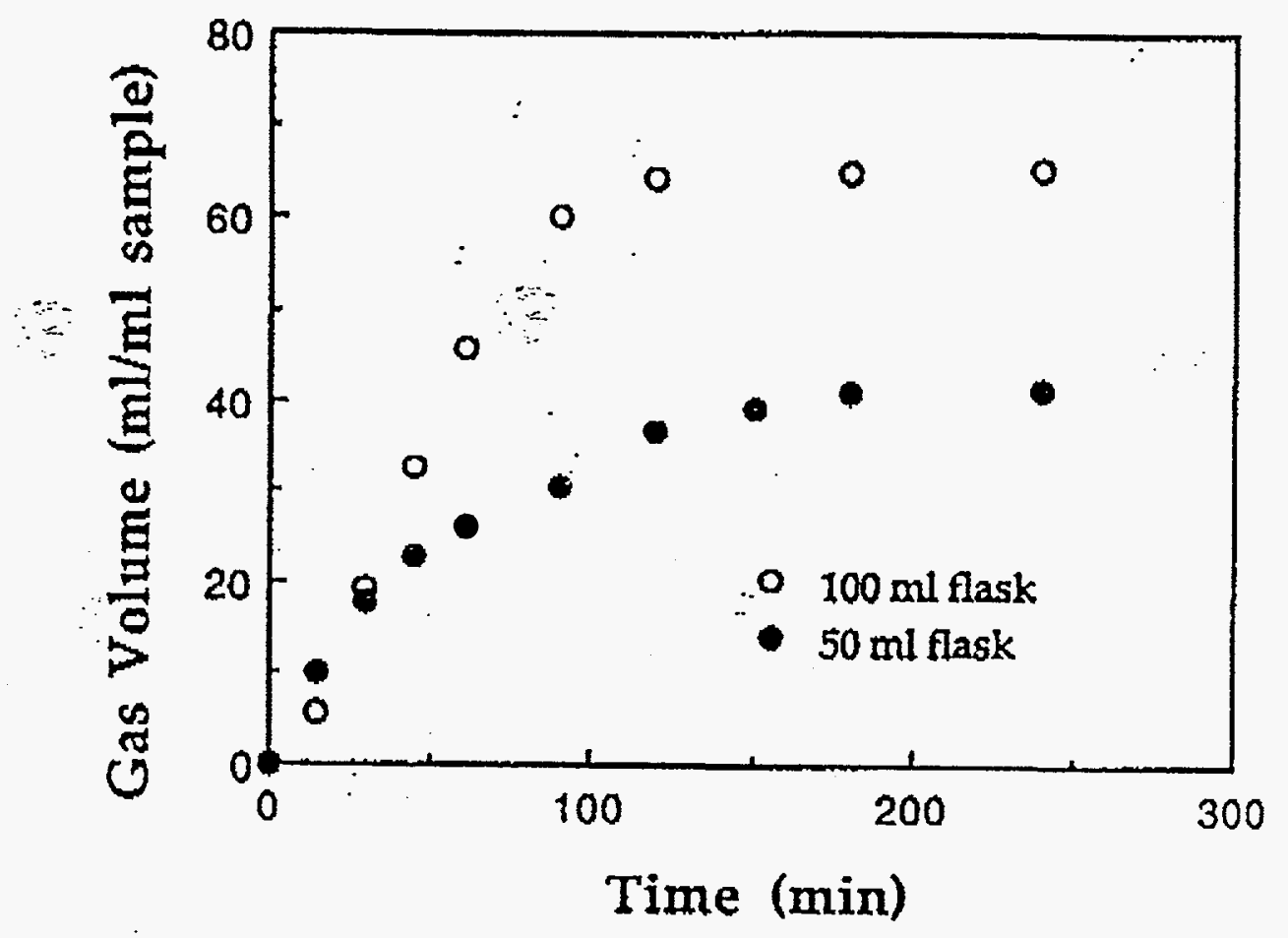

Fig. 7 Effect of Heating Surface on Gas Production $\left(150^{\circ} \mathrm{C}, \mathrm{TBP}\right.$ eq/w $\left.6 \mathrm{~N} \mathrm{HNO} 3\right)$ 


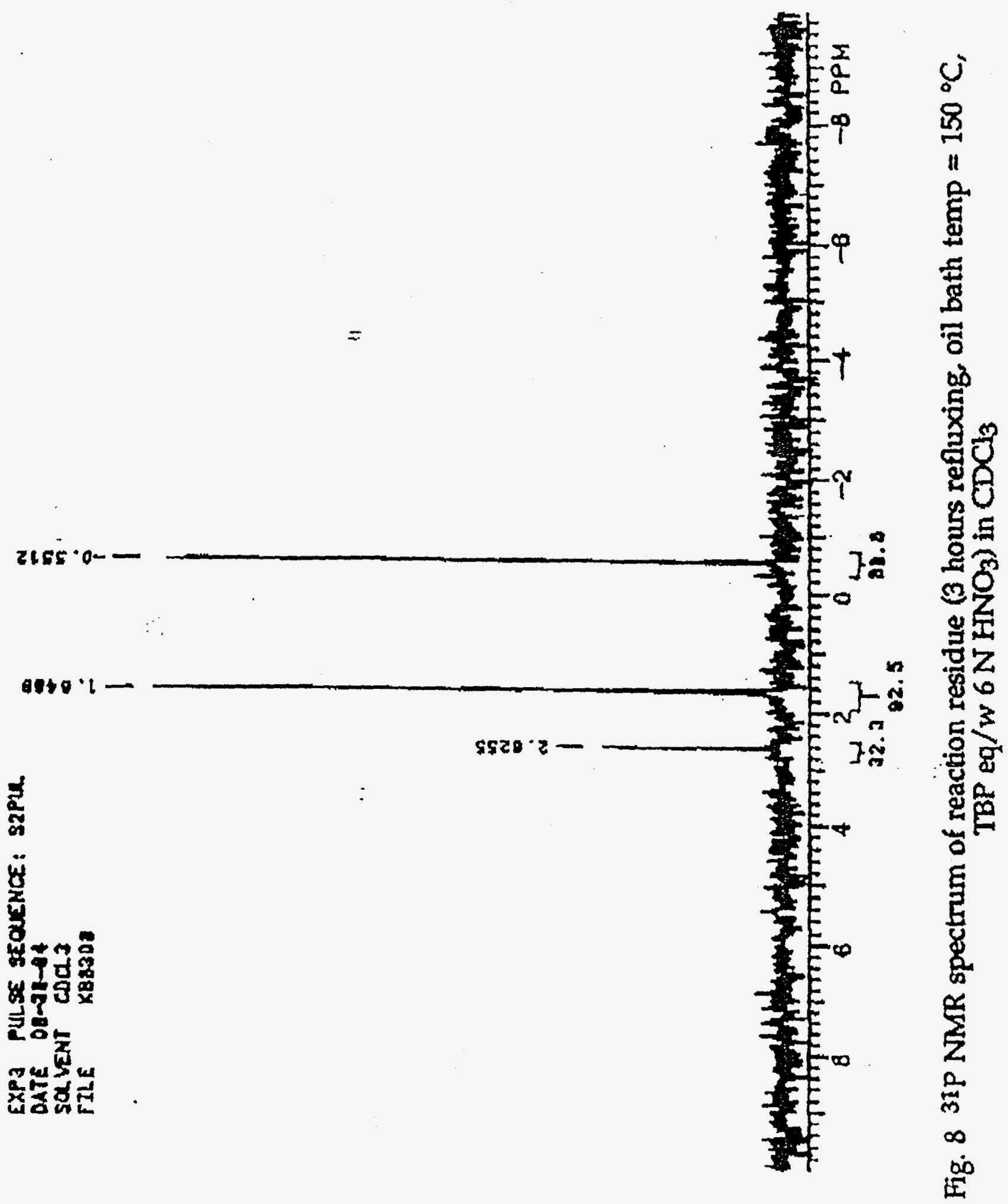




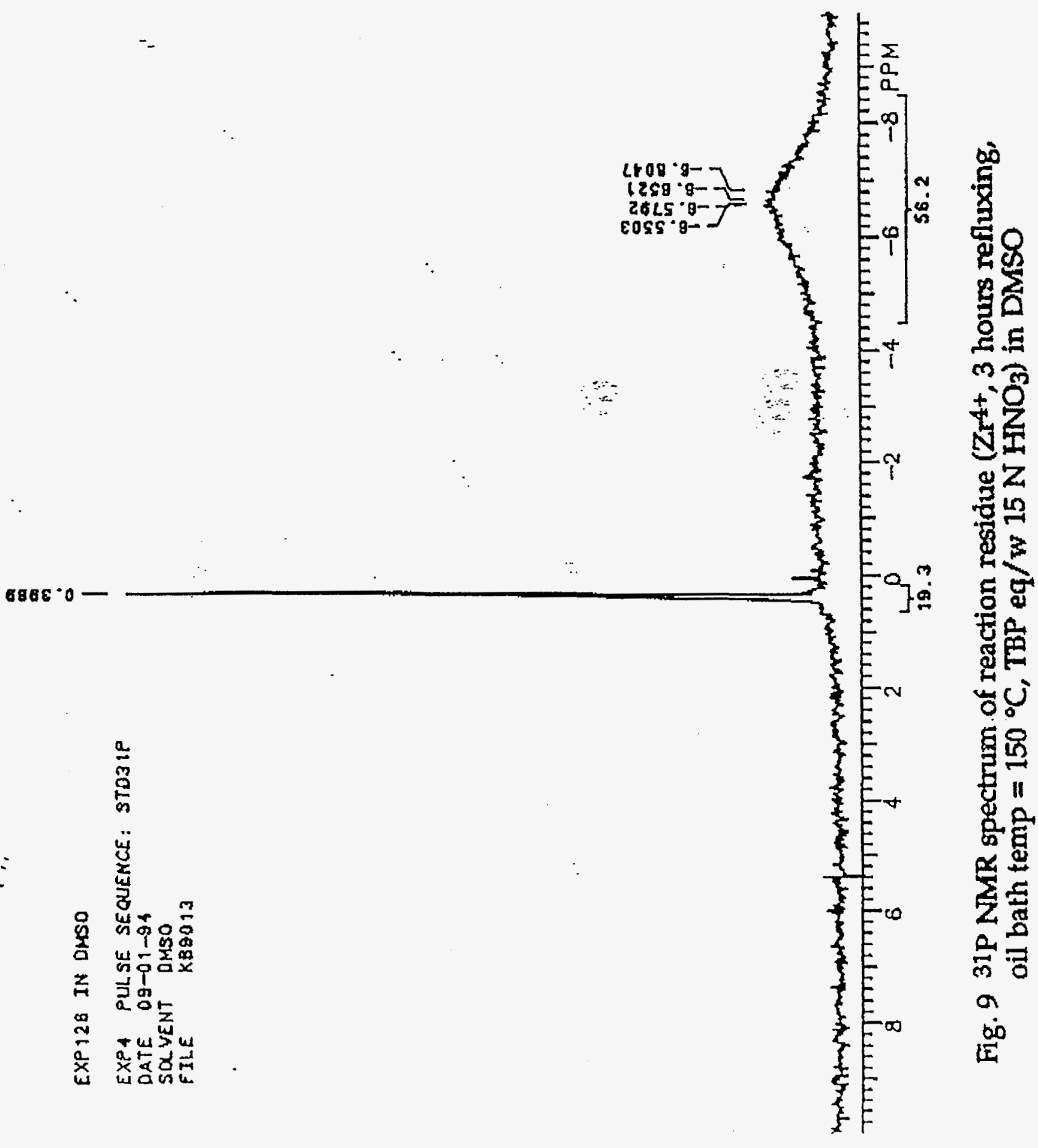




\section{Table 1 \\ Mass Balance}

\section{EXP \#}

No.117 No.119

No.123

No.124 No.125

No.129

No.130

Bath

Temp. $\left({ }^{\circ} \mathrm{C}\right)$

$180 \quad \ldots \quad 180$

180

130

150

150

150

Reaction

Rime (hr)

Equilibrated

Aqueous acid

Conc. (N)

Reaction

Vessel (ml)

1

3

6

6

3

3

$6 \quad 6$

15

15

15

6

6

Reflux or

Distillation

$$
100
$$

100

100

100

100

50

50

and $\%$ as of

Sample Mass

Product Mass (g)

Sample

Mass

Residue

R $\quad \mathrm{R}$

$\mathrm{R}$

R

R

R

R

D

2.20

5.44

5.47

5.10

Gas

5.10

Condensate

$\begin{array}{lllllll}4.32 & 2.55 & 1.61 & 4.77 & 4.61 & 4.70 & 4.19 \\ (85 \%) & (81 \%) & (73 \%) & (87 \%) & (84 \%) & (92 \%) & (82 \%) \\ & & & & & & 0.54 \\ & & & & & & (11 \%) \\ & & & & & & 0.23 \\ & & & & & & (6 \%)\end{array}$




\section{GeorgiaTech}

5. I. dbdel-xhalik

Southem : Vuclear Distinguished Professor

Vuclear Engincering Prognm
THE GEORGE W. WOODRUFF SCHOOL OF MECHANTCAL ENGENEERNG

Geoggia Irustitute of Technology Atlanta, Genrgia 30332-04455

USA

October 10, 1994

Mr. D. T. Paddleford

Safety Technology Department

Westinghouse Savannah River Company

1991 S. Centennial Avenue

Aiken, South Carolina 29803-7657

Dear Don:

Auached is the monthly status report for our ERDA contract on the therral decomposition of nitrated TBP. Work is progressing on schedule.

Please feel free to call me if you have any questions.

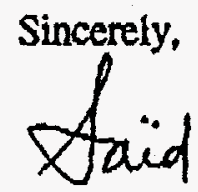

S. I. Abdel-Khalik

\section{SLAK/bc}

cc: Dr. E. K. Barefield

Dr. D. W. Tedder

Dr. R. A. Karam, ERDA

Mr. Pete Dawkins

OCA/Georgia Tech (2) 


\section{Thermal Deconposition of Single Phase TBP/Nitric Acid Mixtures Under Constant Volume Conditions}

During the current project period a new experimental set-up was constructed to measure the pressure changes associated with the thermal decomposition of TBP/nitirc acid mixtures under constant volume conditions. The results for samples containing $3 \mathrm{M}$ and $6 \mathrm{M}$ ritric acid, respectively are given to illustrate the method.

\section{Sample Preparation}

Equilibrated TPB phases containing approximately $3 \mathrm{M}$ and $6 \mathrm{M}$ nitric acid, respectively, were prepared as described in our August 9, 1994 report.

\section{Constant Volume Apparatus}

The apparatus used for constant volume decomposition experiments is shown schematically in Figure 1. The apparatus consists of a $30 \mathrm{~mL}, 316$ stainless steel reaction vessel connected by $0.25^{\prime \prime}$ OD stainless tubing to a pressure sensing device (MI:S Baratron, Model 122A), rupture disk assembly; and separate outlets for connection of a vacuum pump, gas-inlet system and for gas sampling. After loading the cylinder with a sample of known mass, the cylinder is attached to the system with a Swagelok fitting and the entire apparatus is pressurized with two atmospheres of helium and allowed to stand for 20 min to test for leaks. After the leak test the system is evacuated and backfilled with helium ten times to eliminate air from the system. After the last filling with helium the pressure is established at one atmosphere and the system sealed by closing the value to the vacuum outlet. The cylinder is emersed into an oil bath that is preheated to the reaction temperature and pressure readings taken as a function of time until no further pressure increase is observed. At this time the cylinder is removed from the bath and allowed to cool to room temperature where the pressure is again recorded.

\section{Reactant and Product Analyses}

Each equilibrated TBP phase was analyzed prior to reaction for water content by KarlFisher titration, for nitric acid content by titration with $\mathrm{NaOH}$ to a bromthymol blue endpoint, and for TBP concentration by ${ }^{1} \mathrm{H}$ NMR versus $\mathrm{CH}_{2} \mathrm{Cl}_{2}$ as internal standard. A sample of known mass was used in each experiment.

After each reaction was complete, as determined by stabilization of the system pressure, a sample of the gas phase was taken for infrared and mass spectral analysis. These techniques were used for identification of the constitutent gases. Gas chromatography was used for estimation of the gas phase composition. Gas chromatographic analyses were performed using two HewlettPackard Model 5890 chromatographs, each equipped with thermal conductivity detectors, and each connected to a Hewlett-Packard workstation. Samples were taken with a gas-tight syringe and were injected with the syringe into the chromatograph. One of these chromatographs is equipped with a 1/8 $\times 12^{\prime} 5 \mathrm{~A}$ molecular sieves column used for determination of $\mathrm{N}_{2}, \mathrm{O}_{2}, \mathrm{NO}$, and He: argon is used as the carrier gas. The following instrument parameters were used: inlet 
temperature, $120^{\circ} \mathrm{C}$; oven temperature, $32^{\circ} \mathrm{C}$; detector temperature, $120^{\circ} \mathrm{C}$. The second chromatograph, which is equipped with a Porapak $Q$ column, was used for the determination of $\mathrm{CO}, \mathrm{CO}_{2}$ and $\mathrm{N}_{2} \mathrm{O}$. The following instrument parameters were used: inlet temperature, $60^{\circ} \mathrm{C}$; oven temperature, $32^{\circ} \mathrm{C}$; detector temperature, $60^{\circ} \mathrm{C}$. Helium is used as the carrier gas in this instrument.

After completion of all gas sampling, the cylinder was removed from the apparatus and the mass of the residue determined. The composition of the liquid phase was determined by a combination of titrametric and NMR spectroscopic methods. Water and acid content were determined by Karl-Fisher and acid-base titrations, respectively. Butyl nitrate and butyl phosphates were determined by ${ }^{1} \mathrm{H} \mathrm{NMR}$ (versus $\mathrm{CH}_{2} \mathrm{Cl}_{2}$ internal standard). Residual nitric acid will be determined by ${ }^{14} \mathrm{~N}$ NMR but these results are not yet available. The results for equilibrated $\mathrm{TBP} /$ nitric acid phases that contain $3 \mathrm{M}$ and $6 \mathrm{M}$ nitric acid, respectively, are shown in Tables 1 and 2 . 

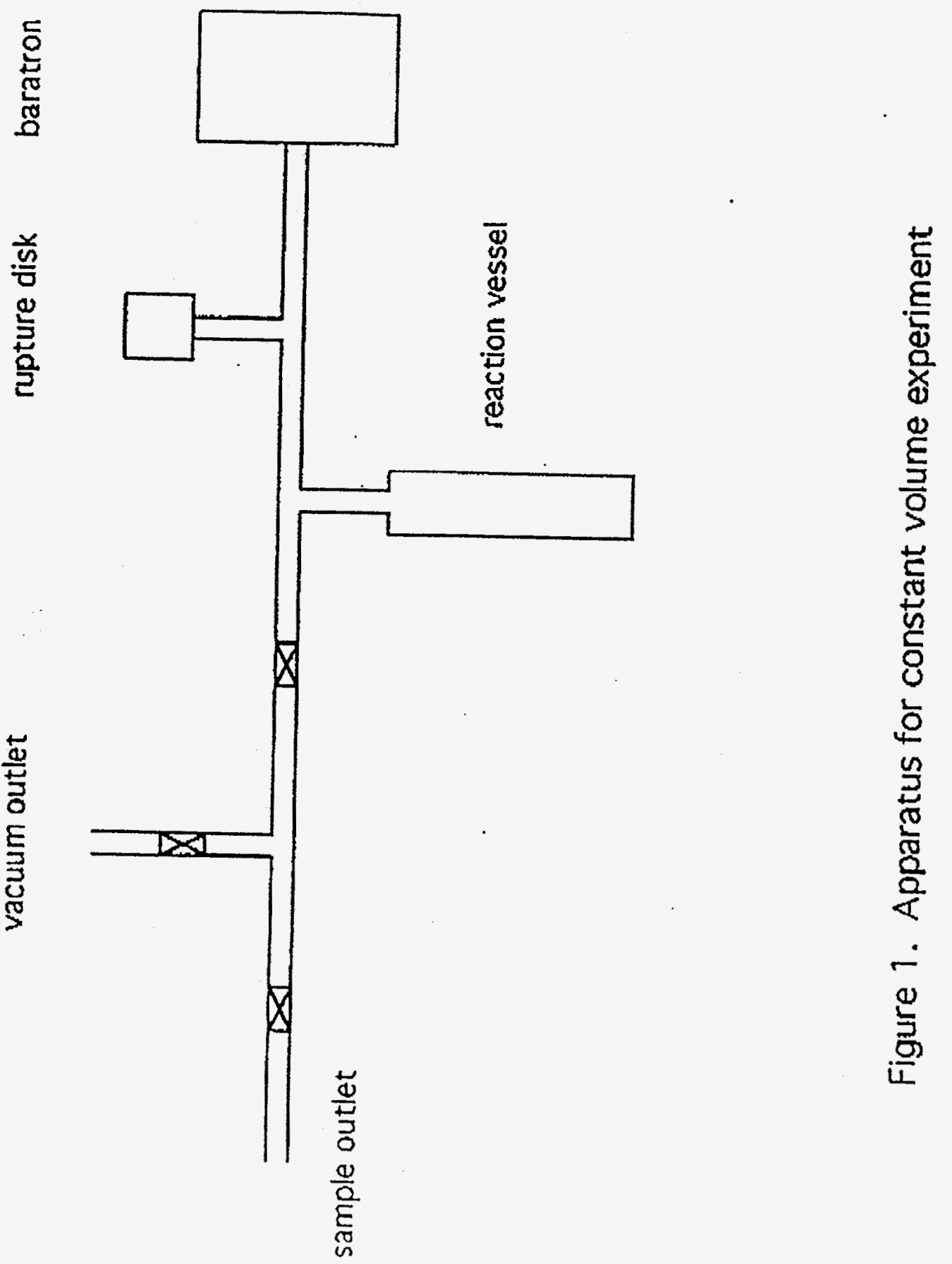


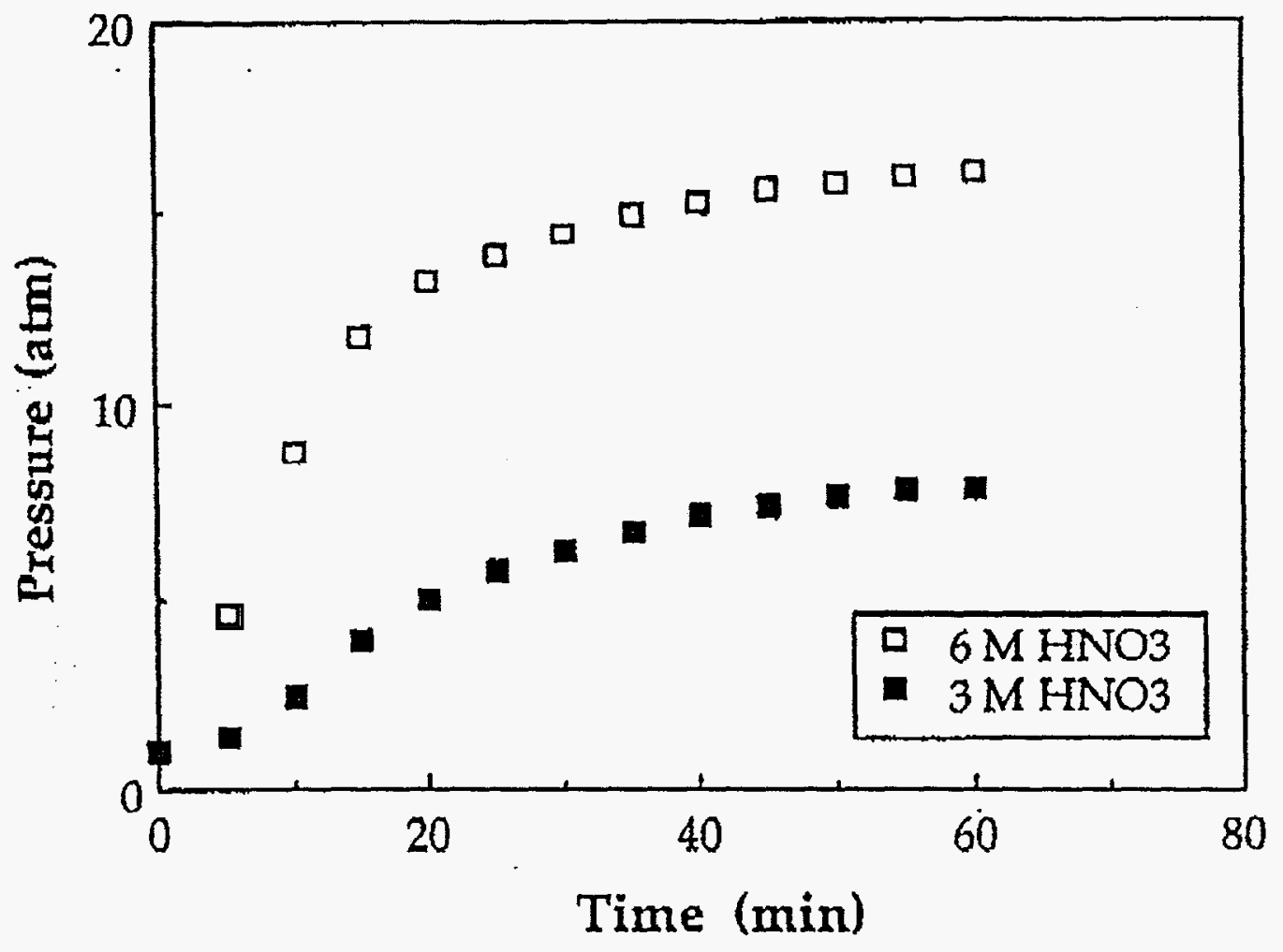

Figure 2. Pressure versus time plots for decomposition of equilibrated TBP-nitric acid phases at $150^{\circ} \mathrm{C}$ 
Table I. Constant Volume Experiment Results (No. 134)

Reaction Temperature: $150^{\circ} \mathrm{C}$

Acid Concentration: $\quad 3 \mathrm{M}$

Sample Volume: $\quad 5 \mathrm{~mL}$

Atmosphere: Helium

Before Reaction:

Sample Weight: $5.10 \mathrm{~g}$

$\left[\mathrm{H}^{+}\right]$:

$15.0 \mathrm{mmö}=(3.00 \mathrm{M})$

$\left[\mathrm{H}_{2} \mathrm{O}\right]:$ - $\quad 7.42 \mathrm{mmol}(1.48 \mathrm{M})$

[TBP]: $\quad 14.16 \mathrm{mmol}(2.83 \mathrm{M})$

After 60 min Reaction:

Sample Weight: $\quad 4.65 \mathrm{~g}$ (weight loss $=0.45 \mathrm{~g}$ )

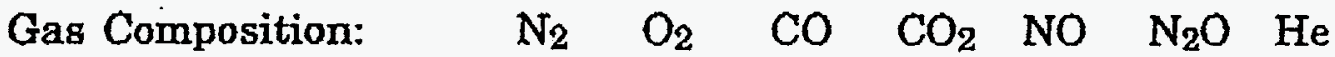

$\begin{array}{llllllll}\text { total mol \% } & 23.7 & 0 & 17.7 & 28.5 & 5.6 & 10.5 & 16.1\end{array}$

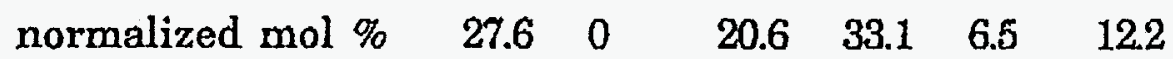

Residue Composition (from acid-base titration, Karl-Fisher titration and $1 \mathrm{H}$ NMR)

$[\mathrm{H}+]$ :

$\left[\mathrm{H}_{2} \mathrm{O}\right]$ :

[TBP]:

[DBP]:

$[\mathrm{BuONO} 2]$ :
$9.43 \mathrm{mmol}(1.89 \mathrm{M})$

$12.9 \mathrm{mmol}(2.58 \mathrm{M})$

$11.13 \mathrm{mmol}(2.23 \mathrm{M})$

$4.77 \mathrm{mmol}(0.95 \mathrm{M})$

$3.00 \mathrm{mmol}(0.60 \mathrm{M})$

\section{Material Balance}

Gas Average Molecular Weight:

31.08

Quantity of Gas Produced:

$13.64 \mathrm{mmol}(-0.42 \mathrm{~g})$

Carbon Element present in Gas:

$6.30 \mathrm{mmol}$

Carbon Element Lost from Aqueous Phase:

$7.08 \mathrm{mmol}$ 


\section{Table II. Constant Volume Experiment Results(No. 135).}

Reaction Temperature: $150^{\circ} \mathrm{C}$

Acid concentration: $\quad 6 \mathrm{M}$

Sample Volume: $\quad 5 \mathrm{~mL}$

Atmosphere: Helium

\section{Before Reaction:}

Sample Weight: $\quad 5.10 \mathrm{~g}$

$[\mathrm{H}+]$ :

$29.25 \mathrm{mmol}(5.85 \mathrm{M})$

$\left[\mathrm{H}_{2} \mathrm{O}\right]: \quad 9.40 \mathrm{mmol}(1.88 \mathrm{M})$

[TBP]: $\quad 13.23 \mathrm{mmol}(2.65 \mathrm{M})$

After 60 min Reaction:

Sample Weight: $\quad 4.25 \mathrm{~g}$ (weight loss $=0.85 \mathrm{~g}$ )

$\begin{array}{llllllll}\text { Gas Composition: } & \mathrm{N}_{2} & \mathrm{O}_{2} & \mathrm{CO} & \mathrm{CO}_{2} & \mathrm{NO} & \mathrm{N}_{2} \mathrm{O} & \mathrm{He}\end{array}$

$\begin{array}{llllllll}\text { total mol \% } & 22.3 & 0 & 18.7 & 32.0 & 9.2 & 10.3 & 16.1\end{array}$

normalized mol \% $24.1 \quad 0 \quad 20.2 \quad 34.6 \quad 9.9 \quad 11.1$

Residue Composition (from acid-base titration, Karl-Fisher titration and 1 H NMR)

$\left[\mathrm{H}^{+}\right]$:

$15.04 \mathrm{mmol}(3.01 \mathrm{M})$

$\left[\mathrm{H}_{2} \mathrm{O}\right]$;

$19.80 \mathrm{mmol}(3.96 \mathrm{M})$

[TBP]:

$7.82 \mathrm{mmol}(1.56 \mathrm{M})$

[DBP]:

$6.92 \mathrm{mmol}(1.38 \mathrm{M})$

[BuONO 2$]:$

$3.51 \mathrm{mmol}(0.70 \mathrm{M})$

Material Balance

Gas Average Molecular Weight:

33.22

Quantity of Gas Produced:

$28.15 \mathrm{mmol}(\sim 0.93 \mathrm{~g})$

Carbon Element present in Gas:

$14.27 \mathrm{mmol}$

Carbon Element Lost from Aqueous Phase:

$13.64 \mathrm{mmol}$ 


\section{GeorgiaTech}

S. 1. Abdel-Ktuallk

Southern Nudear Disstinguished Professor

Nuclear Engineering Program
THE GEORGE W. WOODRUFF SCHOOL OF MECHANICAI ENGLNEERANG

Georgia Institute of Technology

Allanta. Georgia 30332-0405

USA

September 9.1994

Mr. D. T. Paddleford

Safety Technology Deparment

Westinghouse Savannah River Company

1991 S. Centennial Avenue

Aiken, South Carolina 29803-7657

\section{Dear Don:}

Attached is a progress rejiort for our ERDA contract on the thermal decomposition of nitrated TBP. The report deals with our work on single phase experiments during the two-months period of July 11, 1994 through September 9, 1994. Work is progressing on schedule.

Please feel free to call me if you have any questions.

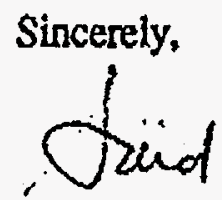

S. 1. Abdel-Khalik

SIAK/bc

cc: Dr. E. K. Barefield

Dr. D. W. Tedder

Dr. R. A. Karam, ERDA

Mr. Pete Dawkins

OCA/GeorgiaTech(2) 


\section{GeorgiaTech}

S. I. Abdel-Khalik

Southern Niclear Distinguished Professor

Nuckear Engincering Program
THE GEORGE W. WOODAUFP SCHOOL OF MECHLANTCAI ENGDVEERING

\section{Georgia lastitute of Techaology} Adanti, Georgia 30332-0405 USA

November 10, 1994

Mr. D. T. Paddleford

Safety Technology Department

Westinghouse Savannah River Company

1991 S. Centeninial Avenue

Aiken, South Carolina 29803-7657

\section{Dear Don:}

Attached is the monthly status report for our ERDA contract on the thermal decomposition of nitrated TBP. We have completed all single phase tests for both coastant volume and constant pressure conditions and are proceeding with similar tests for two-phase systems. Work is progressing on schedule.

Please feel free to call me if you have any questions.

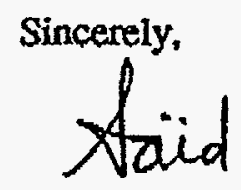

\section{S. I. Abdel-Khalik}

\section{SIAK/bC}

cc: Dr. E. K. Barefield

Dr. D. W. Tedder

Dr. R. A. Karam, ERDA.

Mr. Pete Dawkins

OCA/Georgia Tech (2) 
Send Monthly Status Report by the 10th of each month to:

Dr. Ratib Karam, ERDA

Neely Nuclear Research Center

900 Atlantic Drive

Atlanta, GA 30332-0405

University Georgia Institute of Technology

PI Dr.S. I.Abdel-Khalik

Project Title Thermal Decomposition of Nitrated Tributyl Phosphate

WSRC Technical

Period of Performance: From 10/11/94 To 11/10/94Representative_Don Paddleford

A. Project Accomplishments:

See attached.

B. Milestones achieved (Based on those identffed in the Task Order):

Work is progressing on schedule. As specified in the Task Order, single phase tests for both constant volume and constant pressure have been cornpleted. Similar tests for two-phase systems are in progress.

C. Problems Encountered:

None 


\section{Executive Summary}

Efforts during this period have focussed on completion of a matrix of experiments at constant pressure, further experiments at constant volume, and initiation of experiments on two phase systems. Further consideration of the carbon mass balance for decomposition experiments suggested that additional condensed phase products must be present. Careful reexamination of proton NMR spectra indicates that propionic acid and acetic acid are present in the condensed phase. The single two phase experiment that has been completed indicates a much greater yield of gaseous products, based on TBP, than in either constant pressure or constant volume, single phase decompositions. The composition of the gas phase for the two phase experiment is similar to that of the single phase experiments except that there is less $\mathrm{CO}$ and more nitrogen and carbon dioxide.

\section{Experimental Procedures}

\section{Sample and Standards Preparation}

Preparation of all samples was done by the same procedures outlined in the October 10, 1994 progress report.

\section{Procedure for Constant Pressure and Constant Volume Decompositions}

The procedures for constant pressure and constant volume decompositions were the same as that outlined earlier (October 10,1994 progress report). A preliminary two phase, constant pressure experiment conducted with $5 \mathrm{~mL}$ portions of equilibrated TBP and $5 \mathrm{~mL}$ of aqueous acid produced more gas than the maximum volume of the gas handling apparatus. The experiment described below utilized $2.5 \mathrm{~mL}$ of both TPB and aqueous acid phases, which produced a volume of gas compatible with the apparatus. It is expected that future two-phase experiments will be done using the same protoccol.

\section{Results}

Table 1 lists the experiments completed during the past month. Table 2 sumarizes the results of a single phase, constant pressure experiment that illustrates the current status of our efforts to achieve a carbon mass balance for such experiments. Table 3 outlines the results of the two phase, constant pressure experiment recently completed. Gas evolution versus time plots for these experiments are shown in Figures 1 and 2, respectively. Because these experiments were not performed at the same temperature, they cannot be directly compared. However, it is obvious that the amount of gas produced in the two-phase experiment is much larger, based on TBP, than that produced in the single phase experiment. This must mean that the additional nitrogen gases are produced from nitric acid in the aqueous phase. It is interesting to note that there is no detectable $\mathrm{CO}$ in the two-phase experiment.

Two additional condensed phase products, propionic and acetic acids, have been identified based on the presence of their resonances in the proton NMR spectrum of residues. These resonances were present in the spectra of earlier samples but had not been previously identified. It is curious that butyric acid does not appear in appreciable amounts, and aiso that formic acid is not 
present. Assuming that butyl nitrate is the initial product from reaction of TBP with nitric acid, a logical sequence for formation of carbon fragments could be butyl nitrate - butraldehyde - butyric acid -propionic acid $+\mathrm{CO}_{2} \rightarrow$ acetic acid $+\mathrm{CO}_{2} \neg$ formic acid. Presumably formic acid would finally be oxidizedto carbon dioxide. Butenes have not been identified in either the gaseous or condensed phases. 
Table 1. List of Completed Single Phase Experiments

\section{Constant Pressure}

Exp. No Bath Temp Acid in.TBP Refluxing/Distillation Catalyst

$\begin{array}{lllll}1 & 130^{\circ} \mathrm{C} & 6 \mathrm{M} & \mathrm{D} & - \\ 2 & 150^{\circ} \mathrm{C} & 6 \mathrm{M} & \mathrm{D} & - \\ 3 & 180^{\circ} \mathrm{C} & 6 \mathrm{M} & \mathrm{D} & - \\ 4 & 150^{\circ} \mathrm{C} & 3 \mathrm{M} & \mathrm{D} & - \\ 5 & 150^{\circ} \mathrm{C} & 6 \mathrm{M} & \mathrm{D} & \mathrm{Zr} \\ 6 & 130^{\circ} \mathrm{C} & 6 \mathrm{M} & \mathrm{R} & - \\ 7 & 150^{\circ} \mathrm{C} & 6 \mathrm{M} & \mathrm{R} & - \\ 8 & 180^{\circ} \mathrm{C} & 6 \mathrm{M} & \mathrm{R} & -\end{array}$

II. Constant Volume

$\begin{array}{cccc}\text { Exp. No } & \text { Bath Temp } & \text { Acid in TBP } & \text { Catalyst } \\ 1 & 130^{\circ} \mathrm{C} & 6 \mathrm{M} & - \\ 2 & 150^{\circ} \mathrm{C} & 6 \mathrm{M} & - \\ 3 & 180^{\circ} \mathrm{C} & 6 \mathrm{M} & - \\ 4 & 150^{\circ} \mathrm{C} & 3 \mathrm{M} & - \\ 5 & 150^{\circ} \mathrm{C} & 6 \mathrm{M} & \mathrm{Zr} \mathrm{r}^{4+}\end{array}$


Table 2. Results for Typical Single Phase Experiment at Constant Pressure

Bath Temperature: $\quad 130^{\circ} \mathrm{C}$

Acid concentration: $\quad 6 \mathrm{M}$

Sample Volume: $\quad 5 \mathrm{ml}$..

Atmosphere: Helium

Distillation/Refluxing $\mathbf{R}$

Before Reaction:

Sample Weight: $\quad 5.41 \mathrm{~g}$

[H+]:

$28.75 \mathrm{mmol}(5.75 \mathrm{M})$

$\left[\mathrm{H}_{2} \mathrm{O}\right]: \quad 9.40 \mathrm{mmol}(1.88 \mathrm{M})$

[TBP]: $\quad 13.30 \mathrm{mmol}(2.66 \mathrm{M})$

\section{After Reaction:}

Sample Weight: $\quad 4.74 \mathrm{~g}$ (weight loss $=0.67 \mathrm{~g}$ )

Total Gas Volume: $\quad 350 \mathrm{ml}$

Gas Composition: $\quad \mathrm{N}_{2} \quad \mathrm{O}_{2} \quad \mathrm{CO} \quad \mathrm{CO}_{2} \quad \mathrm{NO} \quad \mathrm{N}_{2} \mathrm{O} \quad \mathrm{He}$

$\begin{array}{llllllll}\text { total mol \% } & 1.4 & 0 & 6.7 & 19.4 & 50.2 & 3.9 & 15.9\end{array}$

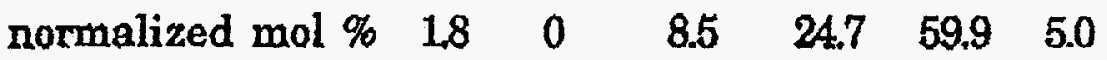

Residue Composition (from acid-base titration, Karl-Fisher titration and ${ }^{1} \mathrm{H}$ NMR)

[H+]:

$20.05 \mathrm{mmol}(4.01 \mathrm{M})$

[ $\mathrm{H}_{2} \mathrm{O}$ ]:

$7.10 \mathrm{mmol}(1.42 \mathrm{M})$

[TBP]**:

$8.30 \mathrm{mmol}(1.66 \mathrm{M})$

[DBP] $]^{* *}$

$5.00 \mathrm{mmol}(1.00 \mathrm{M})$

$\left[\mathrm{BuONO}_{2}\right]$ :

$2.42 \mathrm{mmol}(0.48 \mathrm{M})$

[CH $\left.\mathrm{CH}_{3} \mathrm{CH}_{2} \mathrm{COOH}\right] \quad 0.61$ monol $(0.12 \mathrm{M})$

$\left[\mathrm{CH}_{3} \mathrm{COOH}\right] \quad 0.61 \mathrm{mmol}(0.12 \mathrm{M})$

\section{Material Balaace}

Carbon element loss from residue:

$7.27 \mathrm{mmol}$

Carbon element present in gas Phase:

$4.78 \mathrm{mmol}$

** SIP NMR spectra show no loss in phosphor species so the sum of TBP and DBP concentrations after reaction equals to TBP concentration before reaction 
Table 3. Results of Typical Two Phases Experiment at Constant Pressure

Bath Temperature:

$150^{\circ} \mathrm{C}$

Acid concentration: $\quad 6 \mathrm{M}$

Sample Volume:

$2.5 \mathrm{ml}$ each phase

Atmosphere: Helium

Distillation/Refluxing $\mathbf{R}$

\section{Before Reaction:}

Sample Weight: $\quad 2.65 \mathrm{~g}$ of organic and $3.51 \mathrm{~g}$ of aqueous phase

$\left[\mathrm{H}^{+}\right]$:

$\left[\mathrm{H}_{2} \mathrm{O}\right]:$

[TBP]:
$14.5 \mathrm{mmol}$ (5.80 M, organic) and $36.13 \mathrm{mmol}$ (14.45 M, aqueous)

$4.70 \mathrm{mmol}$ (1.88 $\mathrm{M}$, organic)

$6.65 \mathrm{mmol}(2.66 \mathrm{M})$

\section{After Reaction:}

Sample Weight: $1.91 \mathrm{~g}$ of organic (loss $=0.74 \mathrm{~g}$ ) and $3.05 \mathrm{~g}$ of aqueous (loss $=0.56 \mathrm{~g}$ ) Total Gas Volume: $\quad 775 \mathrm{ml}$

Gas Compogition: $\quad \mathrm{N}_{2} \quad \mathrm{O}_{2} \quad \mathrm{CO} \quad \mathrm{CO}_{2} \quad \mathrm{NO} \quad \mathrm{N}_{2} \mathrm{O} \quad \mathrm{He}$

$\begin{array}{llllllll}\text { total mol \% } & 6.9 & 0 & 0 & 24.2 & 40.1 & 6.4 & 20.7 \\ \text { normalized mol \% } & 8.9 & 0 & 0 & 31.2 & 51.7 & 8.2 & \end{array}$

Residue Composition (from acid-base titration, Karl-Fisher titration and ${ }^{1} \mathrm{H}$ NMR) $\left[\mathrm{H}^{+}\right]$: $23.1 \mathrm{mmol}(9.24 \mathrm{M}$, aqueous) and $13.75 \mathrm{mmol}(5.50 \mathrm{M}$, organic)

$\left[\mathrm{H}_{2} \mathrm{O}\right]$ : $8.18 \mathrm{mmol}$ (3.27 $\mathrm{M}$, organic)

[TBP]**: $3.40 \mathrm{mmol}(1.36 \mathrm{M})$

$[D B P]^{* *}$ :

$3.25 \mathrm{mmol}(1.30 \mathrm{M})$

$\left[\mathrm{BuONO}_{2}\right]:$

$0.17 \mathrm{mmol}(0.07 \mathrm{M})$

$\left[\mathrm{CH}_{3} \mathrm{CH}_{2} \mathrm{COOH}\right] \quad 0.35 \mathrm{mmol}(0.14 \mathrm{M})$

$\left[\mathrm{CH}_{3} \mathrm{COOH}\right] \quad 0.19 \mathrm{mmol}(0.08 \mathrm{M})$

\section{Material Balance}

Carbon element loss from residue: $10.89 \mathrm{mmol}$

Carbon element present in gas Phase: $9.59 \mathrm{mmol}$

** 31p NMR spectra show no loss in phosphor species so the sum of TBP and DBP concentrations after reaction equals to TBP concentration before reaction 


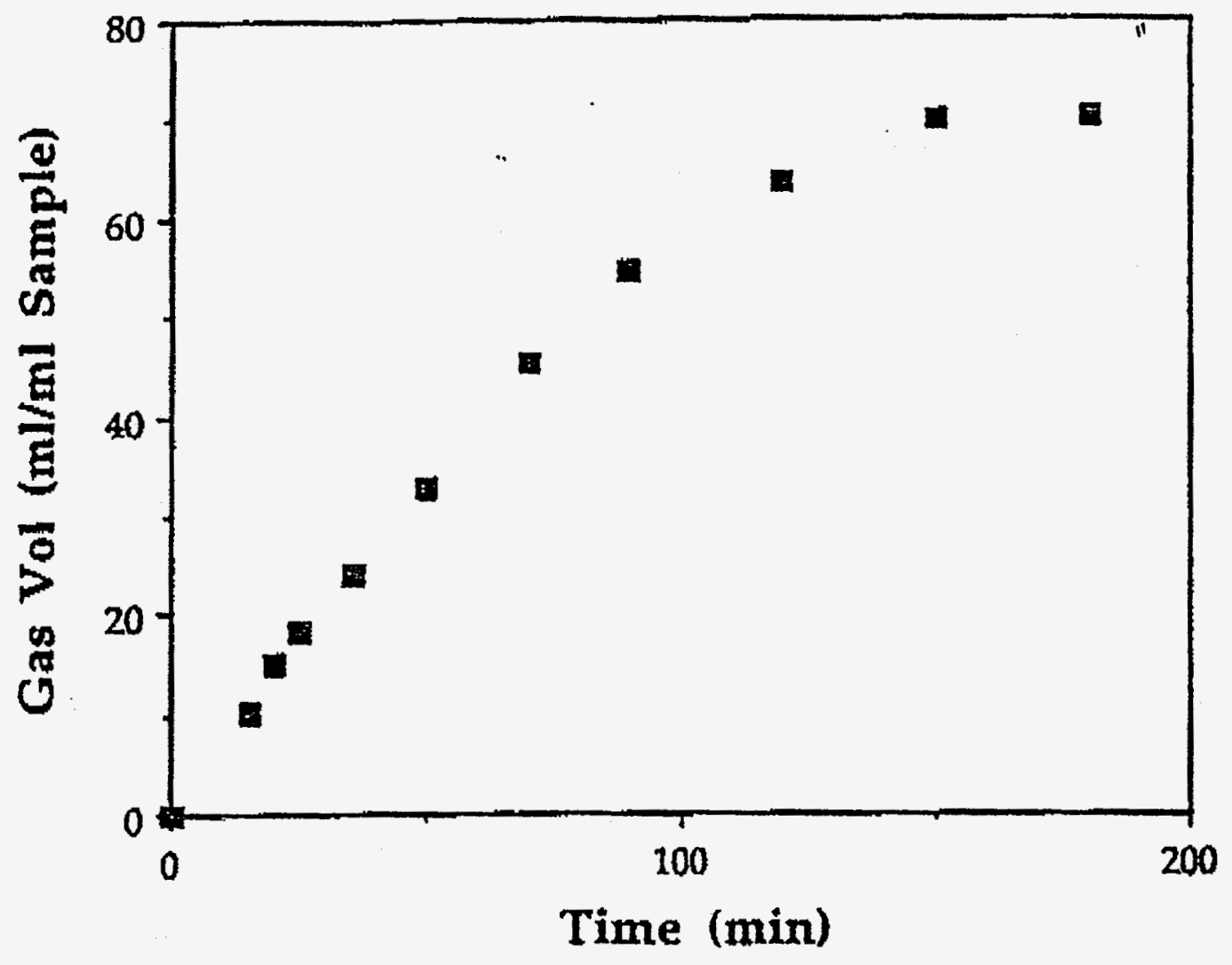

Figure 1. Gas Volume vs Time Plot for Single Phase Hatment $\left(130^{\circ} \mathrm{C}, 6 \mathrm{M} \mathrm{HNO}_{3}\right.$ in TBP, refluring) 


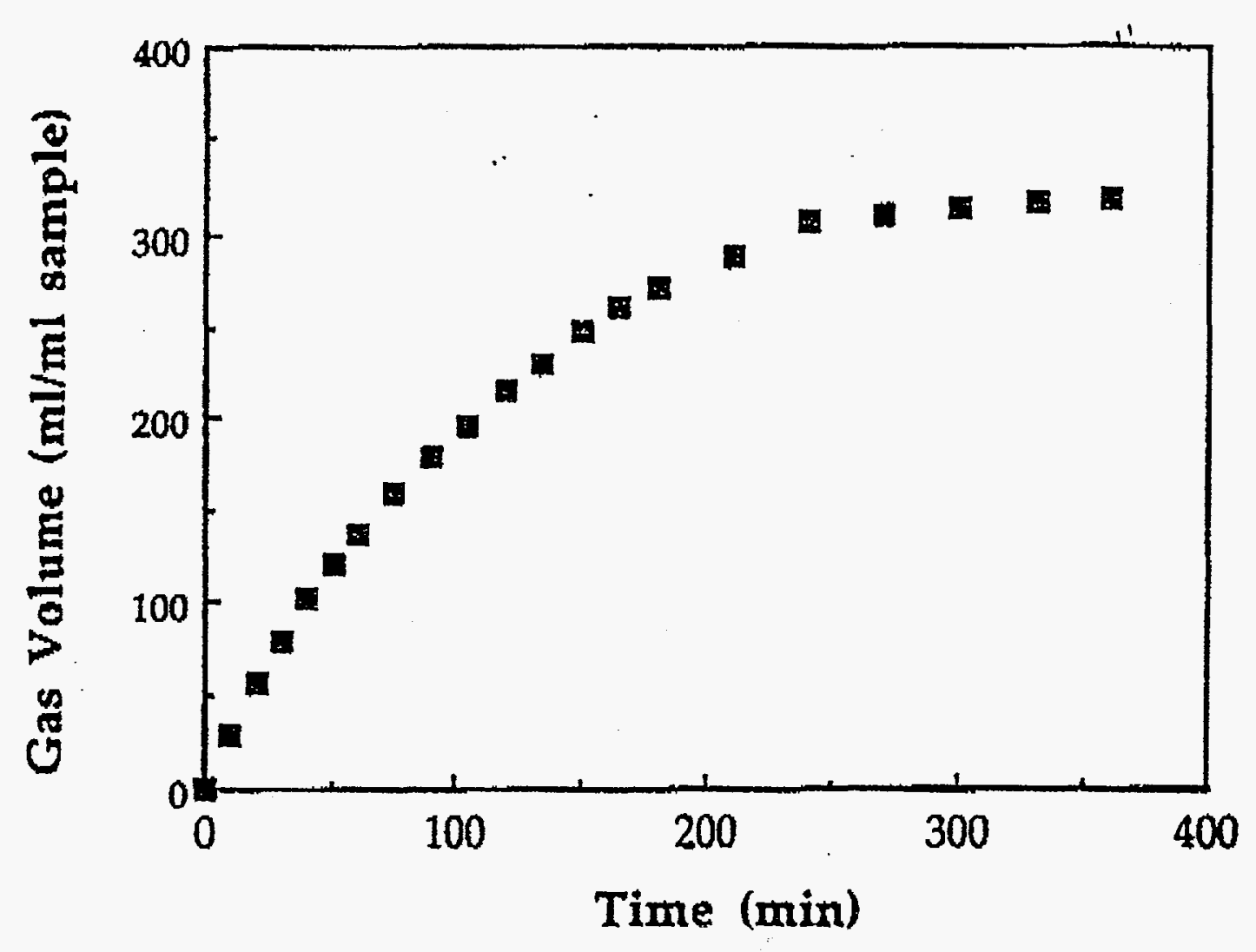

Figure 2. Gas Volume vs Time Plot for Two Phace Experdamat (160 ${ }^{\circ} \mathrm{C}, 2.5 \mathrm{ml}$ of each phase, $6 \mathrm{M}$ BNO $\mathrm{g}$ in TBP, refluxing) 


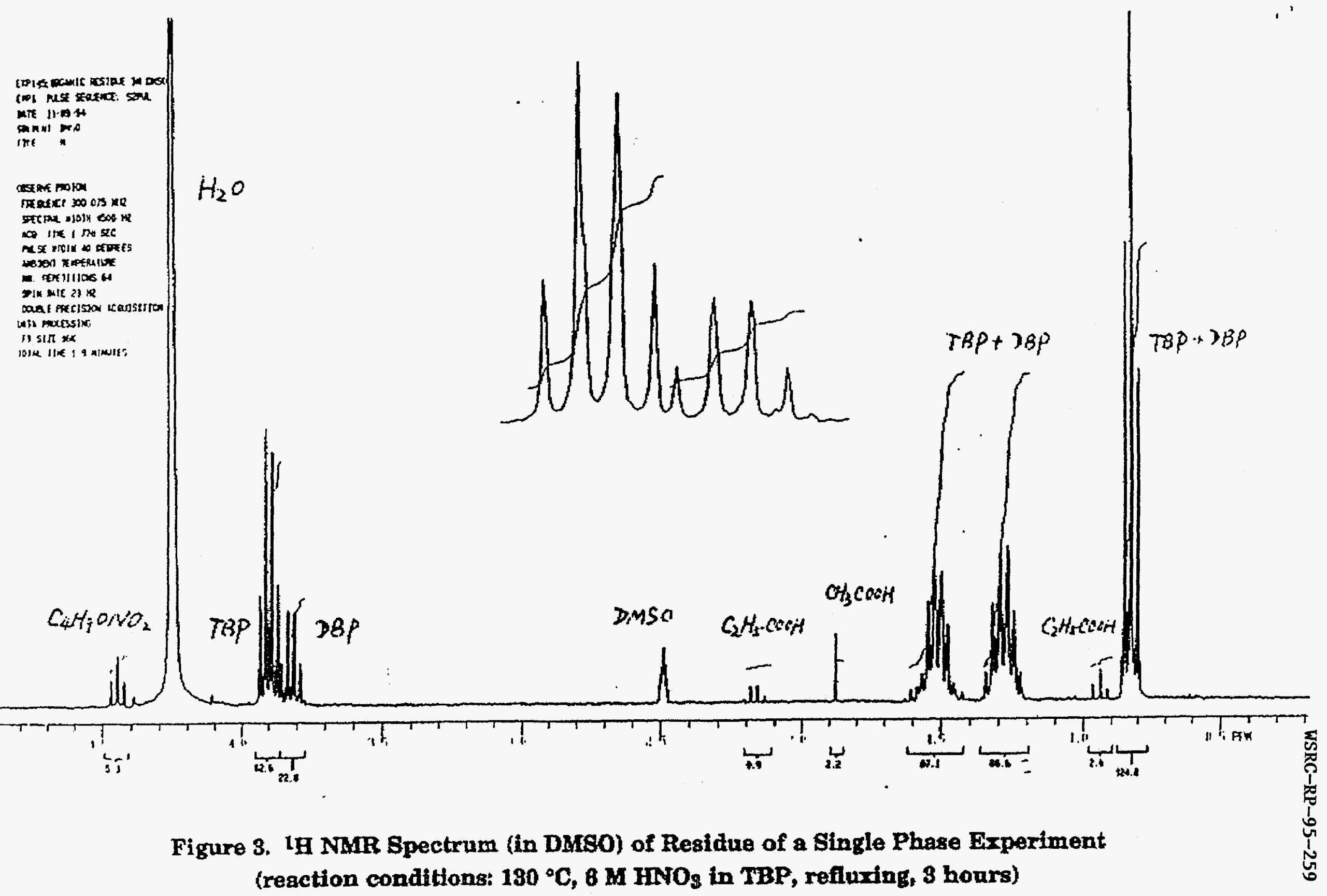




\section{GeorgiaTech}

S. L. Abdelkthalik

Southem Nucte2t Distinguished Professor

Nuclear Engincering Progam
THE GRORGE W. WOODRUFE SCHOOT OF MECHANCAI ENGINERRTNO

Georgia Institute of Technology Allanis. Ceorgta 30332-0105

IISA

January 9,1995

Mr. D. T. Paddleford

Safety Technology Department

Westinghouse Savannah River Company

1991 S. Centennial Avenue

Aiken. South Carolina 29803-7657

Dear Don:

Artached is the monthly status report for our ERDA contract on the thermal decomposition of nitrated TBP. We have completed all experiments and are in the process of analyzing the data and preparing the final report.

The final report will be transmitsed to you on January 31,1995 . We look forward to seeing you in early February.

Please feel free to call me if you have any questions.

Sincerely,

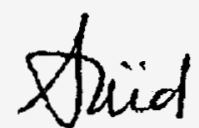

S. I. Abdel-Khalik

SIAK/bc

cc: Dr. E. K. Barefield

D. D. W. Tedder

Dr. R. A. Karam, ERDA

Mr. Pete Dawkins

OCAVGeorgia Tech (2) 
Send Monthly Status Report by the 10th of each month to:

Dr. Ratib Karam, ERDA

Neely Nuclear Research Center

900 Atiantic Drive

Atlanta, GA 30332-0405

University Georgia Institute of Technology

PI Dr.S. I. Abdel-Khalik

Project TItle Thermal Decomposition of Nitrated Tributyl Phosphate

WSRC Technical

Perlod of Performance: From 12/11/94To 1/10/95Representative Don Paddleford

A. Project Accomplishments:

All experiments have been completed.

B. Milestones achleved (Based on those Identffed in the Task Order):

Work is progressing on schedule. As specified in the Task Order, all single phase and two-phase experiments have been completed (see attached table). Work is underway to analyze the data and prepare the final report.

C. Problems Encountered:

None 
TABLE 1

\section{LIST OF COMPLETED EXPERIMENTS}

\section{Single Phase}

\section{Constant Pressure}

Exp. No Bath Temp Acid in TBP Refluxing/Distillation Catalyst

$\begin{array}{lllll}136 & 150^{\circ} \mathrm{C} & 3 \mathrm{M} & \mathrm{D} & - \\ 137 & 150^{\circ} \mathrm{C} & 6 \mathrm{M} & \mathrm{D} & - \\ 139 & 130^{\circ} \mathrm{C} & 6 \mathrm{M} & \mathrm{D} & - \\ 140 & 150^{\circ} \mathrm{C} & 6 \mathrm{M} & \mathrm{D} & \mathrm{Zr}{ }^{4+} \\ 141 & 180^{\circ} \mathrm{C} & 6 \mathrm{M} & \mathrm{D} & - \\ 144 & 150^{\circ} \mathrm{C} & 6 \mathrm{M} & \mathrm{R} & - \\ 145 & 130^{\circ} \mathrm{C} & 6 \mathrm{M} & \mathrm{R} & - \\ 146 & 180^{\circ} \mathrm{C} & 6 \mathrm{M} & \mathrm{R} & -\end{array}$

2. Constant Volume

\begin{tabular}{cccc} 
Exp. No & Bath Temp & Acid in TBP & Catalyst \\
\cline { 2 - 3 } 134 & $150^{\circ} \mathrm{C}$ & $3 \mathrm{M}$ & - \\
135 & $150^{\circ} \mathrm{C}$ & $6 \mathrm{M}$ & - \\
142 & $130^{\circ} \mathrm{C}$ & $6 \mathrm{M}$ & - \\
143 & $180^{\circ} \mathrm{C}$ & $6 \mathrm{M}$ & - \\
163 & $150^{\circ} \mathrm{C}$ & $6 \mathrm{M}$ & $\mathrm{Zr}^{4+}$
\end{tabular}

\section{Two Phase}

\section{Constant Pressure}

Exp. No Bath Temp Acid in TBP Refluxing/Distillation Catalyst

$\begin{array}{lll}150 & 150^{\circ} \mathrm{C} & 6 \mathrm{M} \\ 151 & 150^{\circ} \mathrm{C} & 3 \mathrm{M} \\ 152 & 150^{\circ} \mathrm{C} & 6 \mathrm{M} \\ 153 & 180^{\circ} \mathrm{C} & 6 \mathrm{M} \\ 154 & 130^{\circ} \mathrm{C} & 6 \mathrm{M} \\ 159 & 180^{\circ} \mathrm{C} & 6 \mathrm{M} \\ 160 & 130^{\circ} \mathrm{C} & 6 \mathrm{M} \\ 161 & 150^{\circ} \mathrm{C} & 6 \mathrm{M}\end{array}$

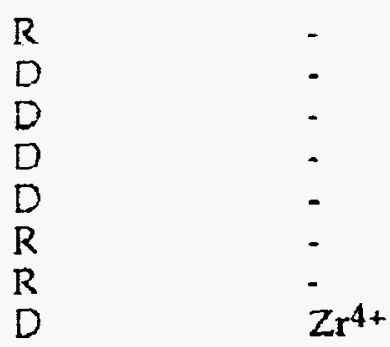




\begin{tabular}{cccc}
2. & \multicolumn{2}{c}{ Constant Volume } \\
Exp. No & Bath Temp & Acid in TBP & Catalyst \\
& nath & & \\
155 & $150^{\circ} \mathrm{C}$ & $6 \mathrm{M}$ & - \\
156 & $150^{\circ} \mathrm{C}$ & $3 \mathrm{M}$ & - \\
157 & $130^{\circ} \mathrm{C}$ & $6 \mathrm{M}$ & - \\
158 & $180^{\circ} \mathrm{C}$ & $6 \mathrm{M}$ & - \\
162 & $150^{\circ} \mathrm{C}$ & $6 \mathrm{M}$ & $\mathrm{Zr}+$
\end{tabular}

III. Additional Experiments

1. Single Phase with No Water TBP (Purged Samples)

Exp. No Bath Temp Acid in TBP Const. Pressure/Volume Catalyst

$\begin{array}{llll}147 \mathrm{~A} & 150^{\circ} \mathrm{C} & -3 \mathrm{M} & \text { Const. Pressure (D) } \\ 147 \mathrm{~B} & 150^{\circ} \mathrm{C} & -3 \mathrm{M} & \text { Const. Volume }\end{array}$

2. Reproducibility Experiments

Exp. No Checking Exp. No

$148 \mathrm{~A} \quad 137$

$148 \mathrm{~B} \quad 136$

$148 \mathrm{C} \quad 140$ 